\title{
Synthesis of gem-Difluorinated Fused Quinolines viaVisible Light-mediated Cascade Radical Cyclization
}

Tiebo Xiao, Linyong Li, Yang Xie, Zong-wan Mao and Lei Zhou*

School of Chemistry and Chemical Engineering, Sun Yat-Sen University, 135 Xingang

West Road, Guangzhou 510275, China

E-mail:zhoul39@mail.sysu.edu.cn

\section{Supporting Information}

\section{Table of Contents}

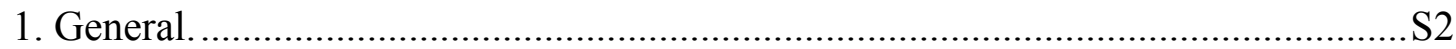

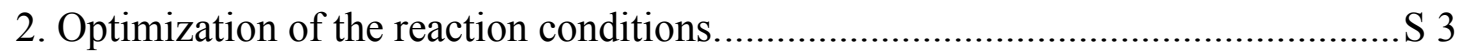

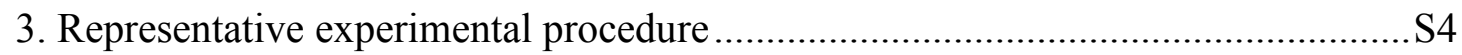

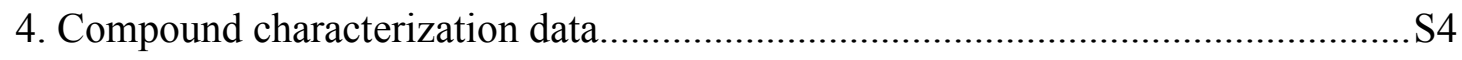

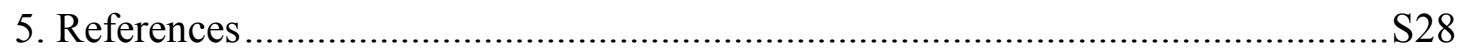

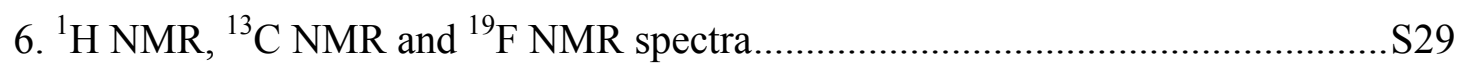


General Unless otherwise noted, all reactions were performed in a $10 \mathrm{~mL}$ test tube at room temperature. Photo-irradiation was carried out with a $5 \mathrm{~W}$ blue LED. For chromatography, 200-300 mesh silica gel (Qingdao, China) was employed. ${ }^{1} \mathrm{H}$ NMR and ${ }^{13} \mathrm{C}$ NMR spectra were measured in $\mathrm{CDCl}_{3}$ and recorded on Brucker ARX 400 spectrometer. Chemical shifts $(\delta)$ were given in ppm, referenced to the residual proton resonance of $\mathrm{CDCl}_{3}$ (7.26), to the carbon resonance of $\mathrm{CDCl}_{3}$ (77.16). Coupling constants $(\mathrm{J})$ were given in Hertz $(\mathrm{Hz})$. The term $\mathrm{m}, \mathrm{q}, \mathrm{t}, \mathrm{d}, \mathrm{s}$ referred to multiplet, quartet, triplet, doublet, singlet. Exact masses (HRMS) were recorded on a high resolution magnetic mass spectrometer using electron impact ionization techniques. Substrates 5a-s and $\mathbf{1 4}$ were prepared according to previous reported procedures. ${ }^{[1]}$ Materials obtained from commercial suppliers were used without further purification. 
Table S1. Optimization of the reaction conditions. ${ }^{a}$

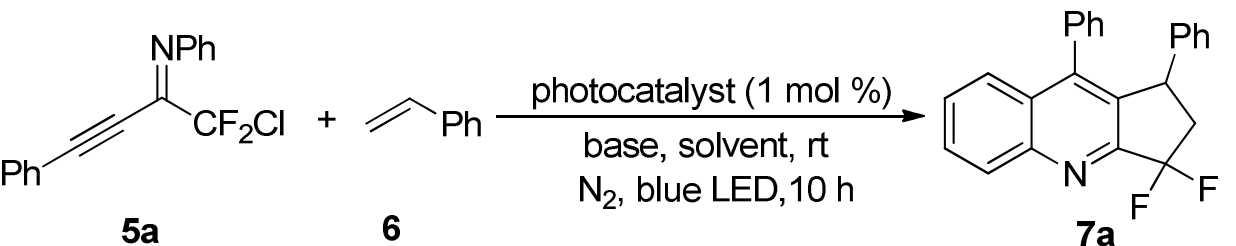

$5 a$

6

$7 a$

\begin{tabular}{llllc}
\hline entry & catalyst & base (equiv) & solvent & yield(\%) ${ }^{b}$ \\
\hline 1 & $f a c-\mathrm{Ir}(\mathrm{ppy})_{3}$ & $\mathrm{Na}_{2} \mathrm{HPO}_{4}(1.2)$ & $\mathrm{MeCN}$ & 16 \\
2 & $\mathrm{Ru}(\mathrm{bpy})_{3} \mathrm{Cl}_{2}$ & $\mathrm{Et}_{3} \mathrm{~N}(1.2)$ & $\mathrm{MeCN}$ & 68 \\
3 & $\mathrm{Ru}(\mathrm{bpy})_{3} \mathrm{Cl}_{2}$ & $n-\mathrm{Bu} \mathrm{N}_{3} \mathrm{~N}(1.2)$ & $\mathrm{MeCN}$ & 81 \\
4 & $\mathrm{Ru}(\mathrm{bpy})_{3} \mathrm{Cl}_{2}$ & $n-\mathrm{Bu}_{3} \mathrm{~N}(0.2) / \mathrm{K}_{2} \mathrm{CO}_{3}(1.2)$ & $\mathrm{MeCN}$ & 93 \\
5 & $\mathrm{Ru}(\mathrm{bpy})_{3} \mathrm{Cl}_{2}$ & $n-\mathrm{Bu}_{3} \mathrm{~N}(0.2) / \mathrm{K}_{2} \mathrm{CO}_{3}(1.2)$ & $\mathrm{DMF}$ & 84 \\
6 & $\mathrm{Ru}(\mathrm{bpy})_{3} \mathrm{Cl}_{2}$ & $n-\mathrm{Bu}_{3} \mathrm{~N}(0.2) / \mathrm{K}_{2} \mathrm{CO}_{3}(1.2)$ & $\mathrm{DMSO}$ & 79 \\
7 & $\mathrm{Ru}(\mathrm{bpy})_{3} \mathrm{Cl}_{2}$ & $n-\mathrm{Bu}_{3} \mathrm{~N}(0.2) / \mathrm{K}_{2} \mathrm{CO}_{3}(1.2)$ & toluene & 31 \\
8 & $\mathrm{Ru}(\mathrm{bpy})_{3} \mathrm{Cl}_{2}$ & $\mathrm{~K}_{2} \mathrm{CO}_{3}(1.2)$ & $\mathrm{MeCN}$ & 0 \\
9 & $n o$ catalyst & $n-\mathrm{Bu}_{3} \mathrm{~N}(0.2) / \mathrm{K}_{2} \mathrm{CO}_{3}(1.2)$ & $\mathrm{MeCN}$ & 0 \\
$10^{c}$ & $\mathrm{Ru}(\mathrm{bpy})_{3} \mathrm{Cl}_{2}$ & $n-\mathrm{Bu}_{3} \mathrm{~N}(0.2) / \mathrm{K}_{2} \mathrm{CO}_{3}(1.2)$ & $\mathrm{MeCN}$ & 0 \\
\hline
\end{tabular}

${ }^{a}$ The reaction was carried out by using $5 \mathbf{5}(0.2 \mathrm{mmol}), 6(1.2$ equiv), $1 \mathrm{~mol} \%$ of photocatalyst, and base in $1 \mathrm{~mL}$ of solvent at room temperature with the irradiation of $5 \mathrm{~W}$ blue LED for $10 \mathrm{~h} .{ }^{b}$ Yields were determined by ${ }^{1} \mathrm{H}$ NMR spectroscopy using mesitylene as the internal standard. ${ }^{c}$ The reaction was conducted in the dark.

We started our investigation with the reaction between 5a and styrene 6 (Table S1). Initially, $f a c-\operatorname{Ir}(\mathrm{ppy})_{3}$ was employed as the photocatalyst in the absence of an amine, which is the typical conditions for an oxidative quenching cycle. Only $16 \%$ yield of the desired product $7 \mathbf{a}$ was observed and $75 \%$ yield of $5 \mathbf{a}$ remained unreacted (Table $\mathrm{S} 1$, entry 1). In contrast, by utilizing the reductive quenching cycle of $\mathrm{Ru}(\mathrm{bpy})_{3} \mathrm{Cl}_{2}$ with $\mathrm{Et}_{3} \mathrm{~N}$ (1.2 equiv) as the electron donor and base, the cyclopenta-fused quinoline 7a was obtained in the yield of $68 \%$ (Table S1, entry 2). Further improvement was achieved upon changing the base from $\mathrm{Et}_{3} \mathrm{~N}$ to $n-\mathrm{Bu}_{3} \mathrm{~N}$ (Table $\mathrm{S} 1$, entry 3), which might be attributed to the stronger electron-donating ability of $n-\mathrm{Bu}_{3} \mathrm{~N}\left(E_{\text {red }}=0.78 \mathrm{~V}\right.$ 
vs. SCE $)^{[2]}$ compared with $\mathrm{Et}_{3} \mathrm{~N}\left(E_{\text {red }}=0.96 \mathrm{~V}\right.$ vs. SCE) ${ }^{[3]}$ Pleasingly, 7a was obtained in $93 \%$ yield when the mixture of $\mathrm{K}_{2} \mathrm{CO}_{3}$ (1.2 equiv) and $n$ - $\mathrm{Bu}_{3} \mathrm{~N}$ ( 0.2 equiv) was used (Table S1, entry 4). The effect of solvent for the reaction was also evaluated, and the results indicated the reaction proceeded more efficiently in polar solvents such as MeCN, DMF, and DMSO, whereas less polar solvent was found to be unfavorable (Table S1, entries 5-7). Control experiments confirmed that $n-\mathrm{Bu}_{3} \mathrm{~N}$, photocatalyst, and the continuous irradiation were all necessary for this transformation (Table S1, entries 8-10).

\section{Representative experimental procedure for the synthesis of gem-difluorinated fused} quinoline 7a: To a $10 \mathrm{~mL}$ test tube equipped with a magnetic stir bar was charged with chlorodifluoroalkynyl imine 5a $(0.2 \mathrm{mmol}, 58 \mathrm{mg})$, styrene $6(0.24 \mathrm{mmol}, 25$ $\mathrm{mg}), \mathrm{K}_{2} \mathrm{CO}_{3}(0.24 \mathrm{mmol}, 34 \mathrm{mg}), n-\mathrm{Bu}_{3} \mathrm{~N}$ (0.04 mmol, $\left.7.4 \mathrm{mg}\right), \mathrm{Ru}(\mathrm{bpy}){ }_{3} \mathrm{Cl}_{2}(1 \mathrm{~mol} \%$, $1.4 \mathrm{mg}$ ) and $1 \mathrm{~mL}$ of $\mathrm{MeCN}$. The solution was stirred at room temperature with the irradiation of a $5 \mathrm{~W}$ blue LED under argon for $10 \mathrm{~h}$. Upon completion of the reaction, the reaction mixture was filtrated through a short path of silica gel, eluted with ethyl acetate. After evaporation of the solvent, the crude product was purified by column chromatography on silica gel with hexane/ethyl acetate (60/1) as the eluant, giving the pure product $7 \mathbf{a}$ as a white solid (65 $\mathrm{mg}, 91 \%$ yield).

\section{Compound characterization data:}

\section{3,3-Difluoro-1,9-diphenyl-2,3-dihydro-1H-cyclopenta[b]quinoline (7a):}

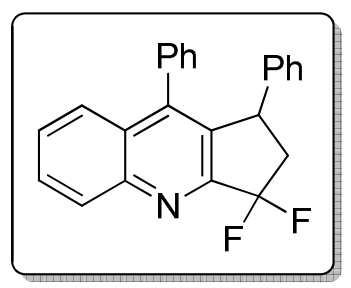

White solid (65 mg, 91\% yield); ${ }^{1} \mathrm{H}$ NMR (400 MHz, CDCl3) $\delta 8.36(\mathrm{~d}, J=8.5 \mathrm{~Hz}, 1 \mathrm{H}), 7.75(\mathrm{ddd}, J=8.4,6.7,1.4 \mathrm{~Hz}, 1 \mathrm{H})$, $7.57(\mathrm{dd}, J=8.5,0.9 \mathrm{~Hz}, 1 \mathrm{H}), 7.50-7.40(\mathrm{~m}, 2 \mathrm{H}), 7.30$ (tt, $J$ $=7.5,1.2 \mathrm{~Hz}, 1 \mathrm{H}), 7.27-7.23(\mathrm{~m}, 1 \mathrm{H}), 7.08-6.97(\mathrm{~m}, 4 \mathrm{H})$, $6.70(\mathrm{dt}, J=3.8,2.3 \mathrm{~Hz}, 2 \mathrm{H}), 6.52(\mathrm{~d}, J=7.7 \mathrm{~Hz}, 1 \mathrm{H}), 4.55(\mathrm{dt}, J=9.1,4.5 \mathrm{~Hz}, 1 \mathrm{H})$, $3.29-3.14(\mathrm{~m}, 1 \mathrm{H}), 2.80-2.66(\mathrm{~m}, 1 \mathrm{H}) ;{ }^{13} \mathrm{C} \mathrm{NMR}\left(101 \mathrm{MHz}, \mathrm{CDCl}_{3}\right) \delta 155.16(\mathrm{t}, J$ $=24.6 \mathrm{~Hz}), 149.16,147.44,143.12(\mathrm{~d}, J=1.8 \mathrm{~Hz}), 134.99(\mathrm{t}, J=4.1 \mathrm{~Hz}), 134.62$, 
$130.30,129.90,129.26,128.98,128.43,128.42,128.27,128.06,127.97,127.70$, 127.66, 126.46, 126.13 (t, $J=246.7 \mathrm{~Hz}), 125.98,44.16(\mathrm{t}, J=23.2 \mathrm{~Hz}), 43.14$ (t, $J=$ $3.2 \mathrm{~Hz}) ;{ }^{19} \mathrm{~F}$ NMR $\left(377 \mathrm{MHz}, \mathrm{CDCl}_{3}\right) \delta-89.11(\mathrm{~d}, J=259.7 \mathrm{~Hz}, 1 \mathrm{~F}),-93.31(\mathrm{~d}, J=$ $259.6 \mathrm{~Hz}, 1 \mathrm{~F})$; EI-MS (m/z, relative intensity): 357 (M+1, 86), 281 (26), 280 (100), 260 (33), 240(14); HRMS (EI) calcd. for $\mathrm{C}_{24} \mathrm{H}_{17} \mathrm{~N}_{1} \mathrm{~F}_{2}[\mathrm{M}]^{+}$357.1324, found: 357.1326.

\section{3,3-Difluoro-1-phenyl-9-(p-tolyl)-2,3-dihydro-1H-cyclopenta[b]quinoline (7b):}

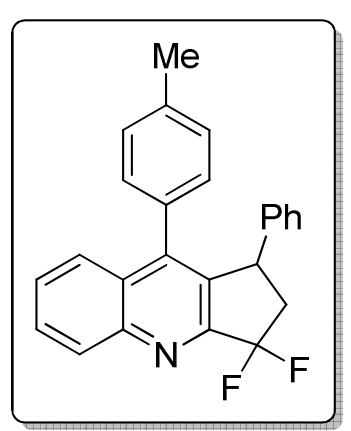

White solid (63 mg, 85\% yield); ${ }^{1} \mathrm{H}$ NMR (400 $\left.\mathrm{MHz}, \mathrm{CDCl}_{3}\right) \delta$ $8.34(\mathrm{~d}, J=8.5 \mathrm{~Hz}, 1 \mathrm{H}), 7.75(\mathrm{ddd}, J=8.4,6.8,1.4 \mathrm{~Hz}, 1 \mathrm{H})$, $7.65-7.56(\mathrm{~m}, 1 \mathrm{H}), 7.52-7.41(\mathrm{~m}, 1 \mathrm{H}), 7.23(\mathrm{~d}, J=7.6 \mathrm{~Hz}$, 1H), $7.14(\mathrm{dd}, J=7.7,1.7 \mathrm{~Hz}, 1 \mathrm{H}), 7.08-6.97$ (m, 3H), 6.86 (d, $J=7.7 \mathrm{~Hz}, 1 \mathrm{H}), 6.72(\mathrm{dd}, J=7.8,1.5 \mathrm{~Hz}, 2 \mathrm{H}), 6.41$ (dd, $J$ $=7.8,1.7 \mathrm{~Hz}, 1 \mathrm{H}), 4.54(\mathrm{dt}, J=9.0,4.4 \mathrm{~Hz}, 1 \mathrm{H}), 3.21(\mathrm{dddd}, J$ $=17.2,15.3,12.7,9.2 \mathrm{~Hz}, 1 \mathrm{H}), 2.73$ (dddd, $J=16.3,15.2,10.6,4.4 \mathrm{~Hz}, 1 \mathrm{H}), 2.36$ $(\mathrm{s}, 3 \mathrm{H}) ;{ }^{13} \mathrm{C} \mathrm{NMR}\left(101 \mathrm{MHz}, \mathrm{CDCl}_{3}\right) \delta 155.36(\mathrm{t}, J=24.4 \mathrm{~Hz}), 149.35,147.59$, 143.43, 137.95, 135.12 (t, $J=4.1 \mathrm{~Hz}), 131.77,130.50,129.85,129.29,129.24$, $129.10,128.74,128.52,128.29,127.81,127.63,126.50,126.16,126.12$ (t, $J=247.7$ $\mathrm{Hz}), 44.29$ (t, $J=23.2 \mathrm{~Hz}), 43.24(\mathrm{t}, J=3.2 \mathrm{~Hz}), 21.38 ;{ }^{19} \mathrm{~F} \mathrm{NMR}\left(377 \mathrm{MHz}, \mathrm{CDCl}_{3}\right)$ $\delta-89.09(\mathrm{~d}, J=259.4 \mathrm{~Hz}, 1 \mathrm{~F}),-93.59(\mathrm{~d}, J=259.4 \mathrm{~Hz}, 1 \mathrm{~F})$; EI-MS (m/z, relative intensity): $371\left(\mathrm{M}^{+}, 33\right), 356$ (6), 281 (24), 207 (100), 191 (13); HRMS (EI) calcd. for $\mathrm{C}_{25} \mathrm{H}_{19} \mathrm{~N}_{1} \mathrm{~F}_{2}[\mathrm{M}]^{+}$371.1480, found: 371.1481 .

\section{3,3-Difluoro-9-(4-methoxyphenyl)-1-phenyl-2,3-dihydro-1 $H$-cyclopenta[b]quinoli} ne (7c):

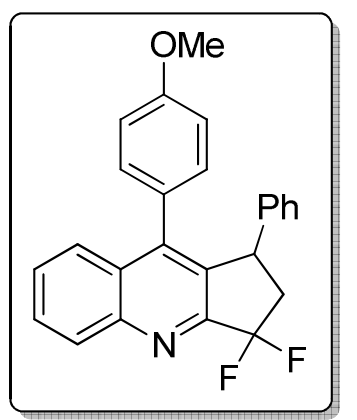

White solid (61 mg, 79\% yield); ${ }^{1} \mathrm{H} \mathrm{NMR}\left(400 \mathrm{MHz}, \mathrm{CDCl}_{3}\right) \delta$ $8.23(\mathrm{~d}, J=9.3 \mathrm{~Hz}, 1 \mathrm{H}), 7.47-7.38(\mathrm{~m}, 2 \mathrm{H}), 7.32-7.22(\mathrm{~m}$, 2H), $7.07-6.97(\mathrm{~m}, 4 \mathrm{H}), 6.77(\mathrm{~d}, J=2.8 \mathrm{~Hz}, 1 \mathrm{H}), 6.68(\mathrm{dd}, J$ $=7.8,1.5 \mathrm{~Hz}, 2 \mathrm{H}), 6.52(\mathrm{~d}, J=7.7 \mathrm{~Hz}, 1 \mathrm{H}), 4.50(\mathrm{dt}, J=9.1$, $4.5 \mathrm{~Hz}, 1 \mathrm{H}), 3.67(\mathrm{~s}, 3 \mathrm{H}), 3.28-3.11(\mathrm{~m}, 1 \mathrm{H}), 2.78-2.63(\mathrm{~m}$, 
$1 \mathrm{H}) ;{ }^{13} \mathrm{C} \mathrm{NMR}\left(101 \mathrm{MHz}, \mathrm{CDCl}_{3}\right) \delta 159.57,155.42(\mathrm{t}, J=24.4 \mathrm{~Hz}), 149.50,147.30$, 143.50, $135.28(\mathrm{t}, J=3.7 \mathrm{~Hz}), 130.73,130.55,129.92,129.83,129.43,128.37$, 127.79, 127.64, 127.02, 126.58, 126.12, 126.10 (t, $J=247.2 \mathrm{~Hz}), 114.08,113.62$, $55.47,44.34(\mathrm{t}, J=23.2 \mathrm{~Hz}), 43.32 ;{ }^{19} \mathrm{~F}$ NMR $\left(377 \mathrm{MHz}, \mathrm{CDCl}_{3}\right) \delta-88.49(\mathrm{~d}, J=$ $258.0 \mathrm{~Hz}, 1 \mathrm{~F}),-92.84(\mathrm{~d}, J=258.0 \mathrm{~Hz}, 1 \mathrm{~F})$; EI-MS (m/z, relative intensity): $387\left(\mathrm{M}^{+}\right.$, 23), 341 (6), 281 (28), 207 (100), 133 (12); HRMS (EI) calcd. for $\mathrm{C}_{25} \mathrm{H}_{19} \mathrm{O}_{1} \mathrm{~N}_{1} \mathrm{~F}_{2}[\mathrm{M}]^{+}$ 387.1429, found: 387.1427 .

\section{3,3-Difluoro-9-(4-fluorophenyl)-1-phenyl-2,3-dihydro-1 $H$-cyclopenta[b]quinoline} (7d):

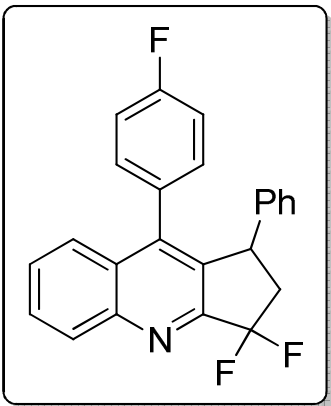

White solid (64 mg, 85\% yield); ${ }^{1} \mathrm{H}$ NMR (400 MHz, $\left.\mathrm{CDCl}_{3}\right) \delta$ $8.35(\mathrm{~d}, J=8.5 \mathrm{~Hz}, 1 \mathrm{H}), 7.77(\mathrm{ddd}, J=8.4,6.3,1.9 \mathrm{~Hz}, 1 \mathrm{H})$, $7.57-7.44(\mathrm{~m}, 2 \mathrm{H}), 7.23(\mathrm{ddd}, J=7.8,5.3,2.2 \mathrm{~Hz}, 1 \mathrm{H}), 7.13$ $(\mathrm{td}, J=8.5,2.7 \mathrm{~Hz}, 1 \mathrm{H}), 7.09-7.00(\mathrm{~m}, 3 \mathrm{H}), 6.77-6.67(\mathrm{~m}$, $3 \mathrm{H}), 6.51-6.43(\mathrm{~m}, 1 \mathrm{H}), 4.52(\mathrm{dt}, J=9.1,4.6 \mathrm{~Hz}, 1 \mathrm{H}), 3.23$ (dddd, $J=17.2,15.3,11.3,9.2 \mathrm{~Hz}, 1 \mathrm{H}), 2.73$ (tdd, $J=16.6$,

12.0, 4.8 Hz, 1H); ${ }^{13} \mathrm{C}$ NMR (101 MHz, $\left.\mathrm{CDCl}_{3}\right) \delta 162.58(\mathrm{~d}, J=248.0 \mathrm{~Hz}), 155.42(\mathrm{t}$, $J=24.6 \mathrm{~Hz}), 149.39,146.32,143.18(\mathrm{~d}, J=1.5 \mathrm{~Hz}), 135.32(\mathrm{t}, J=4.1 \mathrm{~Hz}), 131.25$ $(\mathrm{d}, J=8.4 \mathrm{~Hz}), 130.70(\mathrm{~d}, J=3.7 \mathrm{~Hz}), 130.65,130.32(\mathrm{~d}, J=8.2 \mathrm{~Hz}), 130.04,129.11$, 128.50, 127.94, 127.78, 126.76, 125.94 (dd, $J=248.2,246.3 \mathrm{~Hz}), 125.76,115.60$ (d, $J=21.7 \mathrm{~Hz}), 115.22(\mathrm{~d}, J=21.6 \mathrm{~Hz}), 44.32(\mathrm{t}, J=23.3 \mathrm{~Hz}), 43.30(\mathrm{t}, J=3.2 \mathrm{~Hz})$; ${ }^{19} \mathrm{~F}$ NMR $\left(377 \mathrm{MHz}, \mathrm{CDCl}_{3}\right) \delta-89.93(\mathrm{~d}, J=259.8 \mathrm{~Hz}, 1 \mathrm{~F}),-93.28(\mathrm{~d}, J=259.8 \mathrm{~Hz}$, 1F), -113.46 (s, 1F); EI-MS (m/z, relative intensity): $375\left(\mathrm{M}^{+}, 18\right), 355$ (8), 281 (29), 207 (100), 191 (14); HRMS (EI) calcd. for $\mathrm{C}_{24} \mathrm{H}_{16} \mathrm{~N}_{1} \mathrm{~F}_{3}[\mathrm{M}]^{+}$375.1229, found: 375.1227.

\section{9-(4-Chlorophenyl)-3,3-difluoro-1-phenyl-2,3-dihydro-1H-cyclopenta[b]quinoline} (7e):

White solid (68 mg, 87\% yield); ${ }^{1} \mathrm{H}$ NMR (400 MHz, $\left.\mathrm{CDCl}_{3}\right) \delta 8.35(\mathrm{~d}, J=8.5 \mathrm{~Hz}$, 1H), 7.77 (ddd, $J=8.4,5.8,2.4 \mathrm{~Hz}, 1 \mathrm{H}), 7.53-7.48(\mathrm{~m}, 2 \mathrm{H}), 7.41(\mathrm{dd}, J=8.2,2.2$ 


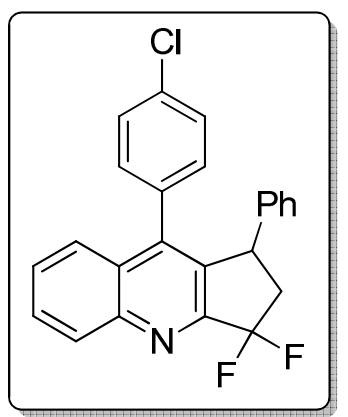

$\mathrm{Hz}, 1 \mathrm{H}), 7.19(\mathrm{dd}, J=8.2,2.1 \mathrm{~Hz}, 1 \mathrm{H}), 7.09-6.99(\mathrm{~m}, 4 \mathrm{H})$, $6.72(\mathrm{dd}, J=7.8,1.6 \mathrm{~Hz}, 2 \mathrm{H}), 6.43(\mathrm{dd}, J=8.2,2.1 \mathrm{~Hz}, 1 \mathrm{H})$, 4.51 (dt, $J=9.1,4.6 \mathrm{~Hz}, 1 \mathrm{H}), 3.23$ (dddd, $J=17.2,15.3,11.3$, $9.2 \mathrm{~Hz}, 1 \mathrm{H}), 2.87-2.60(\mathrm{~m}, 1 \mathrm{H}) ;{ }^{13} \mathrm{C} \mathrm{NMR}\left(101 \mathrm{MHz}, \mathrm{CDCl}_{3}\right)$ $\delta 155.41(\mathrm{t}, J=24.6 \mathrm{~Hz}), 149.35,146.02,143.08,135.18$ (t, $J$ $=4.2 \mathrm{~Hz}), 134.33,133.22,130.73,130.66,130.07,129.97$, $128.84,128.75,128.51,128.32,128.00,127.81,126.79$, 125.87 (dd, J =248.6, $246.4 \mathrm{~Hz}), 125.69,44.29(\mathrm{t}, J=23.3 \mathrm{~Hz}), 43.26(\mathrm{t}, J=3.2 \mathrm{~Hz}) ;{ }^{19} \mathrm{~F}$ NMR $(377 \mathrm{MHz}$, $\left.\mathrm{CDCl}_{3}\right) \delta-90.00(\mathrm{~d}, J=259.9 \mathrm{~Hz}, 1 \mathrm{~F}),-93.31(\mathrm{~d}, J=259.7 \mathrm{~Hz}, 1 \mathrm{~F}) ;$ EI-MS (m/z, relative intensity): $393\left({ }^{37} \mathrm{Cl}, \mathrm{M}^{+}, 55\right), 391\left({ }^{35} \mathrm{Cl}, \mathrm{M}^{+}, 17\right), 356(37), 280$ (45), $207(100)$, 167 (24); HRMS (EI) calcd. for $\mathrm{C}_{24} \mathrm{H}_{16} \mathrm{~N}_{1} \mathrm{Cl}_{1} \mathrm{~F}_{2}[\mathrm{M}]^{+}$391.0934, found: 391.0932.

\section{9-(4-Bromophenyl)-3,3-difluoro-1-phenyl-2,3-dihydro-1 $H$-cyclopenta[b]quinoline} (7f):

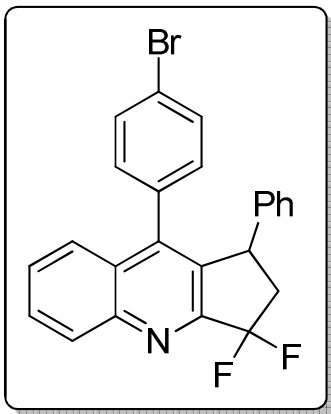

White solid (74 mg, 85\% yield); ${ }^{1} \mathrm{H}$ NMR (400 $\left.\mathrm{MHz}, \mathrm{CDCl}_{3}\right) \delta$ $8.35(\mathrm{~d}, J=8.5 \mathrm{~Hz}, 1 \mathrm{H}), 7.81-7.73(\mathrm{~m}, 1 \mathrm{H}), 7.57(\mathrm{dd}, J=8.1$, $2.0 \mathrm{~Hz}, 1 \mathrm{H}), 7.53-7.45(\mathrm{~m}, 2 \mathrm{H}), 7.19-7.10(\mathrm{~m}, 2 \mathrm{H}), 7.10-$ $7.01(\mathrm{~m}, 3 \mathrm{H}), 6.73(\mathrm{dt}, J=3.6,2.2 \mathrm{~Hz}, 2 \mathrm{H}), 6.36(\mathrm{dd}, J=8.2$, $2.1 \mathrm{~Hz}, 1 \mathrm{H}), 4.50(\mathrm{dt}, J=9.1,4.6 \mathrm{~Hz}, 1 \mathrm{H}), 3.23$ (dddd, $J=$ $17.1,15.3,11.2,9.2 \mathrm{~Hz}, 1 \mathrm{H}), 2.82-2.66(\mathrm{~m}, 1 \mathrm{H}) ;{ }^{13} \mathrm{C} \mathrm{NMR}$ $\left(101 \mathrm{MHz}, \mathrm{CDCl}_{3}\right) \delta 155.42(\mathrm{t}, J=24.6 \mathrm{~Hz}), 149.35,145.99,143.11,135.12(\mathrm{t}, J=$ $4.2 \mathrm{~Hz}), 133.72$, 131.70, 131.28, 131.00, 130.67, 130.26, 130.08, 128.76, 128.52, $128.02,127.83,126.80,125.87(\mathrm{dd}, J=248.2,246.4 \mathrm{~Hz}), 125.68,122.50,44.29(\mathrm{t}, J$ $=23.3 \mathrm{~Hz}), 43.27(\mathrm{t}, J=3.2 \mathrm{~Hz}) ;{ }^{19} \mathrm{~F} \mathrm{NMR}\left(377 \mathrm{MHz}, \mathrm{CDCl}_{3}\right) \delta-90.05(\mathrm{~d}, J=259.8$ $\mathrm{Hz}, 1 \mathrm{~F}),-93.32$ (d, $J=259.9 \mathrm{~Hz}, 1 \mathrm{~F})$; EI-MS (m/z, relative intensity): $437\left({ }^{81} \mathrm{Br} \mathrm{M}^{+}\right.$, 40), 435 ( $\left.{ }^{79} \mathrm{Br}, \mathrm{M}^{+}, 40\right), 356$ (46), 280 (47), 207 (100), 168 (3); HRMS (EI) calcd. for $\mathrm{C}_{24} \mathrm{H}_{16} \mathrm{~N}_{1} \mathrm{Br}_{1} \mathrm{~F}_{2}[\mathrm{M}]^{+} 435.0429$, found: 435.0426 .

\section{1-(4-(3,3-Difluoro-1-phenyl-2,3-dihydro-1H-cyclopenta[b]quinolin-9-yl)phenyl)et} hanone (7g): 


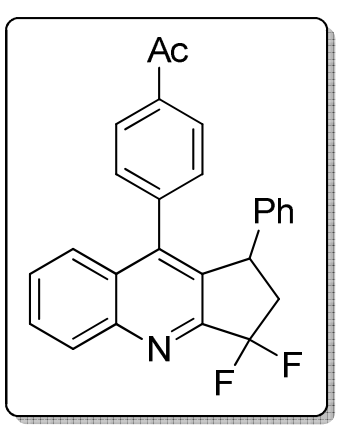

White solid (70 mg, 87\% yield); ${ }^{1} \mathrm{H}$ NMR (400 MHz, $\left.\mathrm{CDCl}_{3}\right) \delta$ $8.36(\mathrm{~d}, J=8.6 \mathrm{~Hz}, 1 \mathrm{H}), 8.02(\mathrm{dd}, J=7.9,1.6 \mathrm{~Hz}, 1 \mathrm{H}), 7.77$ (ddd, $J=8.4,6.2,2.0 \mathrm{~Hz}, 1 \mathrm{H}), 7.61(\mathrm{dd}, J=8.0,1.6 \mathrm{~Hz}, 1 \mathrm{H})$, $7.52-7.44(\mathrm{~m}, 2 \mathrm{H}), 7.37(\mathrm{dd}, J=7.9,1.5 \mathrm{~Hz}, 1 \mathrm{H}), 7.07-6.96$ (m, 3H), $6.69(\mathrm{dd}, J=5.1,3.3 \mathrm{~Hz}, 2 \mathrm{H}), 6.62(\mathrm{dd}, J=8.0,1.5$ $\mathrm{Hz}, 1 \mathrm{H}), 4.51$ (dt, $J=9.1,4.6 \mathrm{~Hz}, 1 \mathrm{H}), 3.23$ (dddd, $J=17.1$, 15.3, 11.1, 9.2 Hz, 1H), $2.81-2.65(\mathrm{~m}, 1 \mathrm{H}), 2.62$ (s, 3H); ${ }^{13} \mathrm{C}$ NMR (101 MHz, $\left.\mathrm{CDCl}_{3}\right) \delta 197.69,155.38(\mathrm{t}, J=24.6 \mathrm{~Hz}), 149.23,146.11,142.95,139.76,136.62$, $134.91(\mathrm{t}, J=4.2 \mathrm{~Hz}), 130.64,130.19,129.76,128.98,128.53,128.47,128.42$, $128.13,127.96,127.83,126.80,125.79$ (dd, $J=248.2,246.4 \mathrm{~Hz}), 125.62,44.33$ (t, $J$ $=23.3 \mathrm{~Hz}), 43.25(\mathrm{t}, J=3.1 \mathrm{~Hz}), 26.82 ;{ }^{19} \mathrm{~F}$ NMR $\left(377 \mathrm{MHz}, \mathrm{CDCl}_{3}\right) \delta-90.12(\mathrm{~d}, J=$ $260.1 \mathrm{~Hz}, 1 \mathrm{~F}),-93.27$ (d, $J=260.1 \mathrm{~Hz}, 1 \mathrm{~F})$; EI-MS (m/z, relative intensity): $399\left(\mathrm{M}^{+}\right.$, 8), 341 (6), 281 (27), 207 (100), 191 (12), 147(10); HRMS (EI) calcd. for $\mathrm{C}_{26} \mathrm{H}_{19} \mathrm{O}_{1} \mathrm{~N}_{1} \mathrm{~F}_{2}[\mathrm{M}]^{+}$399.1429, found: 399.1426.

\section{3,3-Difluoro-9-(4-nitrophenyl)-1-phenyl-2,3-dihydro-1 $H$-cyclopenta[b]quinoline}

(7h):

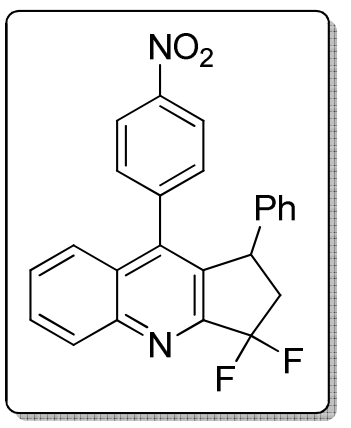

Yellow solid (60 mg, 75\% yield); ${ }^{1} \mathrm{H} \mathrm{NMR}\left(400 \mathrm{MHz}, \mathrm{CDCl}_{3}\right)$ $\delta 8.38(\mathrm{~d}, J=8.5 \mathrm{~Hz}, 1 \mathrm{H}), 8.29(\mathrm{dd}, J=8.4,2.3 \mathrm{~Hz}, 1 \mathrm{H}), 7.87$ (dd, $J=8.4,2.3 \mathrm{~Hz}, 1 \mathrm{H}), 7.80(\mathrm{ddd}, J=8.4,6.8,1.3 \mathrm{~Hz}, 1 \mathrm{H})$, $7.52(\mathrm{ddd}, J=8.1,6.9,1.1 \mathrm{~Hz}, 1 \mathrm{H}), 7.45(\mathrm{dd}, J=8.4,1.8 \mathrm{~Hz}$, 1H), 7.39 (dd, $J=8.5,0.7 \mathrm{~Hz}, 1 \mathrm{H}), 7.08$ (ddd, $J=6.2,3.6,1.3$ $\mathrm{Hz}, 1 \mathrm{H}), 7.04-6.98(\mathrm{~m}, 2 \mathrm{H}), 6.73-6.65(\mathrm{~m}, 3 \mathrm{H}), 4.55-4.45$

(m, 1H), $3.34-3.17(\mathrm{~m}, 1 \mathrm{H}), 2.75(\mathrm{tdd}, J=15.5,13.9,5.5 \mathrm{~Hz}, 1 \mathrm{H}) ;{ }^{13} \mathrm{C}$ NMR $(101$ $\left.\mathrm{MHz}, \mathrm{CDCl}_{3}\right) \delta 155.53(\mathrm{t}, J=24.7 \mathrm{~Hz}), 149.29,147.60,144.62,142.70,141.77$, $134.99(\mathrm{t}, J=4.2 \mathrm{~Hz}), 130.90,130.59,130.39,129.74,128.68,128.49,128.17$, $127.87,127.13,125.59$ (dd, $J=248.7,246.1 \mathrm{~Hz}), 125.20,123.73,123.09,44.40$ (t, $J$ $=23.4 \mathrm{~Hz}), 43.30(\mathrm{t}, J=3.1 \mathrm{~Hz}) ;{ }^{19} \mathrm{~F} \mathrm{NMR}\left(377 \mathrm{MHz}, \mathrm{CDCl}_{3}\right) \delta-91.26(\mathrm{~d}, J=260.2$ $\mathrm{Hz}, 1 \mathrm{~F}),-93.10$ (d, $J=260.3 \mathrm{~Hz}, 1 \mathrm{~F})$; EI-MS (m/z, relative intensity): $402\left(\mathrm{M}^{+}, 4\right)$, 
341 (8), 281 (28), 267 (5), 207 (100), 191(14); HRMS (EI) calcd. for $\mathrm{C}_{24} \mathrm{H}_{16} \mathrm{O}_{2} \mathrm{~N}_{2} \mathrm{~F}_{2}$ $[\mathrm{M}]^{+}$402.1174, found: 402.1172 .

3,3-Difluoro-1-phenyl-9-(m-tolyl)-2,3-dihydro-1 $H$-cyclopenta[b]quinolone

(7i),

\section{4:1 mixture of two diastereomers:}

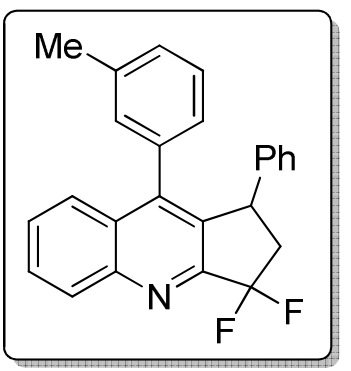

White solid (61 mg, 83\% yield); ${ }^{1} \mathrm{H}$ NMR (400 MHz, $\left.\mathrm{CDCl}_{3}\right) \delta$ $8.34(\mathrm{~d}, J=8.5 \mathrm{~Hz}, 1 \mathrm{H}), 8.34\left(\mathrm{~d}, J=8.5 \mathrm{~Hz}, 1 \mathrm{H}^{\prime}\right), 7.83-7.67$ (m, 1H), $7.83-7.67\left(\mathrm{~m}, 1 \mathrm{H}^{\prime}\right), 7.58$ (ddd, $J=16.3,8.5,0.9 \mathrm{~Hz}$, $1 \mathrm{H}), 7.58\left(\mathrm{ddd}, J=16.3,8.5,0.9 \mathrm{~Hz}, 1 \mathrm{H}^{\prime}\right), 7.51-7.39(\mathrm{~m}, 1 \mathrm{H})$, $7.51-7.39\left(\mathrm{~m}, 1 \mathrm{H}^{\prime}\right), 7.31(\mathrm{t}, J=7.6 \mathrm{~Hz}, 1 \mathrm{H}), 7.12-7.08(\mathrm{~m}$, 1H), $7.12-7.08\left(\mathrm{~m}, 1 \mathrm{H}^{\prime}\right), 7.12-7.08\left(\mathrm{~m}, 1 \mathrm{H}^{\prime}\right), 7.07-7.00(\mathrm{~m}, 4 \mathrm{H}), 7.07$ - $7.00(\mathrm{~m}$, $\left.4 \mathrm{H}^{\prime}\right), 6.94\left(\mathrm{t}, J=7.6 \mathrm{~Hz}, 1 \mathrm{H}^{\prime}\right), 6.74-6.68(\mathrm{~m}, 2 \mathrm{H}), 6.74-6.68\left(\mathrm{~m}, 2 \mathrm{H}^{\prime}\right), 6.34(\mathrm{~d}, J=$ $\left.7.6 \mathrm{~Hz}, 1 \mathrm{H}^{\prime}\right), 6.19(\mathrm{~s}, 1 \mathrm{H}), 4.56\left(\mathrm{dt}, J=9.0,4.5 \mathrm{~Hz}, 1 \mathrm{H}^{\prime}\right), 4.47(\mathrm{dt}, J=9.1,4.5 \mathrm{~Hz}$, $1 \mathrm{H}), 3.31-3.11(\mathrm{~m}, 1 \mathrm{H}), 3.31-3.11\left(\mathrm{~m}, 1 \mathrm{H}^{\prime}\right), 2.82-2.64(\mathrm{~m}, 1 \mathrm{H}), 2.82-2.64(\mathrm{~m}$,

1H'), $2.40\left(\mathrm{~s}, 3 \mathrm{H}^{\prime}\right), 2.03(\mathrm{~s}, 3 \mathrm{H}) ;{ }^{13} \mathrm{C} \mathrm{NMR}\left(101 \mathrm{MHz}, \mathrm{CDCl}_{3}\right) \delta 155.43(\mathrm{t}, J=24.3$ Hz), 155.31 (t, $J=24.4 \mathrm{~Hz}), 149.42,147.70,147.55,143.59$, 143.28, 138.11, 137.78, $135.20(\mathrm{t}, J=3.7 \mathrm{~Hz}), 134.89(\mathrm{t}, J=4.0 \mathrm{~Hz}), 130.53,130.51,130.08,129.88,129.25$, $129.18,129.11,128.81,128.35,128.26,128.00,127.90,127.80,127.66,126.59$, 126.54, 126.50, 126.20, $126.16(\mathrm{t}, J=247.7 \mathrm{~Hz}), 126.07$ (t, $J=247.8 \mathrm{~Hz}), 125.50$, 44.30 (q, $J=22.7 \mathrm{~Hz}$ ), 21.50, 21.24; ${ }^{19} \mathrm{~F}$ NMR (377 MHz, $\left.\mathrm{CDCl}_{3}\right) \delta-89.09$ (d, $J=$ $259.5 \mathrm{~Hz}, 1 \mathrm{~F}),-89.59$ (d, $\left.J=259.4 \mathrm{~Hz}, 1 \mathrm{~F}^{\prime}\right),-93.39$ (d, $\left.J=259.5 \mathrm{~Hz}, 1 \mathrm{~F}\right),-93.49$ (d, $J$ $\left.=259.4 \mathrm{~Hz}, 1 \mathrm{~F}^{\prime}\right)$; EI-MS (m/z, relative intensity): $371\left(\mathrm{M}^{+}, 100\right), 351$ (17), 280 (87), 260 (26), 207 (90); HRMS (EI) calcd. for $\mathrm{C}_{25} \mathrm{H}_{19} \mathrm{~N}_{1} \mathrm{~F}_{2}[\mathrm{M}]^{+}$371.1480, found: 371.1479 .

\section{3,3-Difluoro-9-(naphthalen-1-yl)-1-phenyl-2,3-dihydro-1H-cyclopenta[b]quinolin}

\section{e (7j), 1.3:1 mixture of two diastereomers:}

White solid (68 mg, 85\% yield); ${ }^{1} \mathrm{H}$ NMR (400 MHz, $\left.\mathrm{CDCl}_{3}\right) \delta 8.40(\mathrm{t}, J=8.1 \mathrm{~Hz}$, 1H), $8.40\left(\mathrm{t}, J=8.1 \mathrm{~Hz}, 1 \mathrm{H}^{\prime}\right), 7.93(\mathrm{~d}, J=8.2 \mathrm{~Hz}, 1 \mathrm{H}), 7.84$ (d, $\left.J=8.3 \mathrm{~Hz}, 1 \mathrm{H}^{\prime}\right), 7.80$ $-7.76(\mathrm{~m}, 1 \mathrm{H}), 7.80-7.76\left(\mathrm{~m}, 1 \mathrm{H}^{\prime}\right), 7.75-7.71(\mathrm{~m}, 1 \mathrm{H}), 7.69\left(\mathrm{~d}, J=8.2 \mathrm{~Hz}, 1 \mathrm{H}^{\prime}\right)$, 


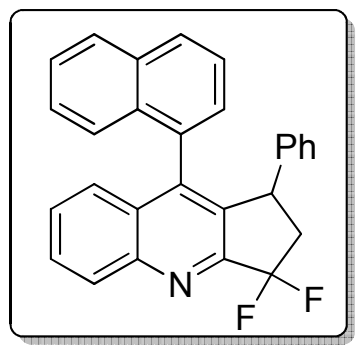

$7.57-7.50(\mathrm{~m}, 2 \mathrm{H}), 7.48(\mathrm{dd}, J=8.5,1.1 \mathrm{~Hz}, 1 \mathrm{H}), 7.44-$ $7.37\left(\mathrm{~m}, 2 \mathrm{H}^{\prime}\right), 7.37-7.31(\mathrm{~m}, 1 \mathrm{H}), 7.37-7.31\left(\mathrm{~m}, 1 \mathrm{H}^{\prime}\right), 7.29$ - $7.23(\mathrm{~m}, 1 \mathrm{H}), 7.29-7.23\left(\mathrm{~m}, 1 \mathrm{H}^{\prime}\right), 7.19\left(\mathrm{~d}, J=8.4 \mathrm{~Hz}, 1 \mathrm{H}^{\prime}\right)$, $7.08(\mathrm{dd}, J=8.2,7.1 \mathrm{~Hz}, 1 \mathrm{H}), 7.04-6.95\left(\mathrm{~m}, 1 \mathrm{H}^{\prime}\right), 6.93-$ $6.87(\mathrm{~m}, 2 \mathrm{H}), 6.93-6.87\left(\mathrm{~m}, 1 \mathrm{H}^{\prime}\right), 6.56(\mathrm{dd}, J=7.0,1.0 \mathrm{~Hz}$, $1 \mathrm{H}), 6.54-6.48(\mathrm{~m}, 1 \mathrm{H}), 6.54-6.48\left(\mathrm{~m}, 3 \mathrm{H}^{\prime}\right), 6.45(\mathrm{dt}, J=7.3,1.8 \mathrm{~Hz}, 2 \mathrm{H}), 6.39-$ $6.34\left(\mathrm{~m}, 2 \mathrm{H}^{\prime}\right), 4.59-4.45(\mathrm{~m}, 1 \mathrm{H}), 4.17\left(\mathrm{dt}, J=9.3,4.7 \mathrm{~Hz}, 1 \mathrm{H}^{\prime}\right), 3.35-3.07(\mathrm{~m}, 1 \mathrm{H})$, $3.35-3.07\left(\mathrm{~m}, 1 \mathrm{H}^{\prime}\right), 2.75(\mathrm{qdd}, J=15.4,12.8,5.2 \mathrm{~Hz}, 1 \mathrm{H}), 2.75(\mathrm{qdd}, J=15.4,12.8$, $5.2 \mathrm{~Hz}, 1 \mathrm{H}) ;{ }^{13} \mathrm{C} \mathrm{NMR}\left(101 \mathrm{MHz}, \mathrm{CDCl}_{3}\right) \delta 155.45(\mathrm{t}, J=24.4 \mathrm{~Hz}), 155.17(\mathrm{t}, J=$ $24.5 \mathrm{~Hz}), 149.56,149.02,146.16,145.69,142.86,142.84,141.13,141.12,136.50$ (t, $J$ $=4.2 \mathrm{~Hz}), 136.07(\mathrm{t}, J=4.1 \mathrm{~Hz}), 133.51,133.05,132.86,131.55,131.17,131.08$, $130.56,130.12$, 129.98, 129.73, 129.58, 129.11, 128.78, 128.47, 128.03, 127.89, $127.75,127.63,127.39,127.32,126.95,126.54,126.47,126.41,126.21,126.15$, $126.08(\mathrm{t}, J=248.1 \mathrm{~Hz}), 126.06(\mathrm{t}, \mathrm{J}=245.9 \mathrm{~Hz}), 125.93,125.89,125.85,125.80$, $125.13,124.93,124.83,44.11(\mathrm{td}, J=23.4,16.7 \mathrm{~Hz}), 43.61(\mathrm{t}, J=3.2 \mathrm{~Hz}), 43.43(\mathrm{t}, J$ $=3.2 \mathrm{~Hz}) ;{ }^{19} \mathrm{~F}$ NMR $\left(377 \mathrm{MHz}, \mathrm{CDCl}_{3}\right) \delta-89.97(\mathrm{~d}, J=259.7 \mathrm{~Hz}, 1 \mathrm{~F}),-90.07(\mathrm{~d}, J=$ $\left.259.9 \mathrm{~Hz}, 1 \mathrm{~F}^{\prime}\right),-92.60(\mathrm{~d}, J=260.0 \mathrm{~Hz}, 1 \mathrm{~F}),-93.10$ (d, $\left.J=259.7 \mathrm{~Hz}, 1 \mathrm{~F}^{\prime}\right)$; EI-MS (m/z, relative intensity): $407\left(\mathrm{M}^{+}, 17\right), 371$ (100), 356 (53), 294 (66), 279 (28), 185 (37); HRMS (EI) calcd. for $\mathrm{C}_{28} \mathrm{H}_{19} \mathrm{~N}_{1} \mathrm{~F}_{2}[\mathrm{M}]^{+} 407.1480$, found: 407.1483 .

trans-9-(2-Bromophenyl)-3,3-difluoro-1-phenyl-2,3-dihydro-1H-cyclopenta[b]qui noline (7k):

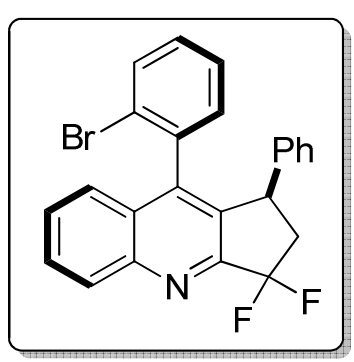

White solid (49 mg, 57\% yield); ${ }^{1} \mathrm{H} \mathrm{NMR}\left(400 \mathrm{MHz}, \mathrm{CDCl}_{3}\right)$ $\delta 8.37(\mathrm{~d}, J=8.5 \mathrm{~Hz}, 1 \mathrm{H}), 7.77(\mathrm{ddd}, J=8.4,6.8,1.4 \mathrm{~Hz}, 1 \mathrm{H})$, $7.64(\mathrm{dd}, J=8.1,1.0 \mathrm{~Hz}, 1 \mathrm{H}), 7.48(\mathrm{ddd}, J=8.1,6.8,1.1 \mathrm{~Hz}$, 1H), $7.36(\mathrm{dd}, J=8.5,0.8 \mathrm{~Hz}, 1 \mathrm{H}), 7.20-\mathrm{S} 7.10(\mathrm{~m}, 1 \mathrm{H}), 7.07$ $-6.95(\mathrm{~m}, 3 \mathrm{H}), 6.90(\mathrm{td}, J=7.5,1.1 \mathrm{~Hz}, 1 \mathrm{H}), 6.77(\mathrm{t}, J=1.9$

$\mathrm{Hz}, 1 \mathrm{H}), 6.75(\mathrm{t}, J=1.8 \mathrm{~Hz}, 1 \mathrm{H}), 6.38(\mathrm{dd}, J=7.6,1.6 \mathrm{~Hz}, 1 \mathrm{H}), 4.58-4.50(\mathrm{~m}, 1 \mathrm{H})$, $3.40-3.18(\mathrm{~m}, 1 \mathrm{H}), 2.77(\mathrm{dddd}, J=16.5,15.3,13.0,5.4 \mathrm{~Hz}, 1 \mathrm{H}) ;{ }^{13} \mathrm{C}$ NMR $(101$ $\left.\mathrm{MHz}, \mathrm{CDCl}_{3}\right) \delta 155.39(\mathrm{t}, J=24.6 \mathrm{~Hz}), 149.46,145.64,143.00,135.64(\mathrm{t}, J=3.9$ 
Hz), 135.46, 132.61, 131.33, 130.70, 130.08, 129.78, 128.61, 128.44, 128.07, 127.71, $126.85,126.77,125.94(\mathrm{t}, J=247.1 \mathrm{~Hz}), 125.44,122.53,44.21(\mathrm{t}, J=23.4 \mathrm{~Hz})$, 43.66; ${ }^{19} \mathrm{~F}$ NMR $\left(377 \mathrm{MHz}, \mathrm{CDCl}_{3}\right) \delta-90.14(\mathrm{~d}, J=259.7 \mathrm{~Hz}, 1 \mathrm{~F}),-93.59$ (d, $J=$ $259.7 \mathrm{~Hz}, 1 \mathrm{~F})$; EI-MS (m/z, relative intensity): $437\left({ }^{81} \mathrm{Br}, \mathrm{M}^{+}, 35\right), 435\left({ }^{79} \mathrm{Br}, \mathrm{M}^{+}, 35\right)$, 356 (54), 336 (23), 280 (40), 207 (100); HRMS (EI) calcd. for $\mathrm{C}_{24} \mathrm{H}_{16} \mathrm{~N}_{1} \mathrm{Br}_{1} \mathrm{~F}_{2}[\mathrm{M}]^{+}$ 435.0429, found: 435.0427 .

cis-9-(2-Bromophenyl)-3,3-difluoro-1-phenyl-2,3-dihydro-1H-cyclopenta[b]quino line (7k'):

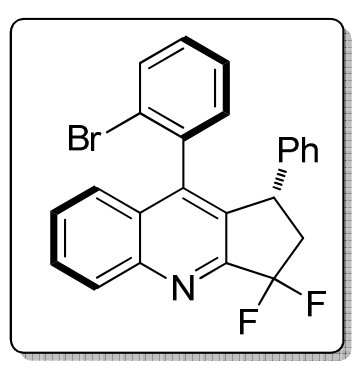

White solid (20 mg, 23\% yield); ${ }^{1} \mathrm{H}$ NMR (400 MHz, $\mathrm{CDCl}_{3}$ ) $\delta 8.35(\mathrm{~d}, J=8.5 \mathrm{~Hz}, 1 \mathrm{H}), 7.76(\mathrm{ddd}, J=8.4,6.7,1.4 \mathrm{~Hz}, 1 \mathrm{H})$, $7.56-7.47(\mathrm{~m}, 1 \mathrm{H}), 7.44-7.39(\mathrm{~m}, 2 \mathrm{H}), 7.30(\mathrm{dd}, J=8.1$, $1.0 \mathrm{~Hz}, 1 \mathrm{H}), 7.24(\mathrm{dd}, J=7.6,1.6 \mathrm{~Hz}, 1 \mathrm{H}), 7.21-7.15(\mathrm{~m}$, 1H), $7.06-6.99(\mathrm{~m}, 3 \mathrm{H}), 6.75(\mathrm{t}, J=2.1 \mathrm{~Hz}, 1 \mathrm{H}), 6.73(\mathrm{t}, J=$

$1.9 \mathrm{~Hz}, 1 \mathrm{H}), 4.63-4.32(\mathrm{~m}, 1 \mathrm{H}), 3.24$ (ddt, $J=17.2,15.4,9.3 \mathrm{~Hz}, 1 \mathrm{H}), 2.91-2.67$ $(\mathrm{m}, 1 \mathrm{H}) ;{ }^{13} \mathrm{C} \mathrm{NMR}\left(101 \mathrm{MHz}, \mathrm{CDCl}_{3}\right) \delta 155.61(\mathrm{t}, J=27.7 \mathrm{~Hz}), 149.15,145.76$, 140.97, 135.90, $134.68(\mathrm{t}, J=3.8 \mathrm{~Hz}), 132.97,130.71,130.44,130.05,128.46$, 128.08, 127.98, 127.60, 127.60, 126.74, 126.10, 125.89 (t, $J=248.4 \mathrm{~Hz}$ ), 123.87, $44.36(\mathrm{t}, J=23.4 \mathrm{~Hz}), 43.44 ;{ }^{19} \mathrm{~F} \mathrm{NMR}\left(377 \mathrm{MHz}, \mathrm{CDCl}_{3}\right) \delta-90.89(\mathrm{~d}, J=259.9 \mathrm{~Hz}$, 1F), $-92.86(\mathrm{~d}, J=259.9 \mathrm{~Hz}, 1 \mathrm{~F})$; ESI-MS m/z $437.1(\mathrm{M}+\mathrm{H})^{+}$; HRMS (ESI) calcd. for $\mathrm{C}_{24} \mathrm{H}_{17} \mathrm{~N}_{1} \mathrm{Br}_{1} \mathrm{~F}_{2}[\mathrm{M}+\mathrm{H}]^{+} 436.05028$, found:436.05070.

\section{3,3-Difluoro-1-phenyl-9-(thiophen-3-yl)-2,3-dihydro-1 $H$-cyclopenta[b]quinoline} (7I):

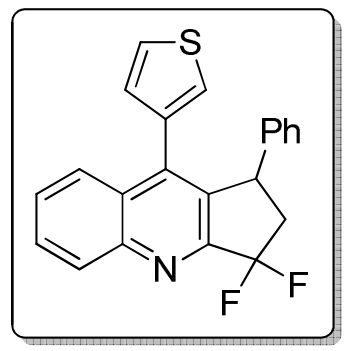

White solid (55 mg, 76\% yield); ${ }^{1} \mathrm{H}$ NMR (400 MHz, $\left.\mathrm{CDCl}_{3}\right) \delta$ $8.34(\mathrm{~d}, J=8.5 \mathrm{~Hz}, 1 \mathrm{H}), 7.76(\mathrm{dd}, J=8.2,7.1 \mathrm{~Hz}, 1 \mathrm{H}), 7.70(\mathrm{~d}$, $J=8.4 \mathrm{~Hz}, 1 \mathrm{H}), 7.56-7.38(\mathrm{~m}, 1 \mathrm{H}), 7.26(\mathrm{~s}, 1 \mathrm{H}), 7.10(\mathrm{dd}, J$ $=6.6,3.6 \mathrm{~Hz}, 3 \mathrm{H}), 6.96-6.68(\mathrm{~m}, 4 \mathrm{H}), 4.69-4.43(\mathrm{~m}, 1 \mathrm{H})$, $3.22(\mathrm{ddd}, J=29.6,15.2,9.2 \mathrm{~Hz}, 1 \mathrm{H}), 2.72(\mathrm{ddd}, J=27.2$, 15.3, $4.4 \mathrm{~Hz}, 1 \mathrm{H}) ;{ }^{13} \mathrm{C} \mathrm{NMR}\left(101 \mathrm{MHz}, \mathrm{CDCl}_{3}\right) \delta 155.53(\mathrm{t}, J=24.5 \mathrm{~Hz}), 149.40$, 
$143.51,142.84,135.46,134.68,130.61,129.98,129.39$, 128.56, 127.85, 127.60, 126.73, $125.97(\mathrm{t}, J=247.3 \mathrm{~Hz}), 125.09,44.42(\mathrm{t}, J=23.2 \mathrm{~Hz}), 43.45(\mathrm{~m}) ;{ }^{19} \mathrm{~F}$ NMR $\left(377 \mathrm{MHz}, \mathrm{CDCl}_{3}\right) \delta-89.49(\mathrm{~d}, J=263.6 \mathrm{~Hz}, 1 \mathrm{~F}),-93.79(\mathrm{~d}, J=252.9 \mathrm{~Hz}, 1 \mathrm{~F})$; EI-MS (m/z, relative intensity): 363 (M+1 100), 330 (28), 281 (18), 207 (63); HRMS (EI) calcd. for $\mathrm{C}_{22} \mathrm{H}_{15} \mathrm{~N}_{1} \mathrm{~F}_{2} \mathrm{~S}_{1}[\mathrm{M}]^{+} 363.0888$, found: 363.0885 .

\section{9-Cyclopropyl-3,3-difluoro-1-phenyl-2,3-dihydro-1H-cyclopenta[b]quinoline} (7m):

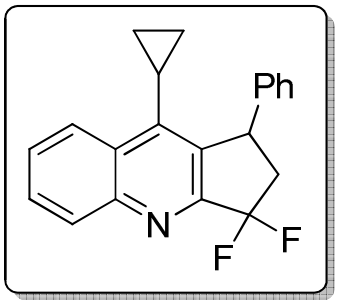

White solid (45 mg, 70\% yield); ${ }^{1} \mathrm{H}$ NMR (400 $\left.\mathrm{MHz}^{\mathrm{CDCl}}{ }_{3}\right) \delta$ $8.44(\mathrm{~d}, J=8.5 \mathrm{~Hz}, 1 \mathrm{H}), 8.29(\mathrm{~d}, J=8.5 \mathrm{~Hz}, 1 \mathrm{H}), 7.75$ (t, $J=$ $7.6 \mathrm{~Hz}, 1 \mathrm{H}), 7.60(\mathrm{t}, J=7.7 \mathrm{~Hz}, 1 \mathrm{H}), 7.34-7.18(\mathrm{~m}, 3 \mathrm{H}), 7.13$ $(\mathrm{d}, J=7.8 \mathrm{~Hz}, 2 \mathrm{H}), 4.81(\mathrm{~s}, 1 \mathrm{H}), 3.50-3.14(\mathrm{~m}, 1 \mathrm{H}), 2.61(\mathrm{qd}$, $J=15.4,5.9 \mathrm{~Hz}, 1 \mathrm{H}), 1.55(\mathrm{dt}, J=14.4,7.3 \mathrm{~Hz}, 1 \mathrm{H}), 1.24-$ $1.09(\mathrm{~m}, 1 \mathrm{H}), 0.75-0.57(\mathrm{~m}, 3 \mathrm{H}) ;{ }^{13} \mathrm{C} \mathrm{NMR}\left(101 \mathrm{MHz}, \mathrm{CDCl}_{3}\right) \delta 155.74(\mathrm{t}, J=24.2$ Hz), 149.29, 146.54, 144.04, 136.31 (t, $J=4.1 \mathrm{~Hz}), 131.00,130.44,129.63,129.14-$ $128.61(\mathrm{~m}), 127.71,127.33,126.95,125.61(\mathrm{dd}, J=248.8,245.2 \mathrm{~Hz}), 125.49,45.11$ $(\mathrm{dd}, J=24.1,22.0 \mathrm{~Hz}), 43.41-43.33(\mathrm{~m}), 11.55,9.06,7.24 ;{ }^{19} \mathrm{~F}$ NMR $(377 \mathrm{MHz}$, $\left.\mathrm{CDCl}_{3}\right) \delta-92.69(\mathrm{~d}, J=258.3 \mathrm{~Hz}, 1 \mathrm{~F}),-94.14(\mathrm{~d}, J=258.3 \mathrm{~Hz}, 1 \mathrm{~F}) ;$ EI-MS (m/z, relative intensity): $321\left(\mathrm{M}^{+}, 100\right), 292$ (72), 272 (33), 243 (60), 207 (66); HRMS (EI) calcd. for $\mathrm{C}_{21} \mathrm{H}_{17} \mathrm{~N}_{1} \mathrm{~F}_{2}[\mathrm{M}]^{+}$321.1324, found: 321,1323.

3,3-Difluoro-1-phenyl-9-(phenylethynyl)-2,3-dihydro-1H-cyclopenta[b]quinoline (7n):

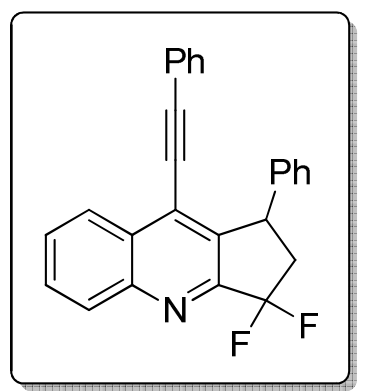

White solid (56 mg, 74\% yield); ${ }^{1} \mathrm{H}$ NMR (400 MHz, $\mathrm{CDCl}_{3}$ ) $\delta 8.36(\mathrm{dd}, J=8.4,0.9 \mathrm{~Hz}, 1 \mathrm{H}), 8.32(\mathrm{~d}, J=8.4 \mathrm{~Hz}, 1 \mathrm{H}), 7.81$ (ddd, $J=8.4,6.9,1.4 \mathrm{~Hz}, 1 \mathrm{H}), 7.68(\mathrm{ddd}, J=8.1,6.9,1.1 \mathrm{~Hz}$, 1H), $7.40-7.30(\mathrm{~m}, 5 \mathrm{H}), 7.29-7.21(\mathrm{~m}, 5 \mathrm{H}), 4.85-4.75(\mathrm{~m}$, 1H), 3.29 (ddt, $J=16.7,15.1,9.3 \mathrm{~Hz}, 1 \mathrm{H}), 2.87-2.62(\mathrm{~m}$,

$1 \mathrm{H}) ;{ }^{13} \mathrm{C} \mathrm{NMR}\left(101 \mathrm{MHz}, \mathrm{CDCl}_{3}\right) \delta 155.66(\mathrm{t}, J=24.8 \mathrm{~Hz}), 148.85,142.39,137.39$ $(\mathrm{t}, J=4.3 \mathrm{~Hz}), 131.96,130.81,130.42,129.67,129.21,128.93,128.48,128.42$, 128.40, 128.17, 127.23, 125.94, 125.64 (dd, $J=248.9,246.3 \mathrm{~Hz}), 121.84,105.72$, 
82.94, $44.84(\mathrm{t}, J=23.6 \mathrm{~Hz}), 44.05(\mathrm{t}, J=3.1 \mathrm{~Hz}) ;{ }^{19} \mathrm{~F} \mathrm{NMR}\left(377 \mathrm{MHz}, \mathrm{CDCl}_{3}\right) \delta$ $-91.68(\mathrm{~d}, J=259.4 \mathrm{~Hz}, 1 \mathrm{~F}),-93.82$ (d, $J=259.4 \mathrm{~Hz}, 1 \mathrm{~F})$; EI-MS (m/z, relative intensity): 381 (M+2 28), 304 (15), 281 (17), 207 (100), 191 (15); HRMS (EI) calcd. for $\mathrm{C}_{26} \mathrm{H}_{17} \mathrm{~N}_{1} \mathrm{~F}_{2}[\mathrm{M}]^{+}$381.1324, found: 381.1325 .

\section{3,3-Difluoro-7-methyl-1,9-diphenyl-2,3-dihydro-1H-cyclopenta[b]quinoline (70):}

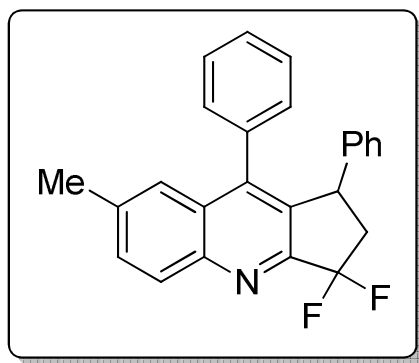

White solid (54 mg, 73\% yield); ${ }^{1} \mathrm{H}$ NMR (400 MHz, $\left.\mathrm{CDCl}_{3}\right) \delta 8.24(\mathrm{~d}, J=8.7 \mathrm{~Hz}, 1 \mathrm{H}), 7.59(\mathrm{dd}, J=8.7,1.8$ $\mathrm{Hz}, 1 \mathrm{H}), 7.44(\mathrm{td}, J=7.5,0.7 \mathrm{~Hz}, 1 \mathrm{H}), 7.35-7.27$ (m, 2H), $7.24(\mathrm{~d}, J=7.6 \mathrm{~Hz}, 1 \mathrm{H}), 7.09-6.92(\mathrm{~m}, 4 \mathrm{H}), 6.69$ $(\mathrm{dd}, J=7.8,1.5 \mathrm{~Hz}, 2 \mathrm{H}), 6.50(\mathrm{~d}, J=7.7 \mathrm{~Hz}, 1 \mathrm{H}), 4.51$

$(\mathrm{dt}, J=9.1,4.5 \mathrm{~Hz}, 1 \mathrm{H}), 3.36-3.00(\mathrm{~m}, 1 \mathrm{H}), 2.79-2.63(\mathrm{~m}, 1 \mathrm{H}), 2.40(\mathrm{~s}, 3 \mathrm{H}) ;{ }^{13} \mathrm{C}$ NMR (101 MHz, $\left.\mathrm{CDCl}_{3}\right) \delta 154.41(\mathrm{t}, J=24.5 \mathrm{~Hz}), 148.07,146.51,143.42,137.96$, $135.12(\mathrm{t}, J=4.2 \mathrm{~Hz}), 134.98,132.32,130.20,129.41,129.07,128.53,128.51$, $128.34,128.06,128.01,127.76,126.49,126.21(\mathrm{t}, J=247.1 \mathrm{~Hz}), 124.67,44.30$ (t, $J$ $=23.3 \mathrm{~Hz}), 43.24(\mathrm{t}, J=3.2 \mathrm{~Hz}), 21.97 ;{ }^{19} \mathrm{~F} \mathrm{NMR}\left(377 \mathrm{MHz}, \mathrm{CDCl}_{3}\right) \delta-88.91(\mathrm{~d}, J=$ $258.8 \mathrm{~Hz}, 1 \mathrm{~F}),-93.21$ (d, $J=258.8 \mathrm{~Hz}, 1 \mathrm{~F})$; EI-MS (m/z, relative intensity): $371\left(\mathrm{M}^{+}\right.$, 100), 356 (22), 294 (87), 274 (27), 207 (54);HRMS (EI) calcd. for $\mathrm{C}_{25} \mathrm{H}_{19} \mathrm{~N}_{1} \mathrm{~F}_{2}[\mathrm{M}]^{+}$ 371.1480, found: 371.1479 .

\section{3,3-Difluoro-7-methoxy-1,9-diphenyl-2,3-dihydro-1H-cyclopenta[b]quinoline}

(7p):

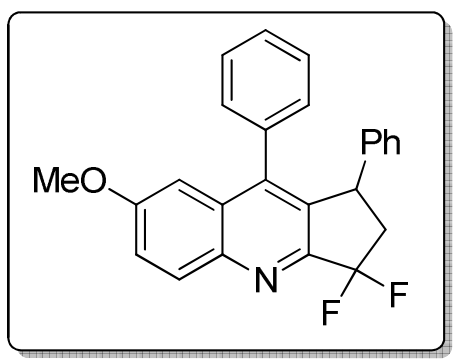

White solid (63 mg, 82\% yield); ${ }^{1} \mathrm{H}$ NMR (400 MHz, $\left.\mathrm{CDCl}_{3}\right) \delta 8.34(\mathrm{~d}, J=8.5 \mathrm{~Hz}, 1 \mathrm{H}), 7.75(\mathrm{ddd}, J=8.4$, 6.8, $1.4 \mathrm{~Hz}, 1 \mathrm{H}), 7.62(\mathrm{dd}, J=8.5,0.8 \mathrm{~Hz}, 1 \mathrm{H}), 7.47$ (ddd, $J=8.2,6.8,1.1 \mathrm{~Hz}, 1 \mathrm{H}), 7.17$ (dd, $J=8.4,2.1 \mathrm{~Hz}$, 1H), $7.11-7.00(\mathrm{~m}, 3 \mathrm{H}), 6.96(\mathrm{dd}, J=8.4,2.6 \mathrm{~Hz}, 1 \mathrm{H})$, $6.77-6.68(\mathrm{~m}, 2 \mathrm{H}), 6.59(\mathrm{dd}, J=8.5,2.6 \mathrm{~Hz}, 1 \mathrm{H}), 6.45(\mathrm{dd}, J=8.5,2.1 \mathrm{~Hz}, 1 \mathrm{H})$, $4.55(\mathrm{dt}, J=9.0,4.4 \mathrm{~Hz}, 1 \mathrm{H}), 3.82$ (s, 3H), 3.21 (dddd, $J=17.2,15.3,12.5,9.2 \mathrm{~Hz}$, 1H), $2.94-2.63(\mathrm{~m}, 1 \mathrm{H}) ;{ }^{13} \mathrm{C} \mathrm{NMR}\left(101 \mathrm{MHz}, \mathrm{CDCl}_{3}\right) \delta 158.82,152.97(\mathrm{t}, J=24.5$ 
Hz), 145.73, 145.61, 143.45, 135.49 (t, $J=4.0 \mathrm{~Hz}), 135.10,131.95,130.41,129.22$, $128.68,128.52,128.35,128.18,128.07,127.78,126.49,126.30(\mathrm{t}, J=246.7 \mathrm{~Hz})$, $122.75,103.64,55.49,44.38(\mathrm{t}, J=23.4 \mathrm{~Hz}), 43.27 ;{ }^{19} \mathrm{~F}$ NMR $\left(377 \mathrm{MHz}, \mathrm{CDCl}_{3}\right) \delta$ $-89.22(\mathrm{~d}, J=259.2 \mathrm{~Hz}, 1 \mathrm{~F}),-93.53(\mathrm{~d}, J=259.4 \mathrm{~Hz}, 1 \mathrm{~F})$; EI-MS (m/z, relative intensity): $387\left(\mathrm{M}^{+}, 60\right), 310$ (15), 341 (7), 281 (24),207 (100), 191(14); HRMS (EI) calcd. for $\mathrm{C}_{25} \mathrm{H}_{19} \mathrm{O}_{1} \mathrm{~N}_{1} \mathrm{~F}_{2}[\mathrm{M}]^{+}$387.1429, found: 387.1426 .

\section{7-Bromo-3,3-difluoro-1,9-diphenyl-2,3-dihydro-1H-cyclopenta[b]quinoline (7q):}

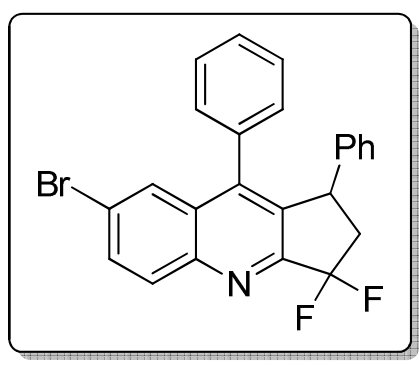

White solid (70 mg, 81\% yield); ${ }^{1} \mathrm{H}$ NMR (400 MHz, $\left.\mathrm{CDCl}_{3}\right) \delta 8.20(\mathrm{~d}, J=9.0 \mathrm{~Hz}, 1 \mathrm{H}), 7.82(\mathrm{dd}, J=9.0,1.9$ $\mathrm{Hz}, 1 \mathrm{H}), 7.70(\mathrm{~d}, J=2.1 \mathrm{~Hz}, 1 \mathrm{H}), 7.45(\mathrm{td}, J=7.5,0.7 \mathrm{~Hz}$, 1H), 7.32 (tt, $J=7.5,1.2 \mathrm{~Hz}, 1 \mathrm{H}), 7.27-7.20(\mathrm{~m}, 1 \mathrm{H})$, $7.11-6.94(\mathrm{~m}, 4 \mathrm{H}), 6.68(\mathrm{dd}, J=7.9,1.4 \mathrm{~Hz}, 2 \mathrm{H}), 6.49$ $(\mathrm{d}, J=7.7 \mathrm{~Hz}, 1 \mathrm{H}), 4.53(\mathrm{dt}, J=9.0,4.4 \mathrm{~Hz}, 1 \mathrm{H}), 3.48-3.00(\mathrm{~m}, 1 \mathrm{H}), 2.93-2.59$ $(\mathrm{m}, 1 \mathrm{H}) ;{ }^{13} \mathrm{C} \mathrm{NMR}\left(101 \mathrm{MHz}, \mathrm{CDCl}_{3}\right) \delta 155.84(\mathrm{t}, J=24.6 \mathrm{~Hz}), 148.04,146.57$, 142.93, 136.02 (t, $J=3.6 \mathrm{~Hz}$ ), 134.07, 133.51, 132.17, 130.26, 129.30, 128.72, 128.46, 128.34, 128.17, 127.75, 126.70, $125.87(\mathrm{t}, J=247.3 \mathrm{~Hz}), 122.35,44.22(\mathrm{t}, J$ $=23.2 \mathrm{~Hz}), 43.30 ;{ }^{19} \mathrm{~F}$ NMR $\left(377 \mathrm{MHz}, \mathrm{CDCl}_{3}\right) \delta-89.49(\mathrm{~d}, J=260.4 \mathrm{~Hz}, 1 \mathrm{~F})$, -93.59 (d, $J=260.5 \mathrm{~Hz}, 1 \mathrm{~F})$; EI-MS (m/z, relative intensity): $437\left({ }^{81} \mathrm{Br}, \mathrm{M}^{+}, 95\right), 435$ $\left({ }^{79} \mathrm{Br}, \mathrm{M}^{+}, 100\right), 360$ (36), 356 (77), 336 (30), 258 (45), 207 (100); HRMS (EI) calcd. for $\mathrm{C}_{24} \mathrm{H}_{16} \mathrm{~N}_{1} \mathrm{Br}_{1} \mathrm{~F}_{2}[\mathrm{M}]^{+} 435.0429$, found:435.0426.

\section{3,3-Difluoro-5-methyl-1,9-diphenyl-2,3-dihydro-1H-cyclopenta[b]quinoline (7r):}

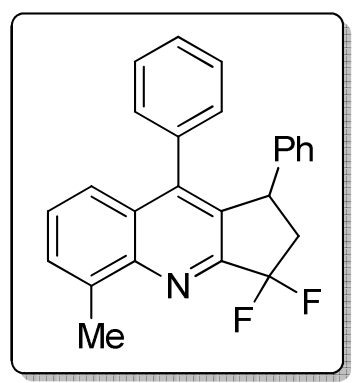

White solid (66 mg, 89\% yield); ${ }^{1} \mathrm{H}$ NMR (400 MHz, $\mathrm{CDCl}_{3}$ ) $\delta 7.60(\mathrm{~d}, J=6.6 \mathrm{~Hz}, 1 \mathrm{H}), 7.45-7.32(\mathrm{~m}, 3 \mathrm{H}), 7.29(\mathrm{tt}, J=$ 7.5, $1.2 \mathrm{~Hz}, 1 \mathrm{H}), 7.24(\mathrm{~d}, J=7.6 \mathrm{~Hz}, 1 \mathrm{H}), 7.07-6.98(\mathrm{~m}, 4 \mathrm{H})$, $6.73-6.67(\mathrm{~m}, 2 \mathrm{H}), 6.51(\mathrm{~d}, J=7.7 \mathrm{~Hz}, 1 \mathrm{H}), 4.53(\mathrm{dt}, J=9.1$, $4.5 \mathrm{~Hz}, 1 \mathrm{H}), 3.32-3.13(\mathrm{~m}, 1 \mathrm{H}), 2.97(\mathrm{~s}, 3 \mathrm{H}), 2.85-2.63(\mathrm{~m}$, $1 \mathrm{H}) ;{ }^{13} \mathrm{C} \mathrm{NMR}\left(101 \mathrm{MHz}, \mathrm{CDCl}_{3}\right) \delta 154.07$ (t, $\left.J=24.3 \mathrm{~Hz}\right), 148.71,147.19,143.51$ (d, $J=1.9 \mathrm{~Hz}), 138.61,135.29$ (s), 134.71 (t, $J=4.3 \mathrm{~Hz}), 129.87,129.48,129.03$, 
$128.55,128.43,128.33,127.98,127.94,127.79,127.38,126.47,126.34$ (dd, $J=$ 247.6, 246.5 Hz), 124.01, 124.01, 44.40 (t, $J=23.4 \mathrm{~Hz}), 43.16$ (t, $J=3.3 \mathrm{~Hz}), 18.61$;

${ }^{19} \mathrm{~F}$ NMR $\left(377 \mathrm{MHz}, \mathrm{CDCl}_{3}\right) \delta-88.86(\mathrm{~d}, J=258.5 \mathrm{~Hz}, 1 \mathrm{~F}),-93.10(\mathrm{~d}, J=258.4 \mathrm{~Hz}$, 1F); EI-MS (m/z, relative intensity): 371(M+ 65), 350 (10), 294 (8), 207 (100); HRMS (EI) calcd. for $\mathrm{C}_{25} \mathrm{H}_{19} \mathrm{~N}_{1} \mathrm{~F}_{2}[\mathrm{M}]^{+} 371.1480$, found: 371.1478 .

\section{3,3-Difluoro-8-methyl-1,9-diphenyl-2,3-dihydro-1H-cyclopenta[b]quinoline (7s) and}

\section{3,3-difluoro-6-methyl-1,9-diphenyl-2,3-dihydro-1H-cyclopenta[b]quinoline(7s'):}

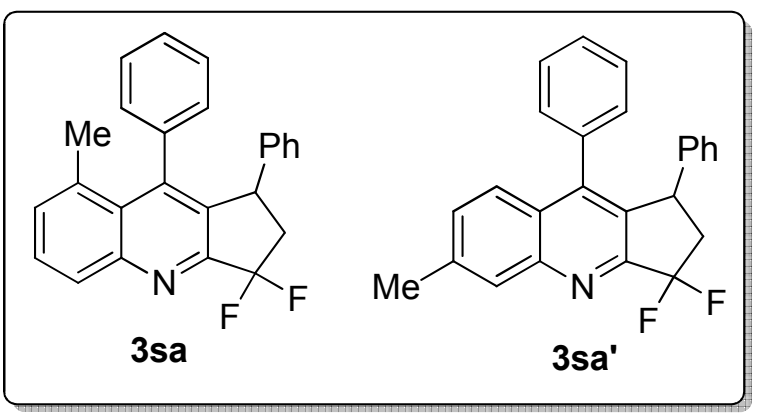

White solid (60 mg, 81\% yield); ${ }^{1} \mathrm{H}$ NMR (400 MHz, $\left.\mathrm{CDCl}_{3}\right) \delta 8.25(\mathrm{~d}, J$ $=8.4 \mathrm{~Hz}, 1 \mathrm{H}), 8.12\left(\mathrm{~s}, 1 \mathrm{H}^{\prime}\right), 7.62(\mathrm{dd}$, $\left.J=8.4,7.1 \mathrm{~Hz}, 3 \mathrm{H}^{\prime}\right), 7.47-7.37(\mathrm{~m}$, $\left.6 \mathrm{H}^{\prime}\right), 7.33-7.21(\mathrm{~m}, 4 \mathrm{H}), 7.12-6.95$ $(\mathrm{m}, 4 \mathrm{H}), 6.86(\mathrm{t}, J=7.6 \mathrm{~Hz}, 1 \mathrm{H}), 6.70$ $\left(\mathrm{dd}, J=7.8,1.6 \mathrm{~Hz}, 2 \mathrm{H}^{\prime}\right), 6.63-6.58(\mathrm{~m}, 2 \mathrm{H}), 6.51\left(\mathrm{~d}, J=7.7 \mathrm{~Hz}, 1 \mathrm{H}^{\prime}\right), 6.32(\mathrm{~d}, J=$ $7.7 \mathrm{~Hz}, 1 \mathrm{H}), 4.52\left(\mathrm{dt}, J=9.0,4.5 \mathrm{~Hz}, 1 \mathrm{H}^{\prime}\right), 4.40-4.25(\mathrm{~m}, 1 \mathrm{H}), 3.29-3.19\left(\mathrm{~m}, 1 \mathrm{H}^{\prime}\right)$, 3.14 (ddt, $J=17.4,15.5,6.3 \mathrm{~Hz}, 1 \mathrm{H}), 2.73\left(\mathrm{ddd}, J=7.9,6.6,3.7 \mathrm{~Hz}, 1 \mathrm{H}^{\prime}\right), 2.71-$ $2.61(\mathrm{~m}, 1 \mathrm{H}), 2.57\left(\mathrm{~s}, 3 \mathrm{H}^{\prime}\right), 1.91(\mathrm{~s}, 3 \mathrm{H}) ;{ }^{13} \mathrm{C} \mathrm{NMR}\left(101 \mathrm{MHz}, \mathrm{CDCl}_{3}\right) \delta 155.29(\mathrm{t}, J$ $=24.3 \mathrm{~Hz}), 154.34(\mathrm{t}, J=24.6 \mathrm{~Hz}), 151.03,149.75,147.83,147.09,144.17,143.48$, 140.37, 138.57, 136.32, 136.24, 134.99, 134.29 (t, $J=7.4 \mathrm{~Hz}), 131.25,130.10$, $129.97,129.55,129.48,129.42,128.55,128.48,128.38,128.35,128.05,127.86$, 127.77, 127.72, 127.63, 127.56, 127.18, 126.49, 126.45, 126.24 (t, $J=247.1 \mathrm{~Hz})$, $126.17(\mathrm{t}, J=247.1 \mathrm{~Hz}), 125.70,44.37(\mathrm{t}, J=22.8 \mathrm{~Hz}), 44.15(\mathrm{t}, J=20.4 \mathrm{~Hz}), 43.62$ $(\mathrm{d}, J=3.2 \mathrm{~Hz}), 43.19,24.68,21.81 ;{ }^{19} \mathrm{~F}$ NMR $\left(377 \mathrm{MHz}, \mathrm{CDCl}_{3}\right) \delta-86.59(\mathrm{~d}, J=$ 259.4 Hz, 1F), -89.15 (d, $\left.J=259.1 \mathrm{~Hz}, 1 \mathrm{~F}^{\prime}\right),-93.35$ (d, $\left.J=259.2 \mathrm{~Hz}, 1 \mathrm{~F}^{\prime}\right),-94.43$ (d, $J=259.4 \mathrm{~Hz}, 1 \mathrm{~F})$; EI-MS (m/z, relative intensity): $371\left(\mathrm{M}^{+}, 100\right), 351$ (15), 294 (50), 274 (18), 207 (30); HRMS (EI) calcd. for $\mathrm{C}_{25} \mathrm{H}_{19} \mathrm{~N}_{1} \mathrm{~F}_{2}[\mathrm{M}]^{+}$371.1480, found: 371.1480 . 


\section{3,3-Difluoro-9-phenyl-1-(p-tolyl)-2,3-dihydro-1H-cyclopenta[b]quinoline (9a):}

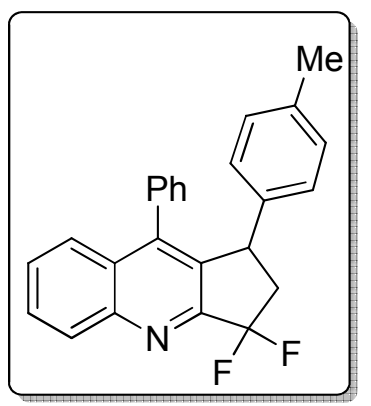

White solid (64 mg, 87\% yield); ${ }^{1} \mathrm{H}$ NMR (400 MHz, $\mathrm{CDCl}_{3}$ ) $\delta 8.39(\mathrm{~d}, J=8.5 \mathrm{~Hz}, 1 \mathrm{H}), 7.78(\mathrm{ddd}, J=8.4,6.7,1.4 \mathrm{~Hz}$, 1H), $7.60(\mathrm{dd}, J=8.5,0.9 \mathrm{~Hz}, 1 \mathrm{H}), 7.53-7.43(\mathrm{~m}, 2 \mathrm{H}), 7.34$ $(\mathrm{tt}, J=7.5,1.2 \mathrm{~Hz}, 1 \mathrm{H}), 7.30-7.25(\mathrm{~m}, 1 \mathrm{H}), 7.10(\mathrm{td}, J=7.6$, $0.8 \mathrm{~Hz}, 1 \mathrm{H}), 6.84(\mathrm{~d}, J=7.9 \mathrm{~Hz}, 2 \mathrm{H}), 6.59$ (dd, $J=15.1,7.9$ $\mathrm{Hz}, 3 \mathrm{H}), 4.54(\mathrm{dt}, J=8.9,4.4 \mathrm{~Hz}, 1 \mathrm{H}), 3.22(\mathrm{dddd}, J=17.3$, 15.3, 12.7, 9.2 Hz, 1H), 2.74 (dddd, $J=16.4,15.2,10.6,4.4 \mathrm{~Hz}, 1 \mathrm{H}), 2.25$ (s, 3H); ${ }^{13} \mathrm{C}$ NMR (101 MHz, $\left.\mathrm{CDCl}_{3}\right) \delta 155.29(\mathrm{t}, J=24.5 \mathrm{~Hz}), 149.22,147.45,140.24$ (d, $J$ $=1.5 \mathrm{~Hz}), 136.08,135.22(\mathrm{t}, J=4.0 \mathrm{~Hz}), 134.77,130.40,129.91,129.41,129.08$, 128.99, 128.47, 128.11, 127.99, 127.73, 127.59, 126.08 (t, $J=247.9 \mathrm{~Hz}), 44.33$ (t, $J$ $=23.0 \mathrm{~Hz}), 42.83(\mathrm{t}, J=3.2 \mathrm{~Hz}), 21.04 ;{ }^{19} \mathrm{~F} \mathrm{NMR}\left(377 \mathrm{MHz}, \mathrm{CDCl}_{3}\right) \delta-89.04(\mathrm{~d}, J=$ $259.5 \mathrm{~Hz}, 1 \mathrm{~F}),-93.65$ (d, $J=259.5 \mathrm{~Hz}, 1 \mathrm{~F})$; EI-MS (m/z, relative intensity): $371\left(\mathrm{M}^{+}\right.$, 100), 356 (41), 294 (55), 185 (13); HRMS (EI) calcd. for $\mathrm{C}_{25} \mathrm{H}_{19} \mathrm{~N}_{1} \mathrm{~F}_{2}[\mathrm{M}]^{+}$371.1480, found: 371.1483 .

\section{3,3-Difluoro-9-phenyl-1-(m-tolyl)-2,3-dihydro-1H-cyclopenta[b]quinoline (9b):}

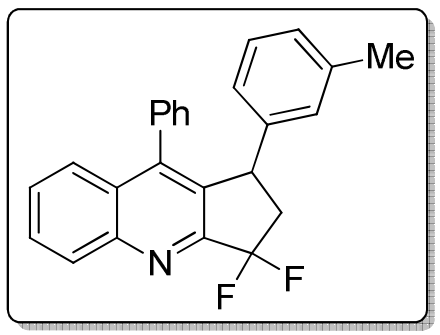

White solid (67 mg, 90\% yield); ${ }^{1} \mathrm{H}$ NMR (400 MHz, $\left.\mathrm{CDCl}_{3}\right) \delta 8.37(\mathrm{~d}, J=8.5 \mathrm{~Hz}, 1 \mathrm{H}), 7.81-7.74(\mathrm{~m}, 1 \mathrm{H})$, $7.59(\mathrm{~d}, J=8.4 \mathrm{~Hz}, 1 \mathrm{H}), 7.53-7.43(\mathrm{~m}, 2 \mathrm{H}), 7.33(\mathrm{td}, J=$ 7.5, $1.1 \mathrm{~Hz}, 1 \mathrm{H}), 7.28(\mathrm{~s}, 1 \mathrm{H}), 7.08(\mathrm{t}, J=7.6 \mathrm{~Hz}, 1 \mathrm{H})$, $6.93(\mathrm{t}, J=7.5 \mathrm{~Hz}, 1 \mathrm{H}), 6.86(\mathrm{~d}, J=7.5 \mathrm{~Hz}, 1 \mathrm{H}), 6.54(\mathrm{t}$, $J=6.8 \mathrm{~Hz}, 2 \mathrm{H}), 6.47(\mathrm{~s}, 1 \mathrm{H}), 4.52(\mathrm{dt}, J=9.1,4.5 \mathrm{~Hz}, 1 \mathrm{H}), 3.32-3.14(\mathrm{~m}, 1 \mathrm{H}), 2.77$ $(\operatorname{tdd}, J=16.3,11.5,4.7 \mathrm{~Hz}, 1 \mathrm{H}), 2.13(\mathrm{~s}, 3 \mathrm{H}) ;{ }^{13} \mathrm{C} \mathrm{NMR}\left(101 \mathrm{MHz}, \mathrm{CDCl}_{3}\right) \delta 155.32$ (t, $J=24.5 \mathrm{~Hz}), 149.35,147.33,143.03,137.81,135.23$ (t, $J=4.1 \mathrm{~Hz}), 134.80$, 130.52 , 129.87, 129.42, 129.07, 128.82, 128.56, 128.46, 128.30, 128.03, 127.93, 127.71, 127.24, 126.10 (dd, $J=245.3,243.4 \mathrm{~Hz}), 126.06,124.67,44.14$ (t, $J=23.2$ $\mathrm{Hz}), 43.17(\mathrm{t}, J=3.2 \mathrm{~Hz}), 21.24 ;{ }^{19} \mathrm{~F} \mathrm{NMR}\left(377 \mathrm{MHz}, \mathrm{CDCl}_{3}\right) \delta-89.49(\mathrm{~d}, J=259.5$ $\mathrm{Hz}, 1 \mathrm{~F}),-93.12(\mathrm{~d}, J=259.5 \mathrm{~Hz}, 1 \mathrm{~F})$; EI-MS (m/z, relative intensity): $371\left(\mathrm{M}^{+}, 100\right)$, 356 (37), 294 (67), 280 (16); HRMS (EI) calcd. for $\mathrm{C}_{25} \mathrm{H}_{19} \mathrm{~N}_{1} \mathrm{~F}_{2}[\mathrm{M}]^{+}$371.1480, found: 


\section{3,3-Difluoro-9-phenyl-1-(o-tolyl)-2,3-dihydro-1H-cyclopenta[b]quinoline (9c):}

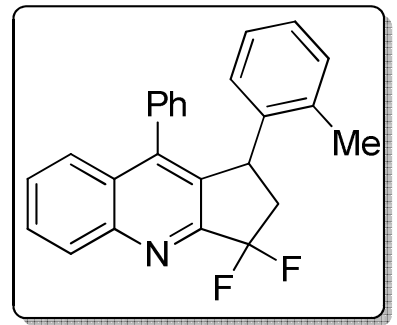

White solid (59 mg, 80\% yield); ${ }^{1} \mathrm{H}$ NMR (400 MHz, $\left.\mathrm{CDCl}_{3}\right) \delta 8.36(\mathrm{~d}, J=8.5 \mathrm{~Hz}, 1 \mathrm{H}), 7.76(\mathrm{ddd}, J=8.4,6.7$, $1.5 \mathrm{~Hz}, 1 \mathrm{H}), 7.56(\mathrm{dd}, J=8.4,0.9 \mathrm{~Hz}, 1 \mathrm{H}), 7.51-7.45(\mathrm{~m}$, 1H), $7.38(\mathrm{t}, J=7.5 \mathrm{~Hz}, 1 \mathrm{H}), 7.29-7.24(\mathrm{~m}, 1 \mathrm{H}), 7.21(\mathrm{~d}, J$ $=7.5 \mathrm{~Hz}, 1 \mathrm{H}), 7.03(\mathrm{t}, J=7.5 \mathrm{~Hz}, 1 \mathrm{H}), 6.95(\mathrm{dt}, J=8.8$, $4.2 \mathrm{~Hz}, 2 \mathrm{H}), 6.86(\mathrm{~d}, J=8.6 \mathrm{~Hz}, 1 \mathrm{H}), 6.67-6.61(\mathrm{~m}, 1 \mathrm{H}), 6.53(\mathrm{~d}, J=7.5 \mathrm{~Hz}, 1 \mathrm{H})$, $4.76(\mathrm{dt}, J=8.9,4.4 \mathrm{~Hz}, 1 \mathrm{H}), 3.20(\mathrm{dddd}, J=17.3,15.1,11.4,9.2 \mathrm{~Hz}, 1 \mathrm{H}), 2.60$ (d, $J$ $=14.4 \mathrm{~Hz}, 1 \mathrm{H}), 1.98(\mathrm{~s}, 3 \mathrm{H}) ;{ }^{13} \mathrm{C} \mathrm{NMR}\left(101 \mathrm{MHz}, \mathrm{CDCl}_{3}\right) \delta 155.72(\mathrm{t}, J=24.5 \mathrm{~Hz})$, $149.36,147.07,142.28,135.47,135.20,134.84,130.55,129.86,129.10(\mathrm{~d}, J=5.1$ $\mathrm{Hz}), 128.38,128.29,128.10,127.97,127.74,127.28,126.63,126.47,126.12$ (t, $J=$ 247.3 Hz), 126.12, $43.77(\mathrm{t}, J=21.7 \mathrm{~Hz}), 38.20,19.53 ;{ }^{19} \mathrm{~F} \mathrm{NMR}\left(377 \mathrm{MHz}, \mathrm{CDCl}_{3}\right)$ $\delta-89.19(\mathrm{~d}, J=259.3 \mathrm{~Hz}, 1 \mathrm{~F}),-93.35(\mathrm{~d}, J=259.4 \mathrm{~Hz}, 1 \mathrm{~F})$; EI-MS (m/z, relative intensity): $371\left(\mathrm{M}^{+}, 100\right), 356$ (34), 294 (66), 280 (16); HRMS (EI) calcd. for $\mathrm{C}_{25} \mathrm{H}_{19} \mathrm{~N}_{1} \mathrm{~F}_{2}[\mathrm{M}]^{+}$371.1480, found: 371.1478 .

\section{3,3-Difluoro-1-(4-methoxyphenyl)-9-phenyl-2,3-dihydro-1 $H$-cyclopenta[b]quinoli} ne (9d):

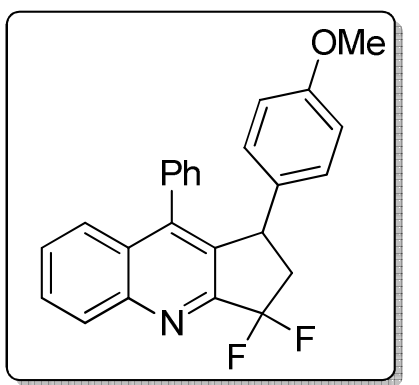

White solid (69 mg, 90\% yield); ${ }^{1} \mathrm{H}$ NMR (400 MHz, $\left.\mathrm{CDCl}_{3}\right) \delta 8.35(\mathrm{~d}, J=8.5 \mathrm{~Hz}, 1 \mathrm{H}), 7.75(\mathrm{~d}, J=1.5 \mathrm{~Hz}, 1 \mathrm{H})$, $7.56(\mathrm{~d}, J=7.7 \mathrm{~Hz}, 1 \mathrm{H}), 7.50-7.40(\mathrm{~m}, 2 \mathrm{H}), 7.32(\mathrm{~s}, 1 \mathrm{H})$, $7.26(\mathrm{~d}, J=2.0 \mathrm{~Hz}, 1 \mathrm{H}), 7.10(\mathrm{~s}, 1 \mathrm{H}), 6.63-6.58(\mathrm{~m}, 2 \mathrm{H})$, $6.57-6.52(\mathrm{~m}, 3 \mathrm{H}), 4.50(\mathrm{dt}, J=8.9,4.4 \mathrm{~Hz}, 1 \mathrm{H}), 3.71(\mathrm{~s}$, 3H), 3.19 (dddd, $J=17.3,15.3,12.7,9.2 \mathrm{~Hz}, 1 \mathrm{H}), 2.70$ (dddd, $J=19.7,15.2,10.6,4.4 \mathrm{~Hz}, 1 \mathrm{H}) ;{ }^{13} \mathrm{C} \mathrm{NMR}\left(101 \mathrm{MHz}, \mathrm{CDCl}_{3}\right) \delta 158.18$, $155.21(\mathrm{t}, J=24.5 \mathrm{~Hz}), 149.22,147.44,135.47(\mathrm{~d}, J=1.7 \mathrm{~Hz}), 135.32(\mathrm{t}, J=4.1 \mathrm{~Hz})$, $134.80,130.40,129.93,129.38,129.10,128.74,128.52,128.16,128.07,127.75$, $126.06(\mathrm{t}, J=245.1 \mathrm{~Hz}), 113.76,55.36,44.31(\mathrm{t}, J=23.0 \mathrm{~Hz}), 42.44(\mathrm{t}, J=3.2 \mathrm{~Hz})$; 
${ }^{19} \mathrm{~F} \mathrm{NMR}\left(377 \mathrm{MHz}, \mathrm{CDCl}_{3}\right) \delta-88.84(\mathrm{~d}, J=259.6,1 \mathrm{~F}),-93.89(\mathrm{~d}, J=259.6 \mathrm{~Hz}, 1 \mathrm{~F})$; EI-MS (m/z, relative intensity): $387\left(\mathrm{M}^{+}, 74\right), 346$ (25), 280 (18), 207 (100); HRMS (EI) calcd. for $\mathrm{C}_{25} \mathrm{H}_{19} \mathrm{O}_{1} \mathrm{~N}_{1} \mathrm{~F}_{2}[\mathrm{M}]^{+}$387.1429, found: 387.1427 .

\section{3,3-Difluoro-1-(4-fluorophenyl)-9-phenyl-2,3-dihydro-1 $H$-cyclopenta[b]quinoline} (9e):

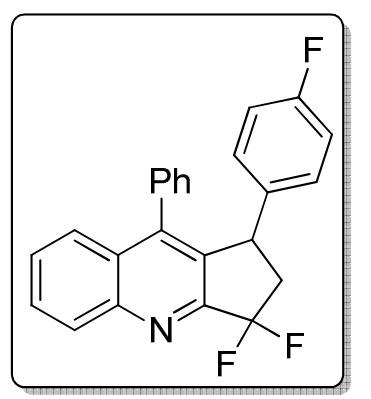

White solid (70 mg, 93\% yield); ${ }^{1} \mathrm{H}$ NMR (400 $\mathrm{MHz}, \mathrm{CDCl}_{3}$ ) $\delta 8.35(\mathrm{~d}, J=8.5 \mathrm{~Hz}, 1 \mathrm{H}), 7.76(\mathrm{ddd}, J=8.4,6.7,1.4 \mathrm{~Hz}$, 1H), $7.57(\mathrm{dd}, J=8.4,0.9 \mathrm{~Hz}, 1 \mathrm{H}), 7.51-7.41(\mathrm{~m}, 2 \mathrm{H})$, $7.36-7.29(\mathrm{~m}, 1 \mathrm{H}), 7.25(\mathrm{~d}, J=6.5 \mathrm{~Hz}, 1 \mathrm{H}), 7.11(\mathrm{td}, J=$ 7.6, $0.8 \mathrm{~Hz}, 1 \mathrm{H}), 6.73-6.62(\mathrm{~m}, 4 \mathrm{H}), 6.55(\mathrm{~d}, J=7.7 \mathrm{~Hz}$, $1 \mathrm{H}), 4.55(\mathrm{dt}, J=9.0,4.4 \mathrm{~Hz}, 1 \mathrm{H}), 3.21$ (dddd, $J=17.1$, 15.4, 12.2, 9.2 Hz, 1H), $2.75-2.60(\mathrm{~m}, 1 \mathrm{H}) ;{ }^{13} \mathrm{C} \mathrm{NMR}\left(101 \mathrm{MHz}, \mathrm{CDCl}_{3}\right) \delta 161.46$ $(\mathrm{d}, J=245.2 \mathrm{~Hz}), 155.13(\mathrm{t}, J=24.5 \mathrm{~Hz}), 149.40,147.38,138.95(\mathrm{~d}, J=2.1 \mathrm{~Hz})$, $134.84(\mathrm{t}, J=4.1 \mathrm{~Hz}), 134.75,130.51,130.07,129.29,129.21,129.01,128.64$, $128.48,128.28,128.16,127.88,126.03,125.94(\mathrm{t}, J=247.2 \mathrm{~Hz}), 115.16(\mathrm{~d}, J=21.4$ $\mathrm{Hz}), 44.24(\mathrm{t}, J=23.3 \mathrm{~Hz}), 42.48(\mathrm{t}, J=3.2 \mathrm{~Hz}) ;{ }^{19} \mathrm{~F}$ NMR $\left(377 \mathrm{MHz}, \mathrm{CDCl}_{3}\right) \delta$ $-89.00(\mathrm{~d}, J=260.0 \mathrm{~Hz}, 1 \mathrm{~F}),-93.81$ (d, $J=259.9 \mathrm{~Hz}, 1 \mathrm{~F}),-116.16$ (s, 1F); EI-MS (m/z, relative intensity): $375\left(\mathrm{M}^{+}, 100\right), 355$ (17), 298 (74), 278 (24), 207 (35); HRMS (EI) calcd. for $\mathrm{C}_{24} \mathrm{H}_{16} \mathrm{~N}_{1} \mathrm{~F}_{3}[\mathrm{M}]^{+} 375.1229$, found: 375.1228 .

\section{1-(4-Chlorophenyl)-3,3-difluoro-9-phenyl-2,3-dihydro-1H-cyclopenta[b]quinoline} (9f):

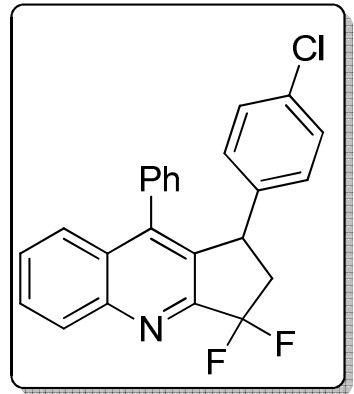

White solid (67 mg, 86\% yield); ${ }^{1} \mathrm{H} \mathrm{NMR}\left(400 \mathrm{MHz}, \mathrm{CDCl}_{3}\right)$ $\delta 8.35(\mathrm{~d}, J=8.5 \mathrm{~Hz}, 1 \mathrm{H}), 7.77(\mathrm{ddd}, J=8.4,6.7,1.4 \mathrm{~Hz}, 1 \mathrm{H})$, $7.58(\mathrm{dd}, J=8.4,0.9 \mathrm{~Hz}, 1 \mathrm{H}), 7.51-7.41(\mathrm{~m}, 2 \mathrm{H}), 7.37-$ $7.30(\mathrm{~m}, 1 \mathrm{H}), 7.25(\mathrm{~d}, J=8.1 \mathrm{~Hz}, 2 \mathrm{H}), 7.13(\mathrm{td}, J=7.6,0.8$ $\mathrm{Hz}, 1 \mathrm{H}), 7.01-6.93(\mathrm{~m}, 2 \mathrm{H}), 6.67-6.59(\mathrm{~m}, 2 \mathrm{H}), 6.56(\mathrm{~d}, J$ $=7.7 \mathrm{~Hz}, 1 \mathrm{H}), 4.54(\mathrm{dt}, J=9.0,4.4 \mathrm{~Hz}, 1 \mathrm{H}), 3.21(\mathrm{dddd}, J=$ 16.9, 15.3, 12.3, 9.2 Hz, 1H), $2.68(\operatorname{tdd}, J=15.4,10.8,4.5 \mathrm{~Hz}, 1 \mathrm{H}) ;{ }^{13} \mathrm{C}$ NMR $(101$ 
$\left.\mathrm{MHz}, \mathrm{CDCl}_{3}\right) \delta 155.16(\mathrm{t}, J=24.5 \mathrm{~Hz}), 149.47,147.41,141.70,134.74,134.55(\mathrm{t}, J$ $=3.7 \mathrm{~Hz}), 132.42,130.33(\mathrm{~d}, J=43.5 \mathrm{~Hz}), 129.35,129.09,128.99,128.67,128.48$, 128.35, 128.22, 127.92, 126.05, $124.66(\mathrm{t}, J=247.3 \mathrm{~Hz}), 44.14(\mathrm{t}, J=23.5 \mathrm{~Hz})$, 42.63; ${ }^{19} \mathrm{~F}$ NMR $\left(377 \mathrm{MHz}, \mathrm{CDCl}_{3}\right) \delta-89.00(\mathrm{~d}, J=260.0 \mathrm{~Hz}, 1 \mathrm{~F}),-93.81(\mathrm{~d}, J=$ $260.0 \mathrm{~Hz}, 1 \mathrm{~F})$; EI-MS (m/z, relative intensity): $393\left({ }^{37} \mathrm{Cl}, \mathrm{M}^{+}, 3\right), 391\left({ }^{35} \mathrm{Cl}, \mathrm{M}^{+}, 8\right)$, 356 (11), 306 (100), 289 (11); HRMS (EI) calcd. for $\mathrm{C}_{24} \mathrm{H}_{16} \mathrm{~N}_{1} \mathrm{Cl}_{1} \mathrm{~F}_{2}[\mathrm{M}]^{+} 391.0934$, found: 391.0931 .

\section{1-(4-Bromophenyl)-3,3-difluoro-9-phenyl-2,3-dihydro-1H-cyclopenta[b]quinoline}

(9g):

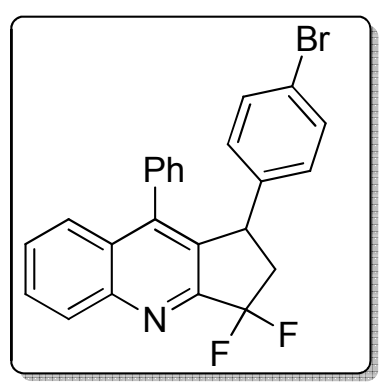

White solid (78 mg, 90\% yield); ${ }^{1} \mathrm{H}$ NMR (400 MHz, $\mathrm{CDCl}_{3}$ ) $\delta 8.34(\mathrm{~d}, J=8.5 \mathrm{~Hz}, 1 \mathrm{H}), 7.76(\mathrm{ddd}, J=8.4,6.7,1.4 \mathrm{~Hz}$, 1H), 7.57 (dd, $J=8.5,0.9 \mathrm{~Hz}, 1 \mathrm{H}), 7.51-7.41(\mathrm{~m}, 2 \mathrm{H}), 7.34$ (tt, $J=7.5,1.2 \mathrm{~Hz}, 1 \mathrm{H}), 7.27-7.22(\mathrm{~m}, 1 \mathrm{H}), 7.16-7.09(\mathrm{~m}$, $3 \mathrm{H}), 6.59-6.53(\mathrm{~m}, 3 \mathrm{H}), 4.52(\mathrm{dt}, J=9.0,4.4 \mathrm{~Hz}, 1 \mathrm{H}), 3.21$ (dddd, $J=16.9,15.3,12.3,9.2 \mathrm{~Hz}, 1 \mathrm{H}), 2.73-2.60(\mathrm{~m}, 1 \mathrm{H})$;

${ }^{13} \mathrm{C}$ NMR $\left(101 \mathrm{MHz}, \mathrm{CDCl}_{3}\right) \delta 155.12(\mathrm{t}, J=24.5 \mathrm{~Hz}), 149.44,147.33,142.16$, 134.66, 134.43 (t, $J=3.6 \mathrm{~Hz}), 131.37,130.48,130.06,129.41,129.30,128.92$, 128.63, 128.44, 128.30, 128.19, 127.87, 126.00, 125.84 (t, $J=247.4 \mathrm{~Hz}), 120.41$, $44.02(\mathrm{t}, J=23.5 \mathrm{~Hz}), 42.63 ;{ }^{19} \mathrm{~F} \mathrm{NMR}\left(377 \mathrm{MHz}, \mathrm{CDCl}_{3}\right) \delta-89.04(\mathrm{~d}, J=259.9 \mathrm{~Hz}$, $1 \mathrm{~F}),-93.80$ (d, $J=260.0 \mathrm{~Hz}, 1 \mathrm{~F})$; EI-MS (m/z, relative intensity): $437\left({ }^{81} \mathrm{Br}, \mathrm{M}^{+}, 65\right)$, $435\left({ }^{79} \mathrm{Br}, \mathrm{M}^{+}, 69\right), 356$ (100), 336 (30), 279 (21); HRMS (EI) calcd. for $\mathrm{C}_{24} \mathrm{H}_{16} \mathrm{~N}_{1} \mathrm{Br}_{1} \mathrm{~F}_{2}[\mathrm{M}]^{+}$435.0429, found: 435.0425 .

\section{4-(3,3-Difluoro-9-phenyl-2,3-dihydro-1H-cyclopenta[b]quinolin-1-yl)benzonitrile} (9h): 


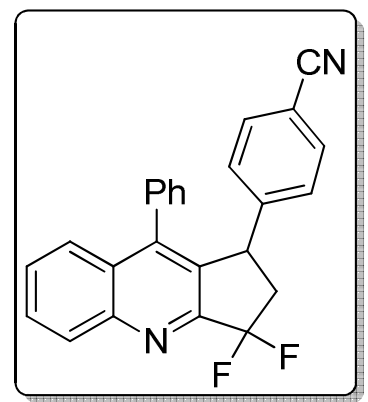

White solid (44 mg, 58\% yield); ${ }^{1} \mathrm{H}$ NMR (400 MHz, $\mathrm{CDCl}_{3}$ ) $\delta 8.35(\mathrm{~d}, J=8.5 \mathrm{~Hz}, 1 \mathrm{H}), 7.79(\mathrm{ddd}, J=8.4,6.7,1.5 \mathrm{~Hz}, 1 \mathrm{H})$, $7.58(\mathrm{dd}, J=8.5,1.0 \mathrm{~Hz}, 1 \mathrm{H}), 7.54-7.49(\mathrm{~m}, 1 \mathrm{H}), 7.45$ (td, $J$ $=7.6,0.8 \mathrm{~Hz}, 1 \mathrm{H}), 7.37-7.27(\mathrm{~m}, 4 \mathrm{H}), 7.10(\mathrm{td}, J=7.6,0.8$ $\mathrm{Hz}, 1 \mathrm{H}), 6.84-6.77(\mathrm{~m}, 2 \mathrm{H}), 6.53(\mathrm{~d}, J=7.7 \mathrm{~Hz}, 1 \mathrm{H}), 4.63$ (dt, $J=9.0,4.4 \mathrm{~Hz}, 1 \mathrm{H}), 3.25$ (dddd, $J=16.6,15.5,11.9,9.3$ $\mathrm{Hz}, 1 \mathrm{H}), 2.68$ (tdd, $J=15.6,10.9,4.6 \mathrm{~Hz}, 1 \mathrm{H}) ;{ }^{13} \mathrm{C} \mathrm{NMR}\left(101 \mathrm{MHz}, \mathrm{CDCl}_{3}\right) \delta$ $154.84(\mathrm{t}, J=24.7 \mathrm{~Hz}), 149.48,148.33(\mathrm{~d}, J=1.4 \mathrm{~Hz}), 147.27,134.47,133.65$ (t, $J=$ $4.1 \mathrm{~Hz}), 132.06,130.48,130.28,129.11,128.73$ (d, $J=2.6 \mathrm{~Hz}), 128.44,128.34$, 128.20, 128.04, $125.70(\mathrm{t}, J=265.1 \mathrm{~Hz}), 118.62,110.39,43.72(\mathrm{t}, J=24.0 \mathrm{~Hz})$, $43.09(\mathrm{t}, J=3.2 \mathrm{~Hz}) ;{ }^{19} \mathrm{~F}$ NMR $\left(377 \mathrm{MHz}, \mathrm{CDCl}_{3}\right) \delta-89.00(\mathrm{~d}, J=260.4 \mathrm{~Hz}, 1 \mathrm{~F})$, -93.58 (d, $J=260.5 \mathrm{~Hz}, 1 \mathrm{~F})$; EI-MS (m/z, relative intensity): $382\left(\mathrm{M}^{+}, 100\right), 362$ (31), 305 (33), 281 (14), 207 (83); HRMS (EI) calcd. for $\mathrm{C}_{25} \mathrm{H}_{16} \mathrm{~N}_{2} \mathrm{~F}_{2}[\mathrm{M}]^{+}$382.1276, found: 382.1273 .

\section{1-Butoxy-3,3-difluoro-9-phenyl-2,3-dihydro-1H-cyclopenta[b]quinoline (9i):}

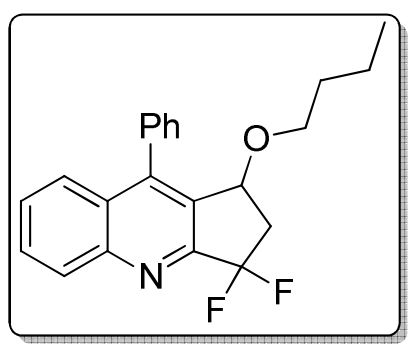

White solid (49 mg, 70\% yield); ${ }^{1} \mathrm{H}$ NMR (400 MHz, $\left.\mathrm{CDCl}_{3}\right) \delta 8.32(\mathrm{~d}, J=8.8 \mathrm{~Hz}, 1 \mathrm{H}), 7.82-7.73(\mathrm{~m}, 2 \mathrm{H})$, $7.62-7.48(\mathrm{~m}, 5 \mathrm{H}), 7.39-7.30(\mathrm{~m}, 1 \mathrm{H}), 4.94-4.87(\mathrm{~m}$, $1 \mathrm{H}), 3.37(\mathrm{dt}, J=8.8,6.4 \mathrm{~Hz}, 1 \mathrm{H}), 3.09(\mathrm{dt}, J=8.8,6.5$ $\mathrm{Hz}, 1 \mathrm{H}), 2.82$ (dddd, $J=17.7,10.8,10.1,4.5 \mathrm{~Hz}, 2 \mathrm{H}$ ), $1.36-1.27(\mathrm{~m}, 2 \mathrm{H}), 1.20-1.08(\mathrm{~m}, 2 \mathrm{H}), 0.80(\mathrm{t}, J=7.3 \mathrm{~Hz}, 3 \mathrm{H}) ;{ }^{13} \mathrm{C}$ NMR $(101$ $\left.\mathrm{MHz}, \mathrm{CDCl}_{3}\right) \delta 155.08(\mathrm{t}, J=24.7 \mathrm{~Hz}), 150.02,148.24,134.85,131.47(\mathrm{t}, J=3.9$ $\mathrm{Hz}), 130.50,130.45$ (d, $J=2.5 \mathrm{~Hz}), 128.74,128.62,128.46,127.85,126.59,125.00$ (dd, $J=249.9,246.6 \mathrm{~Hz}), 74.52$ (d, $J=5.6 \mathrm{~Hz}), 69.82,41.67$ (t, $J=22.4 \mathrm{~Hz}), 31.85$, 19.25, 13.95; ${ }^{19} \mathrm{~F}$ NMR (377 MHz, $\left.\mathrm{CDCl}_{3}\right) \delta-88.85(\mathrm{~d}, J=259.8 \mathrm{~Hz}, 1 \mathrm{~F}),-97.55(\mathrm{~d}, J$ $=259.8 \mathrm{~Hz}, 1 \mathrm{~F})$; EI-MS (m/z, relative intensity): $353\left(\mathrm{M}^{+}, 6\right), 296$ (9), 281 (91), 280 (100); HRMS (EI) calcd. for $\mathrm{C}_{22} \mathrm{H}_{21} \mathrm{O}_{1} \mathrm{~N}_{1} \mathrm{~F}_{2}[\mathrm{M}]^{+}$353.1586, found: 353.1588.

\section{1-(3,3-Difluoro-9-phenyl-2,3-dihydro-1 $H$-cyclopenta $[b]$ quinolin-1-yl)pyrrolidin-2}




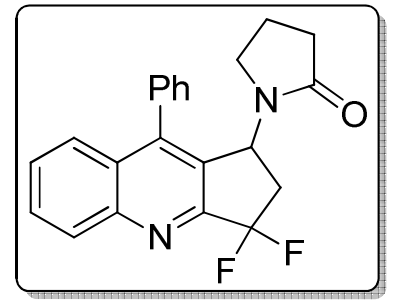

White solid (56 mg, 77\% yield); ${ }^{1} \mathrm{H}$ NMR (400 MHz, $\left.\mathrm{CDCl}_{3}\right) \delta 8.31(\mathrm{~d}, J=8.4 \mathrm{~Hz}, 1 \mathrm{H}), 7.82-7.75(\mathrm{~m}, 2 \mathrm{H})$, $7.60-7.52(\mathrm{~m}, 2 \mathrm{H}), 7.52-7.47(\mathrm{~m}, 2 \mathrm{H}), 7.40(\mathrm{~d}, J=7.5$ $\mathrm{Hz}, 1 \mathrm{H}), 7.31(\mathrm{~d}, J=3.3 \mathrm{~Hz}, 1 \mathrm{H}), 6.15-6.08(\mathrm{~m}, 1 \mathrm{H}), 3.13$ $-2.97(\mathrm{~m}, 2 \mathrm{H}), 2.60-2.44(\mathrm{~m}, 2 \mathrm{H}), 2.06(\mathrm{ddd}, J=13.4$, 9.3, 4.3 Hz, 1H), $1.76-1.53(\mathrm{~m}, 2 \mathrm{H}), 1.36(\mathrm{dtt}, J=11.6,8.8,5.8 \mathrm{~Hz}, 1 \mathrm{H}) .{ }^{13} \mathrm{C} \mathrm{NMR}$ $\left(101 \mathrm{MHz}, \mathrm{CDCl}_{3}\right) \delta 174.32,154.91(\mathrm{t}, J=24.3 \mathrm{~Hz}), 149.96,146.83,133.42,130.56$ $(\mathrm{d}, J=2.8 \mathrm{~Hz}), 129.68,129.23,129.10,128.29(\mathrm{~d}, J=8.2 \mathrm{~Hz}), 128.14,128.06(\mathrm{t}, J=$ $3.8 \mathrm{~Hz}), 127.93,126.09,123.95(\mathrm{t}, J=247.5 \mathrm{~Hz}), 48.01(\mathrm{t}, J=4.1 \mathrm{~Hz}), 42.32,38.97$ $(\mathrm{t}, J=24.1 \mathrm{~Hz}), 30.23,17.19 ;{ }^{19} \mathrm{~F}$ NMR $\left(377 \mathrm{MHz}, \mathrm{CDCl}_{3}\right) \delta-92.77(\mathrm{~d}, J=260.9 \mathrm{~Hz}$, 1F), -94.04 (d, $J=260.8 \mathrm{~Hz}, 1 \mathrm{~F})$; EI-MS (m/z, relative intensity): 364 (M+12), 344 (100), 316 (25), 259 (28); HRMS (EI) calcd. for $\mathrm{C}_{22} \mathrm{H}_{18} \mathrm{O}_{1} \mathrm{~N}_{1} \mathrm{~F}_{2}[\mathrm{M}]^{+}$364.1382, found: 364.1380 .

Methyl 3,3-difluoro-9-phenyl-2,3-dihydro-1 $H$-cyclopenta[b]quinoline-1-carboxyl -ate (9k):

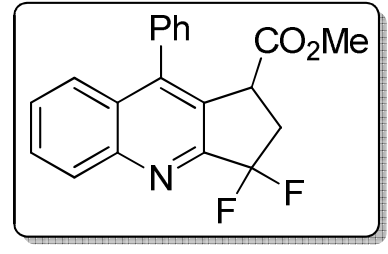

White solid (66 mg, 97\% yield); ${ }^{1} \mathrm{H}$ NMR (400 MHz, $\left.\mathrm{CDCl}_{3}\right) \delta 8.31(\mathrm{~d}, J=8.5 \mathrm{~Hz}, 1 \mathrm{H}), 7.77(\mathrm{ddd}, J=8.3,6.9$, $1.2 \mathrm{~Hz}, 1 \mathrm{H}), 7.67$ (d, $J=8.0 \mathrm{~Hz}, 1 \mathrm{H}), 7.56-7.49(\mathrm{~m}, 4 \mathrm{H})$, $7.30(\mathrm{ddd}, J=8.9,5.8,1.5 \mathrm{~Hz}, 2 \mathrm{H}), 4.26(\mathrm{ddd}, J=8.9,5.9$, $3.2 \mathrm{~Hz}, 1 \mathrm{H}), 3.31(\mathrm{~s}, 3 \mathrm{H}), 3.10-2.96(\mathrm{~m}, 1 \mathrm{H}), 2.85(\mathrm{dd}, J=14.6,5.9 \mathrm{~Hz}, 1 \mathrm{H}) ;{ }^{13} \mathrm{C}$ NMR (101 MHz, $\left.\mathrm{CDCl}_{3}\right) \delta 172.33,154.96$ (t, $\left.J=24.4 \mathrm{~Hz}\right), 149.48,147.06,134.53$, $130.60,130.30,129.81,129.43(\mathrm{t}, J=4.3 \mathrm{~Hz}), 129.16,128.94,128.75,128.53$ (d, $J=$ $4.4 \mathrm{~Hz}), 128.07,126.22,125.00(\mathrm{dd}, J=250.1,245.5 \mathrm{~Hz}), 52.39,42.63(\mathrm{t}, J=3.0$ $\mathrm{Hz}), 39.45(\mathrm{t}, J=25.4 \mathrm{~Hz}) ;{ }^{19} \mathrm{~F}$ NMR $\left(377 \mathrm{MHz}, \mathrm{CDCl}_{3}\right) \delta-92.52(\mathrm{~d}, J=258.6 \mathrm{~Hz}$, 1F), -95.06 (d, $J=258.6 \mathrm{~Hz}, 1 \mathrm{~F})$; EI-MS (m/z, relative intensity): 339 (M+ 36 ), 280 (100), 260 (16), 216 (12); HRMS (EI) calcd. for $\mathrm{C}_{20} \mathrm{H}_{15} \mathrm{O}_{2} \mathrm{~N}_{1} \mathrm{~F}_{2}[\mathrm{M}]^{+}$339.1065, found: 339.1064 . 


\section{1-Cyclohexyl-3,3-difluoro-9-phenyl-2,3-dihydro-1H-cyclopenta[b]quinoline (9l):}

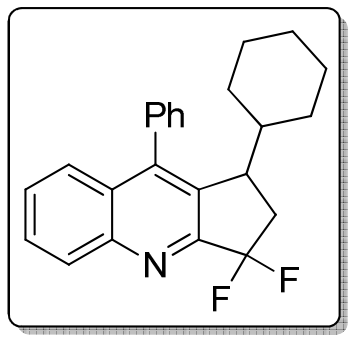

White solid (44 mg, 61\% yield); ${ }^{1} \mathrm{H} \mathrm{NMR}\left(400 \mathrm{MHz}, \mathrm{CDCl}_{3}\right)$ $\delta 8.30(\mathrm{~d}, J=8.4 \mathrm{~Hz}, 1 \mathrm{H}), 7.75-7.70(\mathrm{~m}, 1 \mathrm{H}), 7.69-7.65(\mathrm{~m}$, 1H), $7.59-7.52(\mathrm{~m}, 3 \mathrm{H}), 7.49$ (ddd, $J=8.3,6.8,1.1 \mathrm{~Hz}, 1 \mathrm{H})$, $7.38-7.32(\mathrm{~m}, 2 \mathrm{H}), 3.57(\mathrm{td}, \mathrm{J}=8.4,4.1 \mathrm{~Hz}, 1 \mathrm{H}), 2.73-2.46$ $(\mathrm{m}, 2 \mathrm{H}), 1.63-1.55(\mathrm{~m}, 1 \mathrm{H}), 1.54-1.44(\mathrm{~m}, 2 \mathrm{H}), 1.35(\mathrm{~d}, J=$ $11.9 \mathrm{~Hz}, 1 \mathrm{H}), 1.09(\mathrm{~m}, 1 \mathrm{H}), 1.03-0.91(\mathrm{~m}, 3 \mathrm{H}), 0.91-0.82(\mathrm{~m}, 1 \mathrm{H}), 0.80-0.66(\mathrm{~m}$, $2 \mathrm{H}) ;{ }^{13} \mathrm{C}$ NMR $\left(101 \mathrm{MHz}, \mathrm{CDCl}_{3}\right) \delta 155.96(\mathrm{t}, J=24.6 \mathrm{~Hz}), 149.06,146.21,135.80$, $133.61(\mathrm{t}, J=4.3 \mathrm{~Hz}), 130.51,129.56,129.34,128.69,128.57,128.39(\mathrm{~d}, J=11.6$ Hz), 127.65, 126.03 (dd, $J=257.6,255.7 \mathrm{~Hz}), 125.87,42.67$ (t, $J=2.9 \mathrm{~Hz}), 38.33$, $35.37(\mathrm{t}, J=23.2 \mathrm{~Hz}), 31.88,26.73,26.30,25.99,25.87 ;{ }^{19} \mathrm{~F} \mathrm{NMR}\left(377 \mathrm{MHz}, \mathrm{CDCl}_{3}\right)$ $\delta-90.70(\mathrm{~d}, J=258.9 \mathrm{~Hz}, 1 \mathrm{~F}),-91.53(\mathrm{~d}, J=258.9 \mathrm{~Hz}, 1 \mathrm{~F}) ;$ ESI-MS m/z: 364 $\left(\left[\mathrm{M}+\mathrm{H}^{+}\right]\right.$, 100); HRMS (ESI) calcd. for $\mathrm{C}_{24} \mathrm{H}_{24} \mathrm{~N}_{1} \mathrm{~F}_{2}[\mathrm{M}+\mathrm{H}]^{+}$364.1871, found: 364.1865 .

\section{(3,3-Difluoro-9-phenyl-2,3-dihydro-1H-cyclopenta[b]quinolin-1-yl)methanol}

(9m):

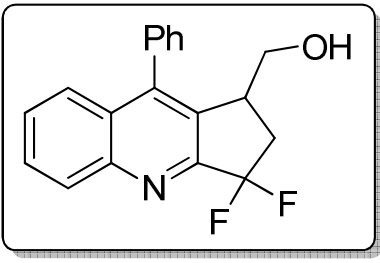

White solid (30 mg, 48\% yield); ${ }^{1} \mathrm{H}$ NMR (400 MHz, $\mathrm{CDCl}_{3}$ ) $\delta 8.30(\mathrm{~d}, J=8.5 \mathrm{~Hz}, 1 \mathrm{H}), 7.75(\mathrm{ddd}, J=8.4,6.8,1.3 \mathrm{~Hz}$, 1H), $7.64(\mathrm{~d}, J=7.7 \mathrm{~Hz}, 1 \mathrm{H}), 7.59-7.50(\mathrm{~m}, 4 \mathrm{H}), 7.41-$ $7.34(\mathrm{~m}, 2 \mathrm{H}), 3.66(\mathrm{dd}, J=11.8,7.0 \mathrm{~Hz}, 1 \mathrm{H}), 3.55-3.42(\mathrm{~m}$, 1H), 3.28 (dd, $J=14.4,8.8 \mathrm{~Hz}, 1 \mathrm{H}), 2.81$ (ddd, $J=18.4,9.1,3.5 \mathrm{~Hz}, 2 \mathrm{H}), 1.48$ (t, $J$ $=5.3 \mathrm{~Hz}, 1 \mathrm{H}) ;{ }^{13} \mathrm{C}$ NMR $\left(101 \mathrm{MHz}, \mathrm{CDCl}_{3}\right) \delta 156.05(\mathrm{t}, J=24.7 \mathrm{~Hz}), 149.16$, 146.67, 135.25, $131.20(\mathrm{t}, J=4.4 \mathrm{~Hz}), 130.51,129.97,129.68,129.43,128.98$, $128.79,128.50,128.35,127.92,125.74(\mathrm{t}, J=246.9 \mathrm{~Hz}), 63.64,39.92(\mathrm{~d}, J=4.4 \mathrm{~Hz})$, $37.63(\mathrm{t}, J=23.4 \mathrm{~Hz}) ;{ }^{19} \mathrm{~F}$ NMR $\left(377 \mathrm{MHz}, \mathrm{CDCl}_{3}\right) \delta-88.19(\mathrm{~d}, J=259.8 \mathrm{~Hz}, 1 \mathrm{~F})$, -95.49 (d, $J=259.9 \mathrm{~Hz}, 1 \mathrm{~F})$; EI-MS (m/z, relative intensity): $311\left(\mathrm{M}^{+}, 7\right), 281$ (37), 207 (100), 191 (12); HRMS (EI) calcd. for $\mathrm{C}_{19} \mathrm{H}_{15} \mathrm{O}_{1} \mathrm{~N}_{1} \mathrm{~F}_{2}[\mathrm{M}]^{+}$311.1116, found: 311.1115 . 


\section{3,3-Difluoro-1,1,9-triphenyl-2,3-dihydro-1H-cyclopenta[b]quinoline (9n):}

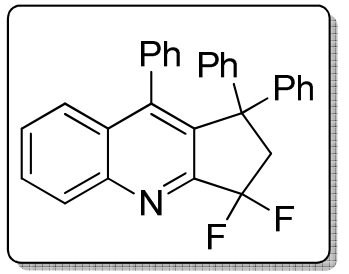

White solid (61 mg, 49\% yield); ${ }^{1} \mathrm{H}$ NMR (400 MHz, $\left.\mathrm{CDCl}_{3}\right) \delta$ $8.34(\mathrm{~d}, J=8.5 \mathrm{~Hz}, 1 \mathrm{H}), 7.75(\mathrm{t}, J=7.7 \mathrm{~Hz}, 1 \mathrm{H}), 7.41(\mathrm{t}, J=$ $7.7 \mathrm{~Hz}, 1 \mathrm{H}), 7.24(\mathrm{~s}, 1 \mathrm{H}), 7.12-7.03(\mathrm{~m}, 7 \mathrm{H}), 6.89(\mathrm{t}, J=7.0$ $\mathrm{Hz}, 6 \mathrm{H}), 6.53(\mathrm{~d}, J=7.7 \mathrm{~Hz}, 2 \mathrm{H}), 3.48$ (t, $J=13.1 \mathrm{~Hz}, 2 \mathrm{H})$;

${ }^{13} \mathrm{C}$ NMR $\left(101 \mathrm{MHz}, \mathrm{CDCl}_{3}\right) \delta 155.29$ (t, $\left.J=24.2 \mathrm{~Hz}\right), 149.11,148.37,144.71$, $137.03(\mathrm{t}, J=3.9 \mathrm{~Hz}), 134.36,130.34,130.23,130.16,129.27,128.79,127.90$, 127.77, 127.59, 127.07, 126.63, 124.37 (t, $J=247.5 \mathrm{~Hz}), 57.04$ (t, $J=3.1 \mathrm{~Hz}), 55.97$ $(\mathrm{t}, J=21.4 \mathrm{~Hz}) ;{ }^{19} \mathrm{~F}$ NMR $\left(377 \mathrm{MHz}, \mathrm{CDCl}_{3}\right) \delta-94.08(\mathrm{~s}, 2 \mathrm{~F})$; EI-MS (m/z, relative intensity): $433\left(\mathrm{M}^{+}, 100\right), 413$ (37), 356 (71), 336 (36); HRMS (EI) calcd. for $\mathrm{C}_{30} \mathrm{H}_{21} \mathrm{~N}_{1} \mathrm{~F}_{2}[\mathrm{M}]^{+}$433.1637, found: 433.1634 .

\section{3,3-Difluoro-1-methyl-9-phenyl-1-(prop-1-en-2-yl)-2,3-dihydro-1 $H$-cyclopenta[b]} quinoline (9o):

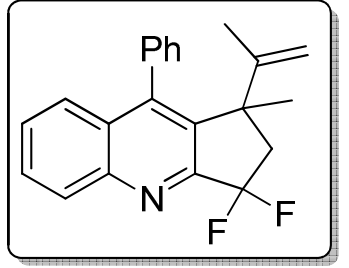

White solid (35 mg, 52\% yield); ${ }^{1} \mathrm{H} \mathrm{NMR}\left(400 \mathrm{MHz}, \mathrm{CDCl}_{3}\right) \delta$ $8.31(\mathrm{~d}, J=8.5 \mathrm{~Hz}, 1 \mathrm{H}), 7.73(\mathrm{ddd}, J=8.3,6.6,1.6 \mathrm{~Hz}, 1 \mathrm{H})$, $7.49-7.38(\mathrm{~m}, 5 \mathrm{H}), 7.23(\mathrm{dd}, J=6.9,1.1 \mathrm{~Hz}, 1 \mathrm{H}), 7.21-7.15$ $(\mathrm{m}, 1 \mathrm{H}), 4.64(\mathrm{~s}, 1 \mathrm{H}), 4.42(\mathrm{~s}, 1 \mathrm{H}), 2.91-2.75(\mathrm{~m}, 1 \mathrm{H}), 2.44$ (ddd, $J=17.4,15.1,10.3 \mathrm{~Hz}, 1 \mathrm{H}), 1.59$ (s, 3H), 1.34 (s, 3H); ${ }^{13} \mathrm{C}$ NMR (101 MHz, $\left.\mathrm{CDCl}_{3}\right) \delta 155.41(\mathrm{t}, J=24.6 \mathrm{~Hz}), 148.91,147.85(\mathrm{~d}, J=2.5 \mathrm{~Hz}), 146.59,137.22(\mathrm{t}, J$ $=4.3 \mathrm{~Hz}$ ), 134.82, 130.31, 129.91, 129.76, $129.22,128.69$ (d, $J=12.2 \mathrm{~Hz}), 128.42$, 128.02, 127.78, 127.66, 126.39, 124.80 (dd, $J=247.8,245.5 \mathrm{~Hz}), 113.09,48.47$ (t, $J$ $=21.6 \mathrm{~Hz}), 48.00(\mathrm{t}, J=3.0 \mathrm{~Hz}), 26.86(\mathrm{~d}, J=2.5 \mathrm{~Hz}), 20.17 ;{ }^{19} \mathrm{~F}$ NMR $(377 \mathrm{MHz}$, $\left.\mathrm{CDCl}_{3}\right) \delta-89.57(\mathrm{~d}, J=259.8 \mathrm{~Hz}, 1 \mathrm{~F}),-92.64(\mathrm{~d}, J=259.7 \mathrm{~Hz}, 1 \mathrm{~F}) ;$ EI-MS $(\mathrm{m} / \mathrm{z}$, relative intensity): $335\left(\mathrm{M}^{+}, 49\right), 334$ (93), 294 (71), 254 (44), 207 (74); HRMS (EI) calcd. for $\mathrm{C}_{22} \mathrm{H}_{19} \mathrm{~N}_{1} \mathrm{~F}_{2}[\mathrm{M}]^{+}$335.1480, found: 335.1482 .

\section{trans-3,3-Difluoro-1,2,9-triphenyl-2,3-dihydro-1H-cyclopenta[b]quinoline (9p):}

White solid (46 mg, 54\% yield); ${ }^{1} \mathrm{H} \mathrm{NMR}\left(400 \mathrm{MHz}, \mathrm{CDCl}_{3}\right) \delta 8.38(\mathrm{~d}, J=8.5 \mathrm{~Hz}$, 1H), $7.79(\mathrm{ddd}, J=8.4,6.8,1.3 \mathrm{~Hz}, 1 \mathrm{H}), 7.62(\mathrm{~d}, J=8.4 \mathrm{~Hz}, 1 \mathrm{H}), 7.54-7.47$ (m, 


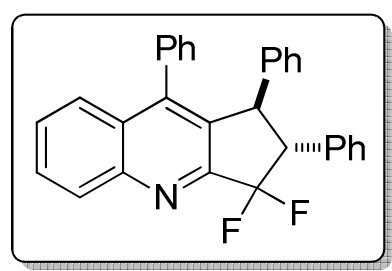

1H), $7.36-7.29(\mathrm{~m}, 6 \mathrm{H}), 7.20(\mathrm{ddd}, J=8.6,6.5,2.7 \mathrm{~Hz}$, 2H), $6.99(\mathrm{dd}, J=12.8,5.1 \mathrm{~Hz}, 1 \mathrm{H}), 6.96-6.88(\mathrm{~m}, 3 \mathrm{H})$, $6.72-6.64(\mathrm{~m}, 3 \mathrm{H}), 4.75(\mathrm{dd}, J=8.0,2.2 \mathrm{~Hz}, 1 \mathrm{H}), 3.97$ (ddd, $J=17.4,14.8,8.1 \mathrm{~Hz}, 1 \mathrm{H}) ;{ }^{13} \mathrm{C} \mathrm{NMR}(101 \mathrm{MHz}$,

$\left.\mathrm{CDCl}_{3}\right) \delta 155.34(\mathrm{t}, J=24.1 \mathrm{~Hz}), 149.42,147.27,141.05,134.73,134.12,133.94(\mathrm{t}, J$ $=3.9 \mathrm{~Hz}), 130.58,129.97,129.48,129.23,128.71,128.64,128.43,128.26,128.13$, 128.06, 127.96, $127.87(\mathrm{~d}, J=4.1 \mathrm{~Hz}), 126.65,126.17,123.88$ (dd, $J=252.2,249.6$ $\mathrm{Hz}), 61.91(\mathrm{t}, J=21.4 \mathrm{~Hz}), 51.22(\mathrm{~d}, J=4.7 \mathrm{~Hz}) ;{ }^{19} \mathrm{~F}$ NMR $\left(377 \mathrm{MHz}, \mathrm{CDCl}_{3}\right) \delta$ $-98.75(\mathrm{~d}, J=256.3 \mathrm{~Hz}, 1 \mathrm{~F}),-100.45(\mathrm{~d}, J=256.5 \mathrm{~Hz}, 1 \mathrm{~F})$; EI-MS (m/z, relative intensity): $433\left(\mathrm{M}^{+}, 18\right), 355$ (14), 281 (22), 207 (100); HRMS (EI) calcd. for $\mathrm{C}_{30} \mathrm{H}_{21} \mathrm{~N}_{1} \mathrm{~F}_{2}[\mathrm{M}]^{+}$433.1637, found: 433.1635 .

6,6-Difluoro-12-phenyl-6,6a,7,11b-tetrahydrobenzo[5,6]pentaleno[2,1-b]quinolin e (9q):

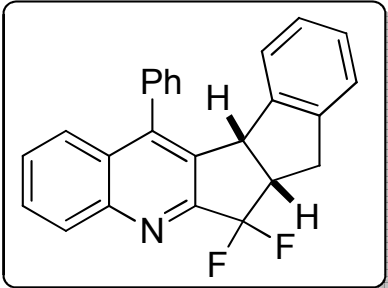

White solid (35 mg, 47\% yield); ${ }^{1} \mathrm{H}$ NMR (400 MHz, $\left.\mathrm{CDCl}_{3}\right) \delta 8.29(\mathrm{~d}, J=8.4 \mathrm{~Hz}, 1 \mathrm{H}), 7.80-7.71(\mathrm{~m}, 2 \mathrm{H})$, $7.67-7.55(\mathrm{~m}, 4 \mathrm{H}), 7.54-7.49(\mathrm{~m}, 1 \mathrm{H}), 7.43-7.38(\mathrm{~m}$, 1H), $7.13(\mathrm{~d}, J=7.5 \mathrm{~Hz}, 1 \mathrm{H}), 7.04$ (t, $J=7.4 \mathrm{~Hz}, 1 \mathrm{H}), 6.77$ $(\mathrm{t}, J=7.4 \mathrm{~Hz}, 1 \mathrm{H}), 5.97(\mathrm{~d}, J=7.7 \mathrm{~Hz}, 1 \mathrm{H}), 5.22(\mathrm{dd}, J=$ 7.7, $2.9 \mathrm{~Hz}, 1 \mathrm{H}), 3.72$ (dddd, $J=16.8,15.0,7.6,5.0 \mathrm{~Hz}, 1 \mathrm{H}), 3.37$ (dd, $J=16.8,4.8$ $\mathrm{Hz}, 1 \mathrm{H}), 3.23(\mathrm{dd}, J=16.9,9.2 \mathrm{~Hz}, 1 \mathrm{H}) ;{ }^{13} \mathrm{C} \mathrm{NMR}\left(101 \mathrm{MHz}, \mathrm{CDCl}_{3}\right) \delta 155.44(\mathrm{~d}, J$ $=25.1 \mathrm{~Hz}), 149.11,146.45,142.38,141.47,135.74,132.41(\mathrm{t}, J=4.4 \mathrm{~Hz}), 131.26$, $130.54,129.85,129.79,129.10,128.67,127.85,127.65,126.52,126.07,125.52$ (dd, $J$ $=252.6,245.3 \mathrm{~Hz}), 125.32,124.63,49.87(\mathrm{dd}, J=24.6,19.9 \mathrm{~Hz}), 49.43(\mathrm{~d}, J=2.6$ $\mathrm{Hz}), 31.21(\mathrm{dd}, J=7.7,2.7 \mathrm{~Hz}) ;{ }^{19} \mathrm{~F}$ NMR $\left(377 \mathrm{MHz}, \mathrm{CDCl}_{3}\right) \delta-91.50(\mathrm{~d}, J=260.3$ $\mathrm{Hz}, 1 \mathrm{~F}),-104.03$ (d, $J=260.3 \mathrm{~Hz}, 1 \mathrm{~F})$; EI-MS (m/z, relative intensity): $369\left(\mathrm{M}^{+}, 3\right)$, 341 (7), 281 (24), 207 (100); HRMS (EI) calcd. for $\mathrm{C}_{25} \mathrm{H}_{17} \mathrm{~N}_{1} \mathrm{~F}_{2}[\mathrm{M}]^{+}$369.1324, found: 369.1322 .

5,5-Difluoro-11-phenyl-2,3,4,4a,5,11b-hexahydropyrano[ $\left[2^{\prime}, 3^{\prime}: 3,4\right]$ cyclopenta[1,2- 


\section{b]quinoline (9r):}

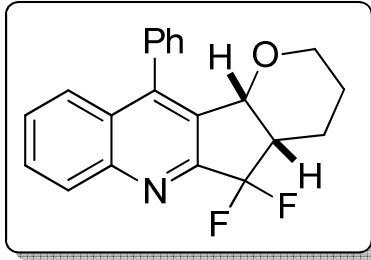

White solid (42 mg, 62\% yield); ${ }^{1} \mathrm{H}$ NMR (400 MHz, $\mathrm{CDCl}_{3}$ ) $\delta 8.32(\mathrm{~d}, J=8.4 \mathrm{~Hz}, 1 \mathrm{H}), 7.83(\mathrm{dd}, J=8.5,0.8 \mathrm{~Hz}, 1 \mathrm{H})$, $7.80-7.73(\mathrm{~m}, 2 \mathrm{H}), 7.60-7.51(\mathrm{~m}, 4 \mathrm{H}), 7.38(\mathrm{dd}, J=4.4$, $1.8 \mathrm{~Hz}, 1 \mathrm{H}), 4.71(\mathrm{td}, J=4.6,2.9 \mathrm{~Hz}, 1 \mathrm{H}), 3.96(\mathrm{dd}, J=9.7$, $3.6 \mathrm{~Hz}, 1 \mathrm{H}), 3.45$ (ddd, $J=11.3,6.7,2.0 \mathrm{~Hz}, 1 \mathrm{H}), 2.69-2.55(\mathrm{~m}, 1 \mathrm{H}), 2.39$ (d, $J=$ $10.8 \mathrm{~Hz}, 1 \mathrm{H}), 2.00-1.89(\mathrm{~m}, 2 \mathrm{H}), 1.58-1.48(\mathrm{~m}, 1 \mathrm{H}) ;{ }^{13} \mathrm{C} \mathrm{NMR}\left(101 \mathrm{MHz}, \mathrm{CDCl}_{3}\right)$ $\delta 156.53(\mathrm{t}, J=24.6 \mathrm{~Hz}), 149.74,147.53,134.29,131.05(\mathrm{t}, J=4.4 \mathrm{~Hz}), 130.73$, $130.59,130.29,129.86,128.93,128.76,128.14$ (d, $J=8.7 \mathrm{~Hz}), 127.82,126.80$, $125.18(\mathrm{dd}, J=252.8,250.0 \mathrm{~Hz}), 73.25(\mathrm{~d}, J=9.5 \mathrm{~Hz}), 67.07,43.39$ (t, $J=21.2 \mathrm{~Hz})$, $21.98(\mathrm{~d}, J=2.7 \mathrm{~Hz}), 19.44 ;{ }^{19} \mathrm{~F} \mathrm{NMR}\left(377 \mathrm{MHz}, \mathrm{CDCl}_{3}\right) \delta-101.08(\mathrm{~d}, J=257.9 \mathrm{~Hz}$, 1F), -107.11 (d, $J=257.8 \mathrm{~Hz}, 1 \mathrm{~F})$; EI-MS (m/z, relative intensity): 337 (85), 308 (92), 279 (31), 260 (100), 258 (33); HRMS (EI) calcd. for $\mathrm{C}_{21} \mathrm{H}_{17} \mathrm{O}_{1} \mathrm{~N}_{1} \mathrm{~F}_{2}[\mathrm{M}]^{+}$337.1273, found: 337.1270 .

\section{6,6-Difluoro-11-phenyl-6a,7,8,9,10,10a-hexahydro-6H-7,10-methanoindeno[2,1-b]} quinoline (9s):

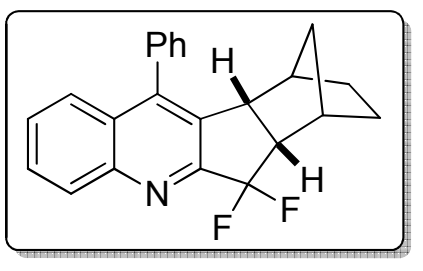

White solid (43 mg, 61\% yield); ${ }^{1} \mathrm{H}$ NMR (400 MHz, $\left.\mathrm{CDCl}_{3}\right) \delta 8.30(\mathrm{~d}, J=8.5 \mathrm{~Hz}, 1 \mathrm{H}), 7.76-7.66(\mathrm{~m}, 2 \mathrm{H})$, $7.60-7.45(\mathrm{~m}, 4 \mathrm{H}), 7.39(\mathrm{dd}, J=10.4,4.0 \mathrm{~Hz}, 2 \mathrm{H}), 3.40$ (dd, $J=7.3,4.9 \mathrm{~Hz}, 1 \mathrm{H}), 2.75(\mathrm{~d}, J=3.2 \mathrm{~Hz}, 1 \mathrm{H}), 2.67$

(ddd, $J=24.0,7.5,2.6 \mathrm{~Hz}, 1 \mathrm{H}), 1.85$ (d, $J=3.7 \mathrm{~Hz}, 1 \mathrm{H}), 1.56$ (tt, $J=12.0,4.6 \mathrm{~Hz}$, 1H), 1.37 (tt, $J=12.1,4.3 \mathrm{~Hz}, 1 \mathrm{H}), 1.29-1.20(\mathrm{~m}, 1 \mathrm{H}), 1.13-1.03(\mathrm{~m}, 1 \mathrm{H}), 0.92(\mathrm{~m}$, $2 \mathrm{H}) ;{ }^{13} \mathrm{C} \mathrm{NMR}\left(101 \mathrm{MHz}, \mathrm{CDCl}_{3}\right) \delta 156.81(\mathrm{t}, J=25.5 \mathrm{~Hz}), 149.40,146.19,135.60$, $133.80(\mathrm{t}, J=4.6 \mathrm{~Hz}), 130.59$, 130.06, 129.60, 129.29, 128.71, 128.52, 127.63, 125.92, 124.36 (dd, $J=373.3,129.5 \mathrm{~Hz}), 52.52$ (dd, $J=24.4,20.6 \mathrm{~Hz}), 48.32,40.03$, $37.08(\mathrm{dd}, J=7.0,3.5 \mathrm{~Hz}), 33.83,28.56,28.05 ;{ }^{19} \mathrm{~F} \mathrm{NMR}\left(377 \mathrm{MHz}, \mathrm{CDCl}_{3}\right) \delta$ $-77.55(\mathrm{~d}, J=266.7 \mathrm{~Hz}, 1 \mathrm{~F}),-104.37$ (d, $J=266.7 \mathrm{~Hz}, 1 \mathrm{~F})$; EI-MS (m/z, relative intensity): $347\left(\mathrm{M}^{+}, 69\right), 279$ (100), 258 (16), 207 (51); HRMS (EI) calcd. for $\mathrm{C}_{23} \mathrm{H}_{19} \mathrm{~N}_{1} \mathrm{~F}_{2}[\mathrm{M}]^{+}$347.1480, found: 347.1478. 


\section{Ethyl 3-fluoro-1,9-diphenyl-1H-cyclopenta[b]quinoline-2-carboxylate (11):}

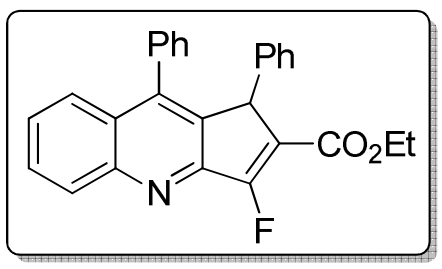

White solid (45 mg, 55\% yield); ${ }^{1} \mathrm{H}$ NMR (400 MHz, $\left.\mathrm{CDCl}_{3}\right) \delta 8.30(\mathrm{dd}, J=8.5,0.5 \mathrm{~Hz}, 1 \mathrm{H}), 7.74(\mathrm{ddd}, J=$ 8.4, 6.8, $1.5 \mathrm{~Hz}, 1 \mathrm{H}), 7.56(\mathrm{dd}, J=8.4,1.0 \mathrm{~Hz}, 1 \mathrm{H}), 7.52$ - $7.48(\mathrm{~m}, 1 \mathrm{H}), 7.47-7.43(\mathrm{~m}, 1 \mathrm{H}), 7.38-7.34(\mathrm{~m}, 2 \mathrm{H})$, $7.09-6.98(\mathrm{~m}, 2 \mathrm{H}), 6.96-6.89(\mathrm{~m}, 2 \mathrm{H}), 6.64-6.56(\mathrm{~m}, 2 \mathrm{H}), 6.43(\mathrm{~d}, J=7.8 \mathrm{~Hz}$, $1 \mathrm{H}), 4.91(\mathrm{~d}, J=6.8 \mathrm{~Hz}, 1 \mathrm{H}), 4.25-4.07(\mathrm{~m}, 2 \mathrm{H}), 1.18(\mathrm{t}, J=7.1 \mathrm{~Hz}, 3 \mathrm{H}) ;{ }^{13} \mathrm{C} \mathrm{NMR}$ $\left(101 \mathrm{MHz}, \mathrm{CDCl}_{3}\right) \delta 164.65,161.94(\mathrm{~d}, J=53.9 \mathrm{~Hz}), 153.49(\mathrm{~d}, J=20.4 \mathrm{~Hz}), 148.90$, $144.72(\mathrm{~d}, J=2.0 \mathrm{~Hz}), 136.05(\mathrm{~d}, J=2.2 \mathrm{~Hz}), 135.41(\mathrm{~d}, J=5.1 \mathrm{~Hz}), 130.00,129.83$, $129.19,128.98,128.58,128.23,128.18,128.09$, 127.93, 127.31, 126.85, 126.17, 123.10, 61.04, 47.93, 14.15; ${ }^{19} \mathrm{~F}$ NMR (377 MHz, $\left.\mathrm{CDCl}_{3}\right) \delta-117.66$ (s, 1F); EI-MS (m/z, relative intensity): 409 (47), 336 (100), 316 (18), 207 (61); HRMS (EI) calcd. for $\mathrm{C}_{27} \mathrm{H}_{20} \mathrm{O}_{2} \mathrm{~N}_{1} \mathrm{~F}_{1}[\mathrm{M}]^{+}$409.1473, found: 409.1473 .

6,6-Difluoro-11-phenyl-7,8,9,10-tetrahydro-6H-cyclohepta[b]quinoline-8,10-dicar bonitrile (13):

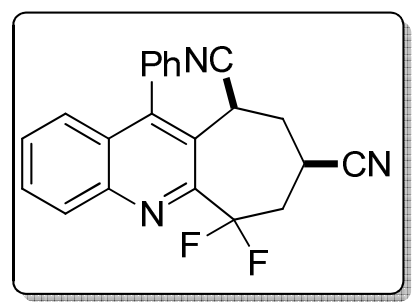

White solid (48 mg, 67\% yield); ${ }^{1} \mathrm{H}$ NMR (400 MHz, $\left.\mathrm{CDCl}_{3}\right) \delta 8.32(\mathrm{~d}, J=8.5 \mathrm{~Hz}, 1 \mathrm{H}), 7.76(\mathrm{ddd}, J=8.4,6.7$, $1.5 \mathrm{~Hz}, 1 \mathrm{H}), 7.55(\mathrm{dd}, J=8.5,1.0 \mathrm{~Hz}, 1 \mathrm{H}), 7.51-7.47(\mathrm{~m}$, $1 \mathrm{H}), 7.42(\mathrm{td}, J=7.6,0.8 \mathrm{~Hz}, 1 \mathrm{H}), 7.33-7.28(\mathrm{~m}, 1 \mathrm{H})$, $7.28-7.25(\mathrm{~m}, 2 \mathrm{H}), 7.23(\mathrm{~s}, 1 \mathrm{H}), 7.07(\mathrm{td}, J=7.6,0.8 \mathrm{~Hz}, 1 \mathrm{H}), 6.81-6.74(\mathrm{~m}, 2 \mathrm{H})$, $6.50(\mathrm{~d}, J=7.7 \mathrm{~Hz}, 1 \mathrm{H}), 4.60(\mathrm{dt}, J=9.0,4.4 \mathrm{~Hz}, 1 \mathrm{H}), 3.23$ (dddd, $J=16.6,15.5$, 11.9, $9.3 \mathrm{~Hz}, 1 \mathrm{H}), 2.66$ (tdd, $J=15.6,10.9,4.6 \mathrm{~Hz}, 1 \mathrm{H}) ;{ }^{13} \mathrm{C} \mathrm{NMR}(101 \mathrm{MHz}$, $\left.\mathrm{CDCl}_{3}\right) \delta 154.96(\mathrm{t}, J=24.7 \mathrm{~Hz}), 149.60,148.45(\mathrm{~d}, J=1.4 \mathrm{~Hz}), 147.40,134.60$, $133.77(\mathrm{t}, J=4.1 \mathrm{~Hz}), 132.18,130.60,130.40,129.23,128.87,128.84,128.56$, 128.46, 128.32, 128.16, 126.02, 124.47 (d, $J=247.4 \mathrm{~Hz}), 118.74,110.51,43.84(\mathrm{t}, J$ $=24.0 \mathrm{~Hz}), 43.21(\mathrm{t}, J=3.2 \mathrm{~Hz}) ;{ }^{19} \mathrm{~F} \mathrm{NMR}\left(377 \mathrm{MHz}, \mathrm{CDCl}_{3}\right)-89.00(\mathrm{~d}, J=260.4$ $\mathrm{Hz}, 1 \mathrm{~F}),-93.58$ (d, $J=260.5 \mathrm{~Hz}, 1 \mathrm{~F})$; EI-MS (m/z, relative intensity): $359\left(\mathrm{M}^{+}, 41\right)$, 305 (100), 258 (14); HRMS (EI) calcd. for $\mathrm{C}_{22} \mathrm{H}_{15} \mathrm{~N}_{3} \mathrm{~F}_{2}[\mathrm{M}]^{+}$359.1229, found: 359.1226. 
cis-3-Fluoro-1,9-diphenyl-2,3-dihydro-1 $H$-cyclopenta[b]quinoline (15):

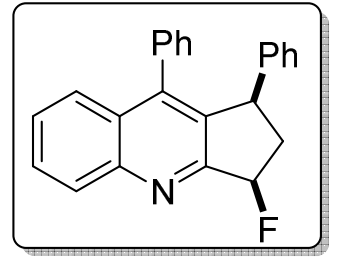

White solid (19 mg, 28\% yield); ${ }^{1} \mathrm{H}$ NMR (400 $\left.\mathrm{MHz}, \mathrm{CDCl}_{3}\right) \delta$ $8.27(\mathrm{~d}, J=8.5 \mathrm{~Hz}, 1 \mathrm{H}), 7.72(\mathrm{ddd}, J=8.4,6.8,1.4 \mathrm{~Hz}, 1 \mathrm{H})$, $7.59-7.52(\mathrm{~m}, 1 \mathrm{H}), 7.47-7.35(\mathrm{~m}, 2 \mathrm{H}), 7.25(\mathrm{ddt}, J=8.6,6.0$, $1.2 \mathrm{~Hz}, 2 \mathrm{H}), 7.06-6.95(\mathrm{~m}, 4 \mathrm{H}), 6.66(\mathrm{dt}, J=4.3,3.0 \mathrm{~Hz}, 2 \mathrm{H})$, $6.58(\mathrm{~d}, J=7.7 \mathrm{~Hz}, 1 \mathrm{H}), 6.33(\mathrm{ddd}, J=55.7,6.6,3.7 \mathrm{~Hz}, 1 \mathrm{H}), 4.68(\mathrm{dd}, J=7.4,6.0$ $\mathrm{Hz}, 1 \mathrm{H}), 2.91$ (dddd, $J=25.7,14.7,8.4,3.7 \mathrm{~Hz}, 1 \mathrm{H}), 2.65-2.49(\mathrm{~m}, 1 \mathrm{H}) ;{ }^{13} \mathrm{C} \mathrm{NMR}$ $\left(101 \mathrm{MHz}, \mathrm{CDCl}_{3}\right) \delta 160.94(\mathrm{~d}, J=15.1 \mathrm{~Hz}), 149.23,146.57,144.55,136.28(\mathrm{~d}, J=$ $2.7 \mathrm{~Hz}), 135.41,130.03,129.56,129.43,128.50,128.42,128.15,128.09,128.08$, 128.02, 127.92, 127.06, 126.15, 126.02, 94.86 (d, $J=178.2 \mathrm{~Hz}), 46.49,40.25$ (d, $J=$ $20.8 \mathrm{~Hz}) ;{ }^{19} \mathrm{~F}$ NMR (377 MHz, $\left.\mathrm{CDCl}_{3}\right) \delta$-171.17 (s); EI-MS (m/z, relative intensity): $339\left(\mathrm{M}^{+}, 100\right), 318$ (21), 262 (45), 242 (12); HRMS (EI) calcd. for $\mathrm{C}_{24} \mathrm{H}_{18} \mathrm{~N}_{1} \mathrm{~F}_{1}[\mathrm{M}]^{+}$ 339.1418, found: 339.1420 .

trans-3-Fluoro-1,9-diphenyl-2,3-dihydro-1H-cyclopenta[b]quinoline (15'):

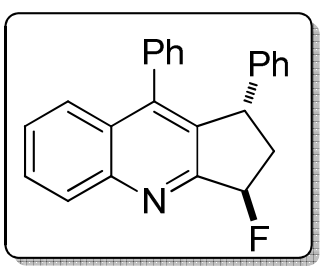

White solid (19 mg, 28\% yield); ${ }^{1} \mathrm{H} \mathrm{NMR}\left(400 \mathrm{MHz}, \mathrm{CDCl}_{3}\right) \delta$ $8.27(\mathrm{~d}, J=8.5 \mathrm{~Hz}, 1 \mathrm{H}), 7.72$ (ddd, $J=8.4,6.8,1.4 \mathrm{~Hz}, 1 \mathrm{H})$, $7.60-7.53(\mathrm{~m}, 1 \mathrm{H}), 7.47-7.34(\mathrm{~m}, 2 \mathrm{H}), 7.25(\mathrm{ddt}, J=8.6,6.0$, $1.2 \mathrm{~Hz}, 2 \mathrm{H}), 7.06-6.96(\mathrm{~m}, 4 \mathrm{H}), 6.70-6.62(\mathrm{~m}, 2 \mathrm{H}), 6.58(\mathrm{~d}, J$ $=7.7 \mathrm{~Hz}, 1 \mathrm{H}), 6.33(\mathrm{ddd}, J=55.7,6.6,3.7 \mathrm{~Hz}, 1 \mathrm{H}), 4.68(\mathrm{dd}, J=7.4,6.0 \mathrm{~Hz}, 1 \mathrm{H})$, 2.91 (dddd, $J=25.7,14.7,8.4,3.7 \mathrm{~Hz}, 1 \mathrm{H}), 2.65-2.46(\mathrm{~m}, 1 \mathrm{H}) ;{ }^{13} \mathrm{C}$ NMR $(101 \mathrm{MHz}$, $\left.\mathrm{CDCl}_{3}\right) \delta 161.34(\mathrm{~d}, J=15.0 \mathrm{~Hz}), 149.08,146.31,144.03,136.07(\mathrm{~d}, J=2.5 \mathrm{~Hz})$, $135.35,130.03,129.40,128.80,128.43,128.33,127.85,127.65,127.07,126.27$, 126.03, $95.04(\mathrm{~d}, J=176.7 \mathrm{~Hz}), 46.36,42.19(\mathrm{~d}, J=21.2 \mathrm{~Hz}) ;{ }^{19} \mathrm{~F}$ NMR $(377 \mathrm{MHz}$, $\left.\mathrm{CDCl}_{3}\right) \delta-171.17$ (s, 1F); EI-MS (m/z, relative intensity): $339\left(\mathrm{M}^{+}, 100\right), 318$ (57), 262 (94), 241 (38); HRMS (EI) calcd. for $\mathrm{C}_{24} \mathrm{H}_{18} \mathrm{~N}_{1} \mathrm{~F}_{1}[\mathrm{M}]^{+}$339.1418, found: 339.1419 . 


\section{References}

[1] Wu, Y.-M.; Zhang, M.; Li, Y.-Q. J. Fluorine Chem. 2006, 127, 218

[2] Broglia, M. F.; Bertolotti, S. G.; Previtali, C. M. Photochem. Photobiol. 2007, 83, 535

[3] Wayner, D. D. M.;Dannenberg, J. J.; Griller, D. Chem. Phys. Lett.1986, 131, 189. 


\section{${ }^{1}$ H NMR, ${ }^{13} \mathrm{C}$ NMR and ${ }^{19}$ F NMR spectrums}
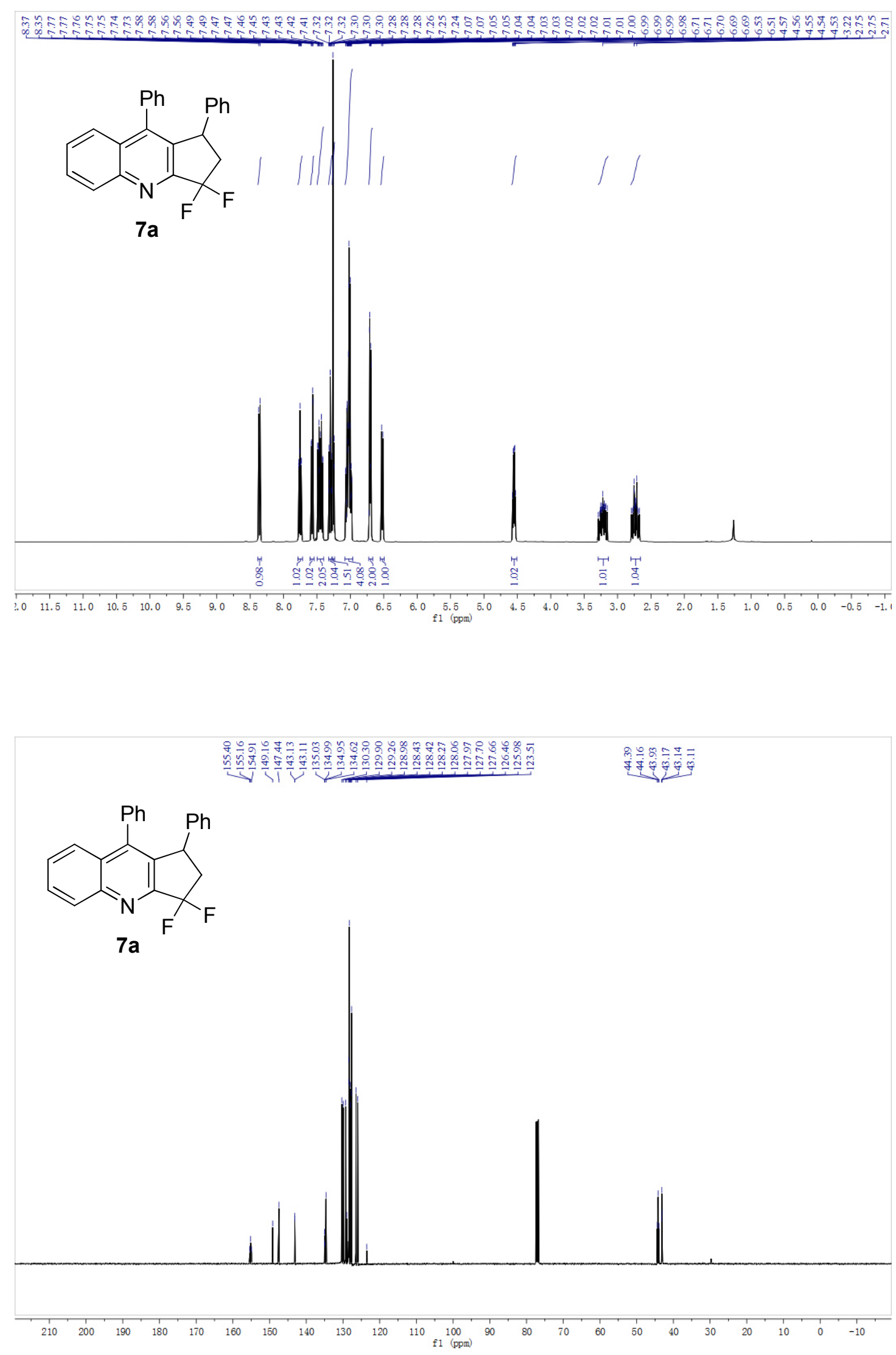


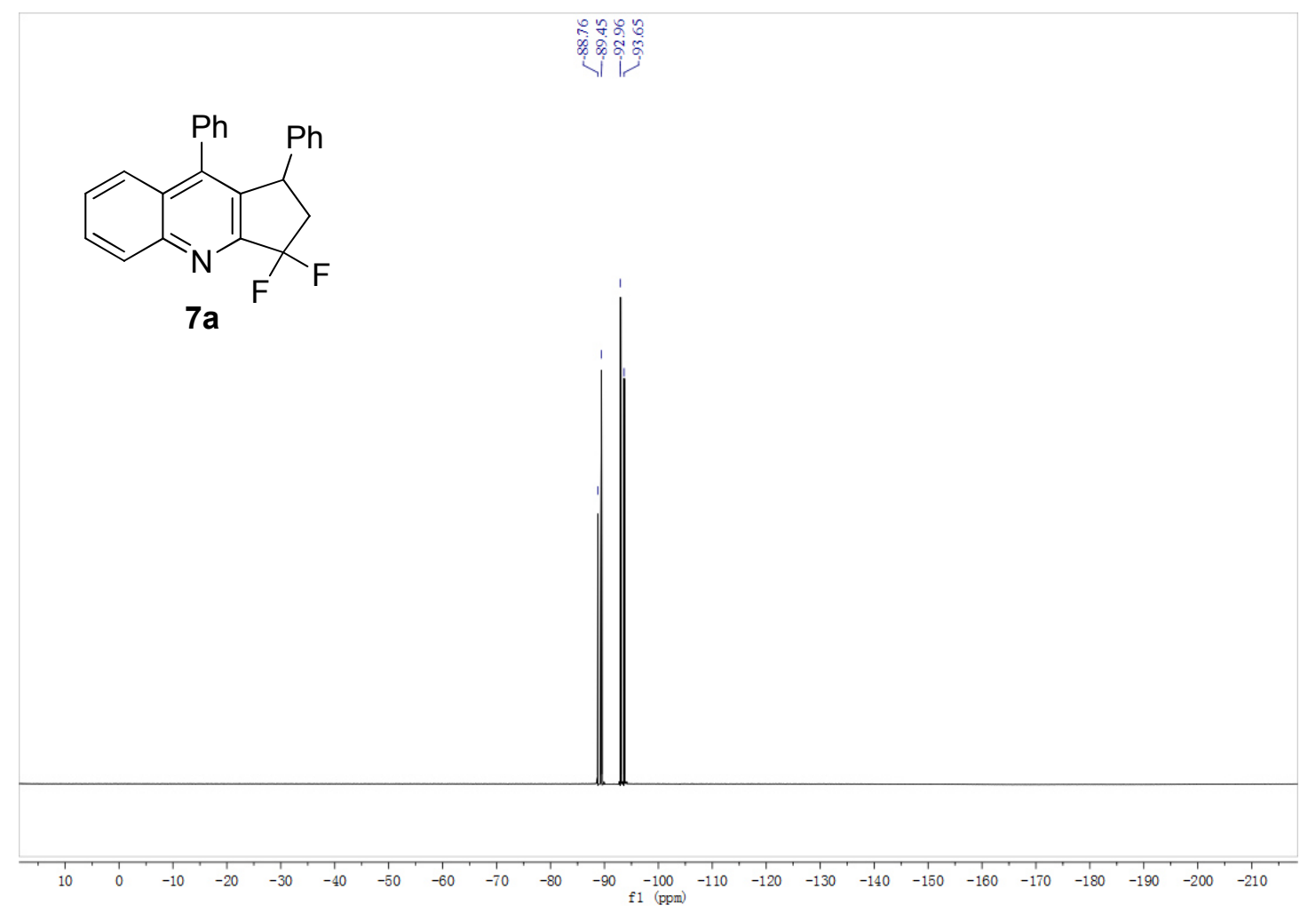

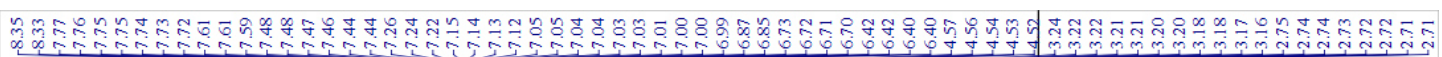<smiles>C#CC(C)c1ccc(C)cc1</smiles>

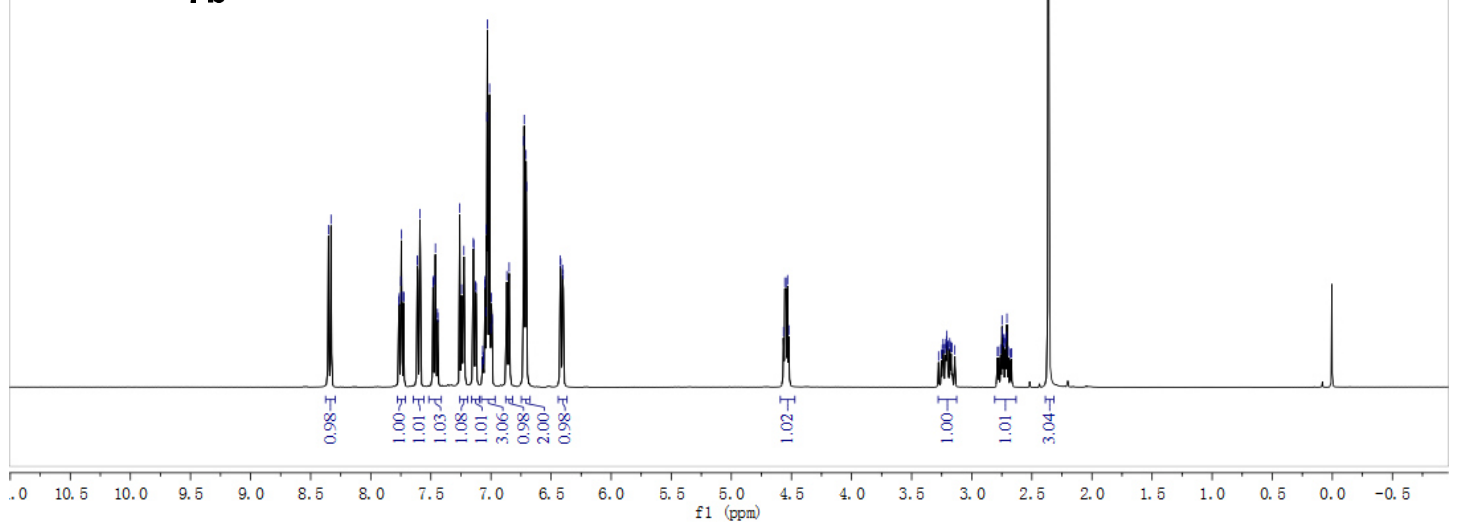




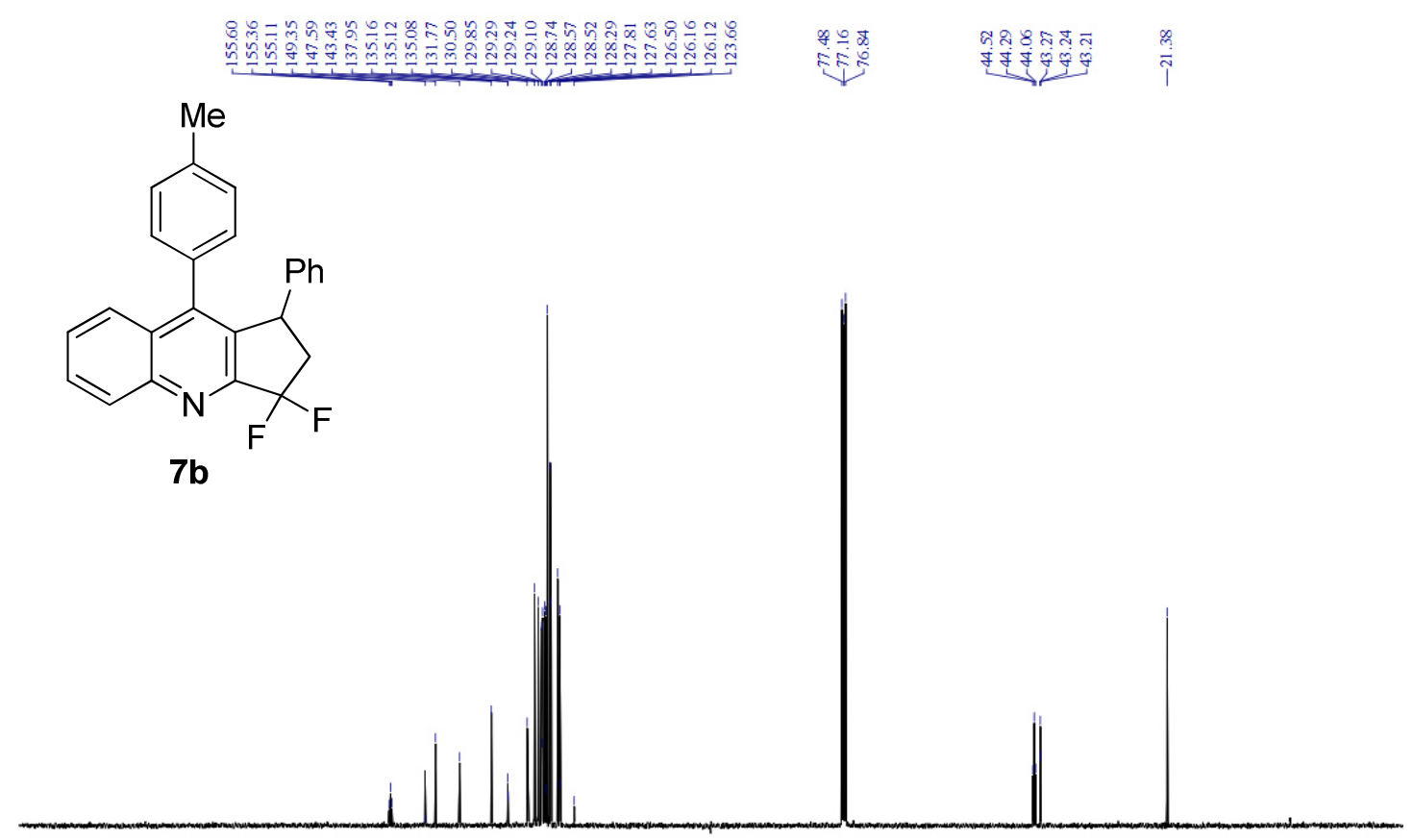

$\begin{array}{lllllllllllllllllllllllllll}1 & 110 & 200 & 190 & 180 & 170 & 160 & 150 & 140 & 130 & 120 & 110 & 100 & 90 & 80 & 70 & 60 & 50 & 40 & 30 & 20 & 10 & 0 & -10\end{array}$

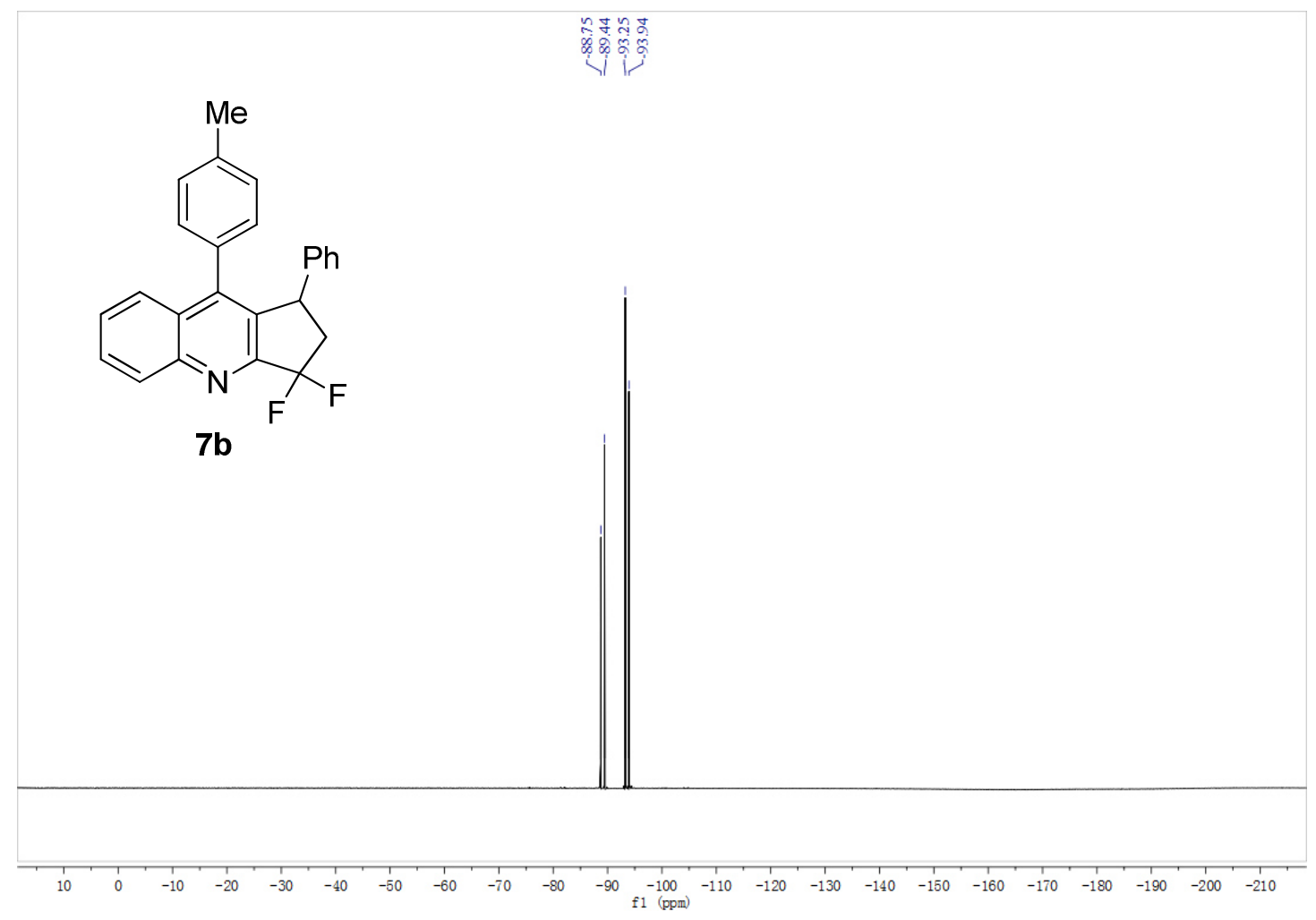




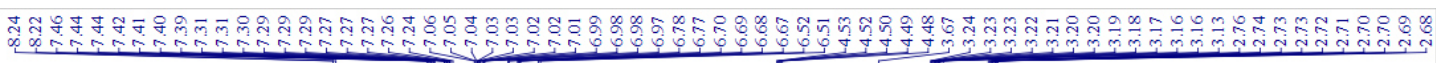<smiles>COc1ccc(-c2c3c(nc4ccccc24)C(F)(F)CC3c2ccccc2)cc1</smiles>
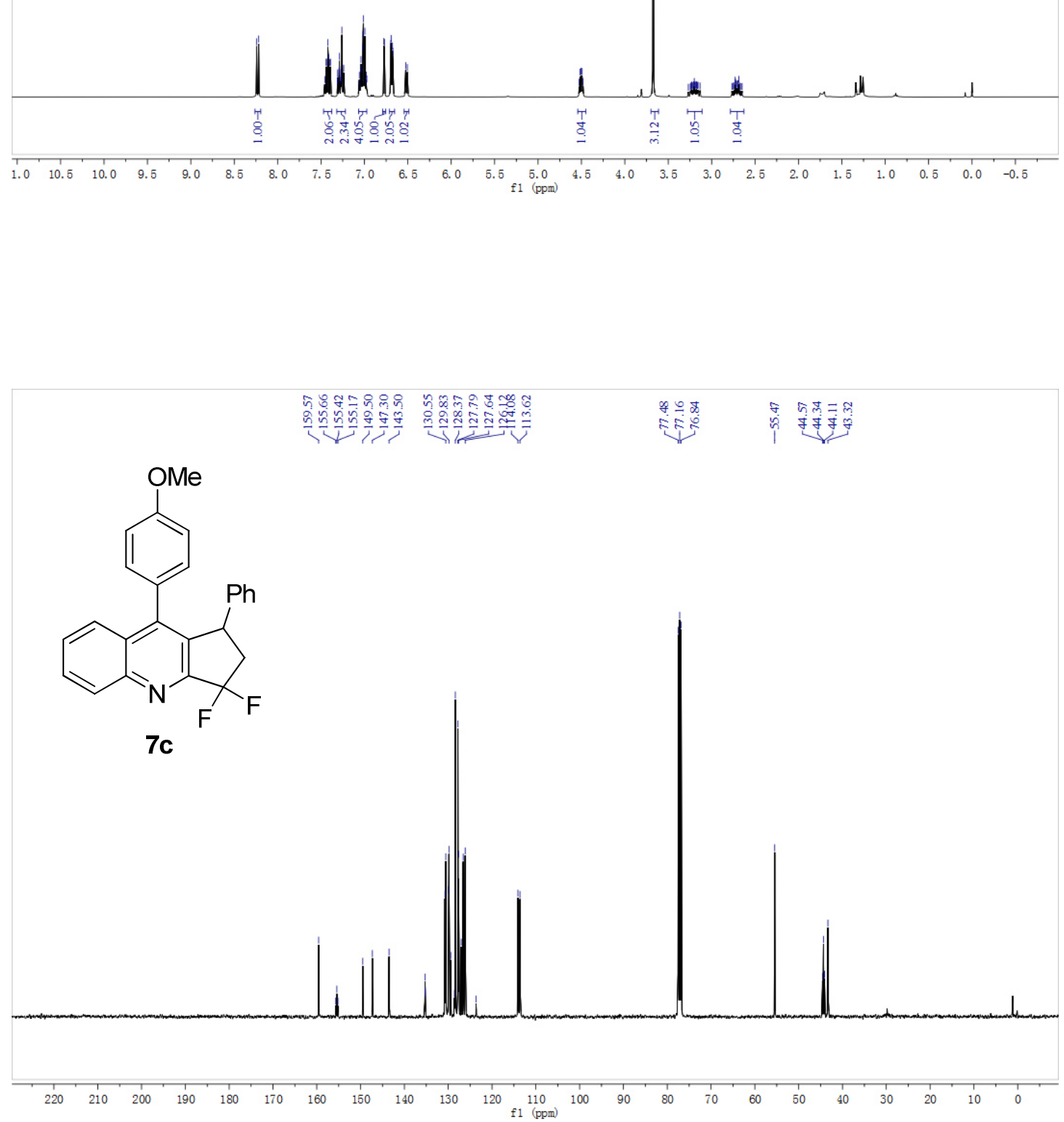


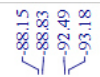<smiles>COc1ccc(-c2c3c(nc4ccccc24)C(F)(F)CC3c2ccccc2)cc1</smiles>

7c

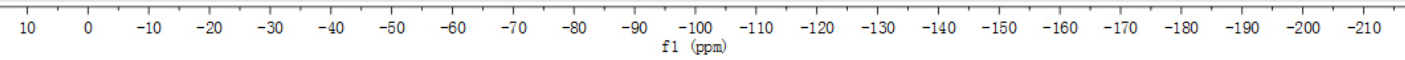

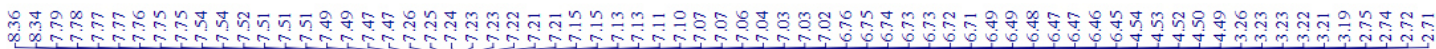

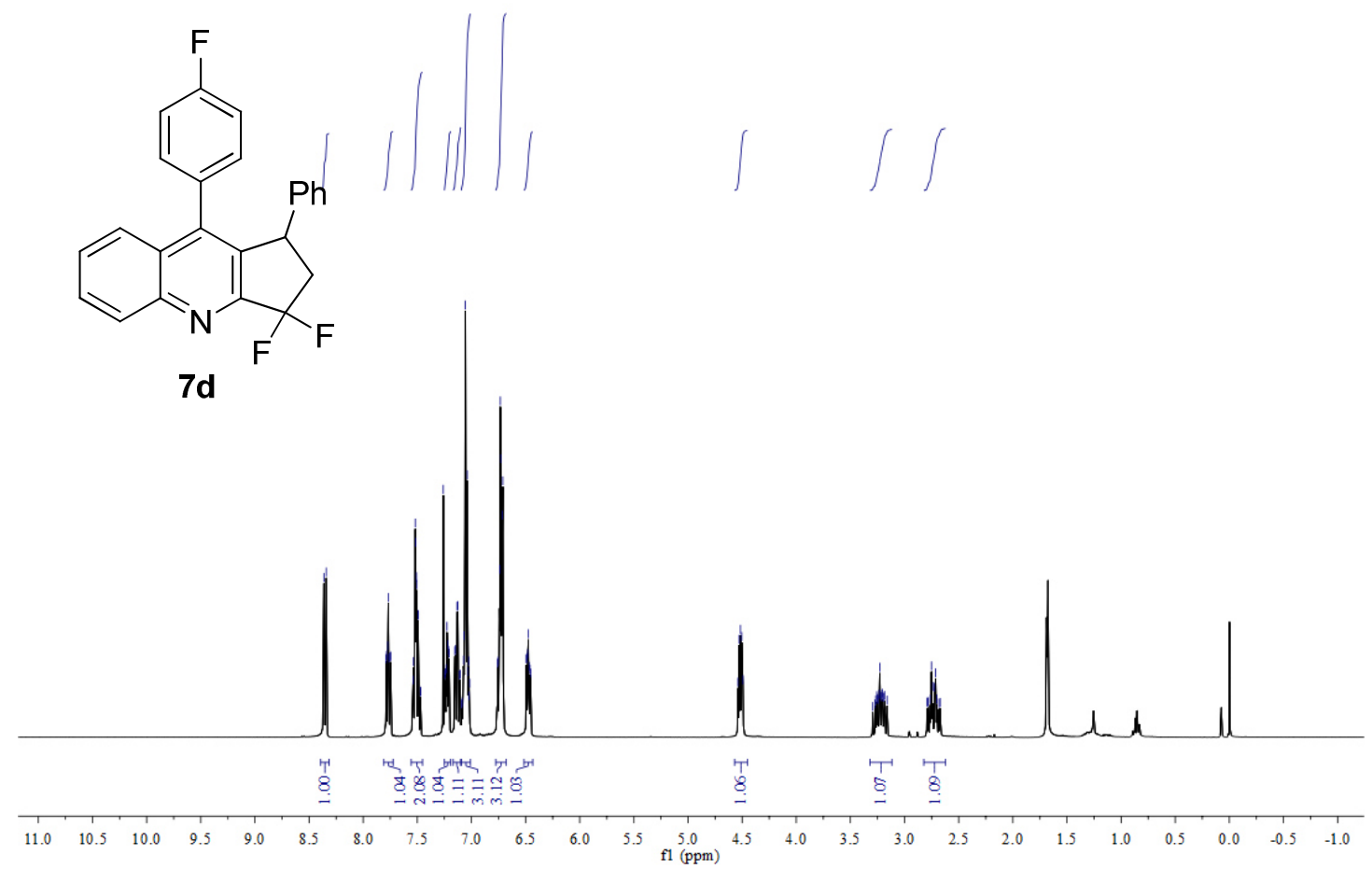



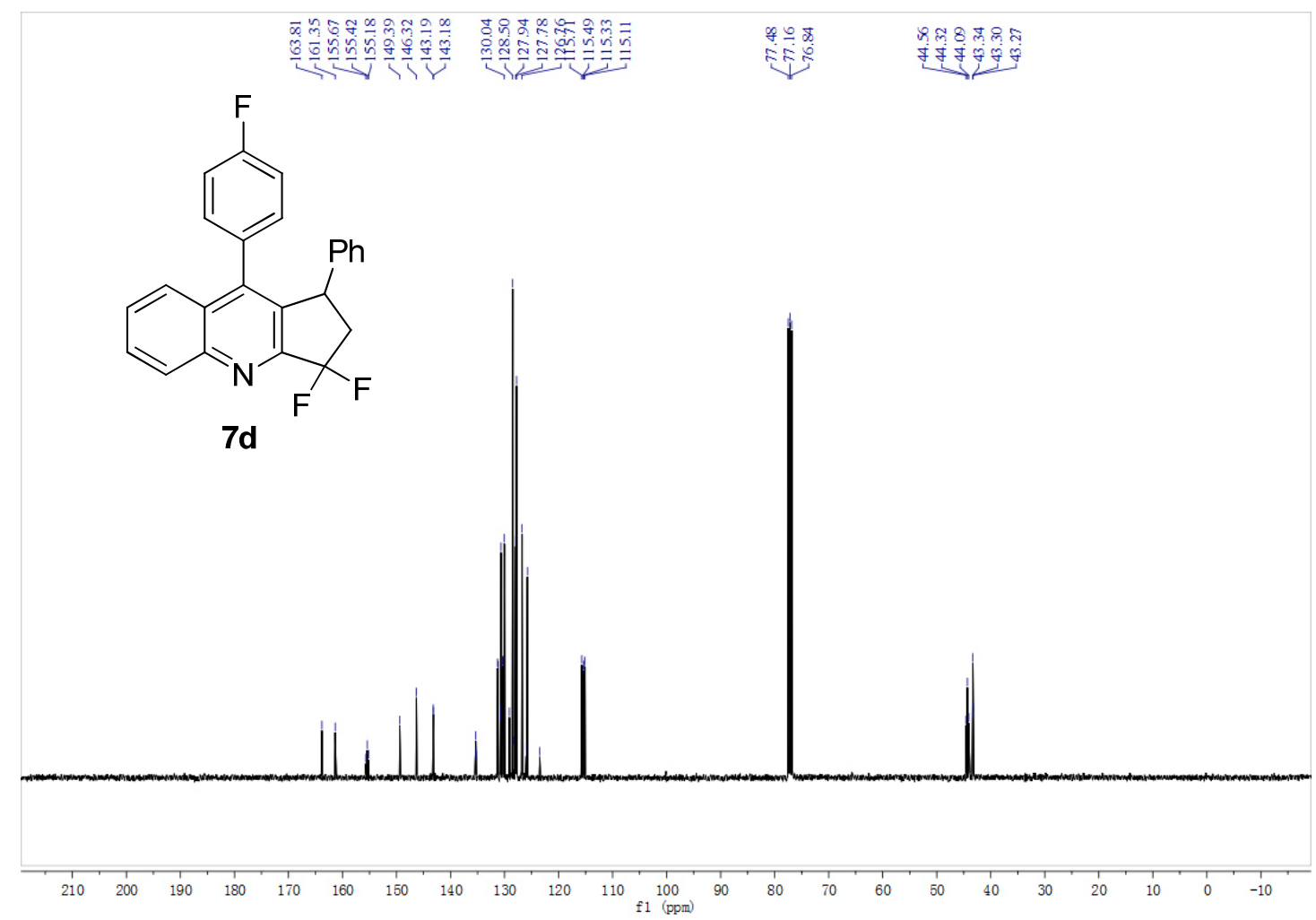

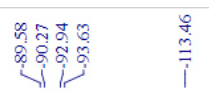<smiles>Fc1ccc(-c2c3c(nc4ccccc24)C(F)(F)CC3c2ccccc2)cc1</smiles>

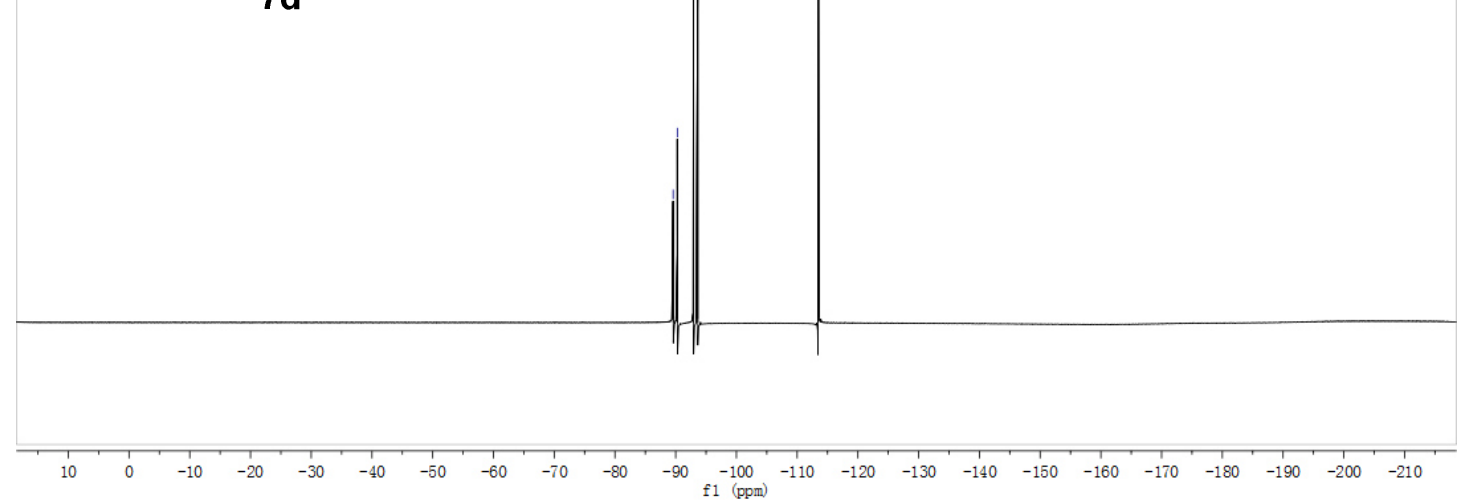



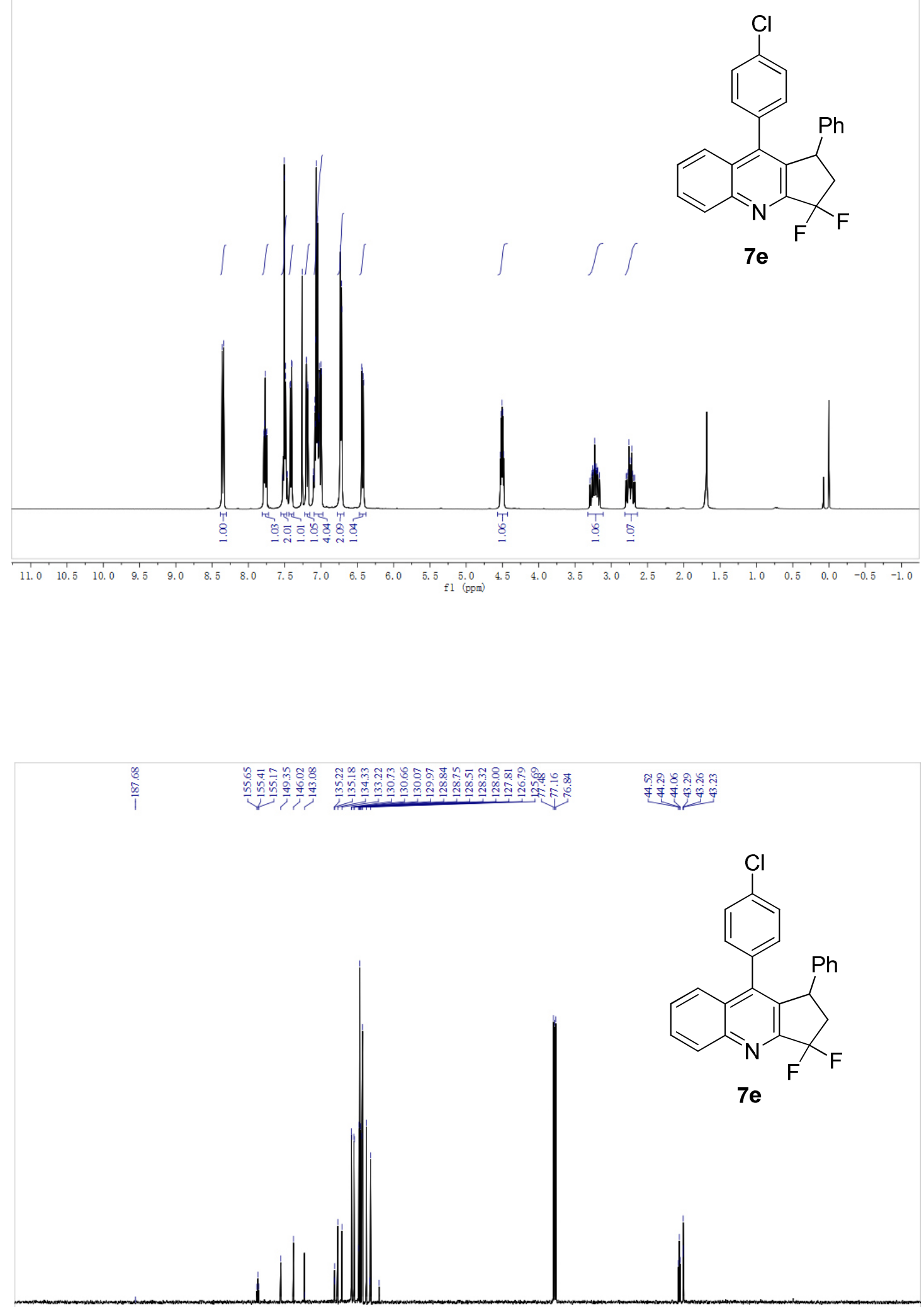

$\begin{array}{lllllllllllllllllllllllllll}1 & 1 & 200 & 190 & 180 & 170 & 160 & 150 & 140 & 130 & 120 & 110 & 100 & 90 & 80 & 70 & 60 & 50 & 40 & 30 & 20 & 10 & 0 & -10\end{array}$ 

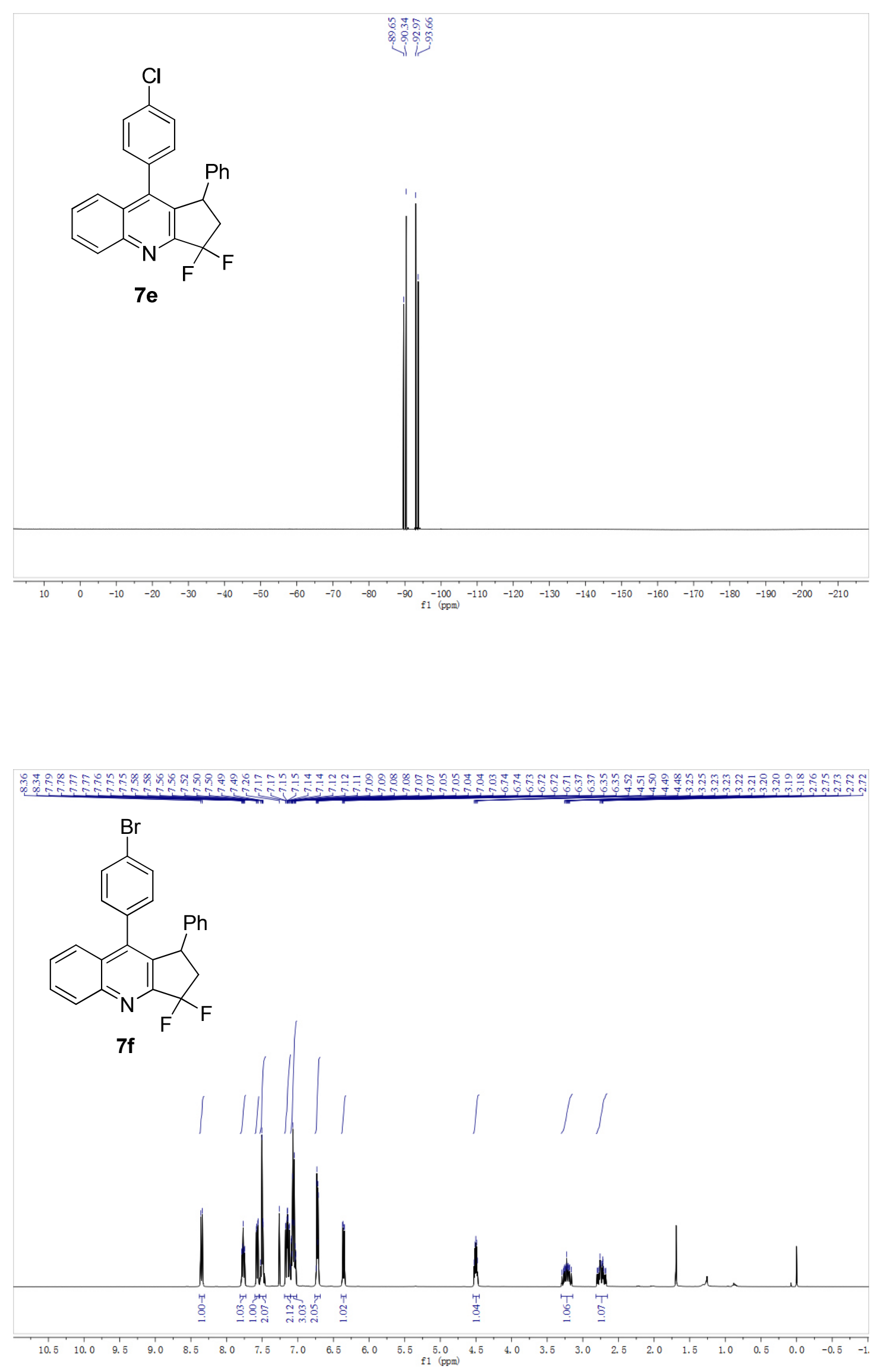

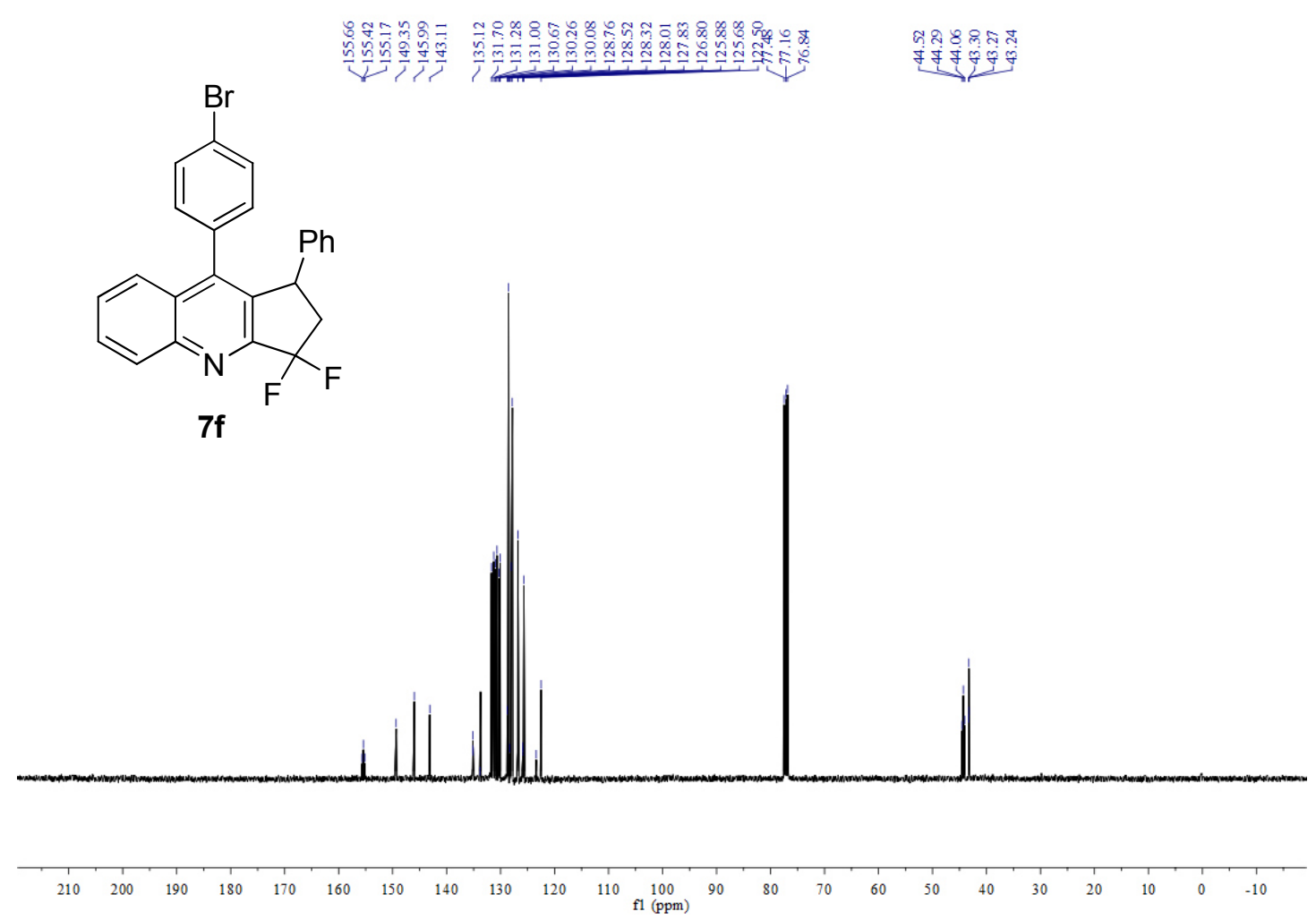<smiles>FC1(F)CC(c2ccccc2)c2c1nc1ccccc1c2-c1ccc(Br)cc1</smiles>

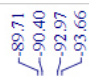

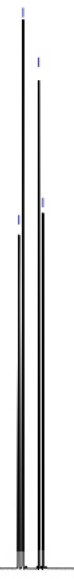

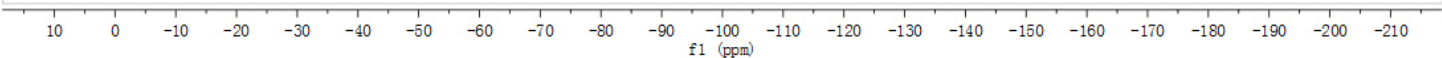




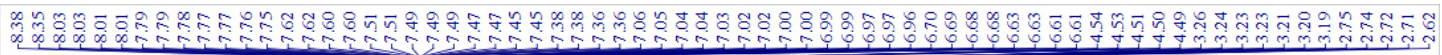<smiles>C#CC1(F)CC(c2ccccc2)c2c1nc1ccccc1c2-c1ccc(C(C)=O)cc1</smiles>

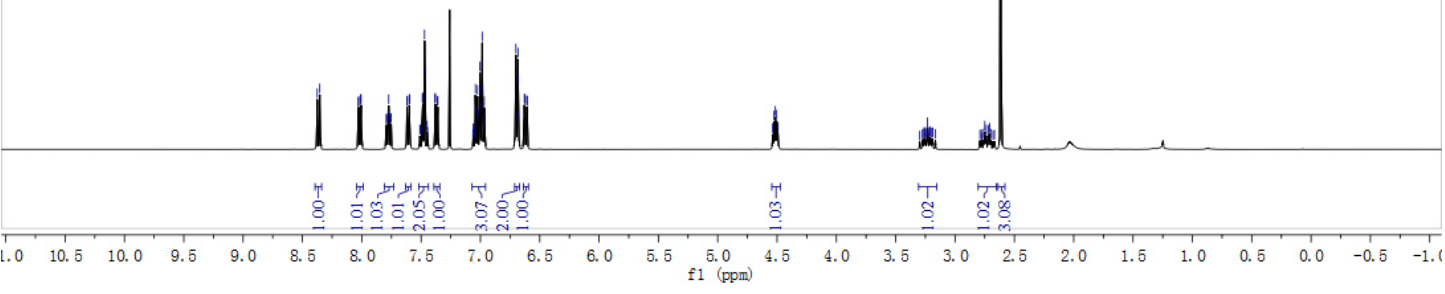

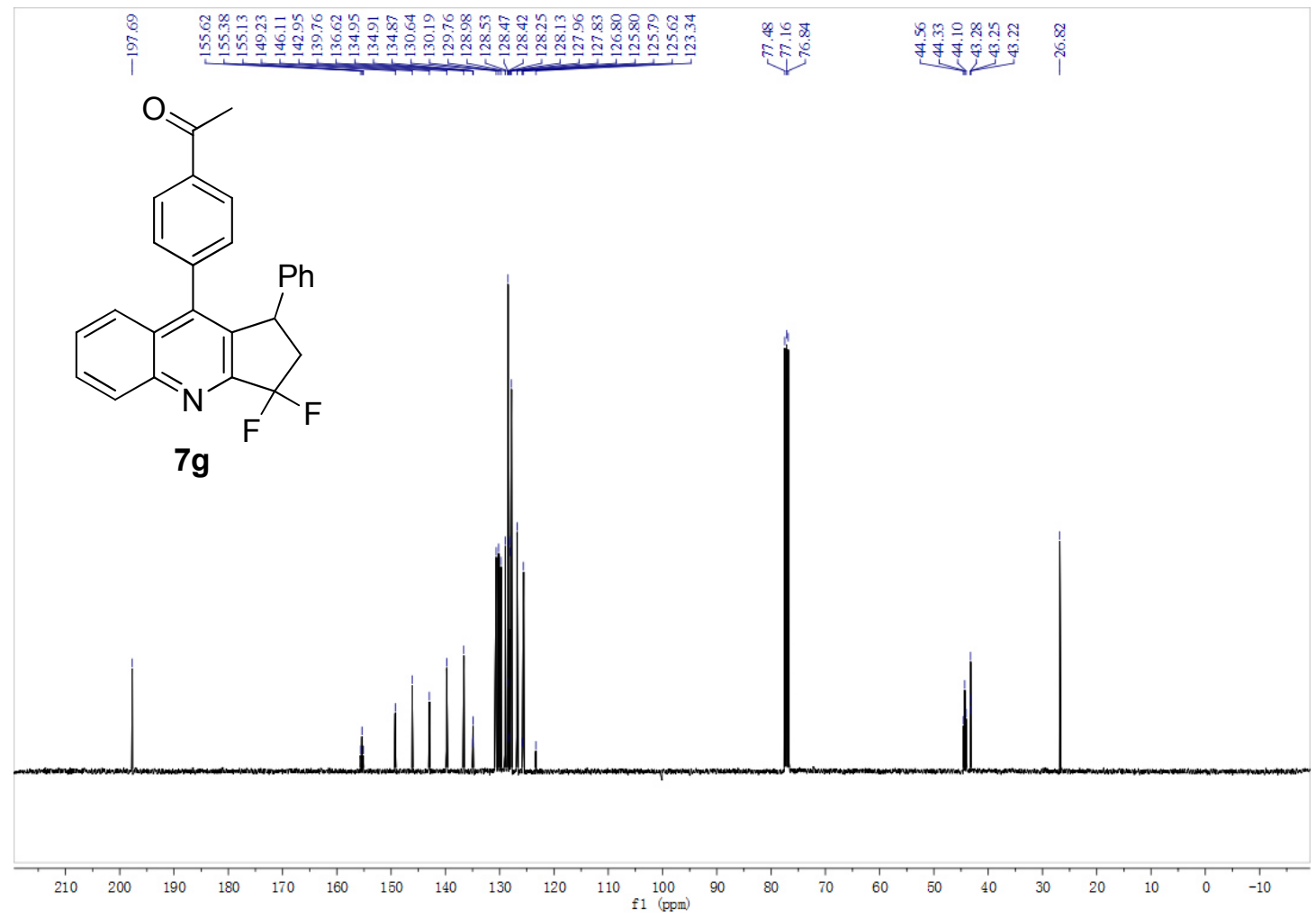




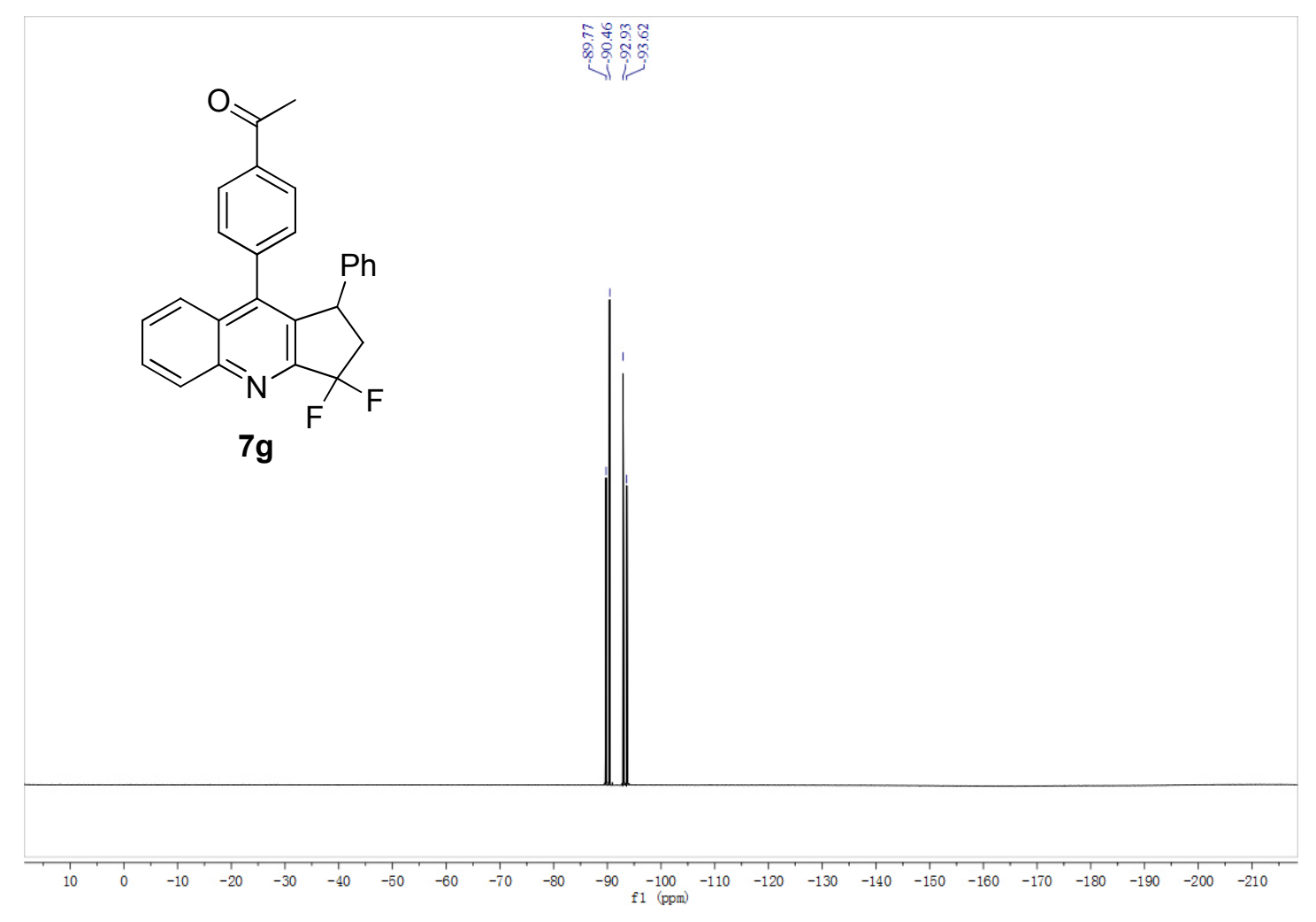

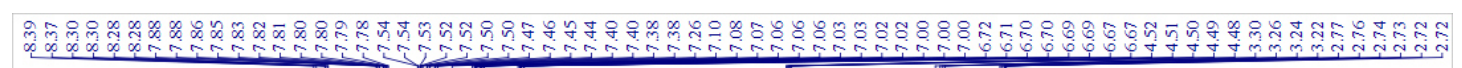

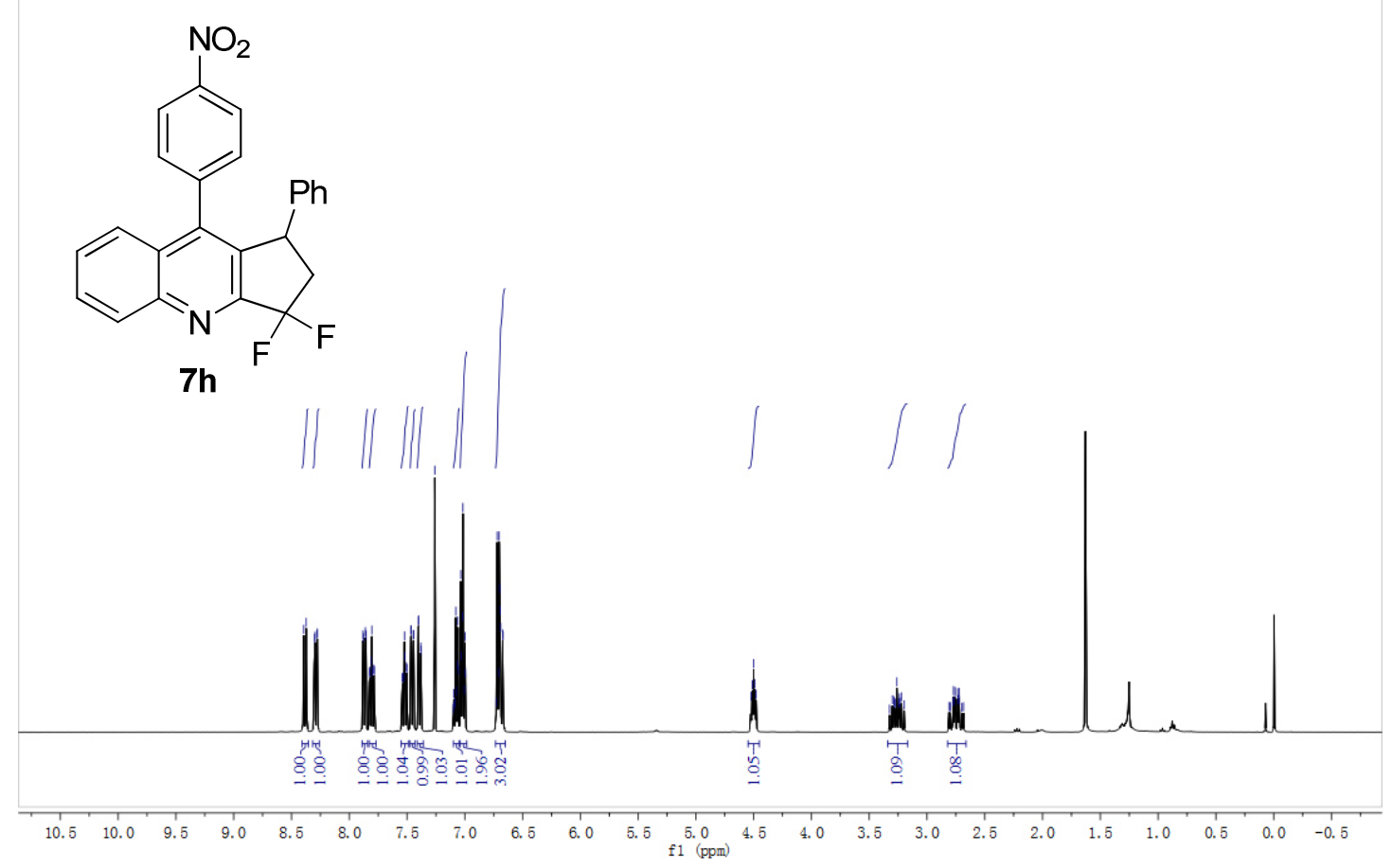



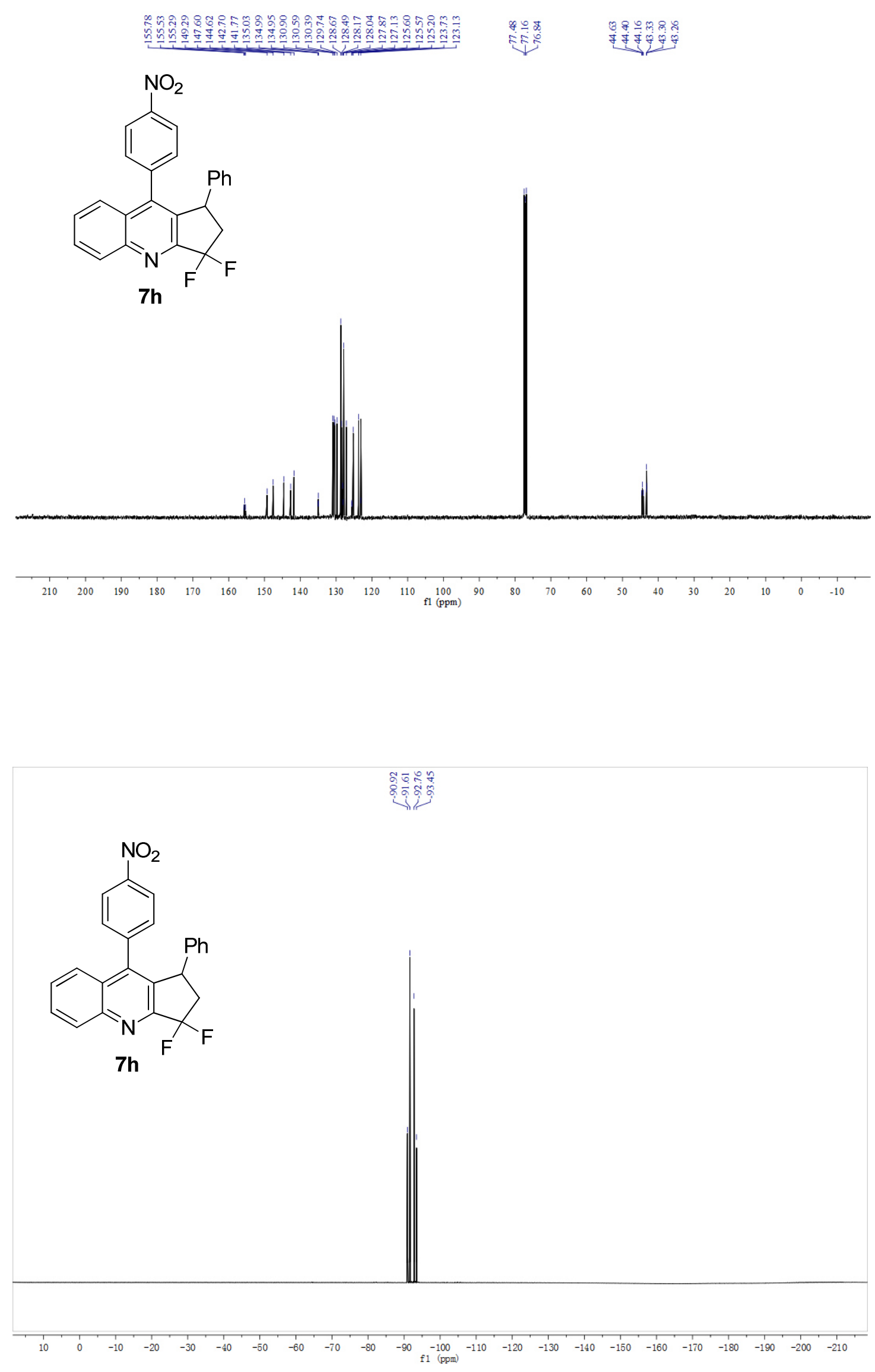

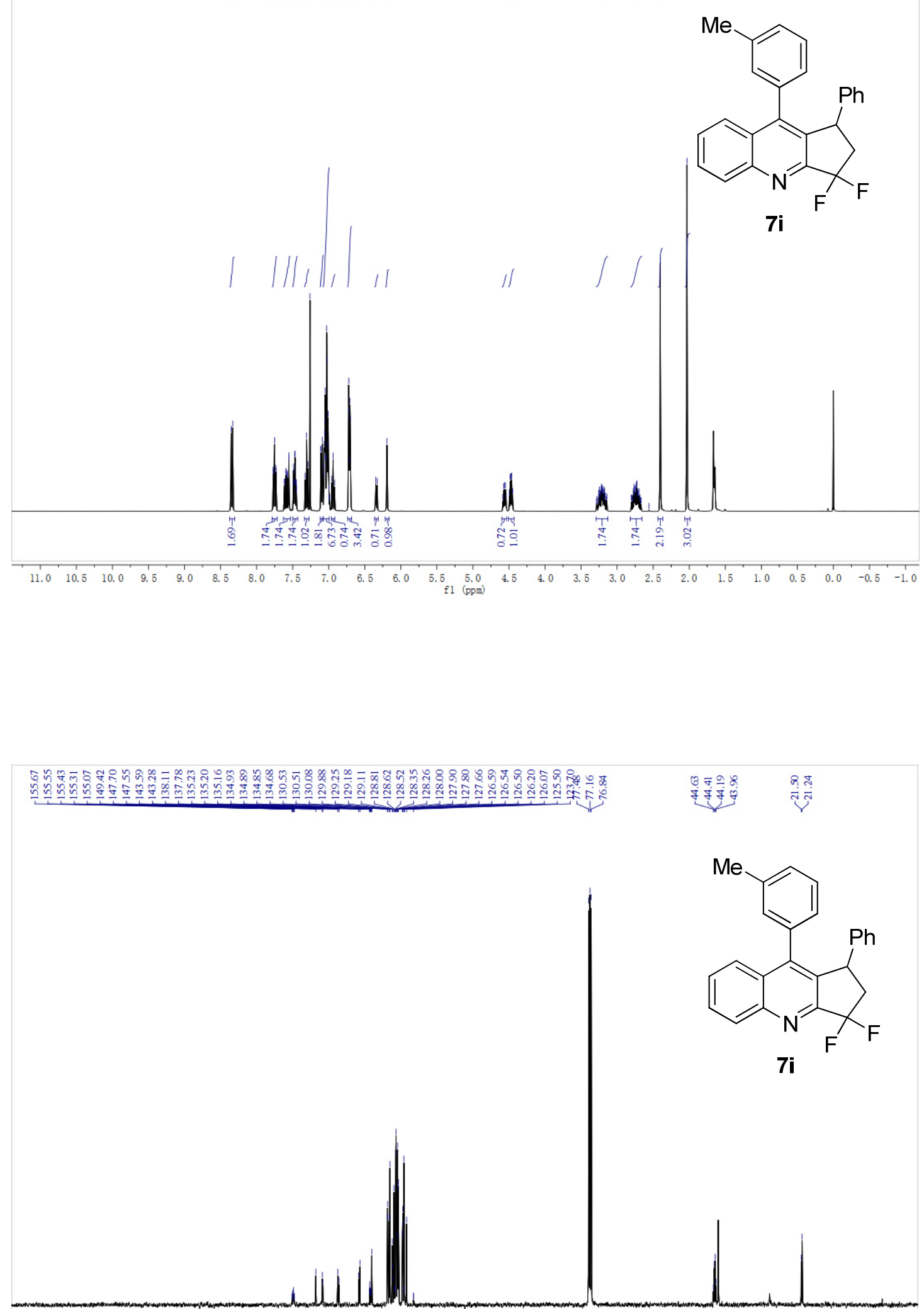

$220 \quad 210 \quad 200 \quad 190 \quad 180 \quad 170 \quad 160 \quad 150 \quad 140 \quad 130 \quad 120 \underset{f 1\left(\frac{p p m}{110}\right)}{100}$ 


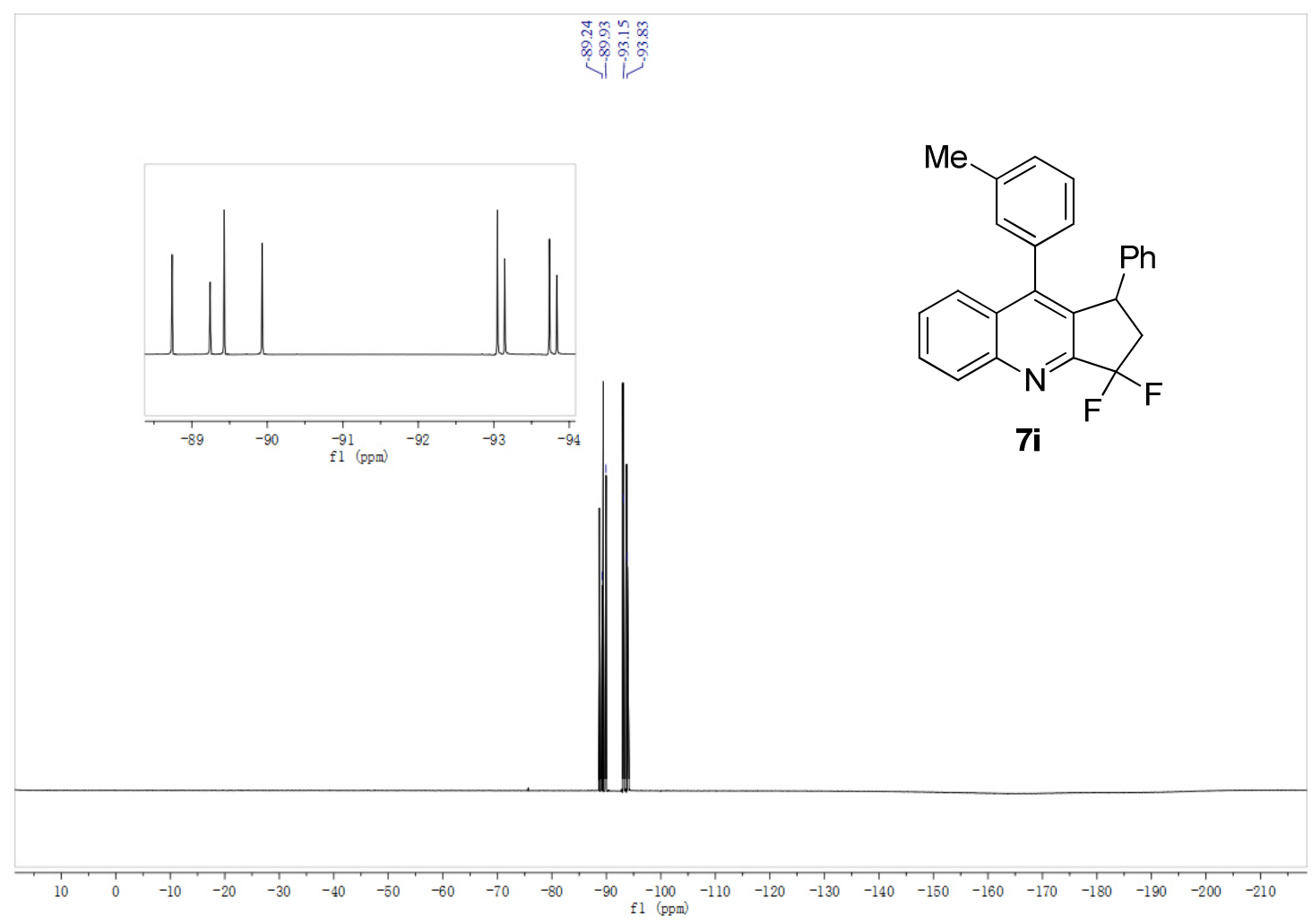

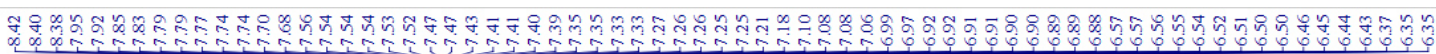

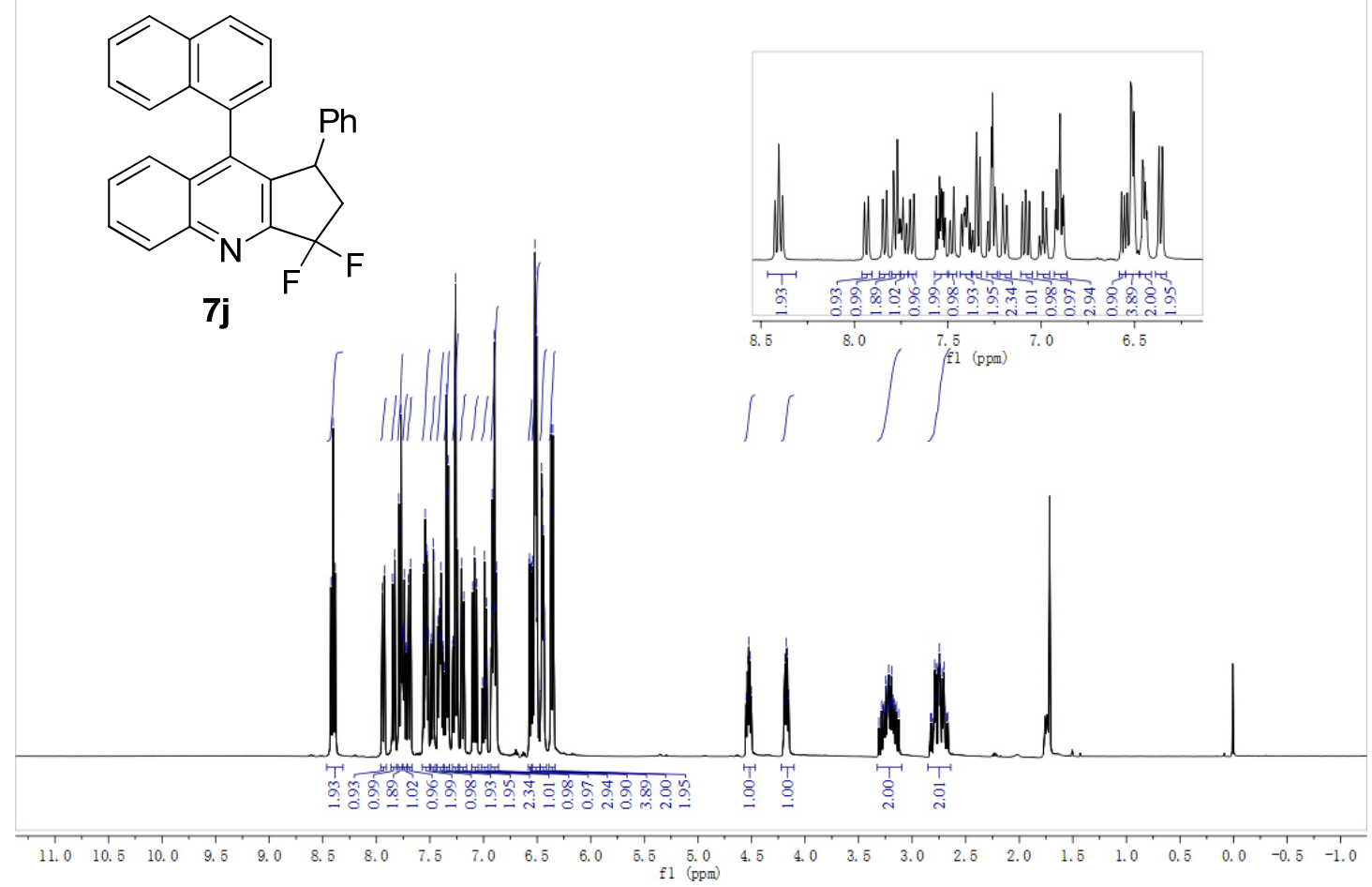



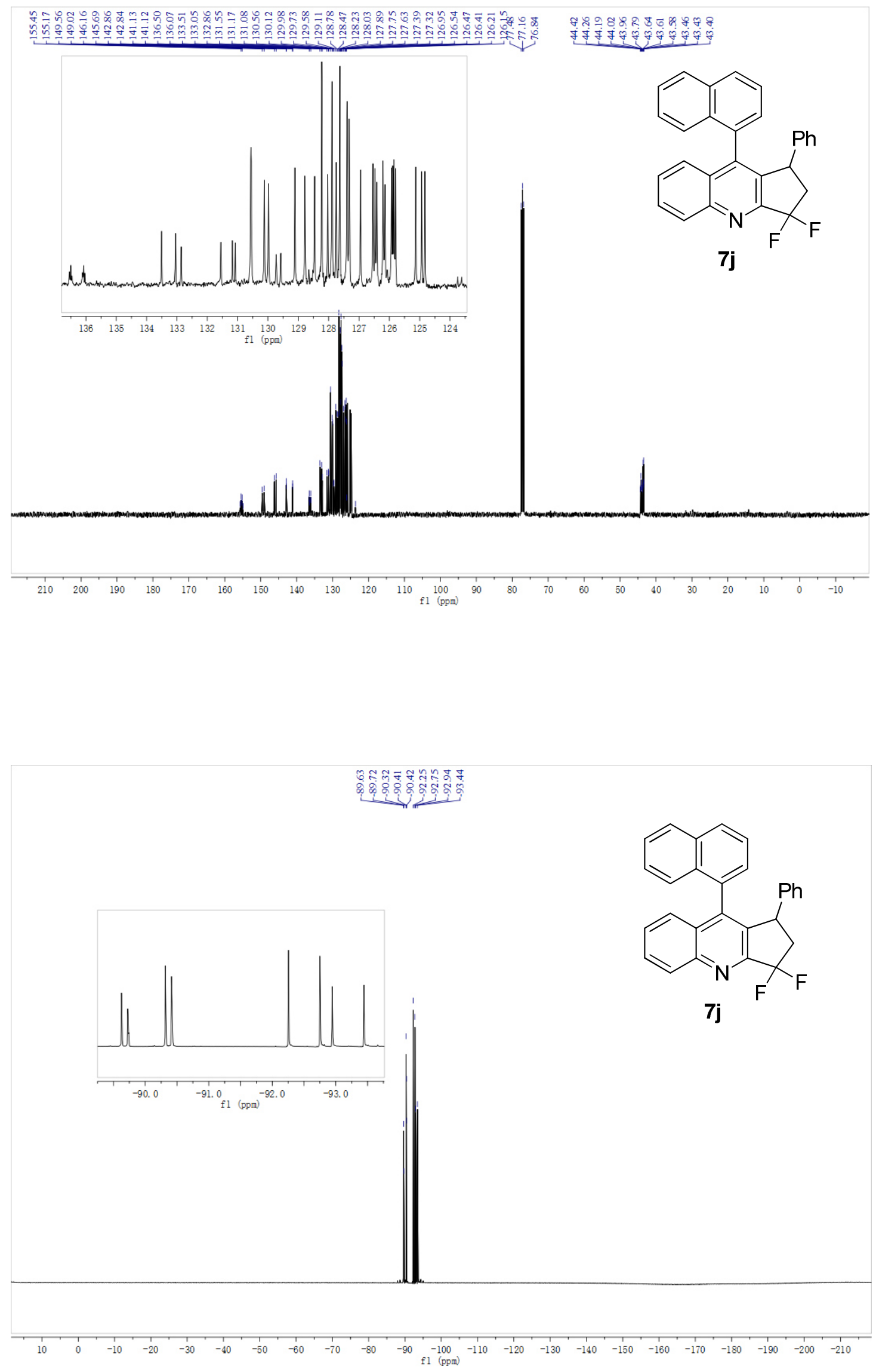

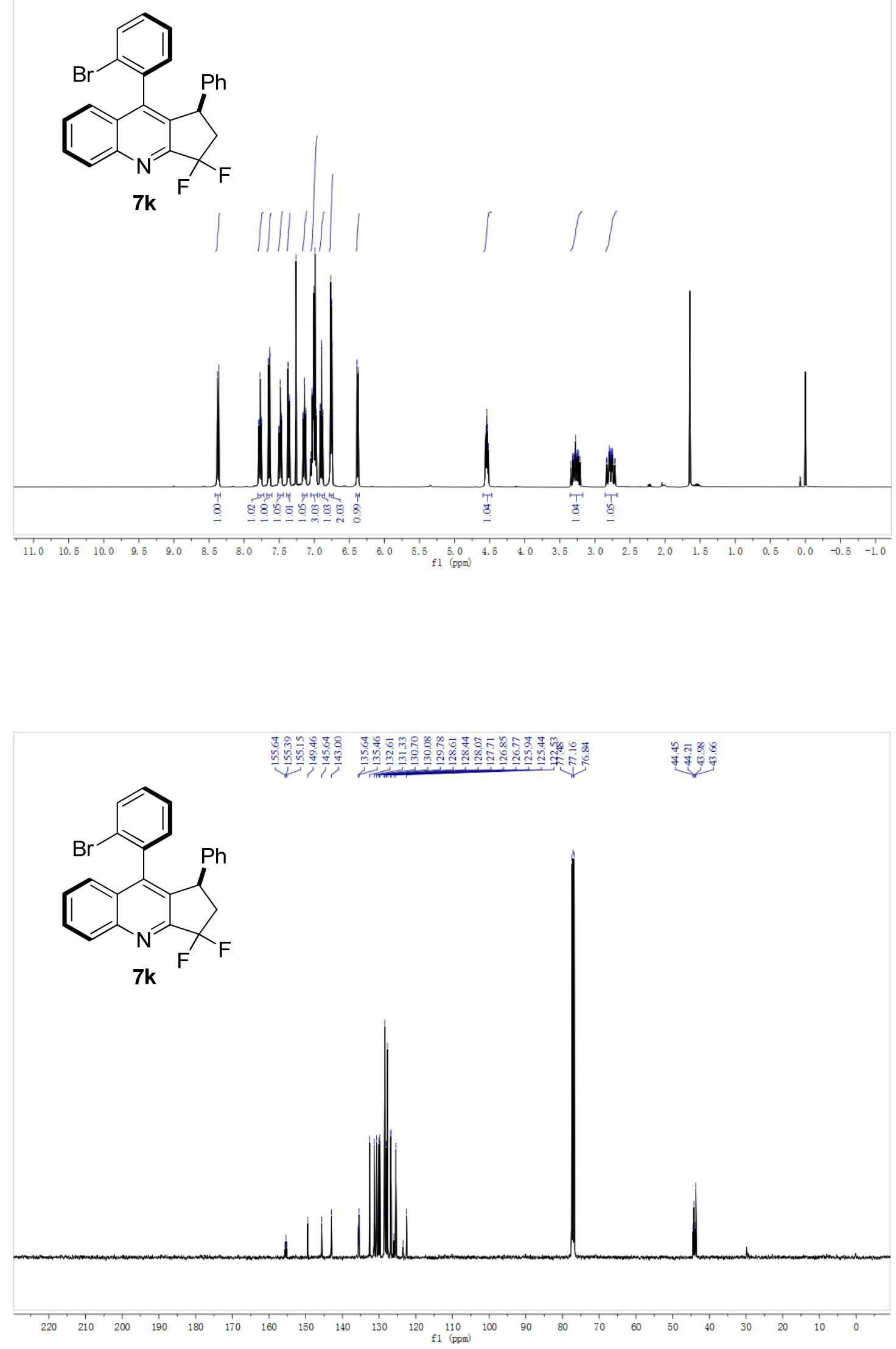


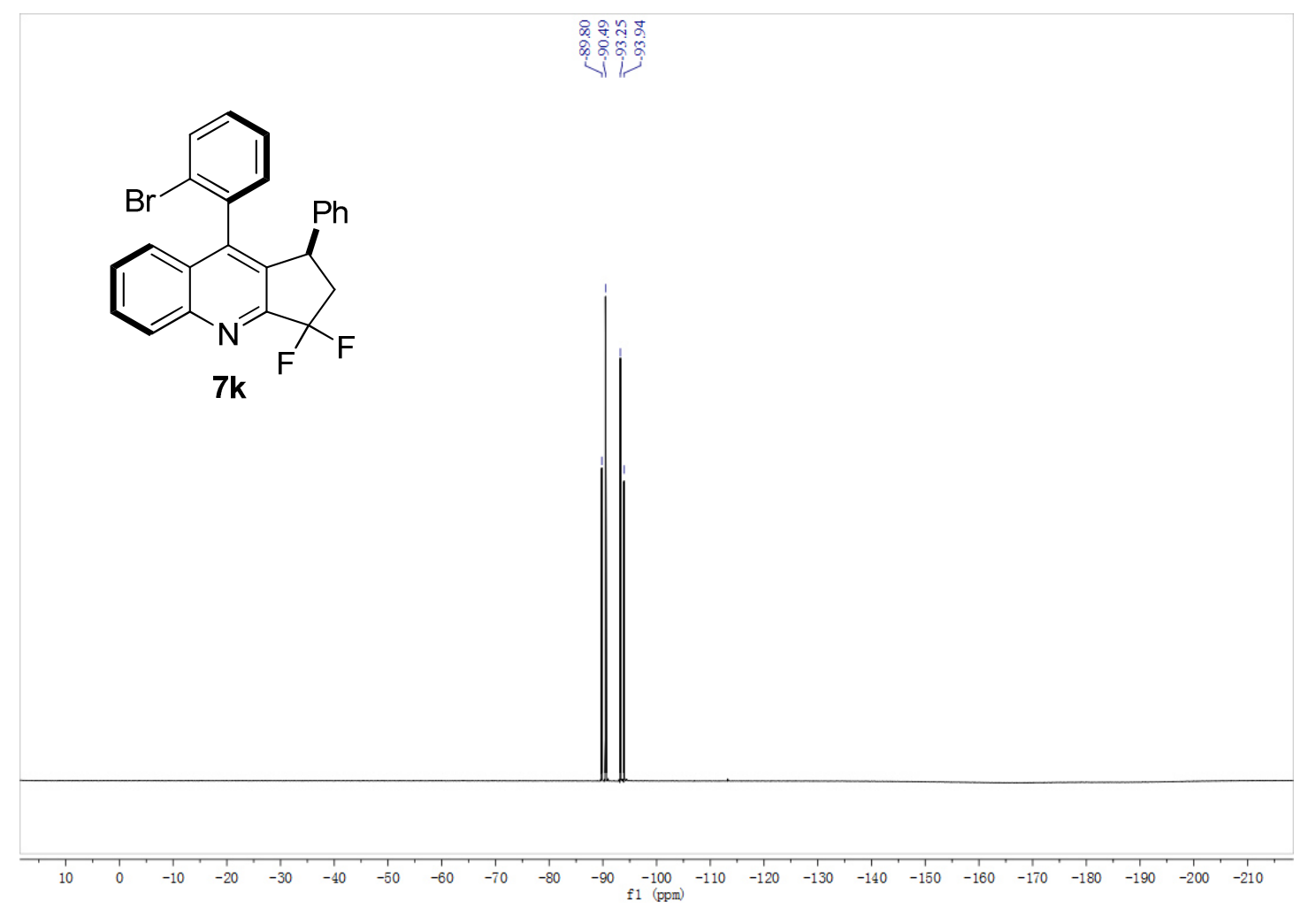

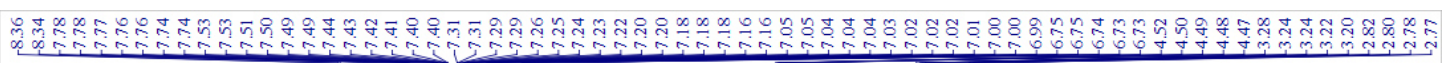

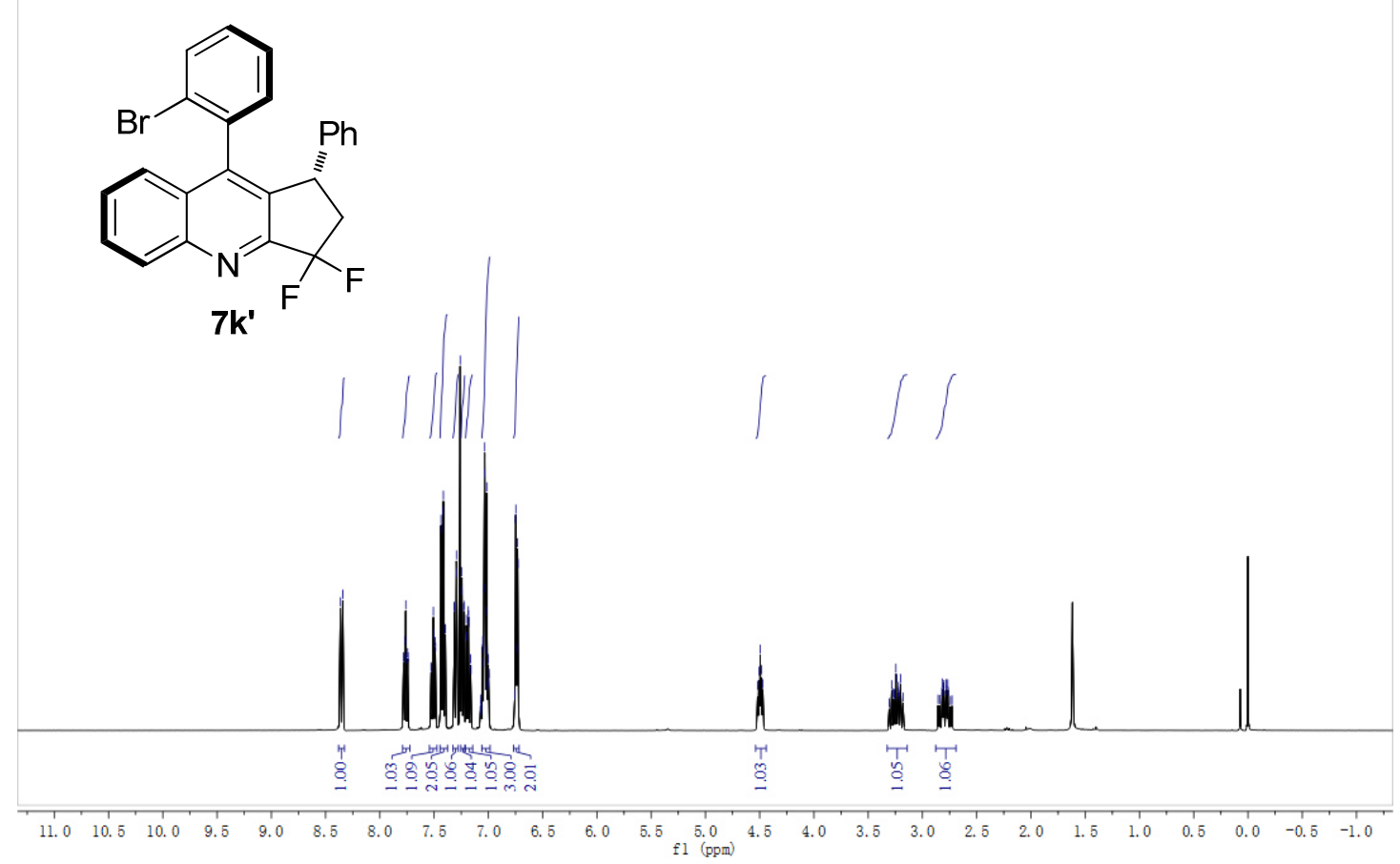




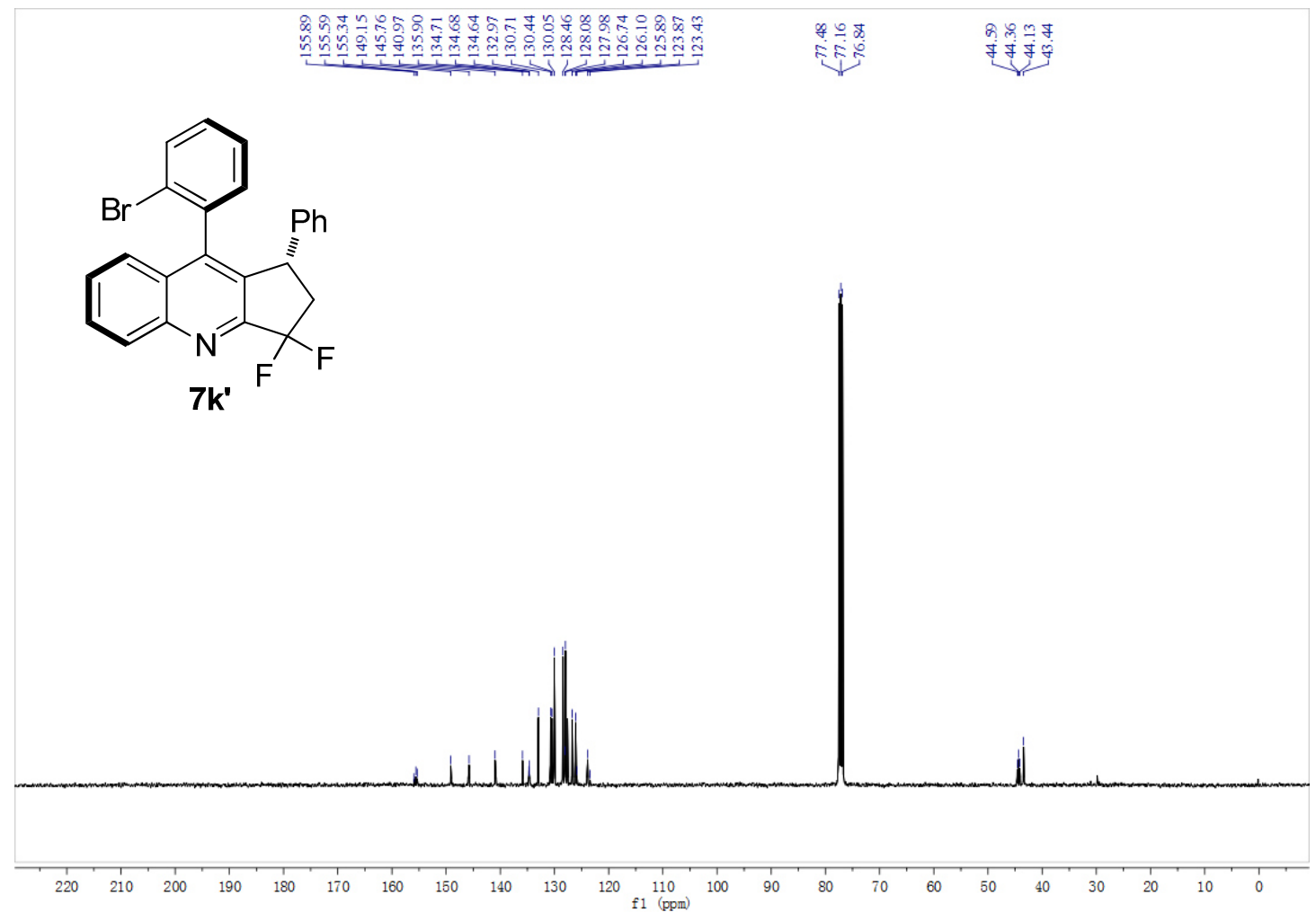

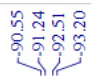

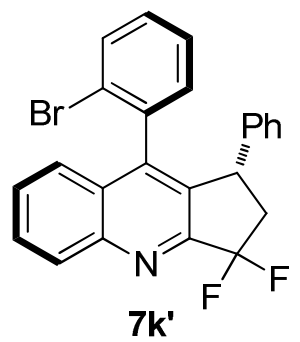



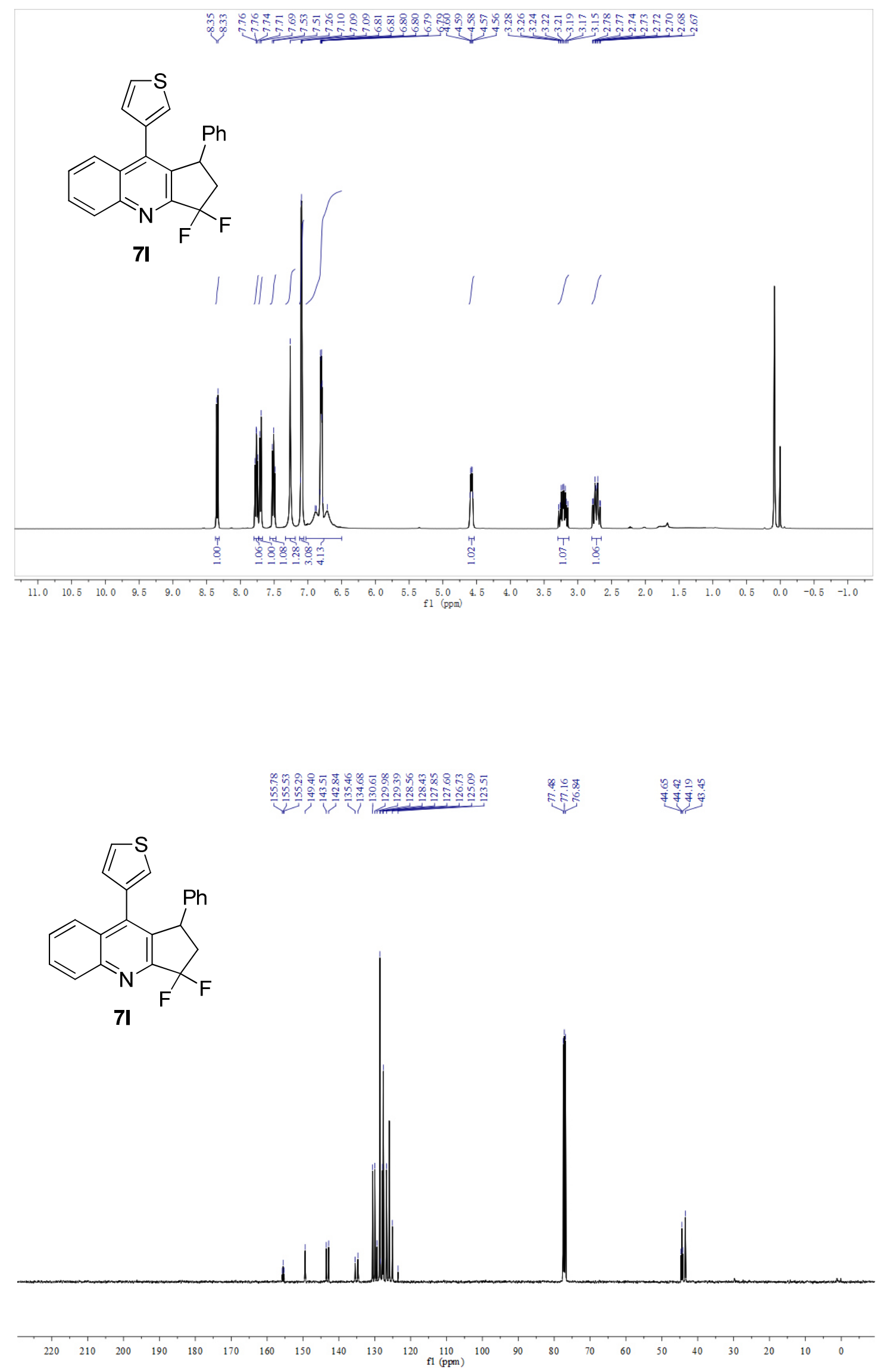


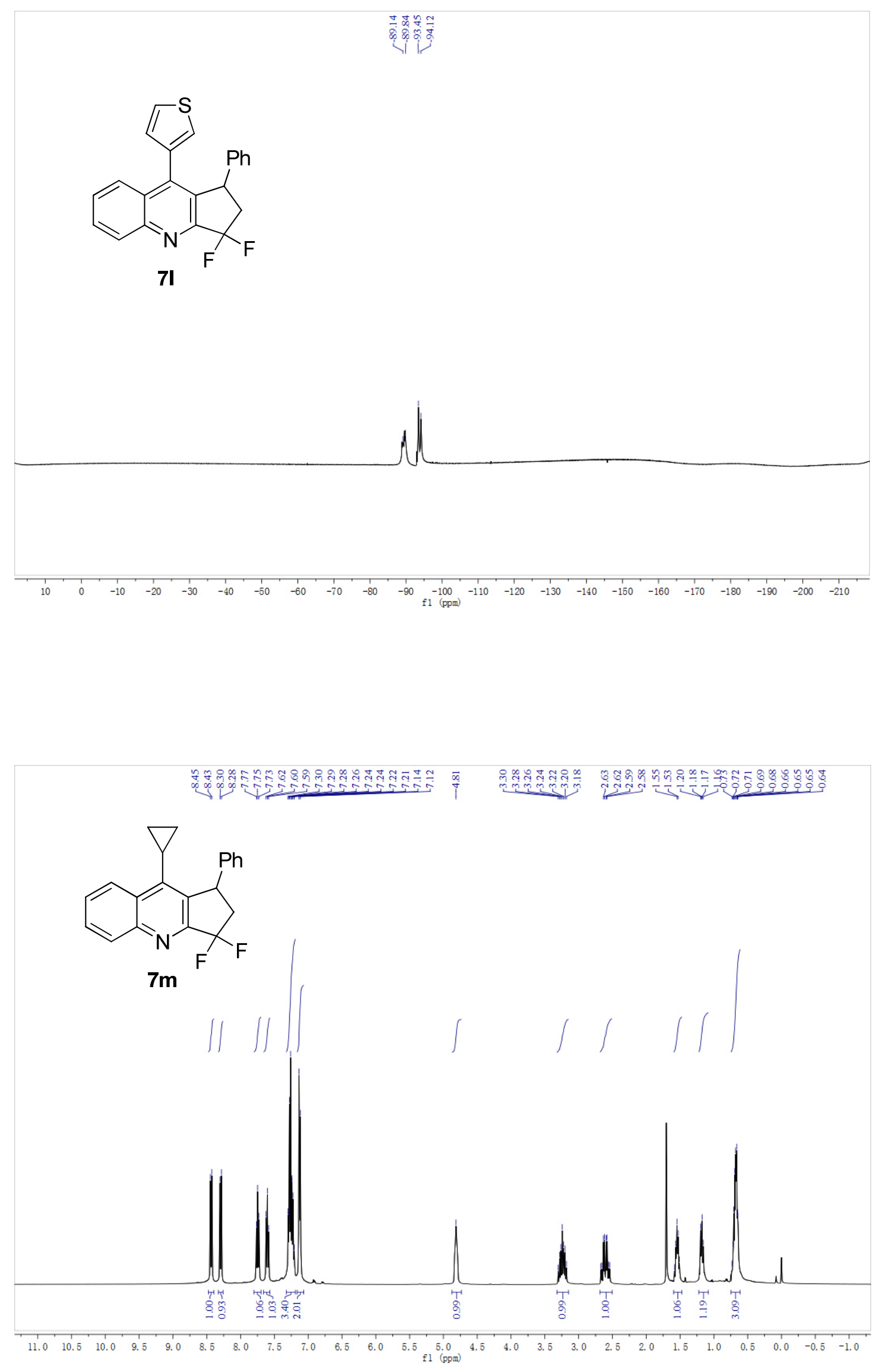




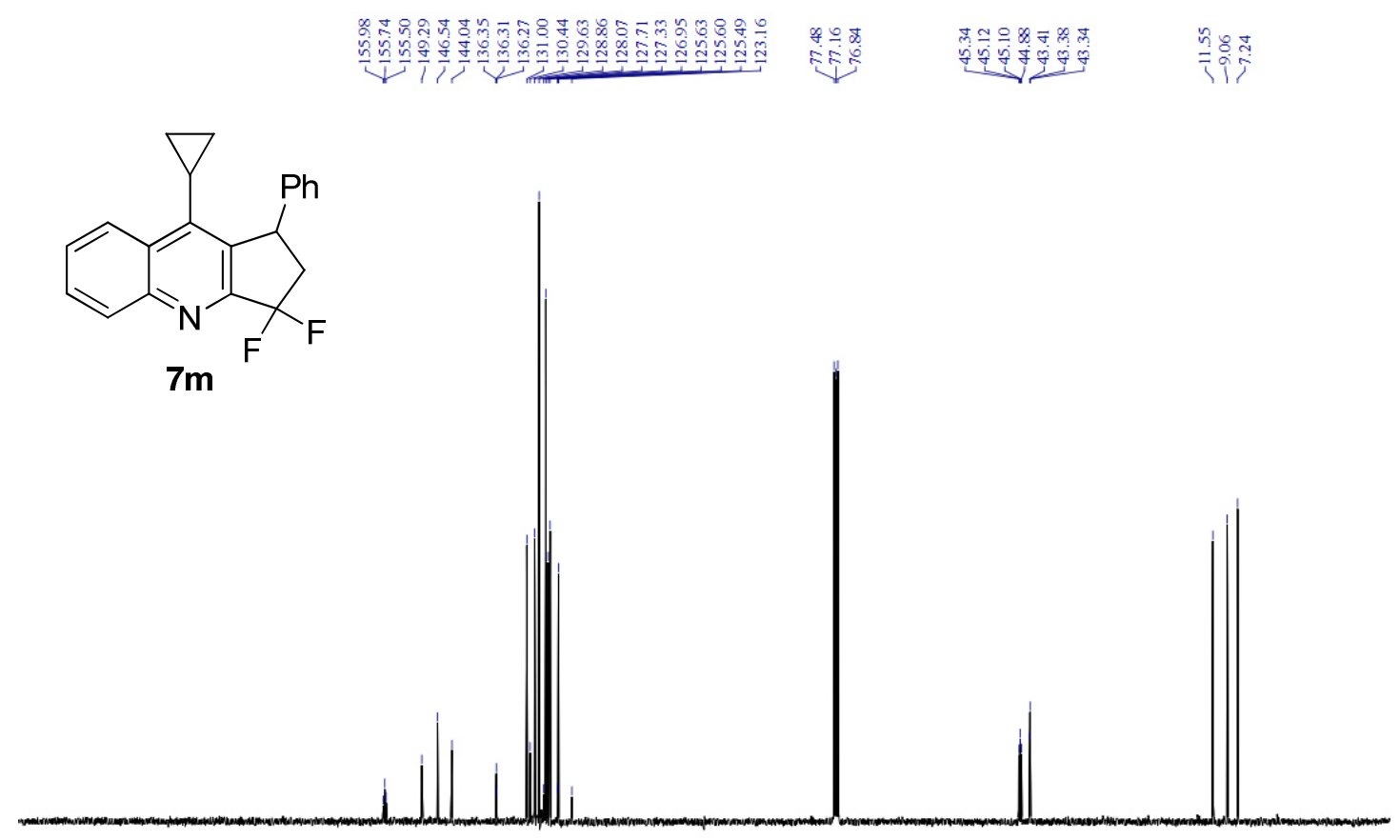

$\begin{array}{lllllllllllllllllllllllllllll}1 & 110 & 200 & 190 & 180 & 170 & 160 & 150 & 140 & 130 & 120 & 110 & \begin{array}{c}100 \\ \mathrm{f1}(\mathrm{ppm})\end{array} & 90 & 80 & 70 & 60 & 50 & 40 & 30 & 20 & 10 & 0 & -10\end{array}$

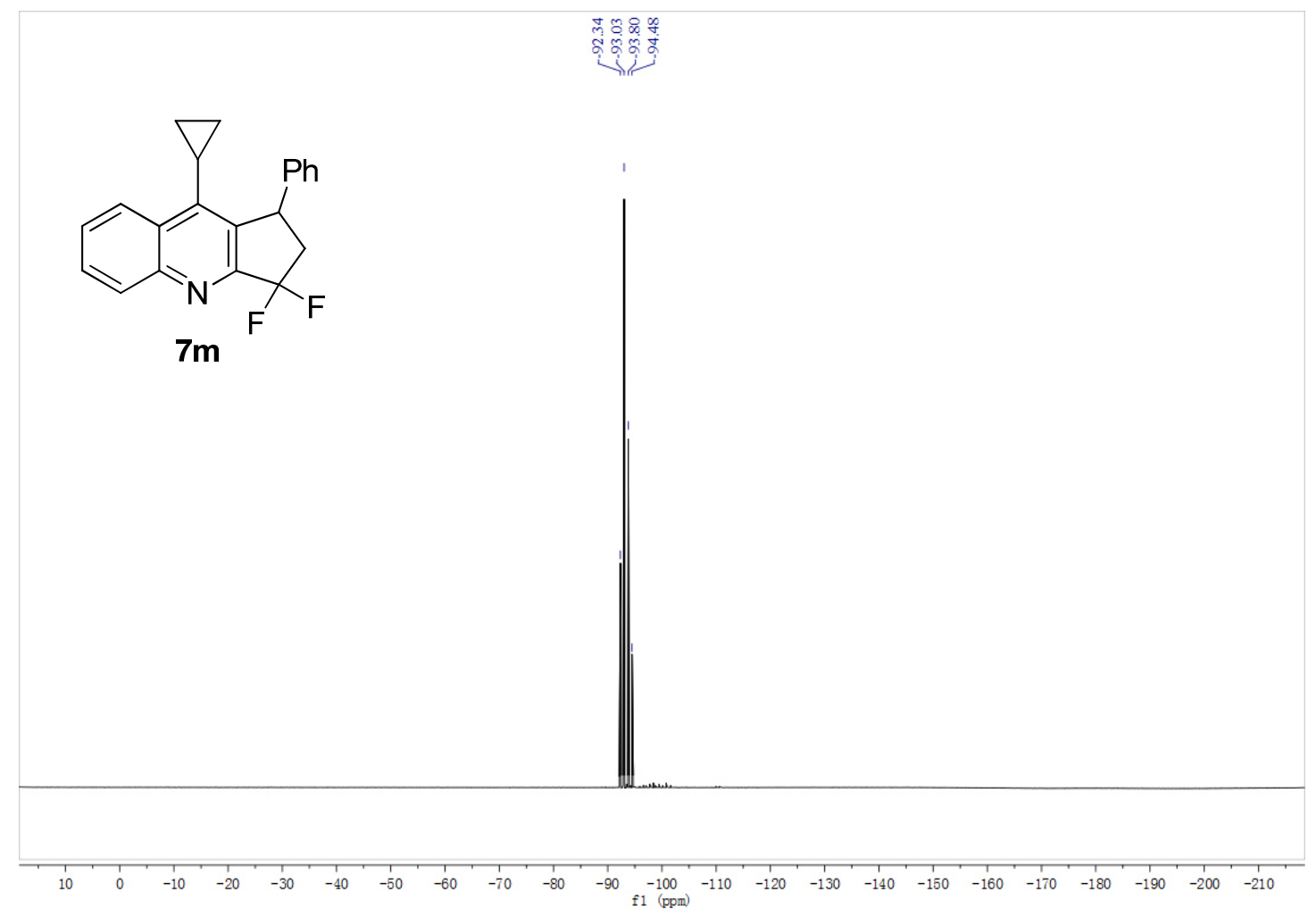



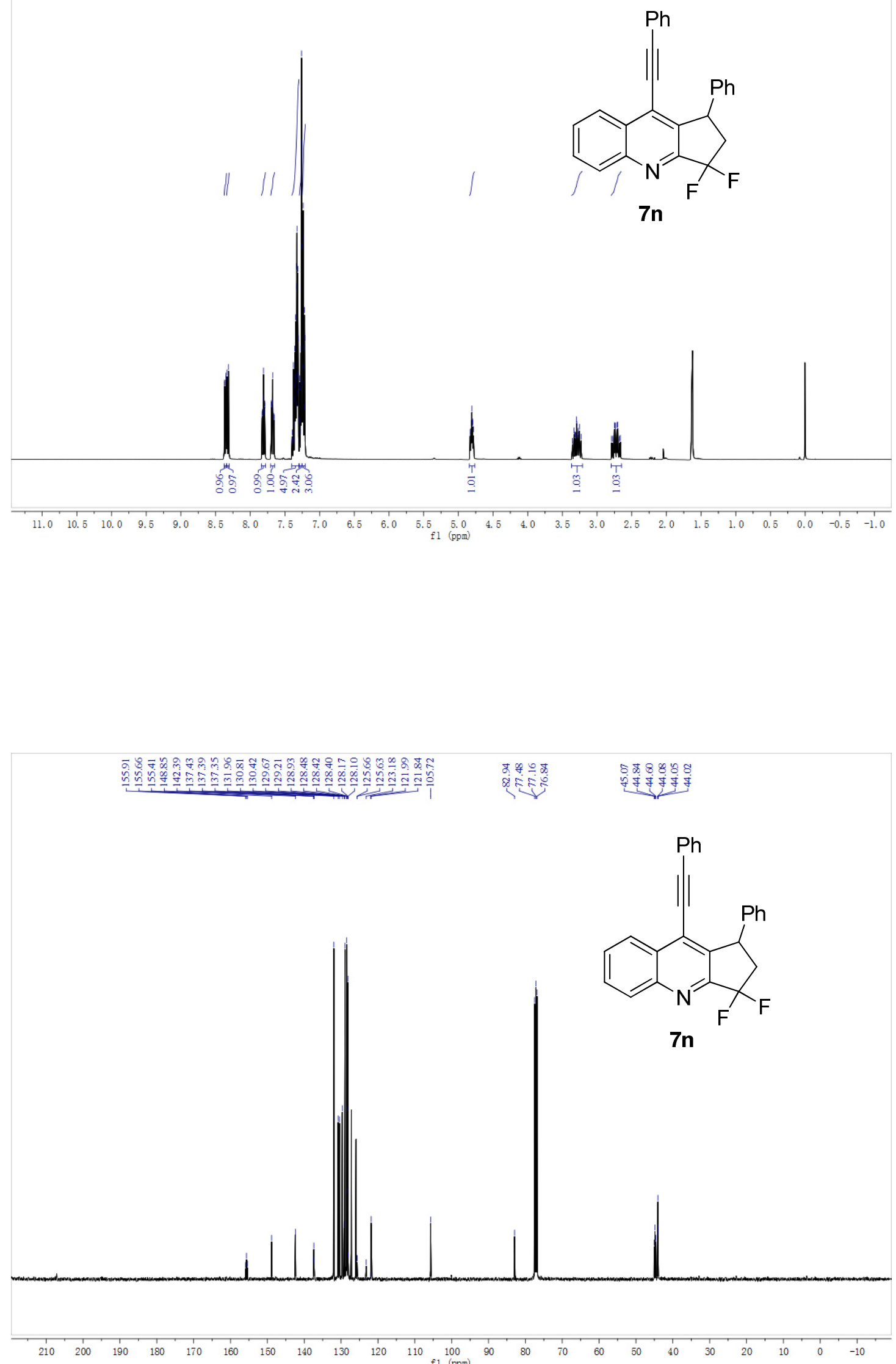


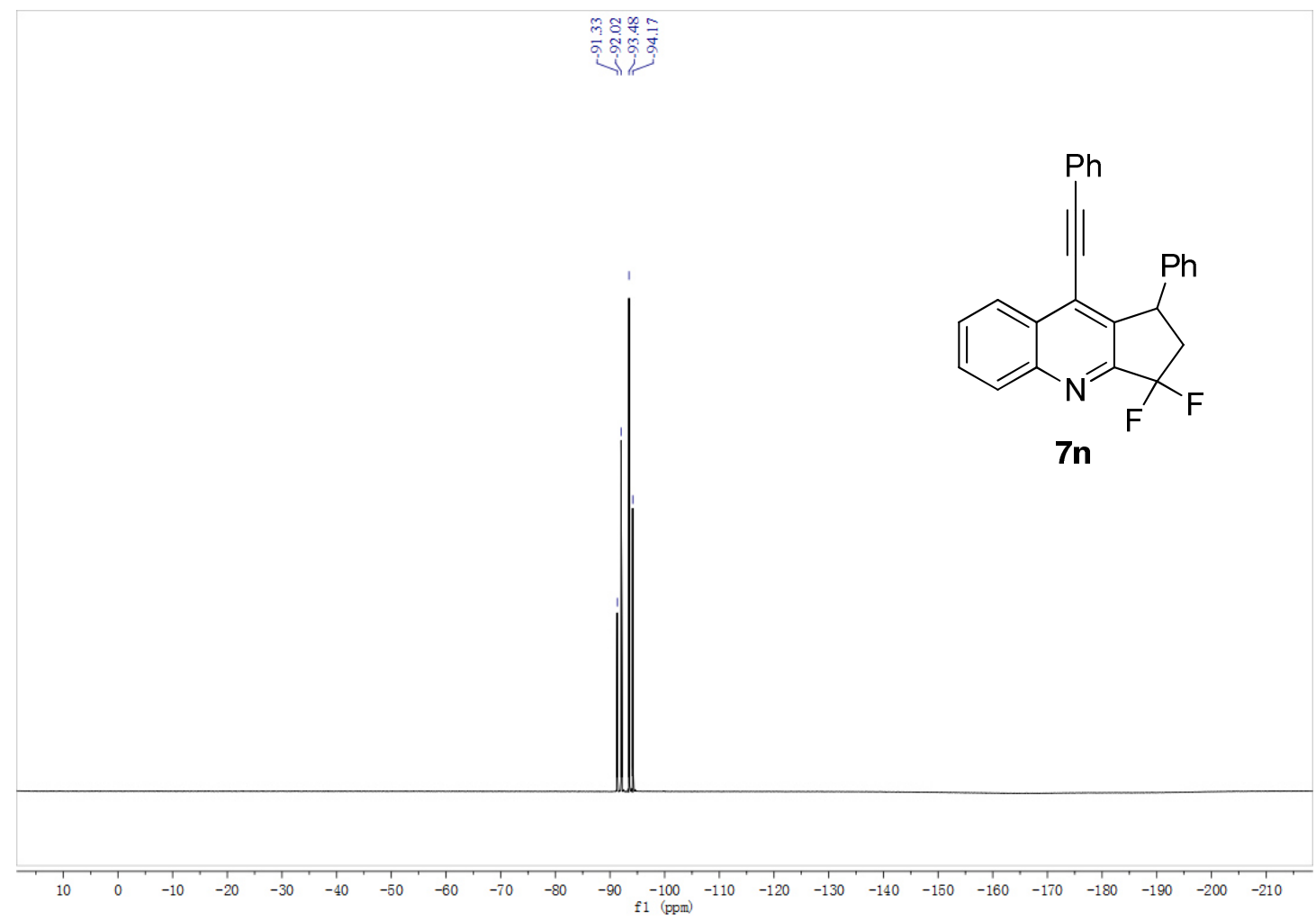

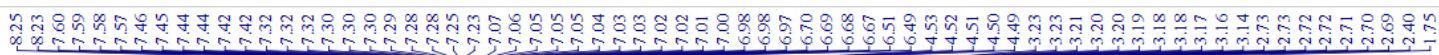

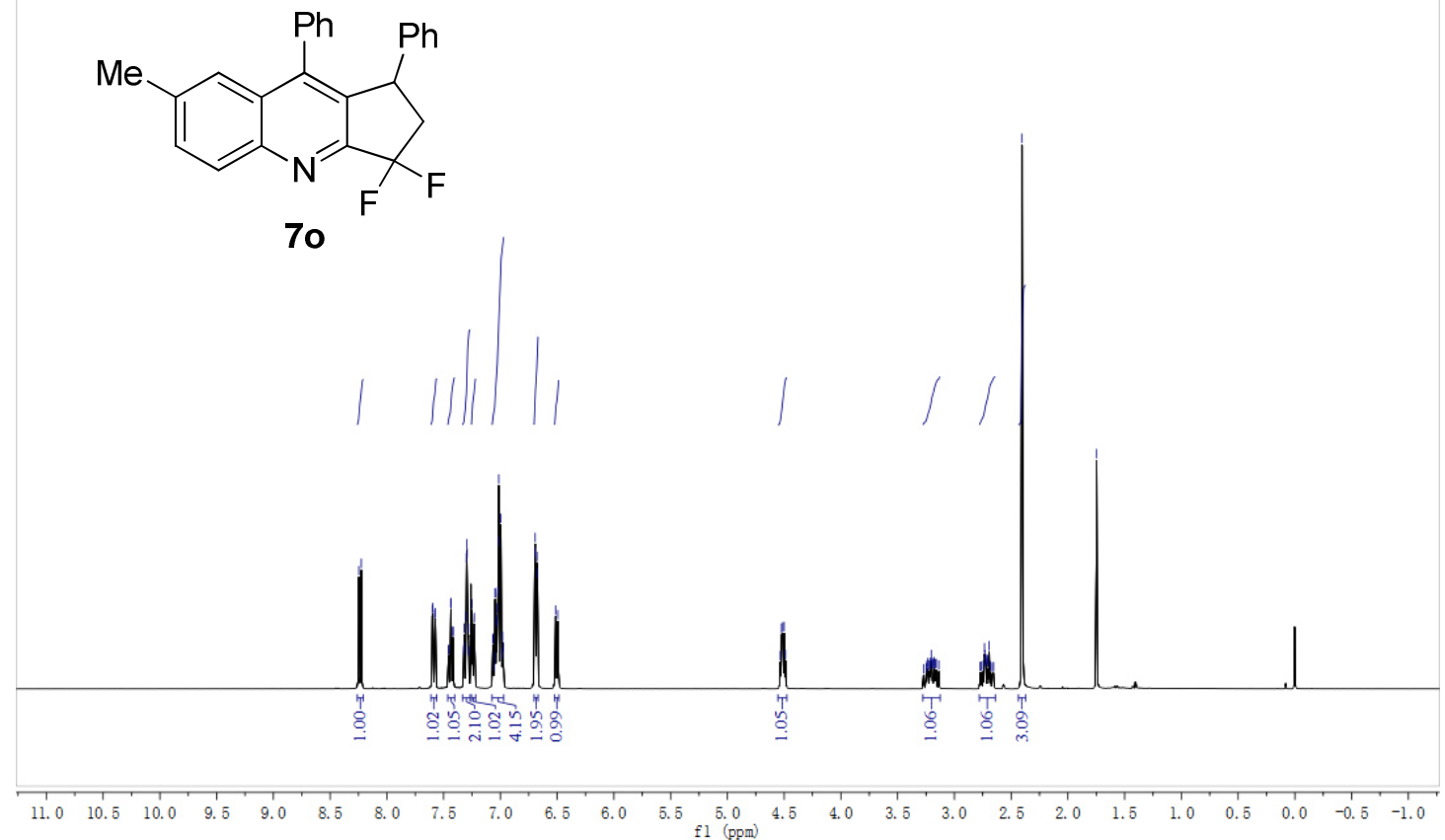



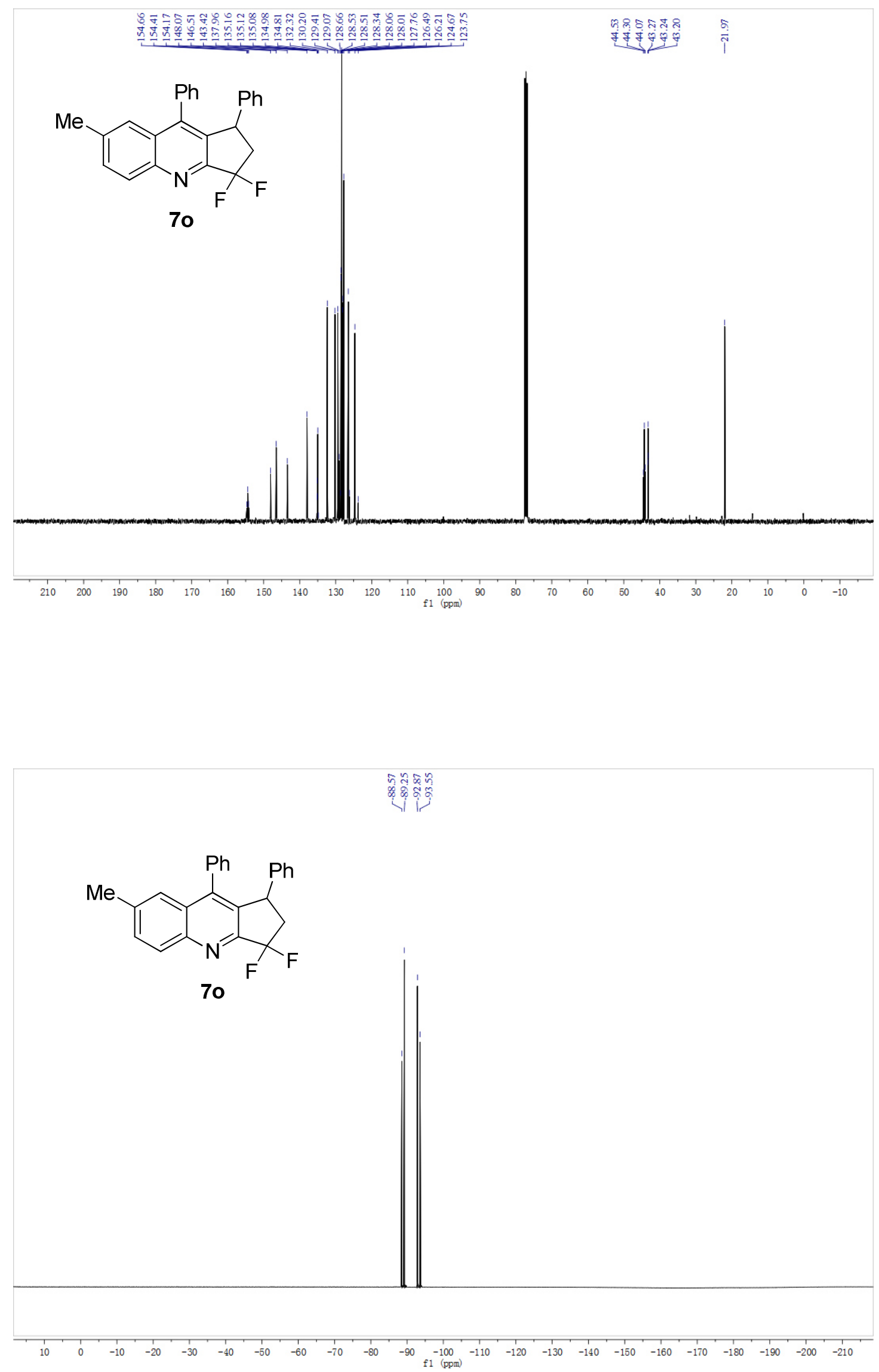

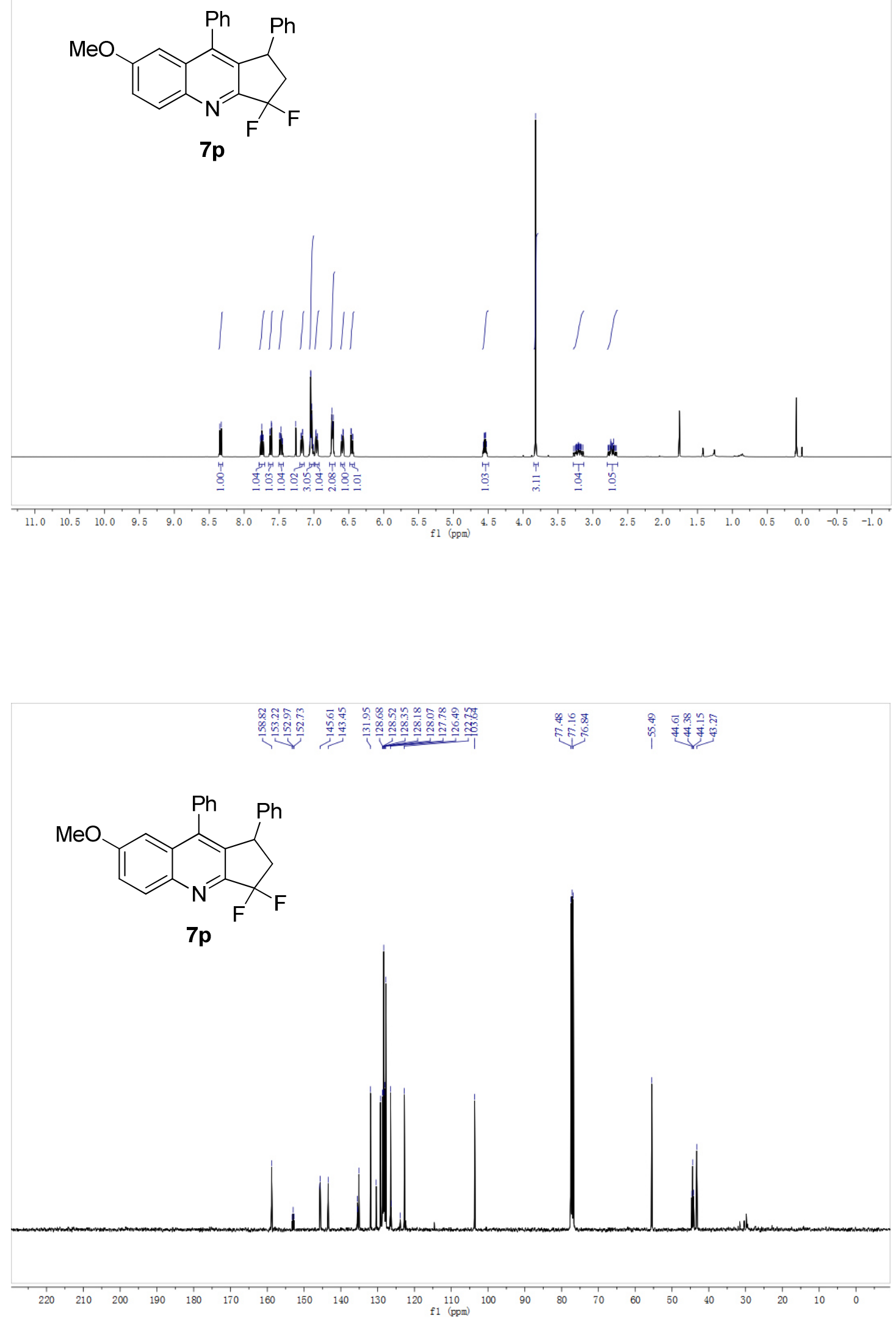


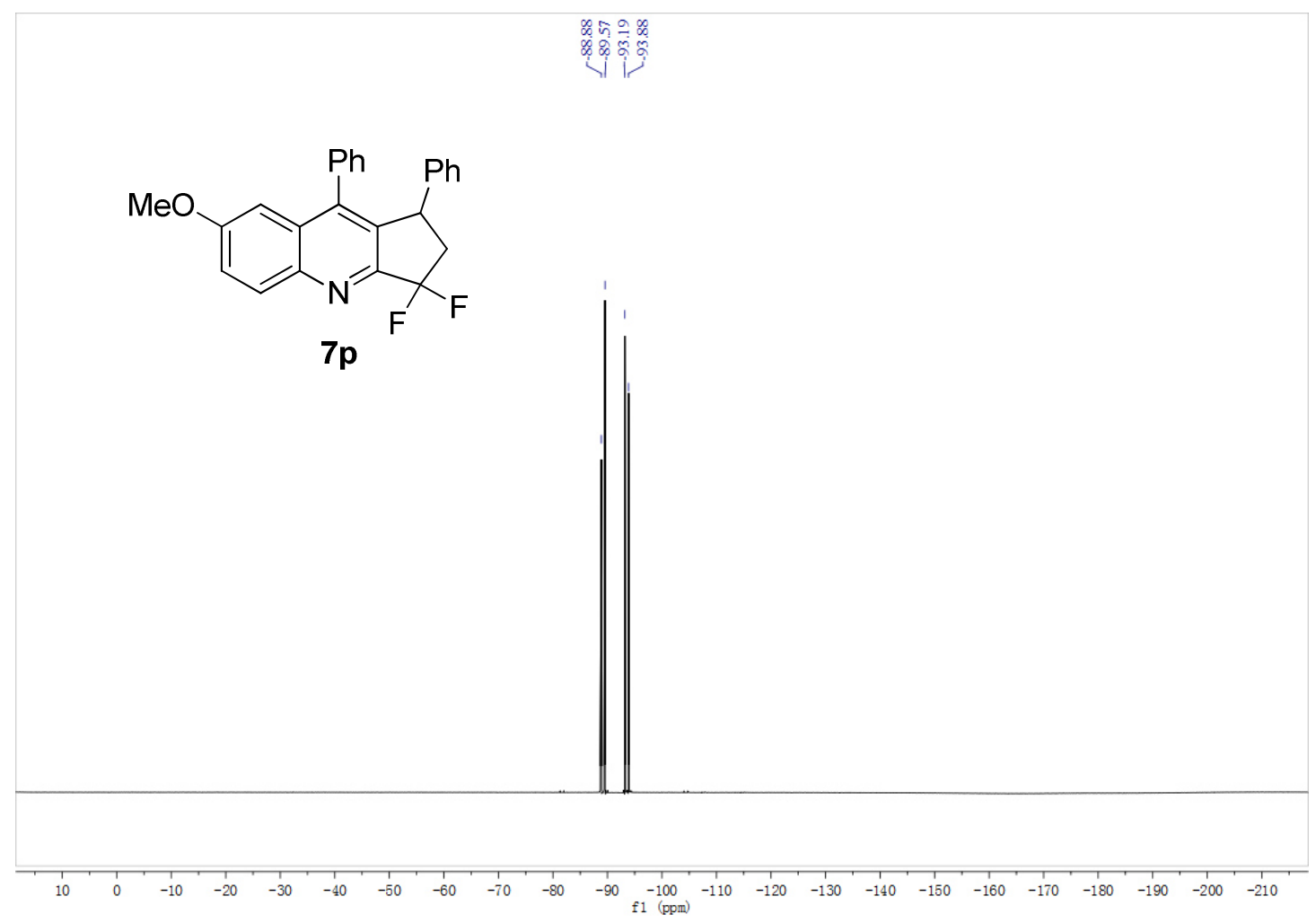

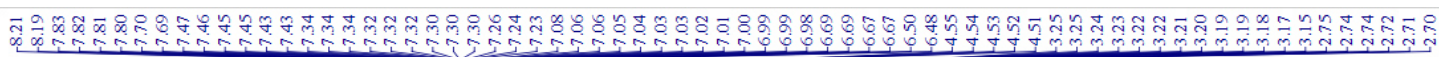

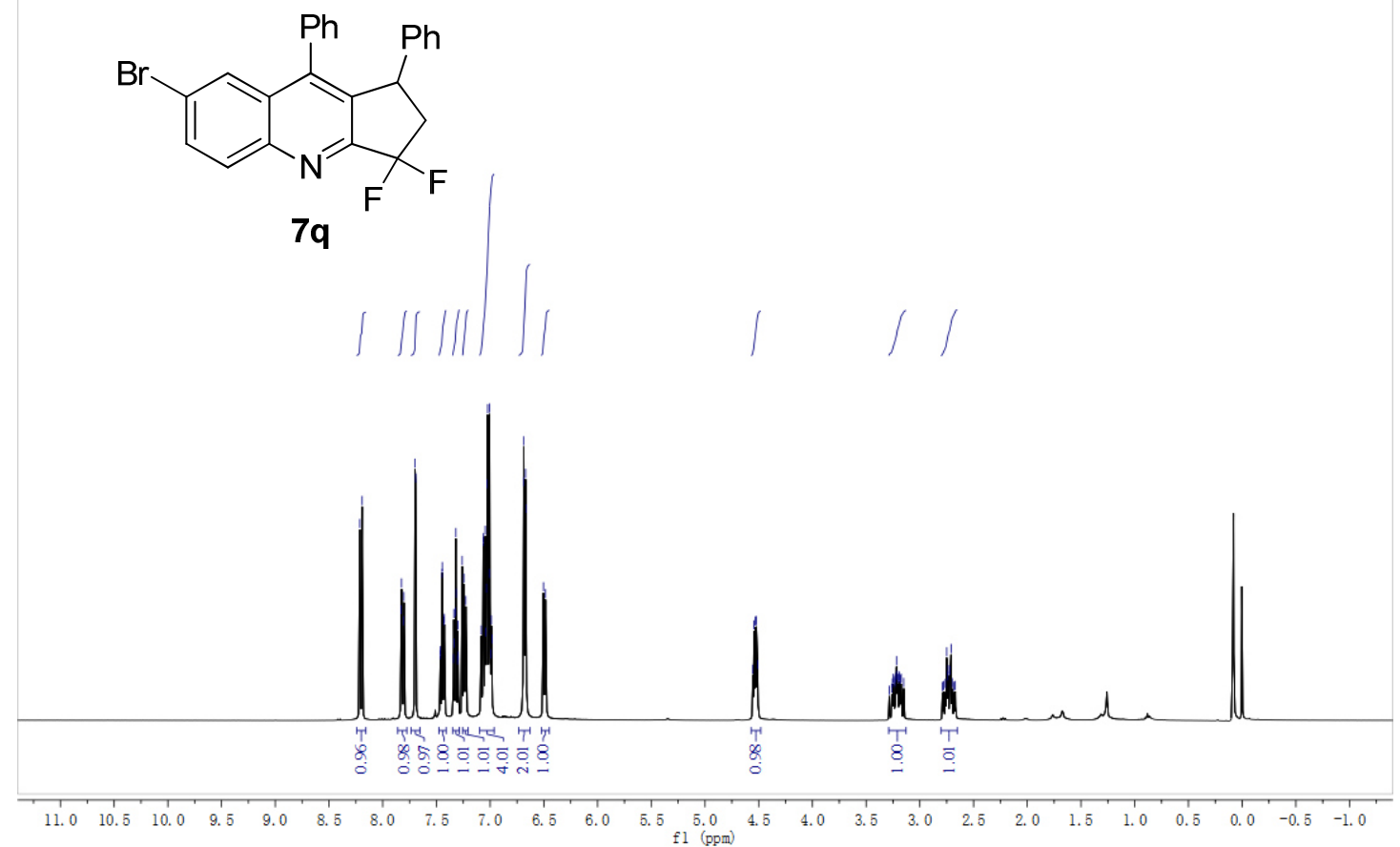



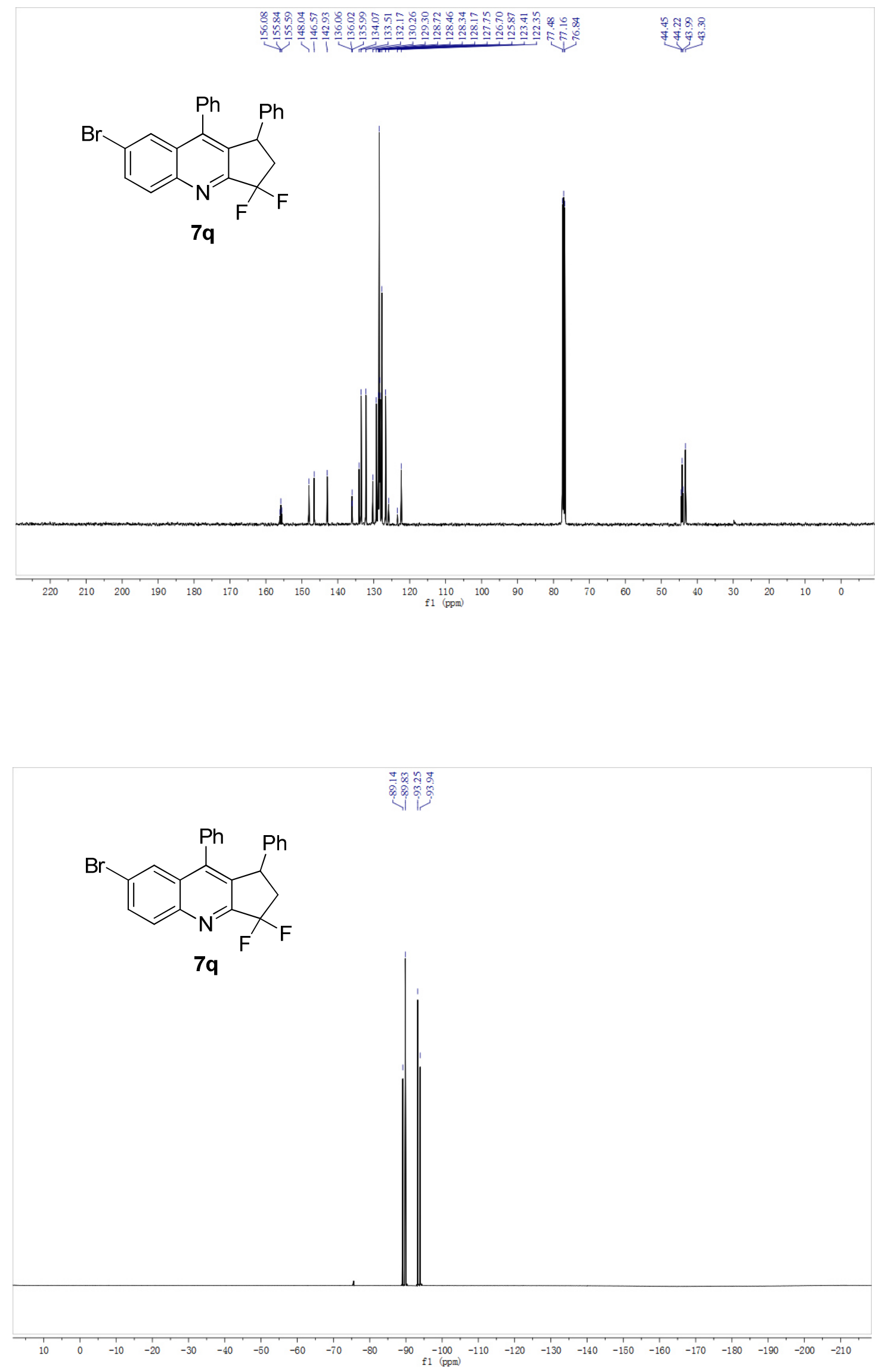
<smiles>Cc1cccc2c(-c3ccccc3)c3c(nc12)C(F)(F)CC3c1ccccc1</smiles>

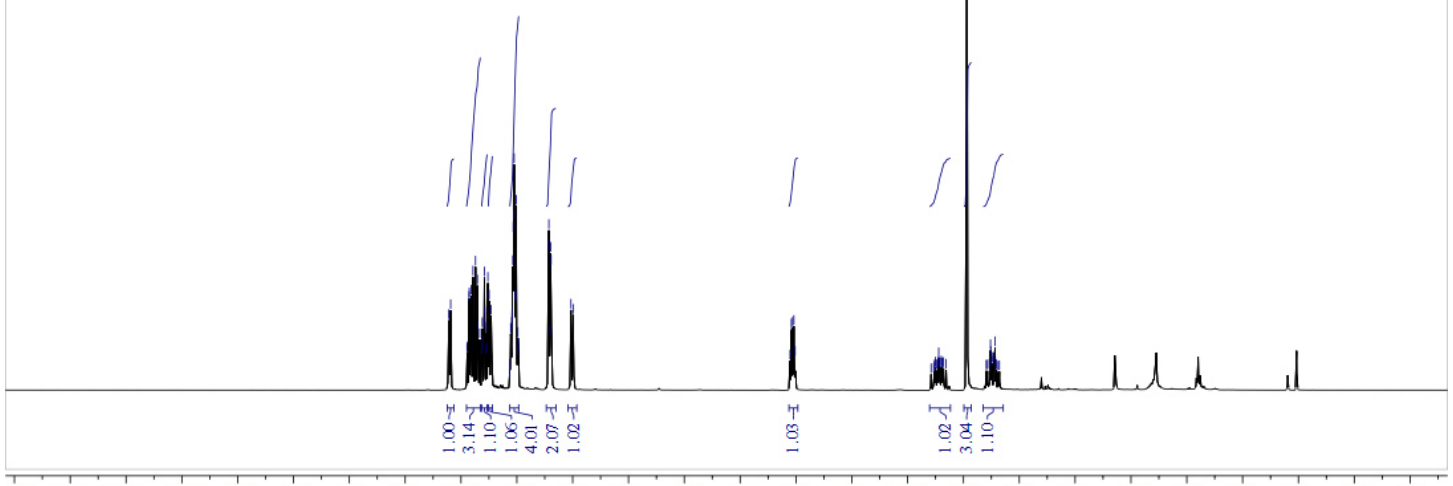

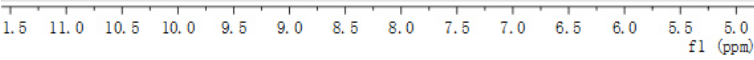

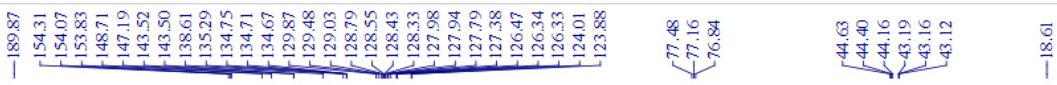<smiles>Cc1cccc2c(-c3ccccc3)c3c(nc12)C(F)(F)CC3c1ccccc1</smiles>

$\begin{array}{lllll}210 & 200 & 190 & 180 & 170\end{array}$

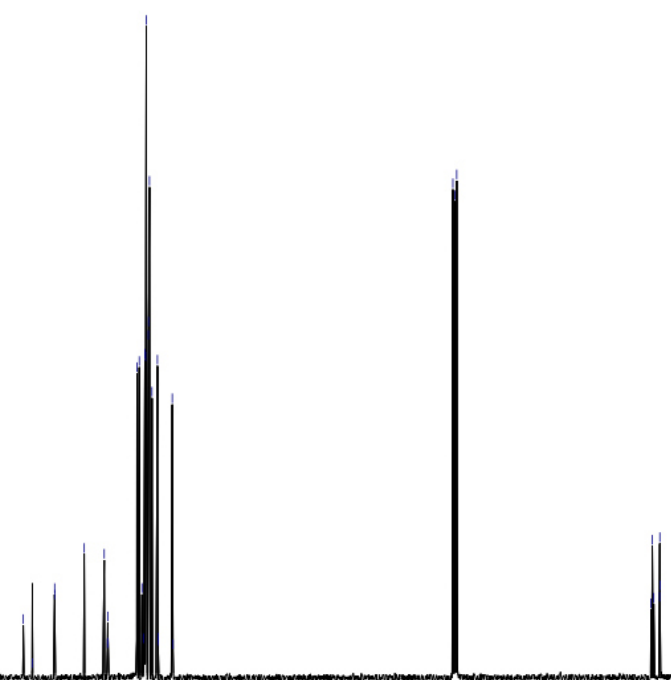




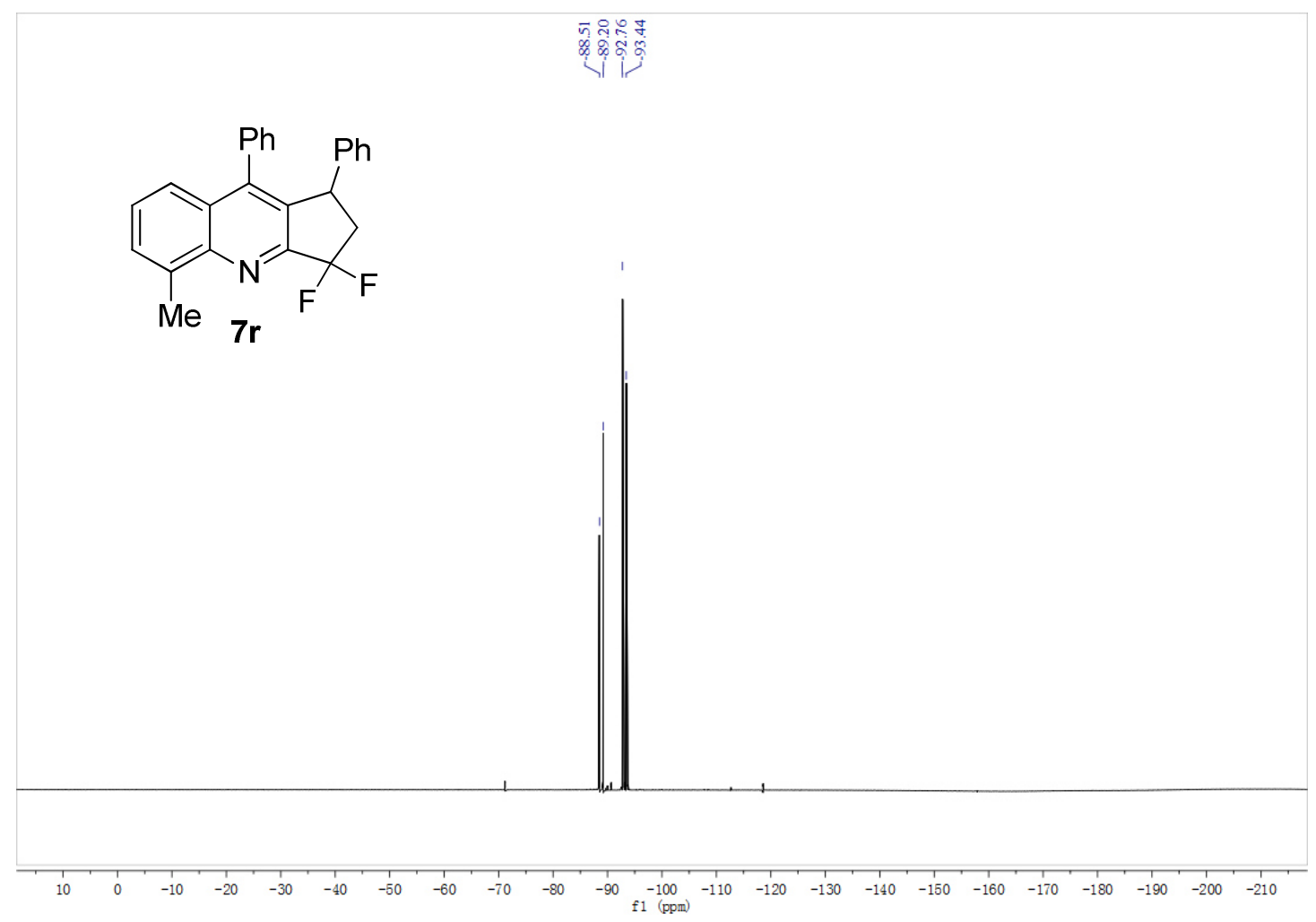

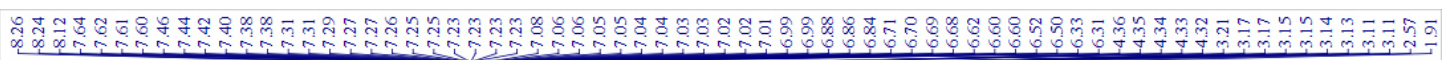<smiles>Cc1ccc2c(-c3ccccc3)c3c(nc2c1)C(F)(F)CC3c1ccccc1</smiles>

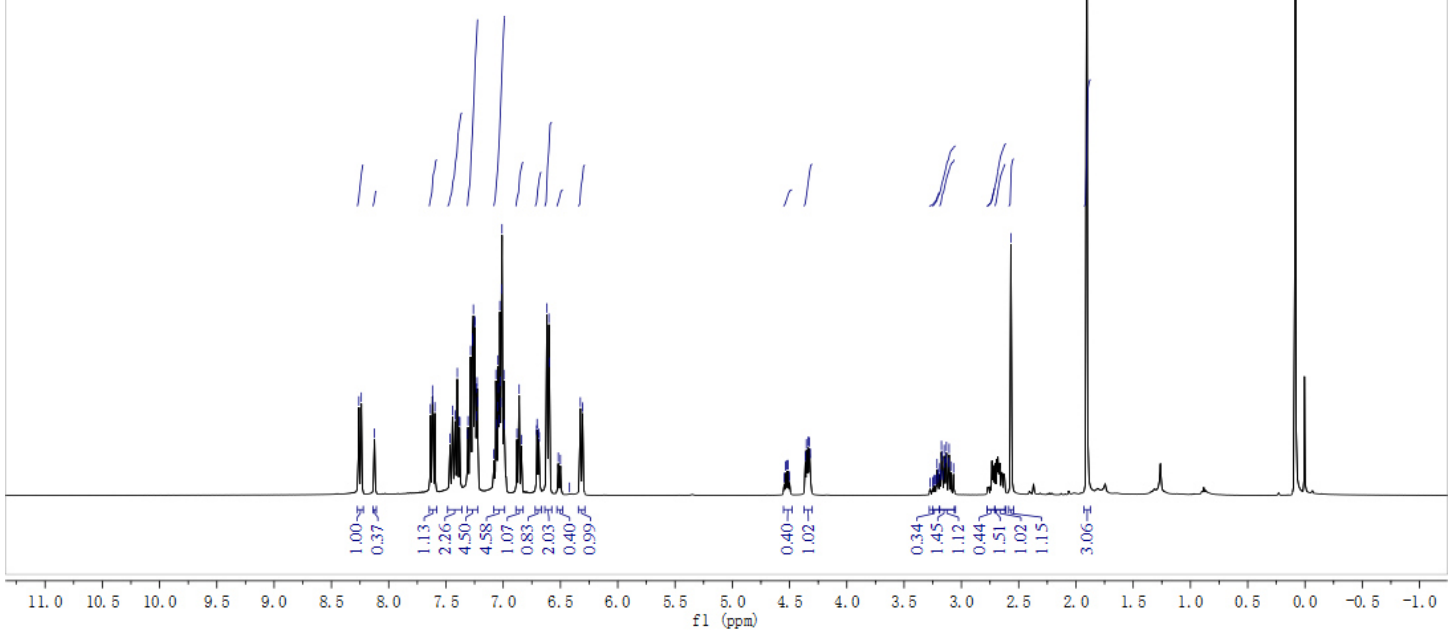




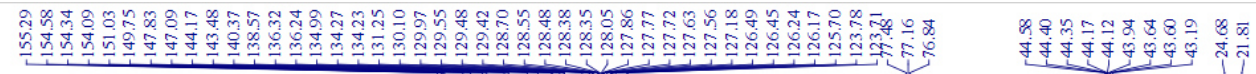<smiles>Cc1ccc2c(-c3ccccc3)c3c(nc2c1)C(F)(F)CC3c1ccccc1</smiles>

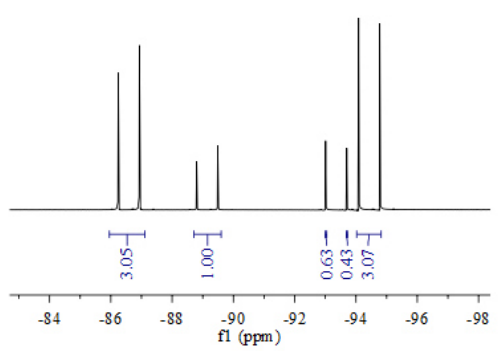<smiles>Cc1ccc2c(-c3ccccc3)c3c(nc2c1)C(F)(F)CC3c1ccccc1</smiles> 


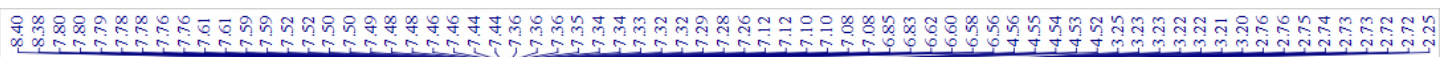<smiles></smiles>
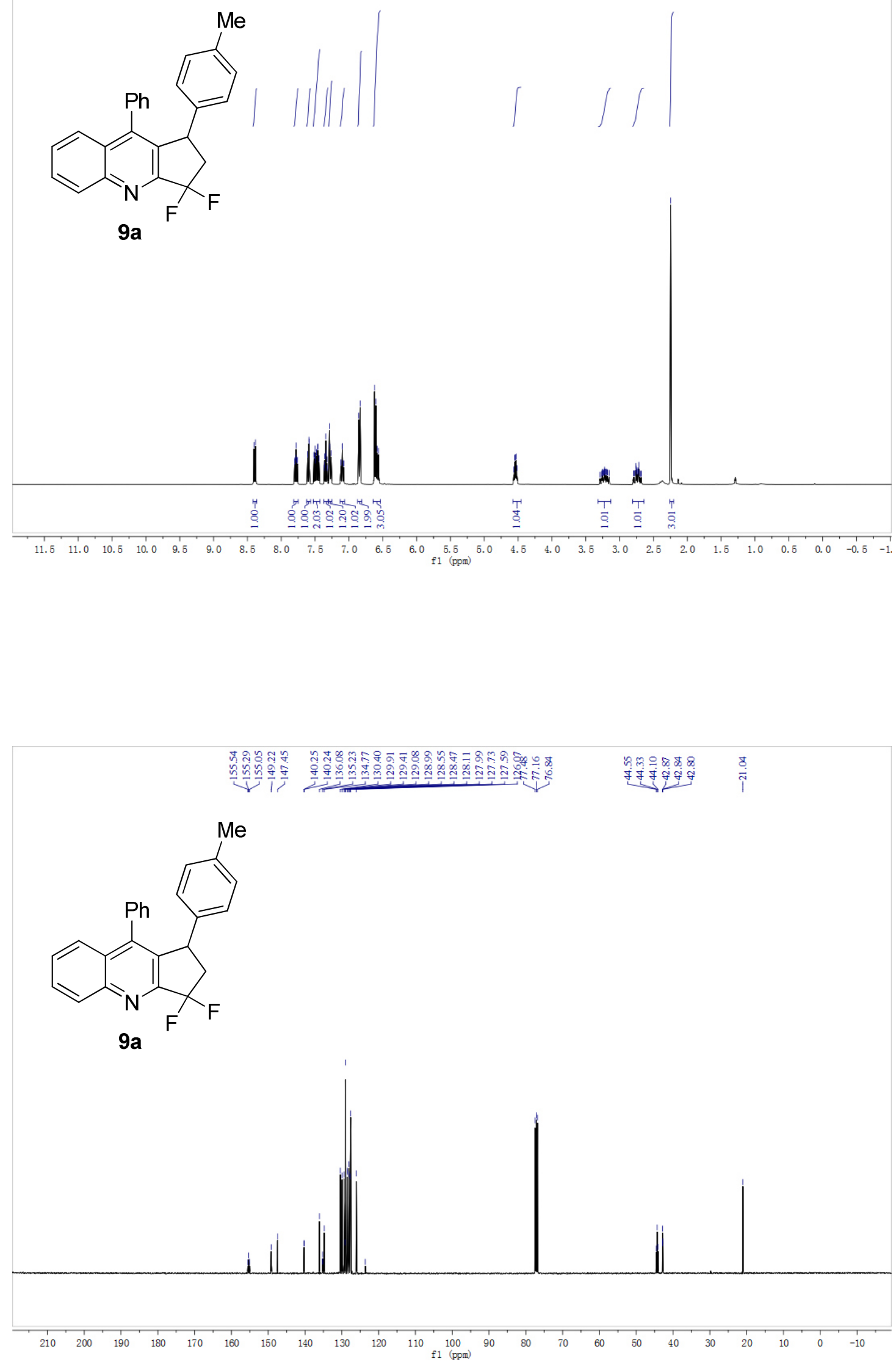


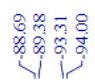<smiles>Cc1ccc(C2CC(F)(F)c3nc4ccccc4c(-c4ccccc4)c32)cc1</smiles>

$\mid$

\begin{tabular}{rrrrrrrrrrrrrrrrrrrrrrrrrrrr}
\hline 10 & 0 & -10 & -20 & -30 & -40 & -50 & -60 & -70 & -80 & -90 & -100 & -110 & -120 & -130 & -140 & -150 & -160 & -170 & -180 & -190 & -200 & -210 \\
\hline
\end{tabular}

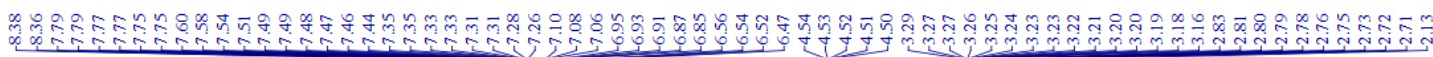<smiles>C#CC#CC1CC(F)(F)c2nc3ccccc3c(-c3ccccc3)c21</smiles>

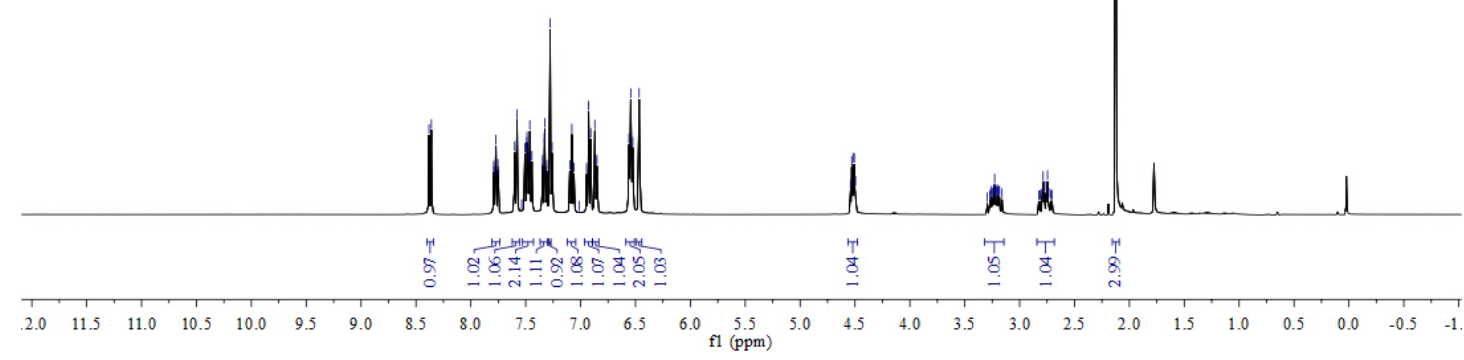



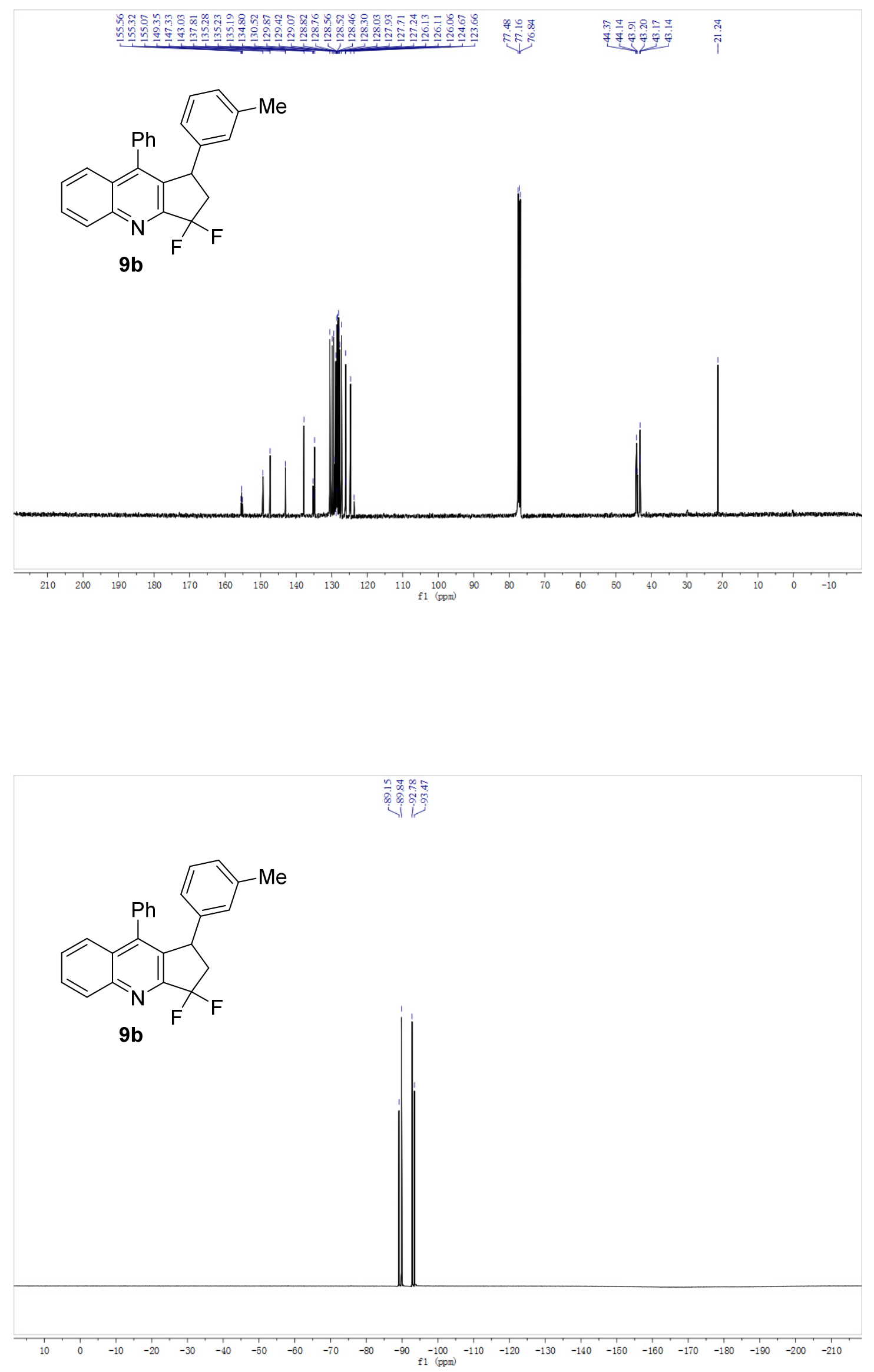

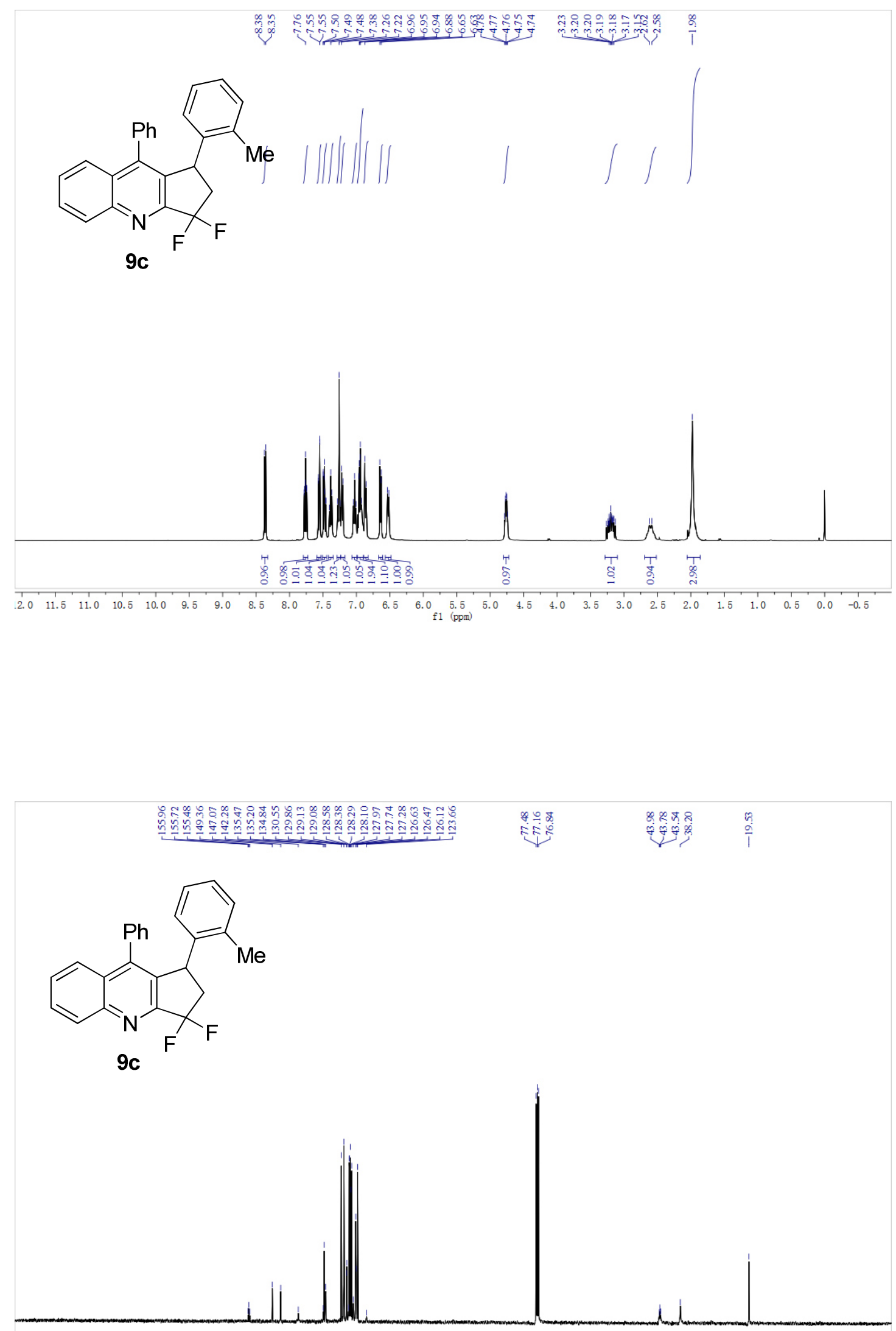

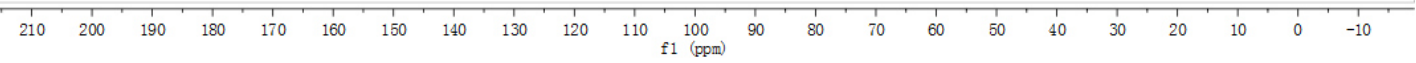


<smiles>Cc1ccccc1C1CC(F)(F)c2nc3ccccc3c(-c3ccccc3)c21</smiles>

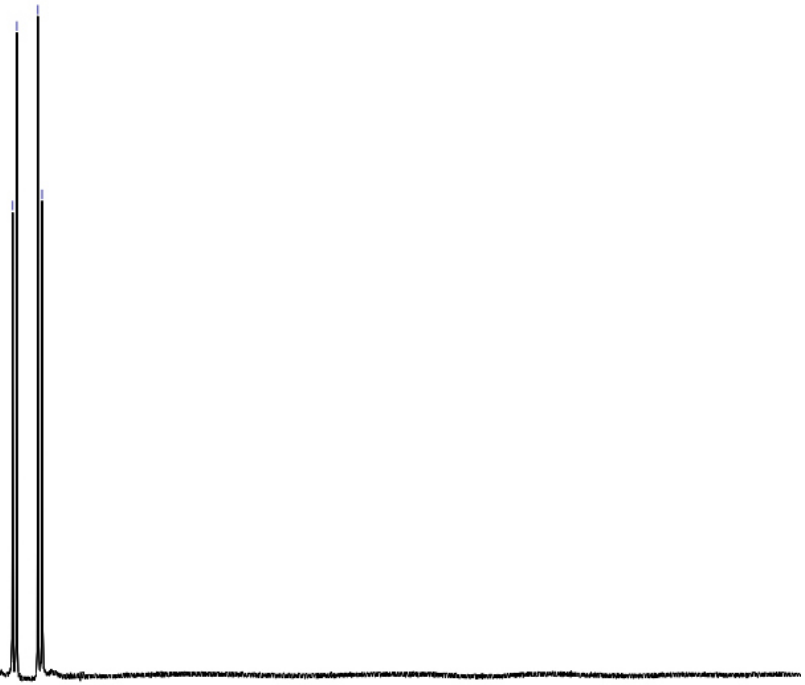

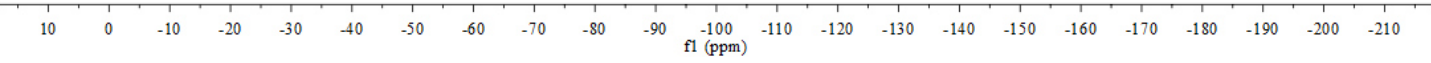

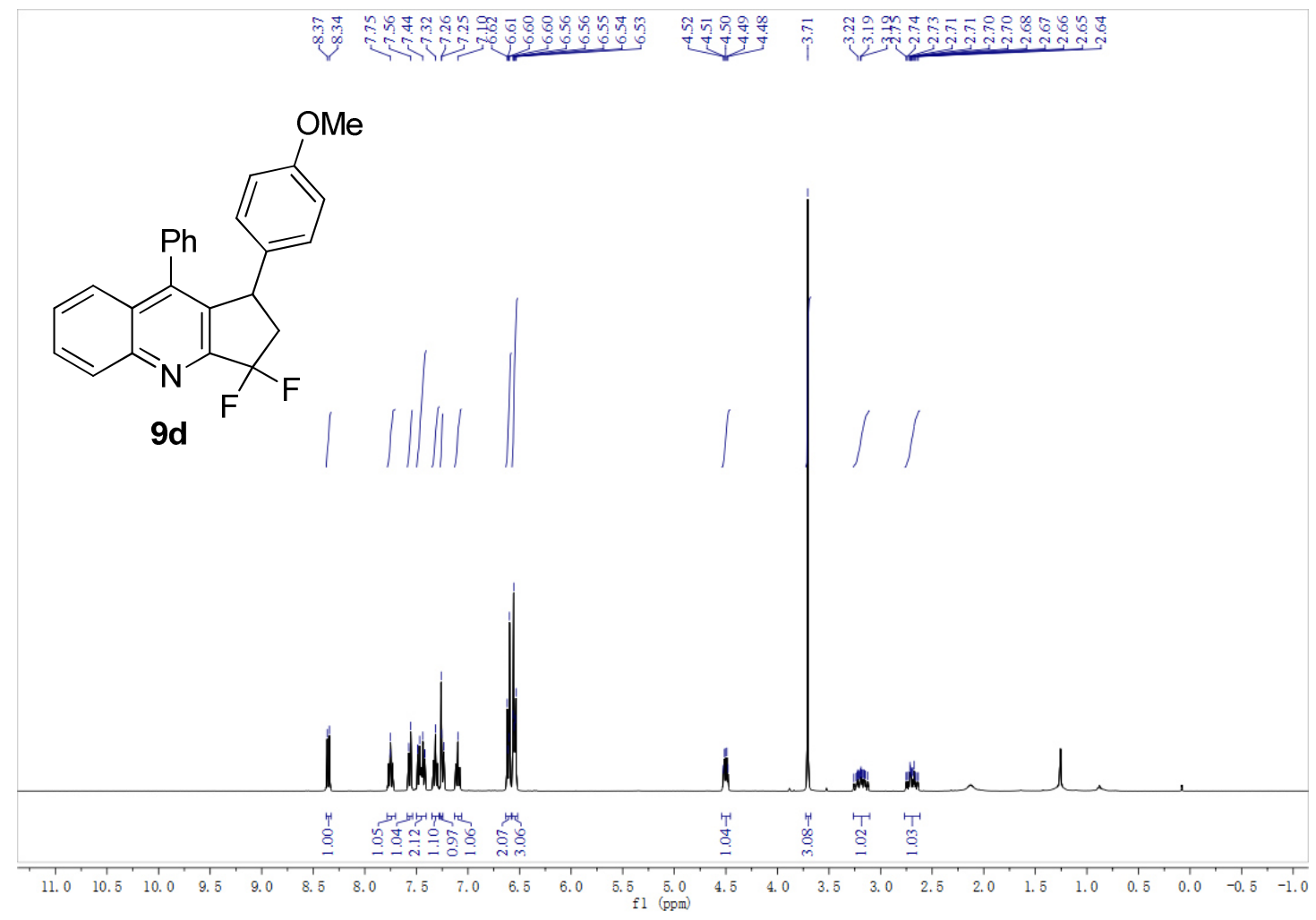



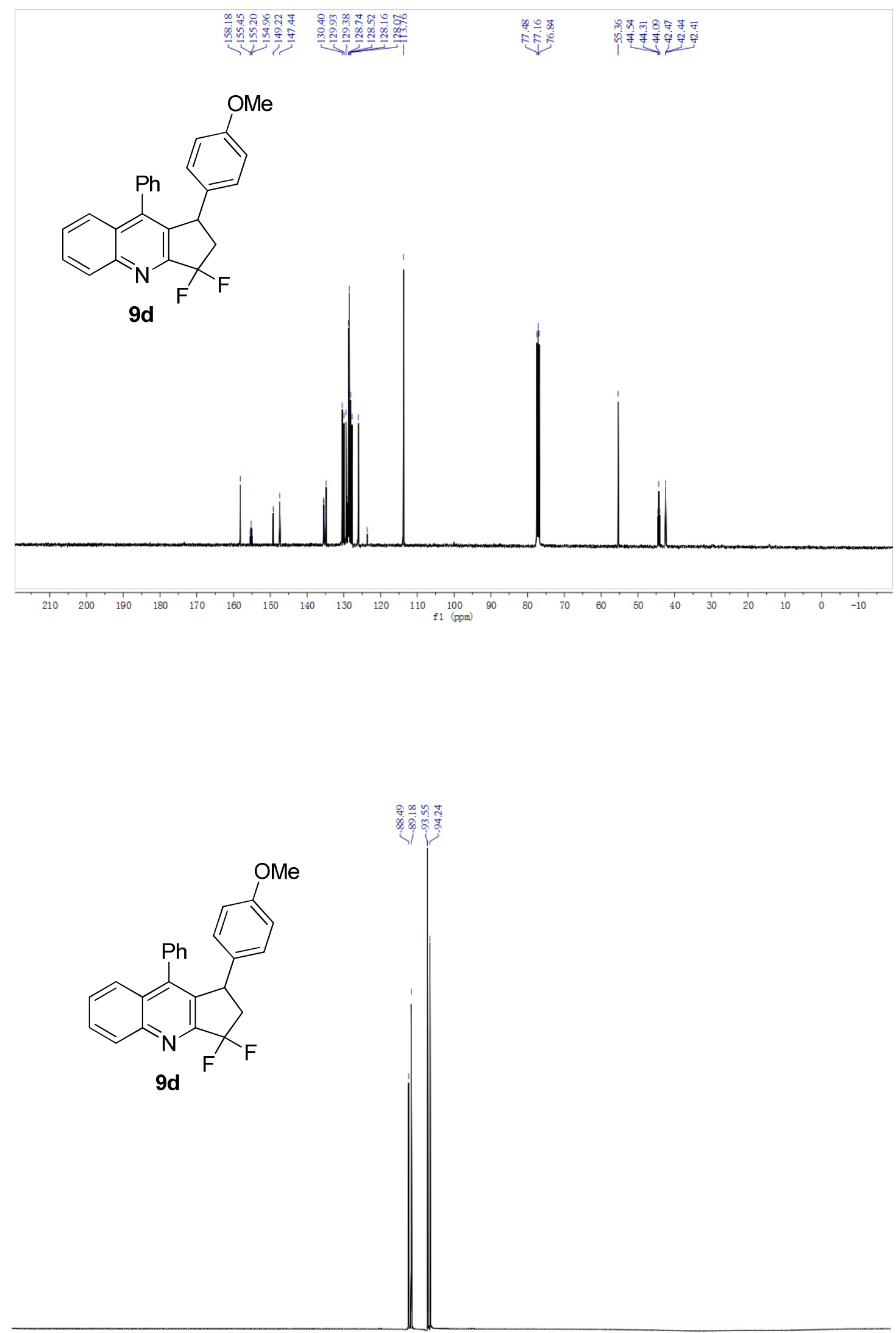

$\begin{array}{lllllllllllllllllllllll}10 & 0 & -10 & -20 & -30 & -40 & -50 & -60 & -70 & -80 & -90 & -100 & -110 & -120 & -130 & -140 & -150 & -160 & -170 & -180 & -190 & -200 & -210\end{array}$ 

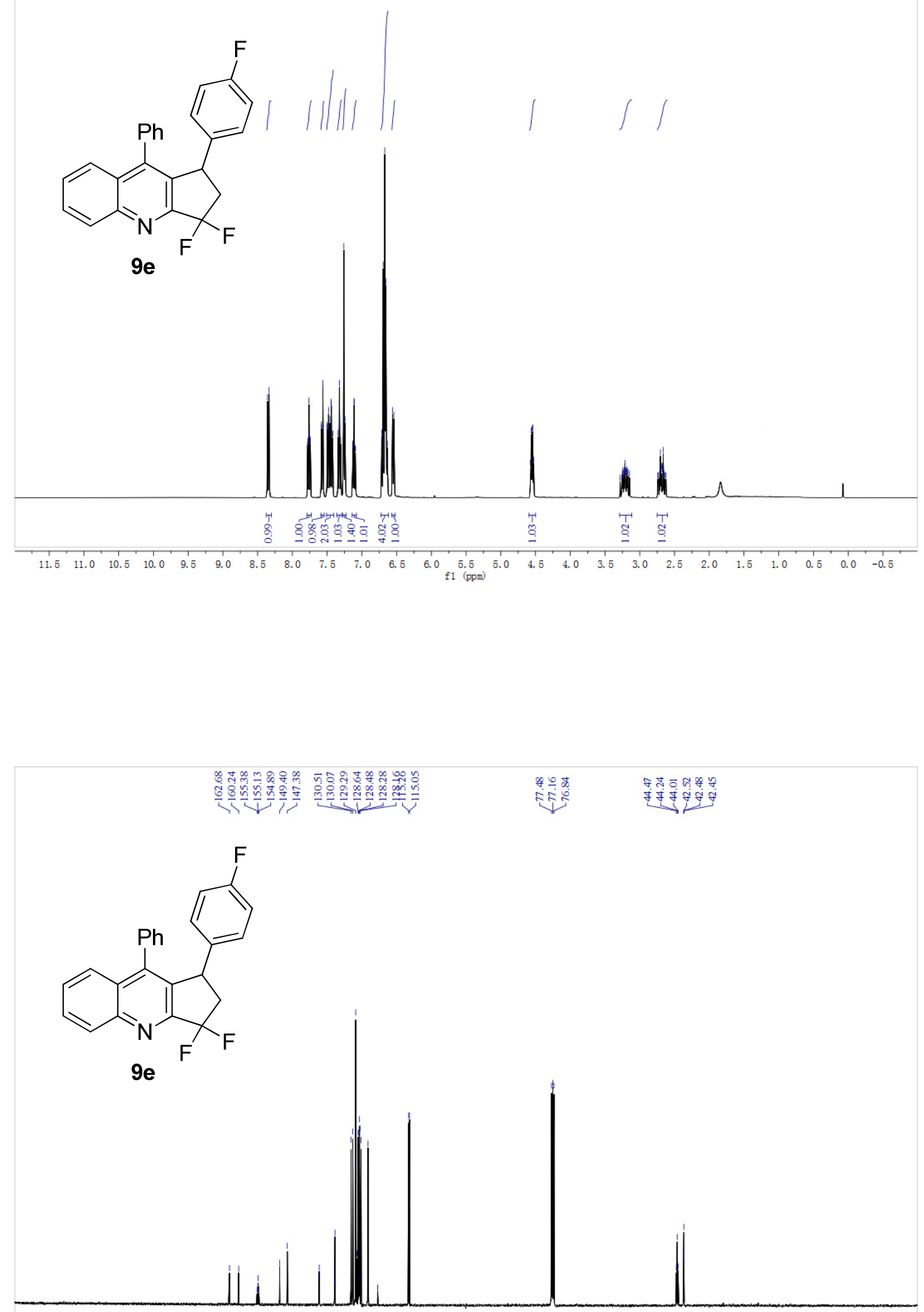

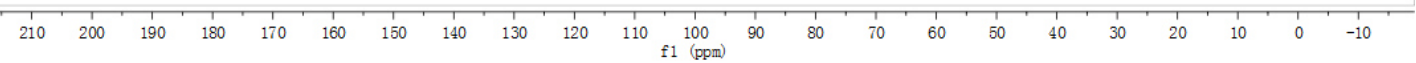




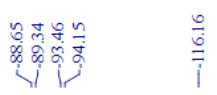<smiles>Fc1ccc(C2CC(F)(F)c3nc4ccccc4c(-c4ccccc4)c32)cc1</smiles>

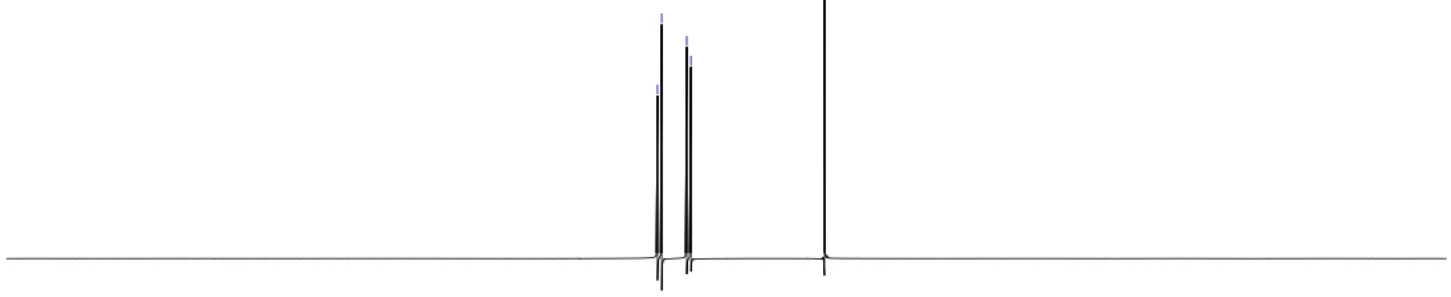

\begin{tabular}{rllllllllllllllllllllllll}
\hline 10 & 0 & -10 & -20 & -30 & -40 & -50 & -60 & -70 & -80 & -90 & -100 & -110 & -120 & -130 & -140 & -150 & -160 & -170 & -180 & -190 & -200 & -210
\end{tabular}

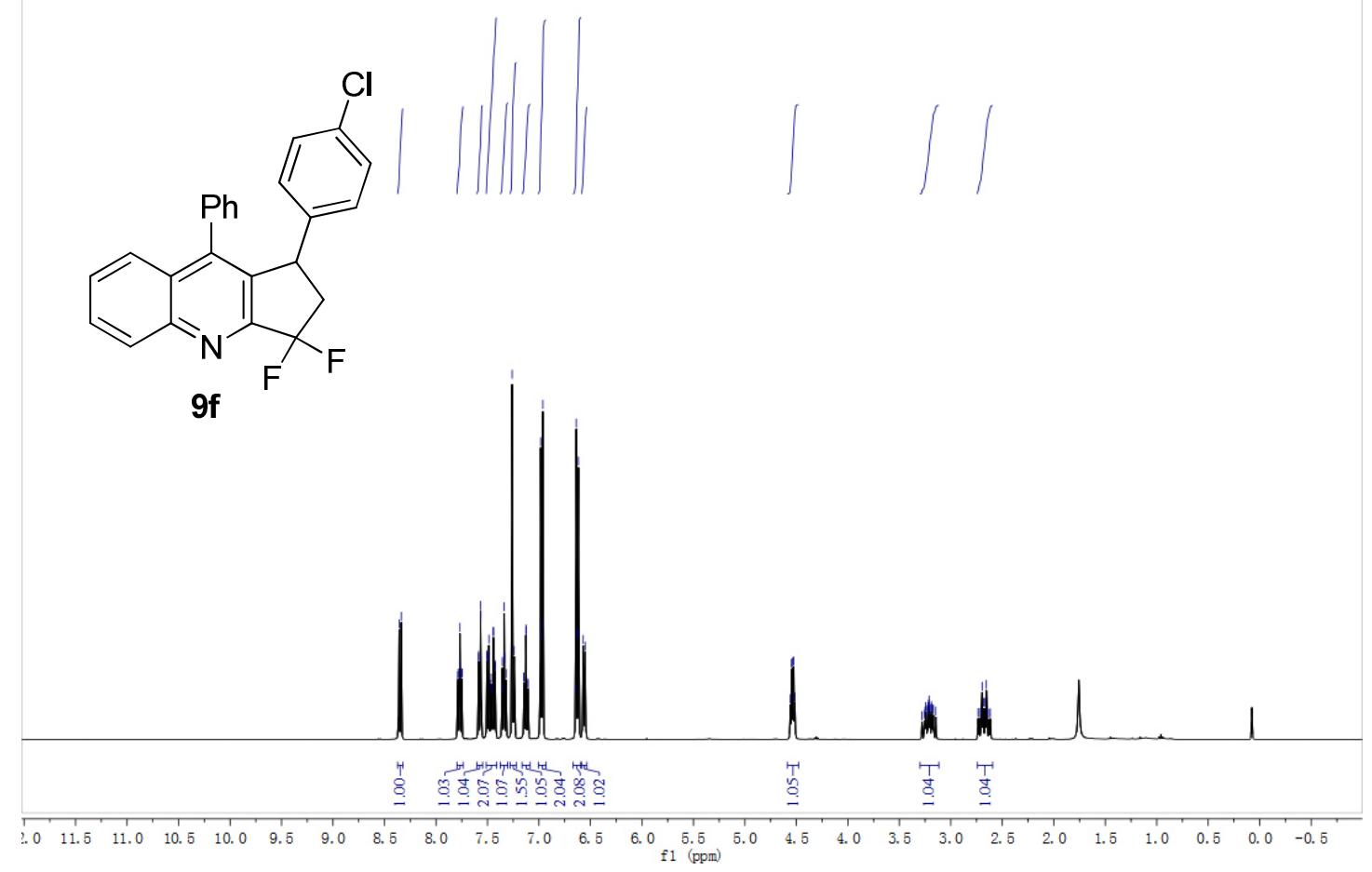



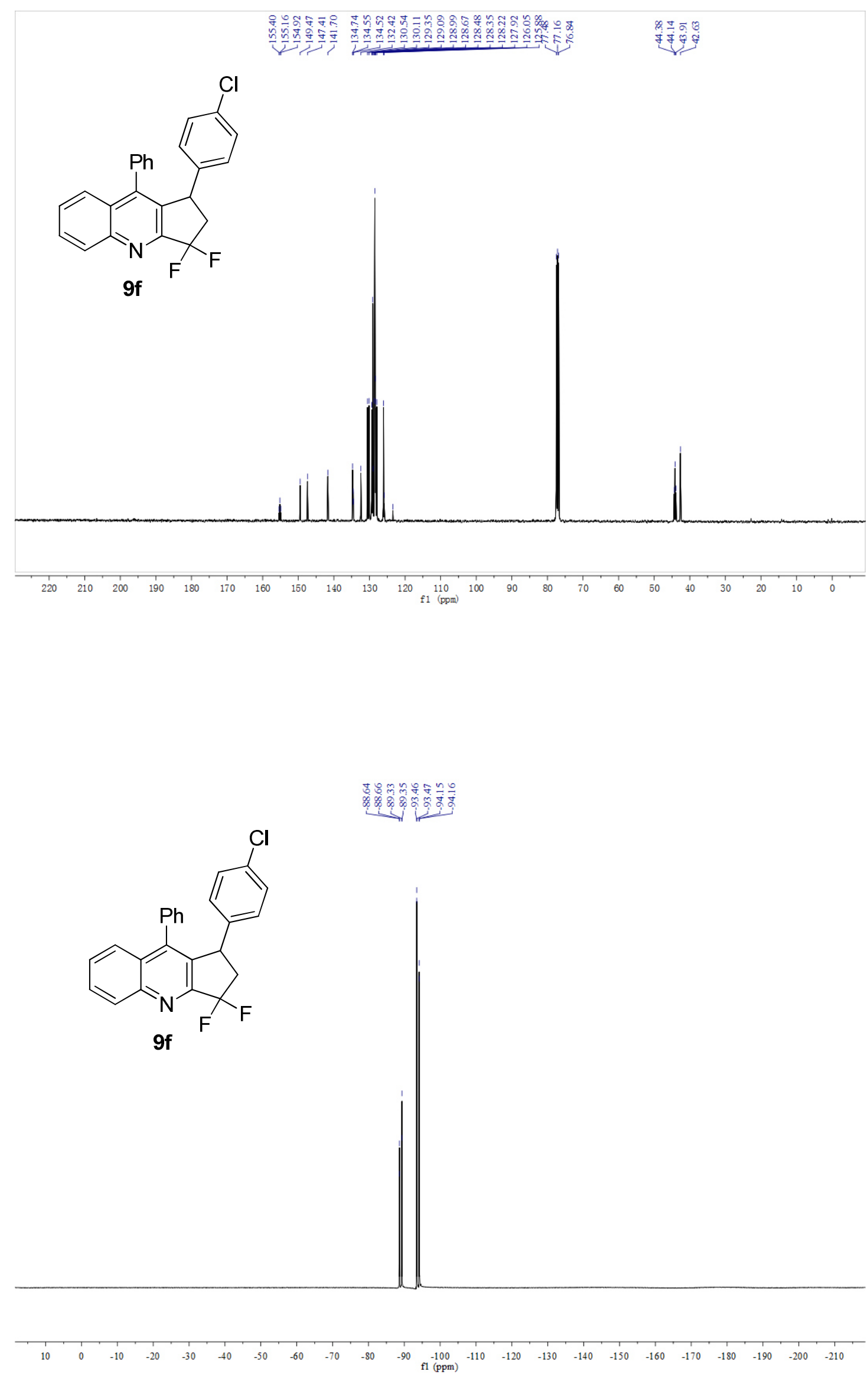

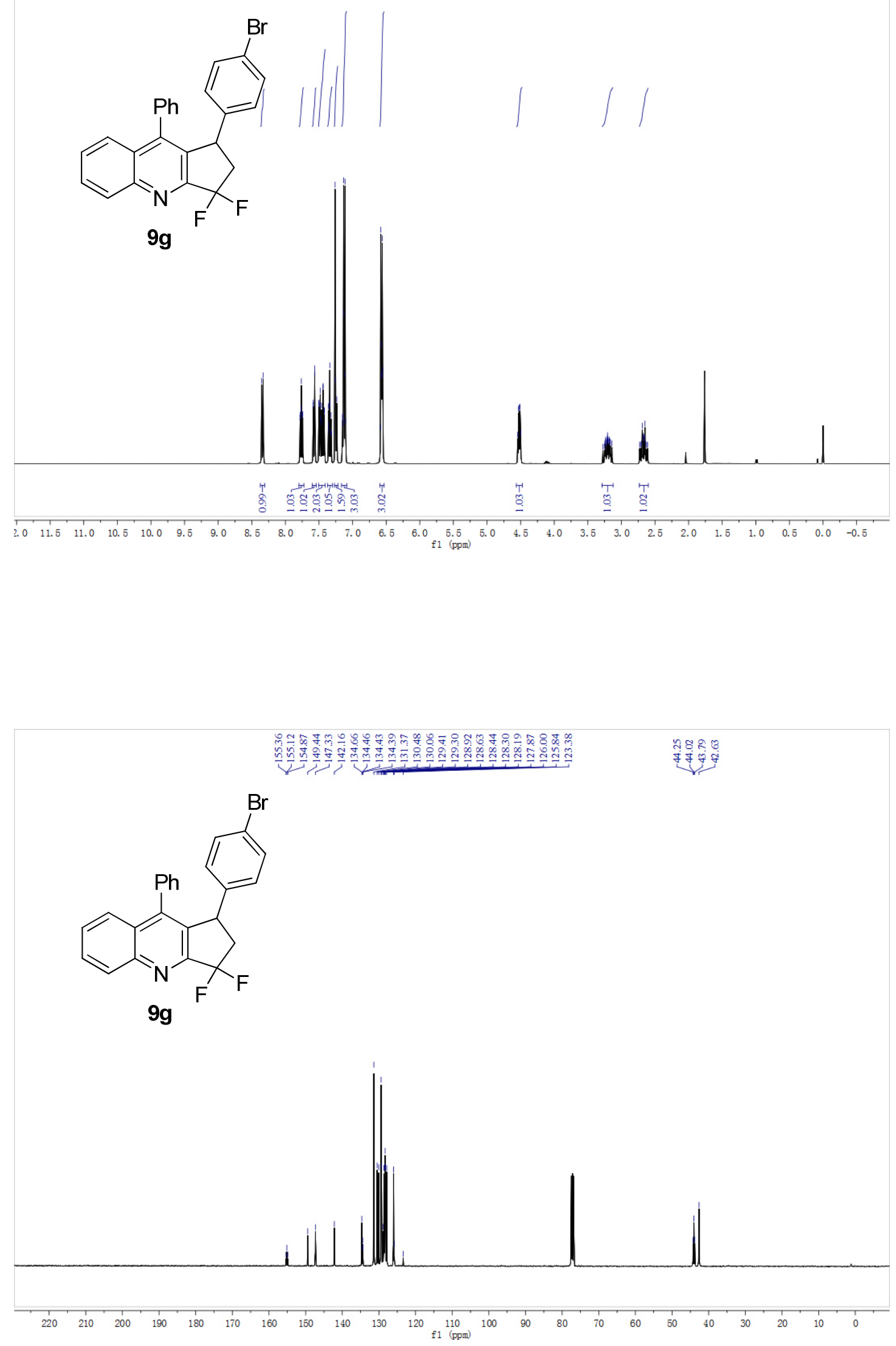


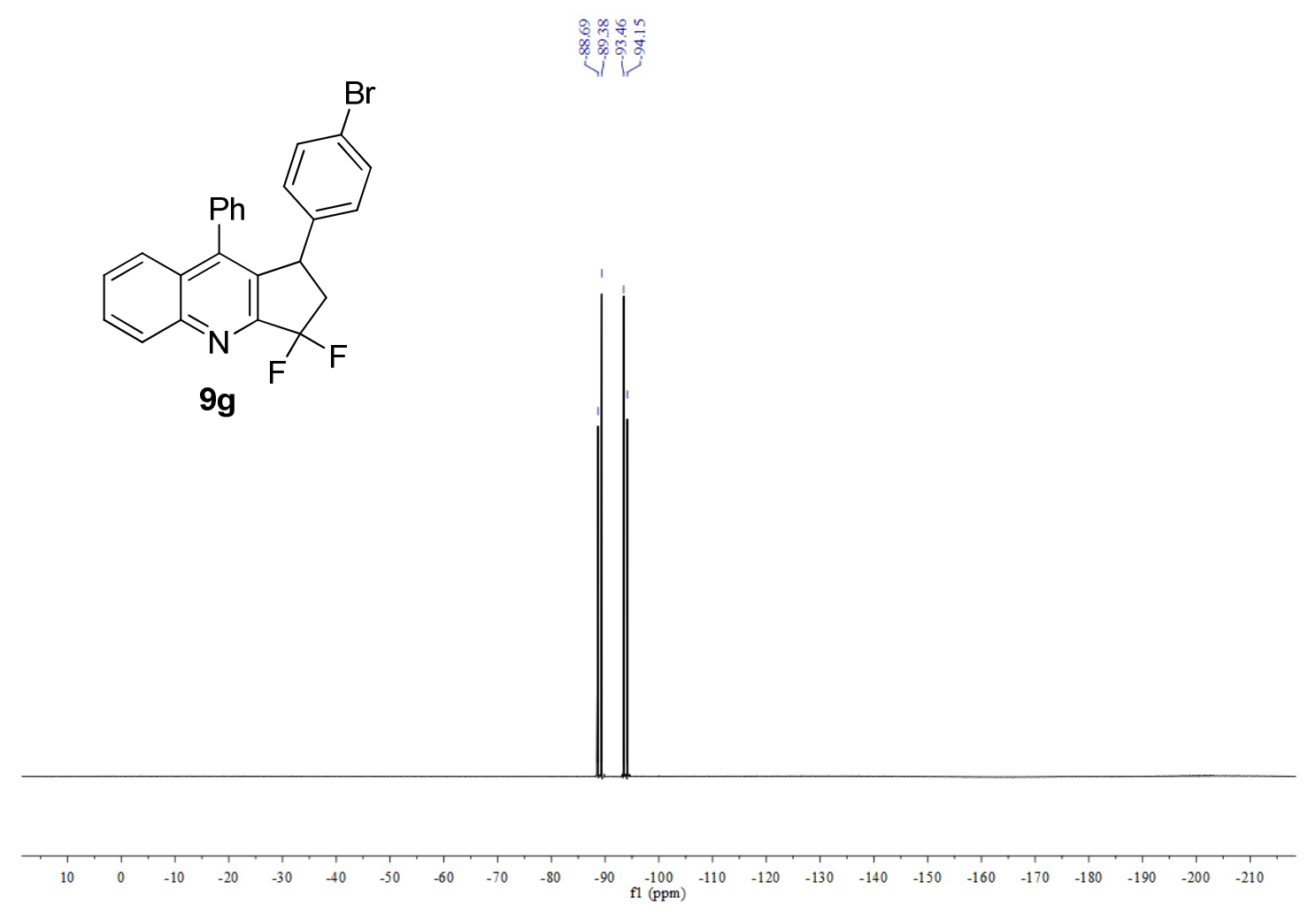

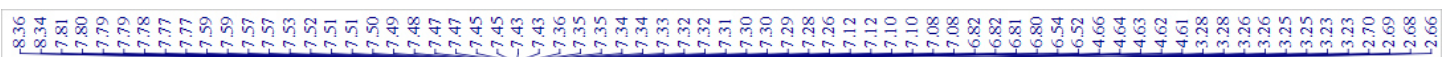

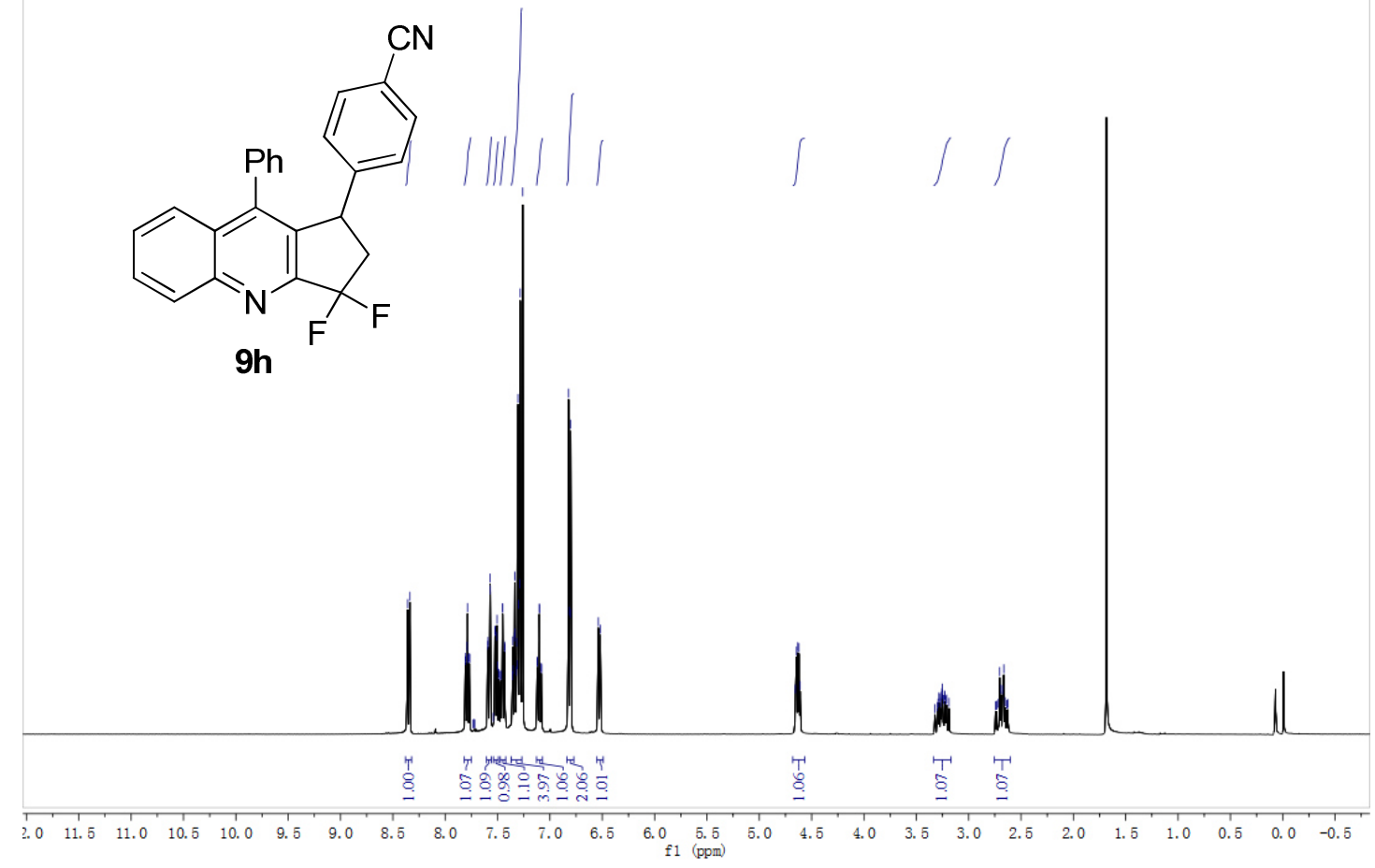



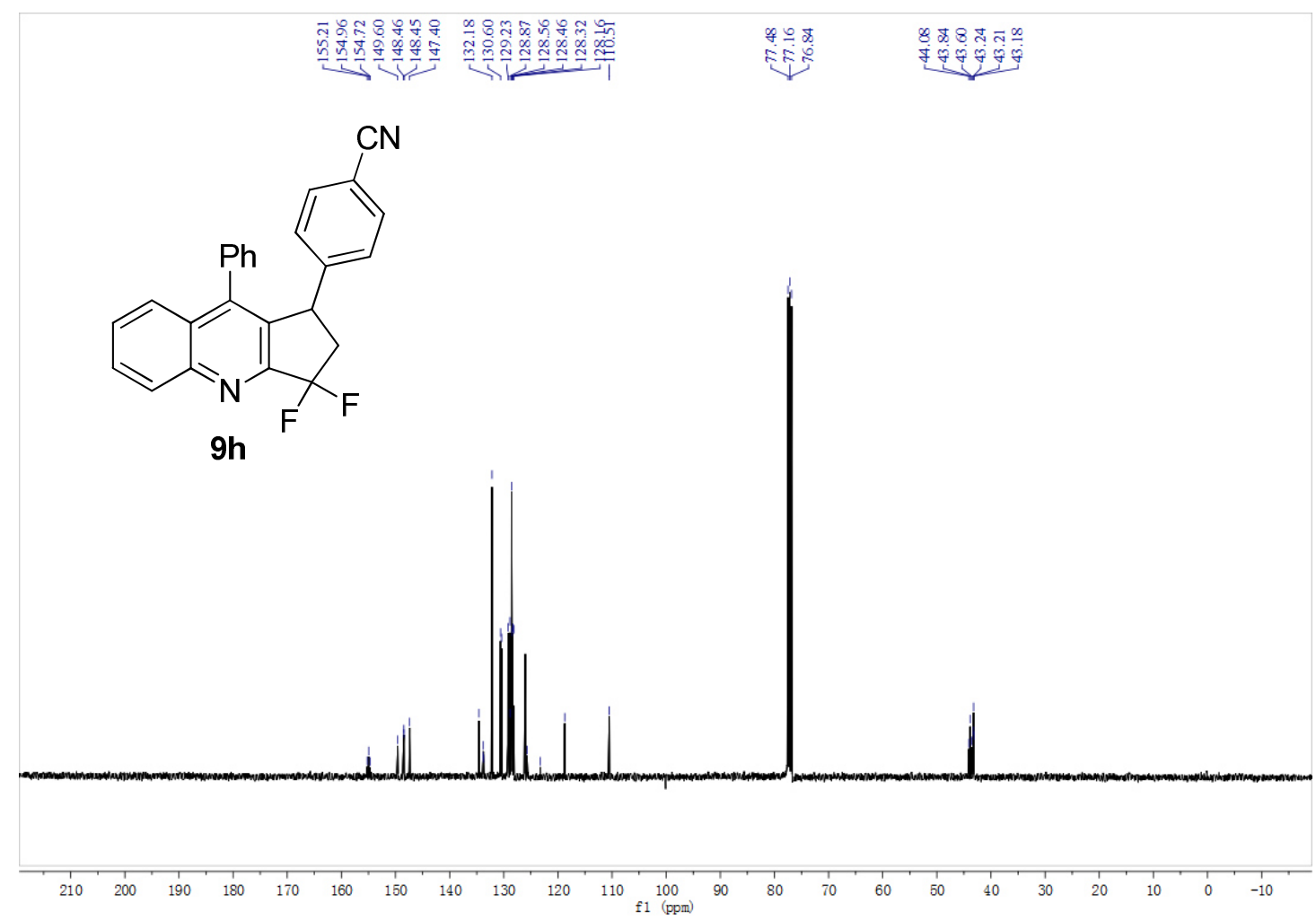

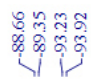

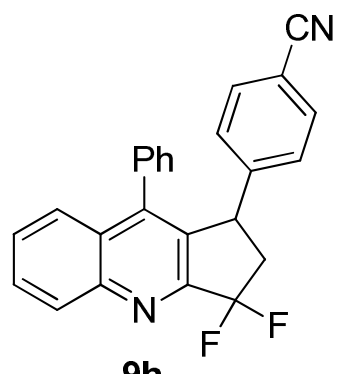

9h

$\begin{array}{lllllllllllllllllllllll}10 & 0 & -10 & -20 & -30 & -40 & -50 & -60 & -70 & -80 & -90 & -100 & -110 & -120 & -130 & -140 & -150 & -160 & -170 & -180 & -190 & -200 & -210\end{array}$ 

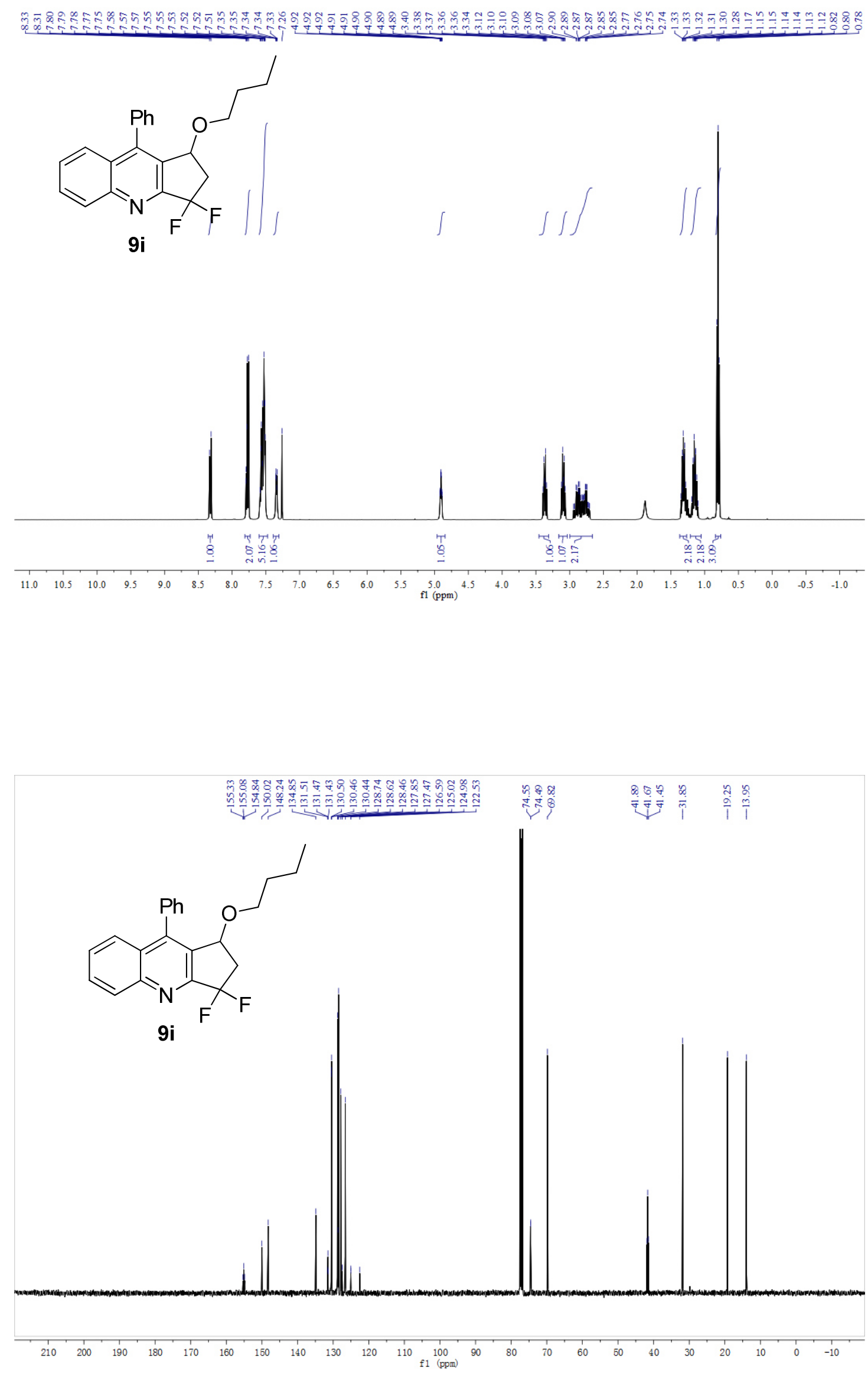


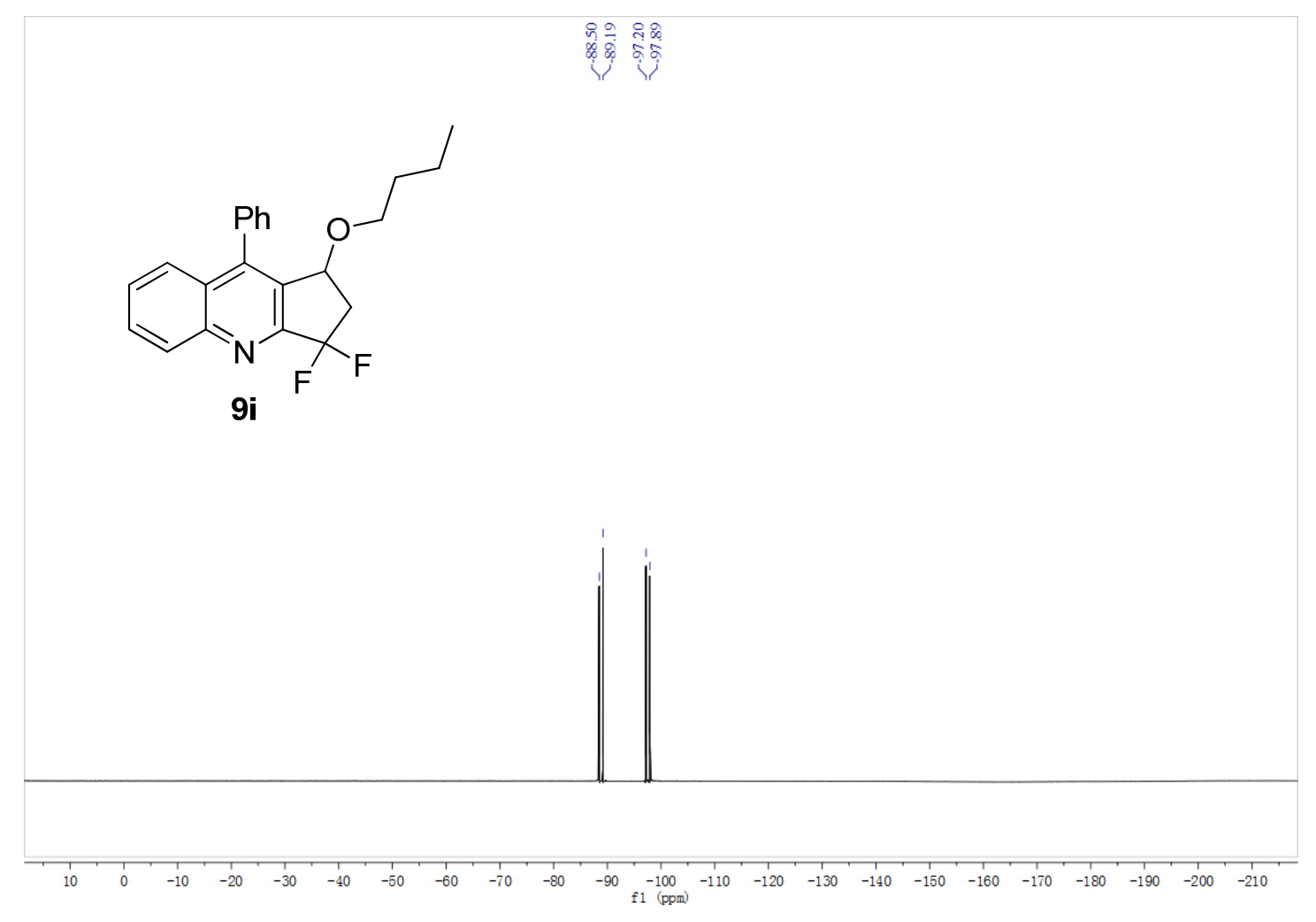

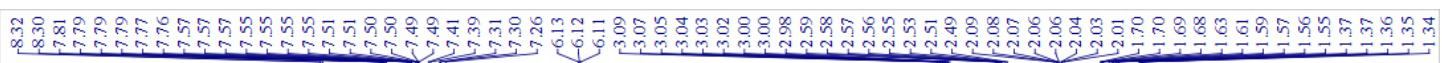<smiles>O=C1CCCN1C1CC(F)(F)c2nc3ccccc3c(-c3ccccc3)c21</smiles>

9j

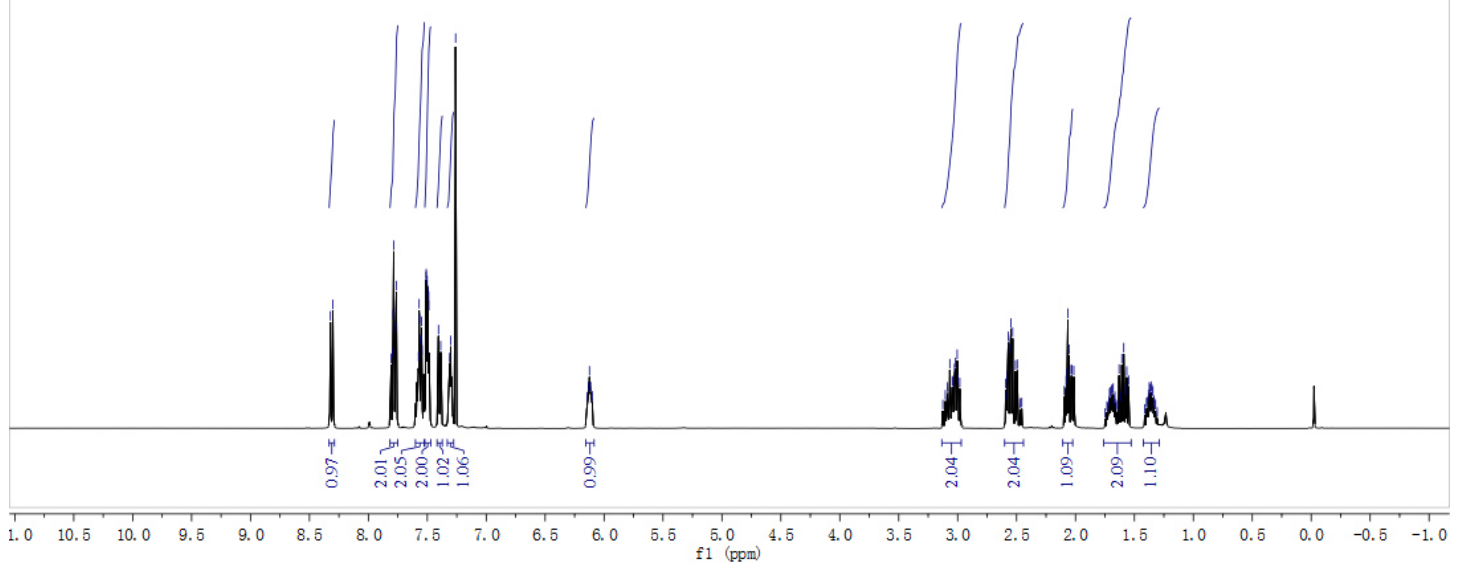



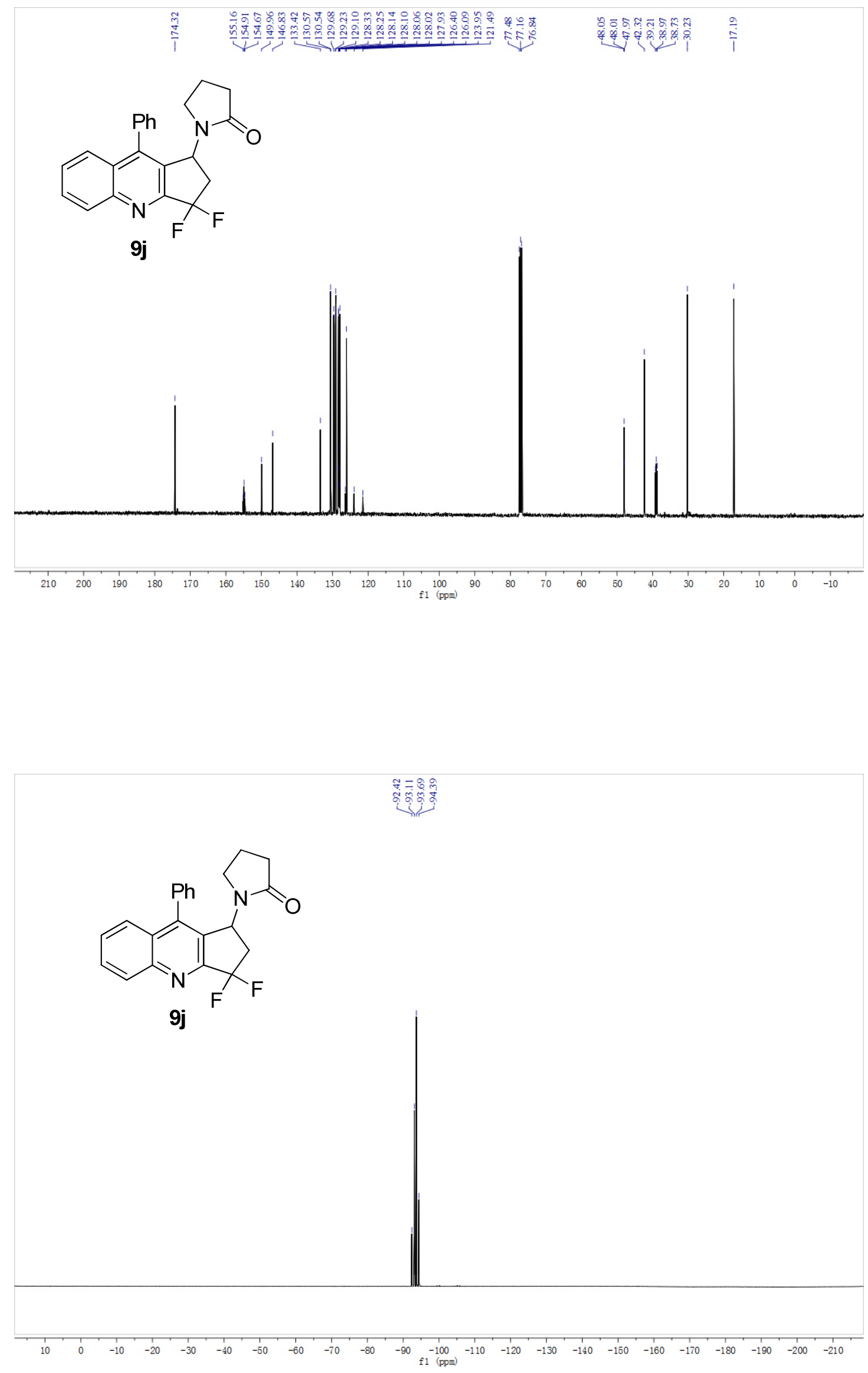


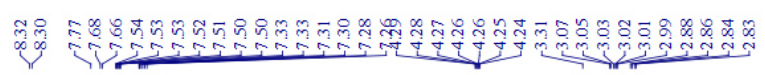

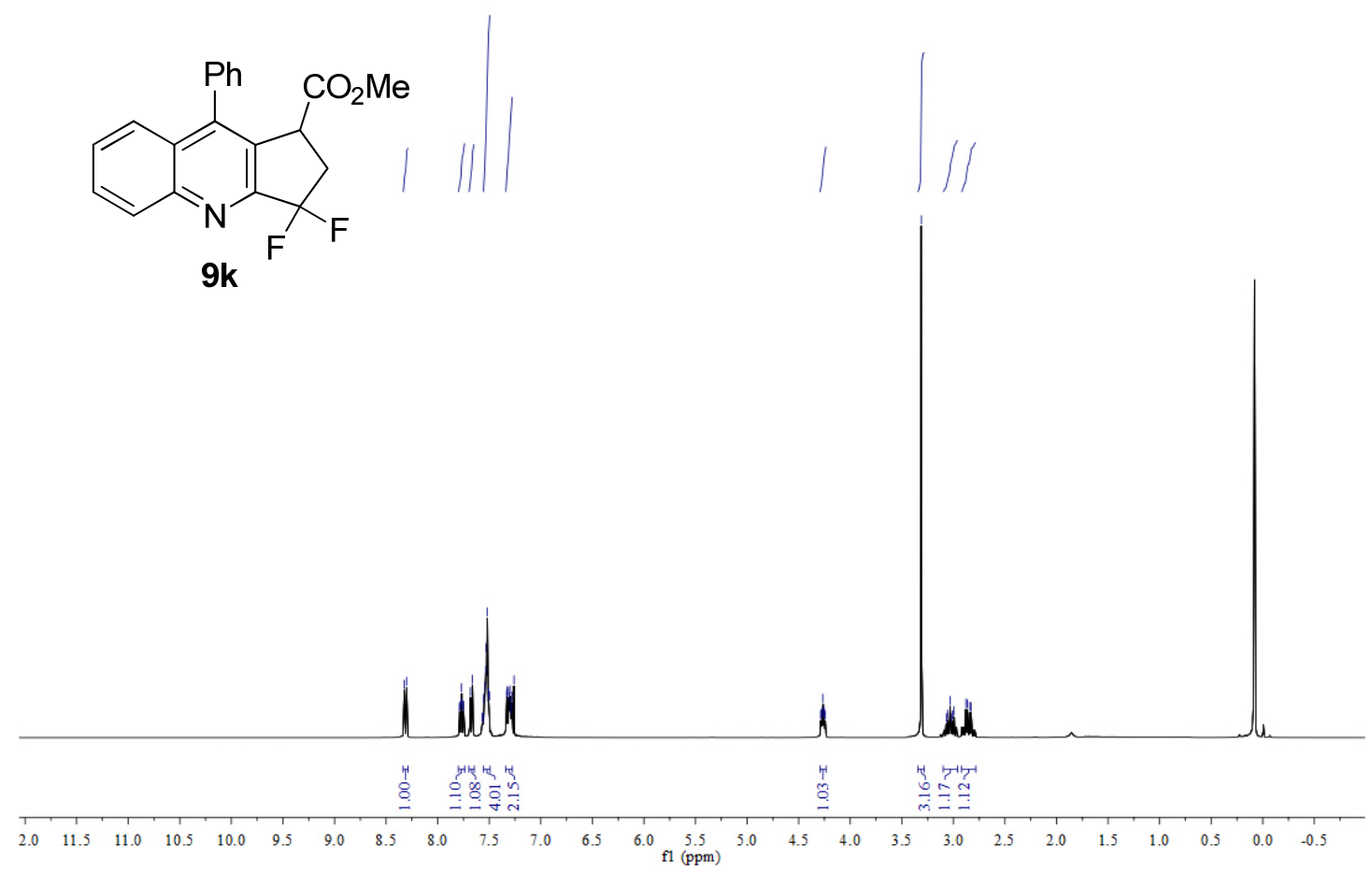

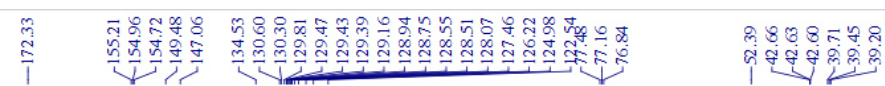<smiles>CC(=O)C1CC(F)(F)c2nc3ccccc3c(-c3ccccc3)c21</smiles>

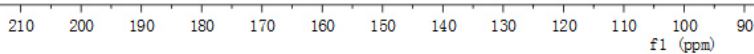




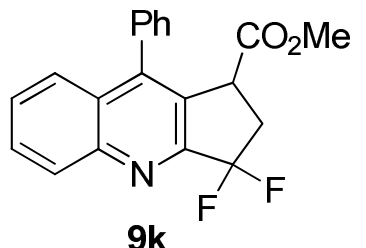

9k

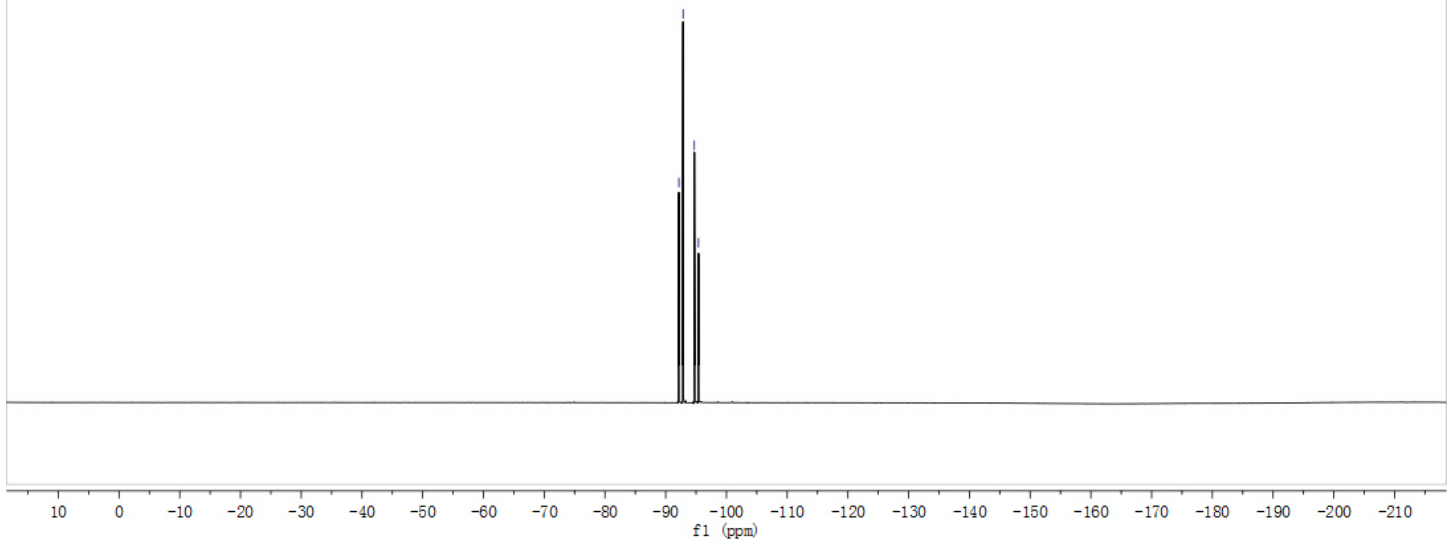

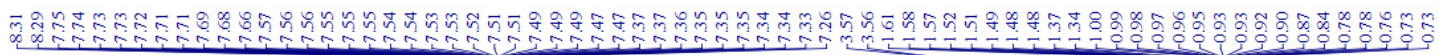<smiles>C#CC</smiles>

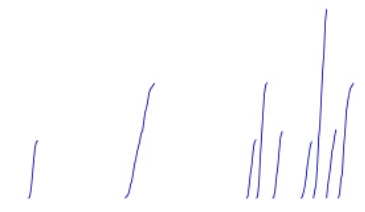

9l

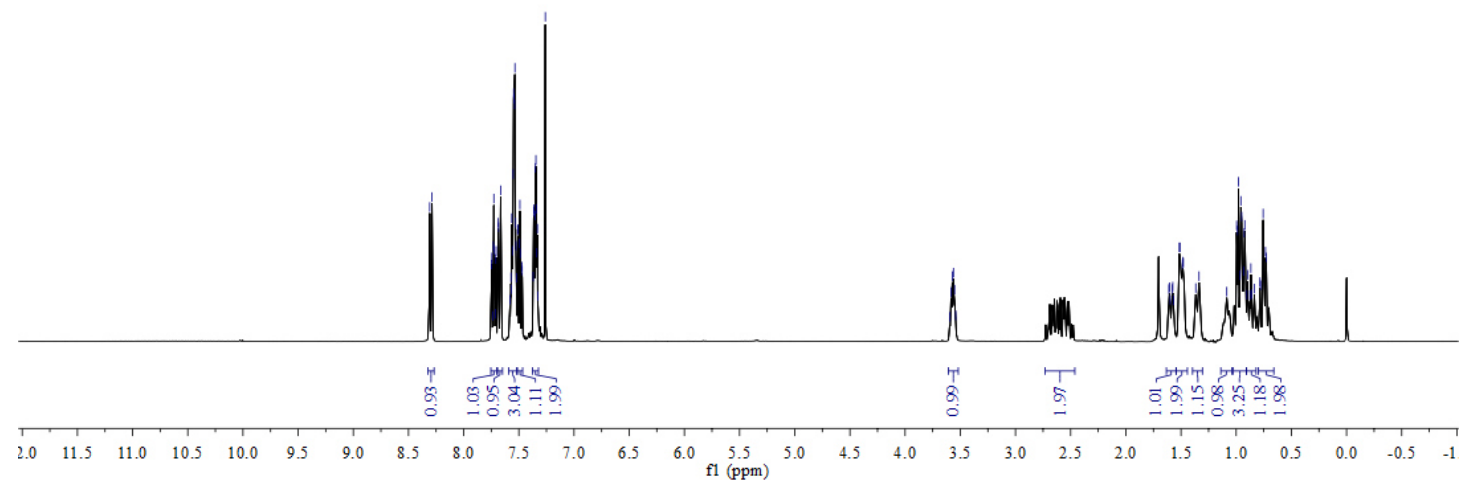



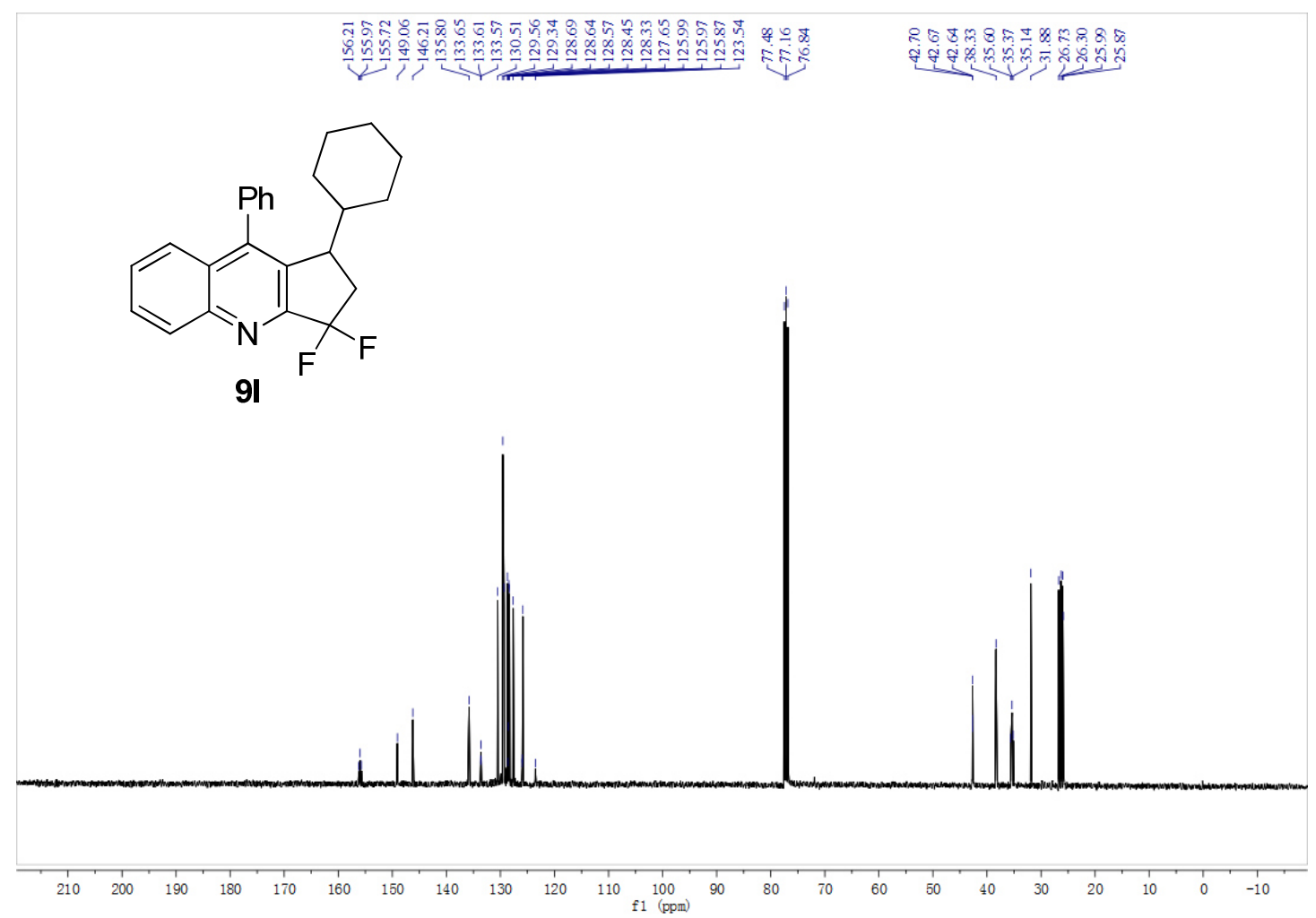

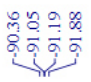
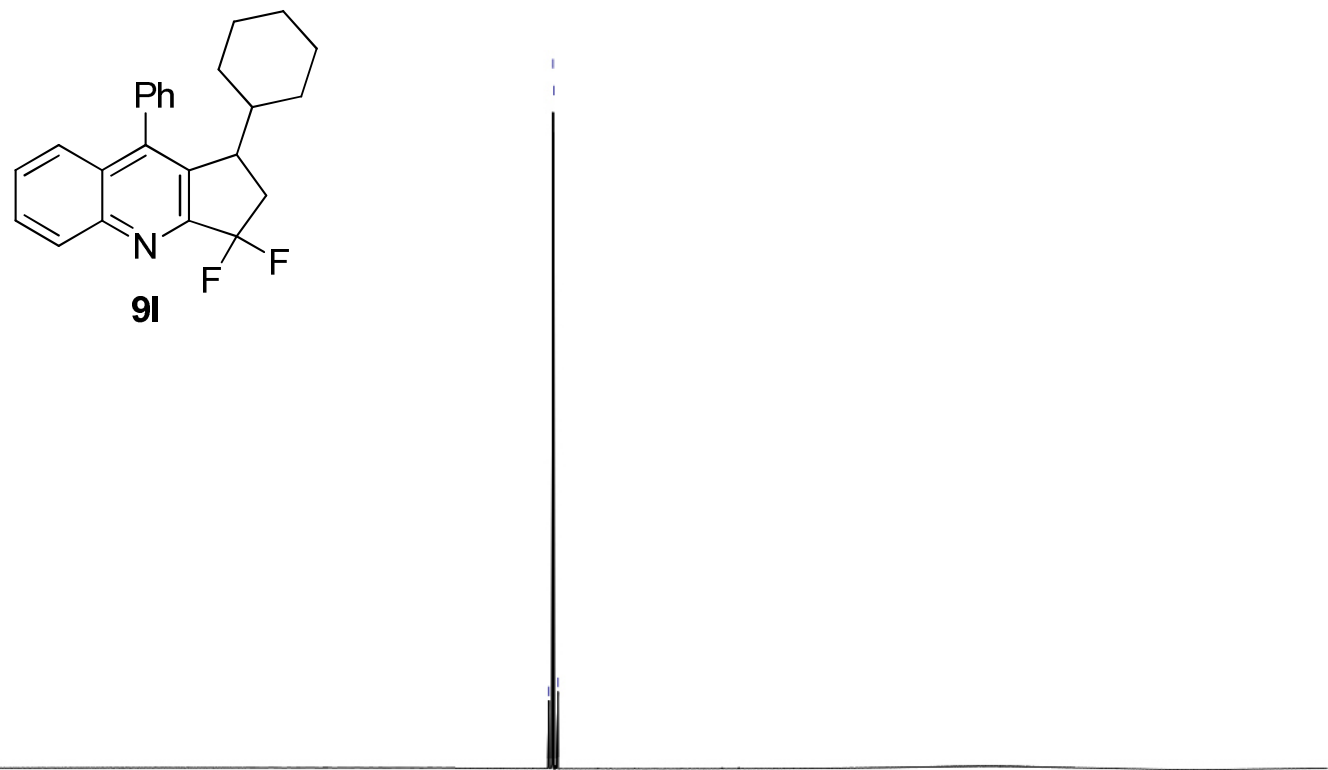

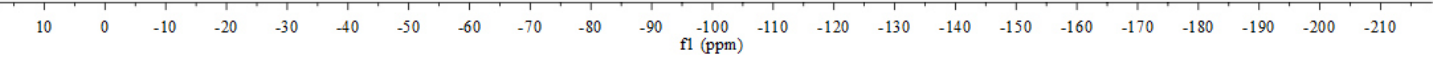




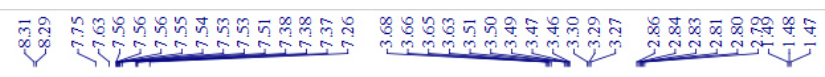
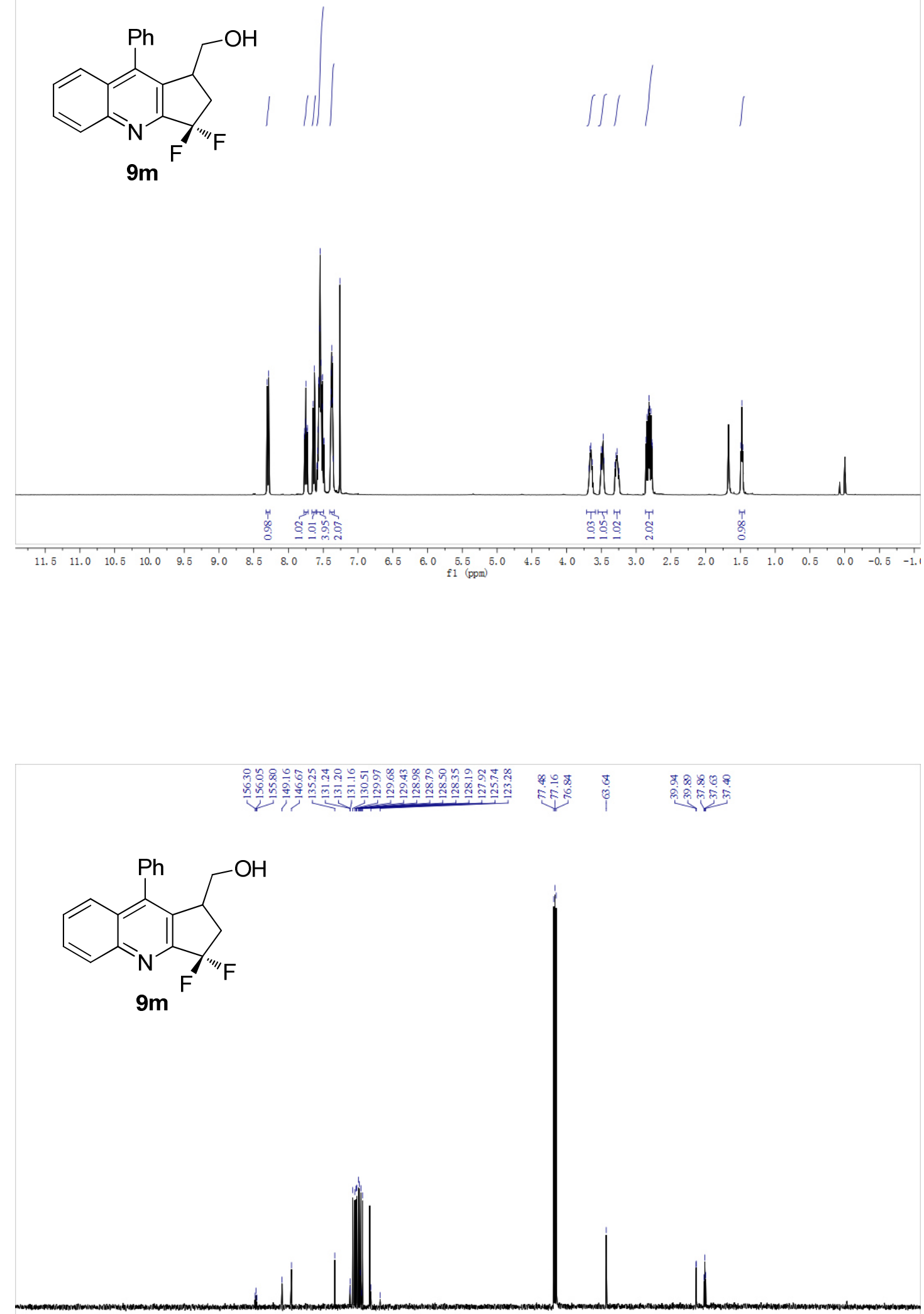

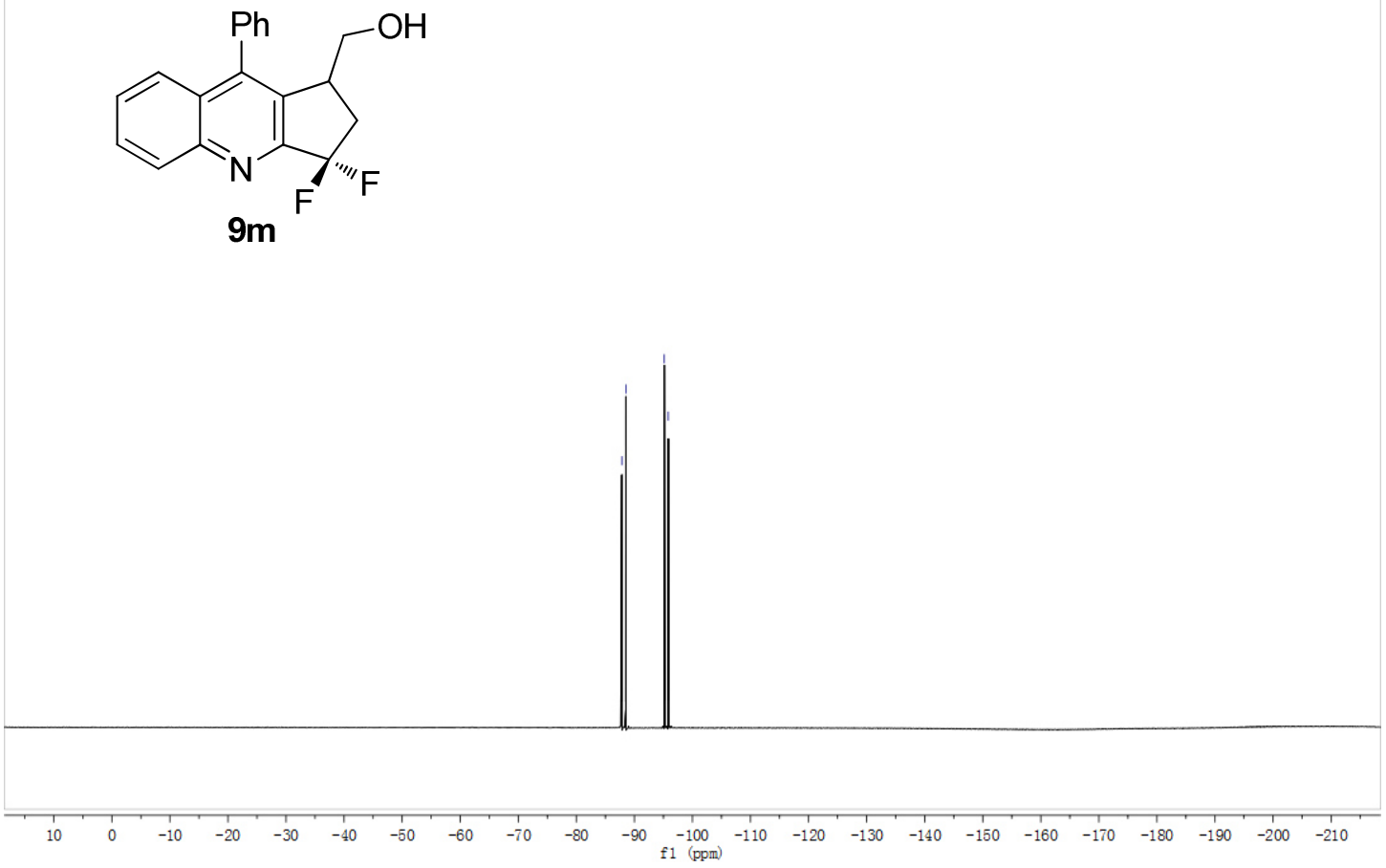

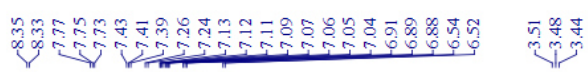

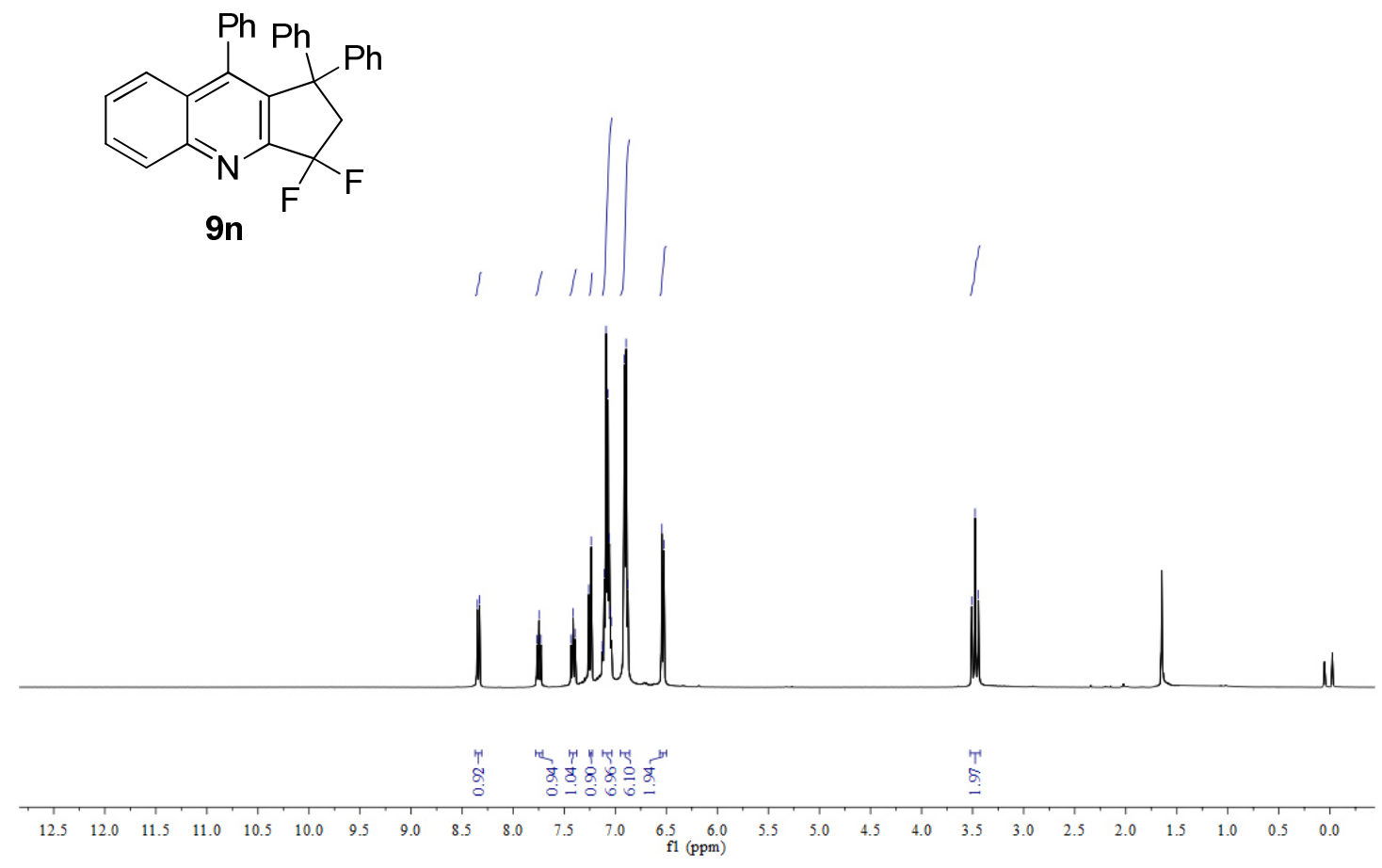




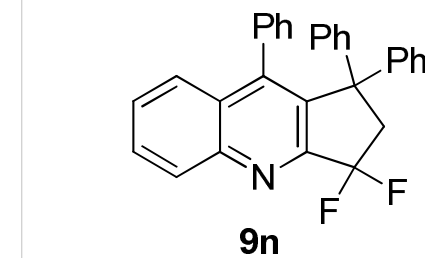

$\mathrm{Ph}$
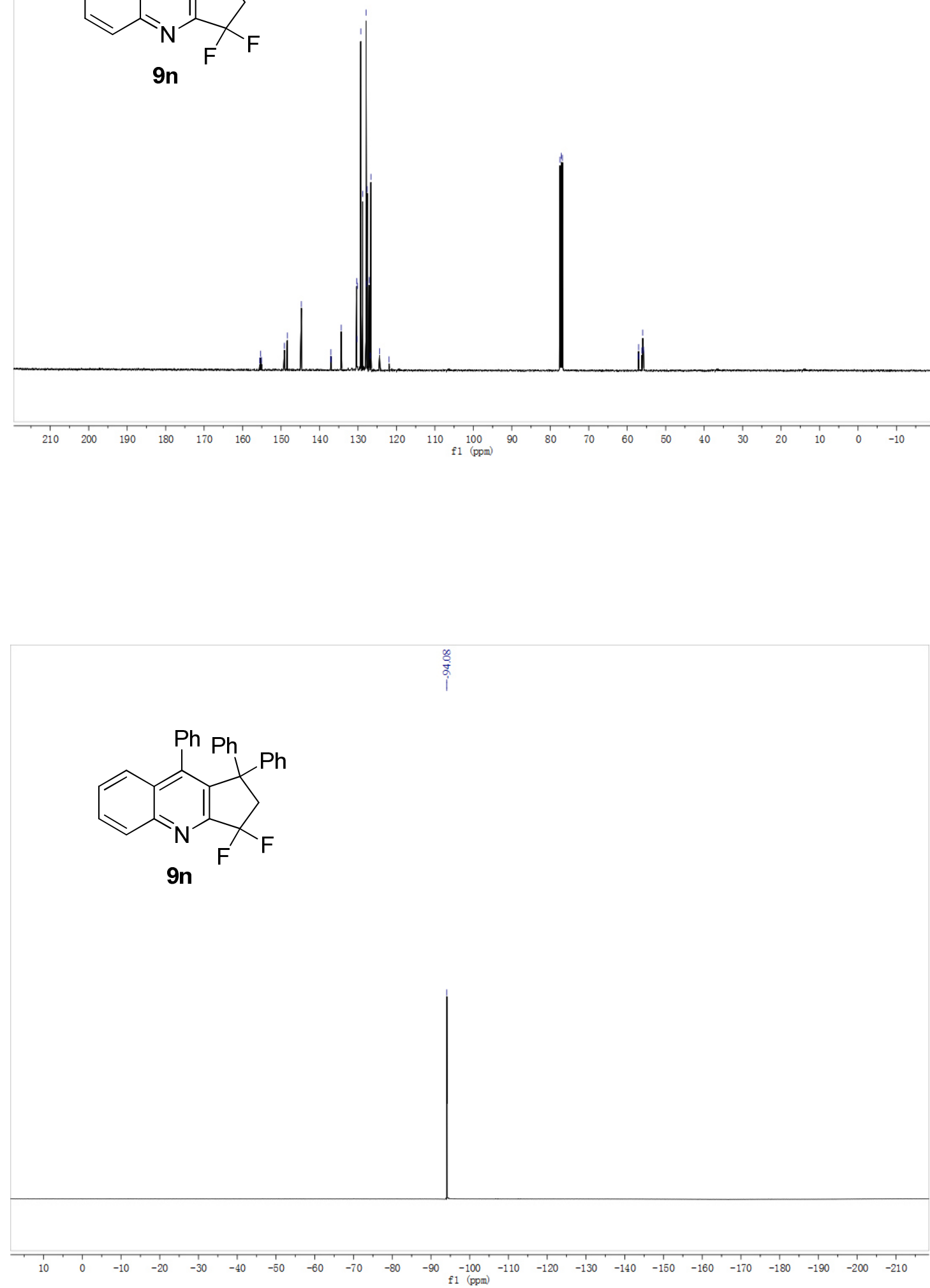


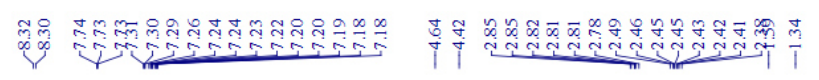
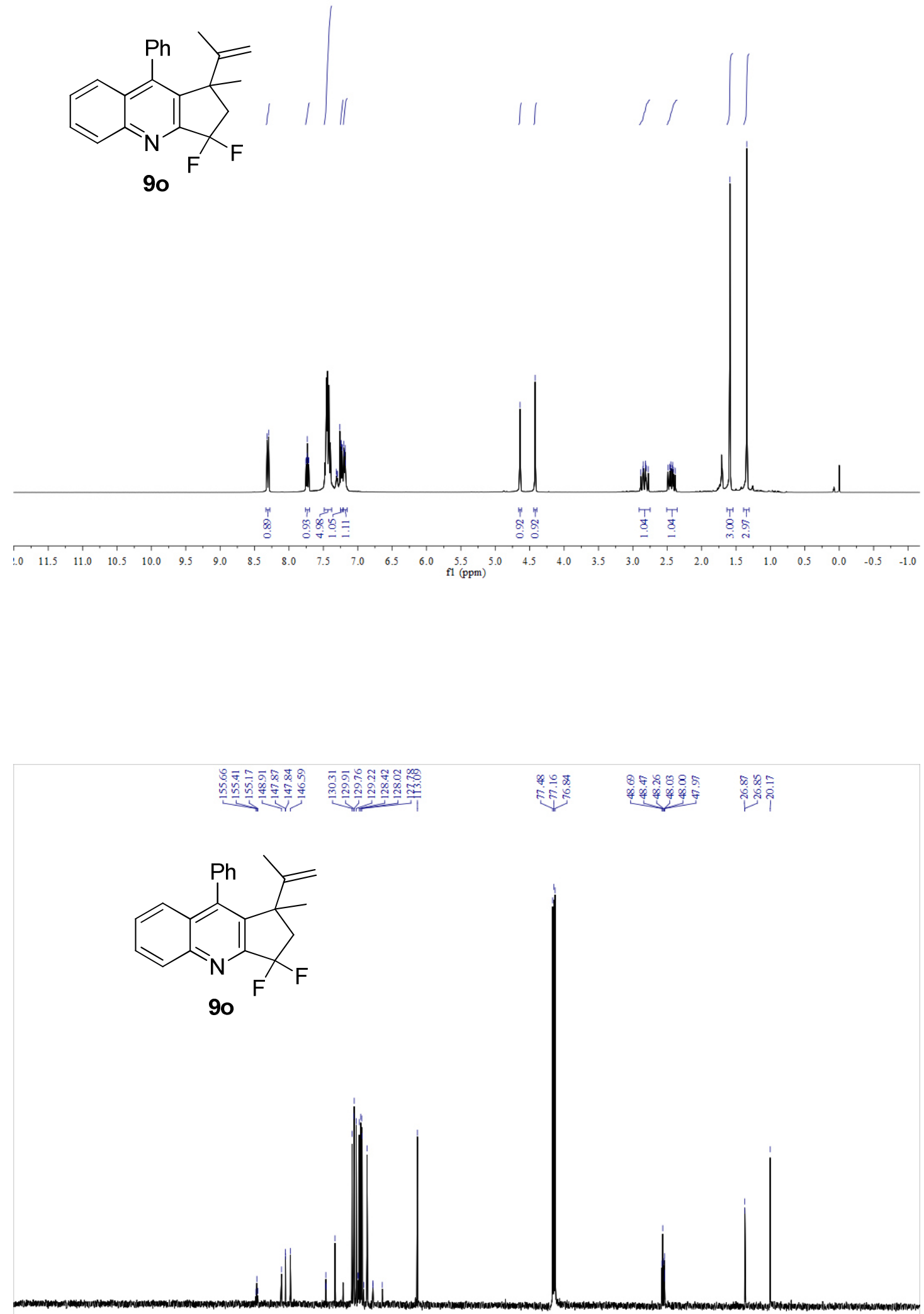

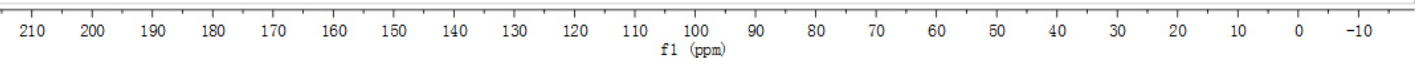




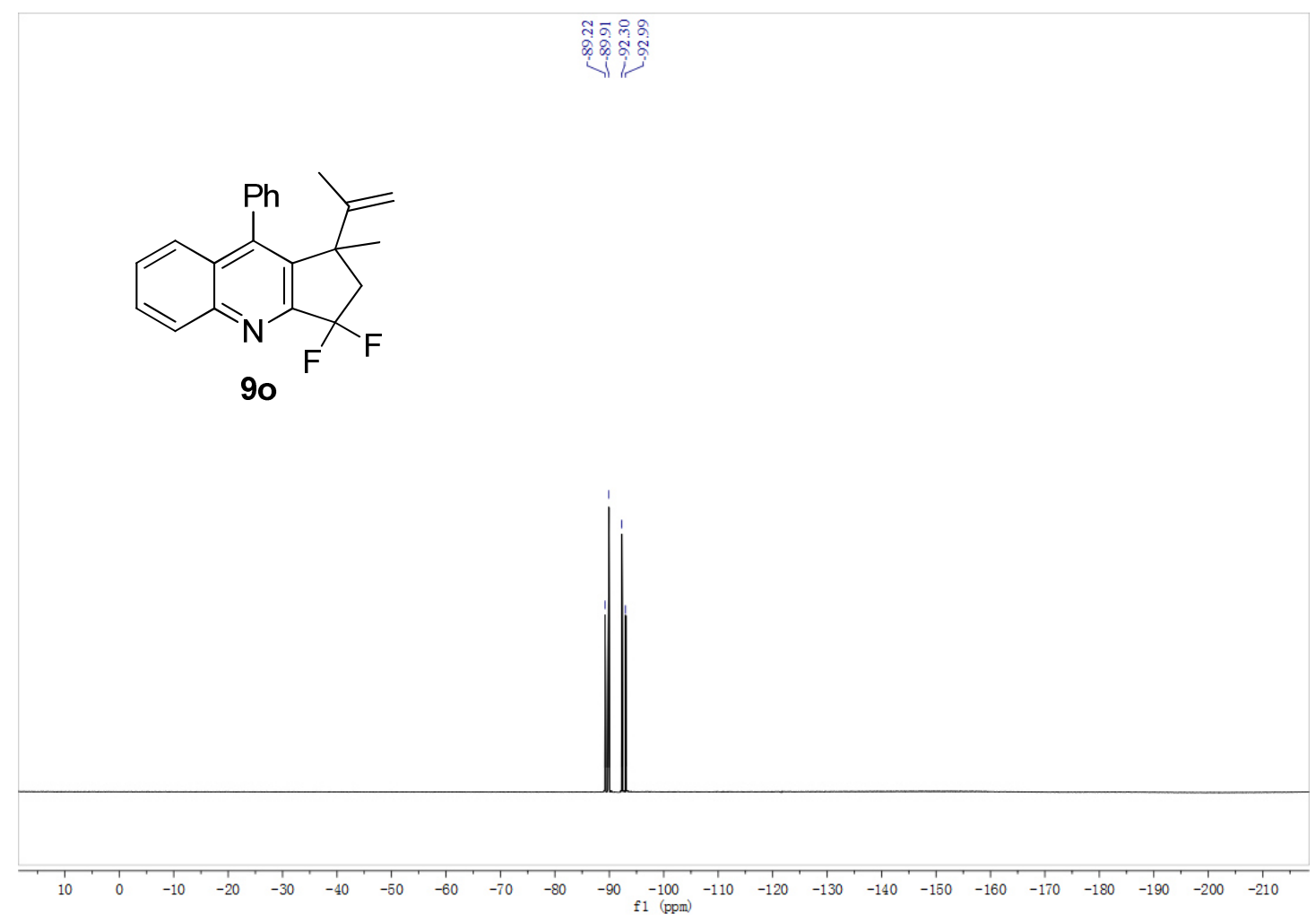

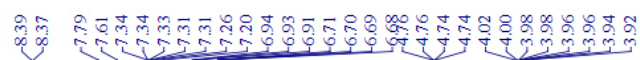
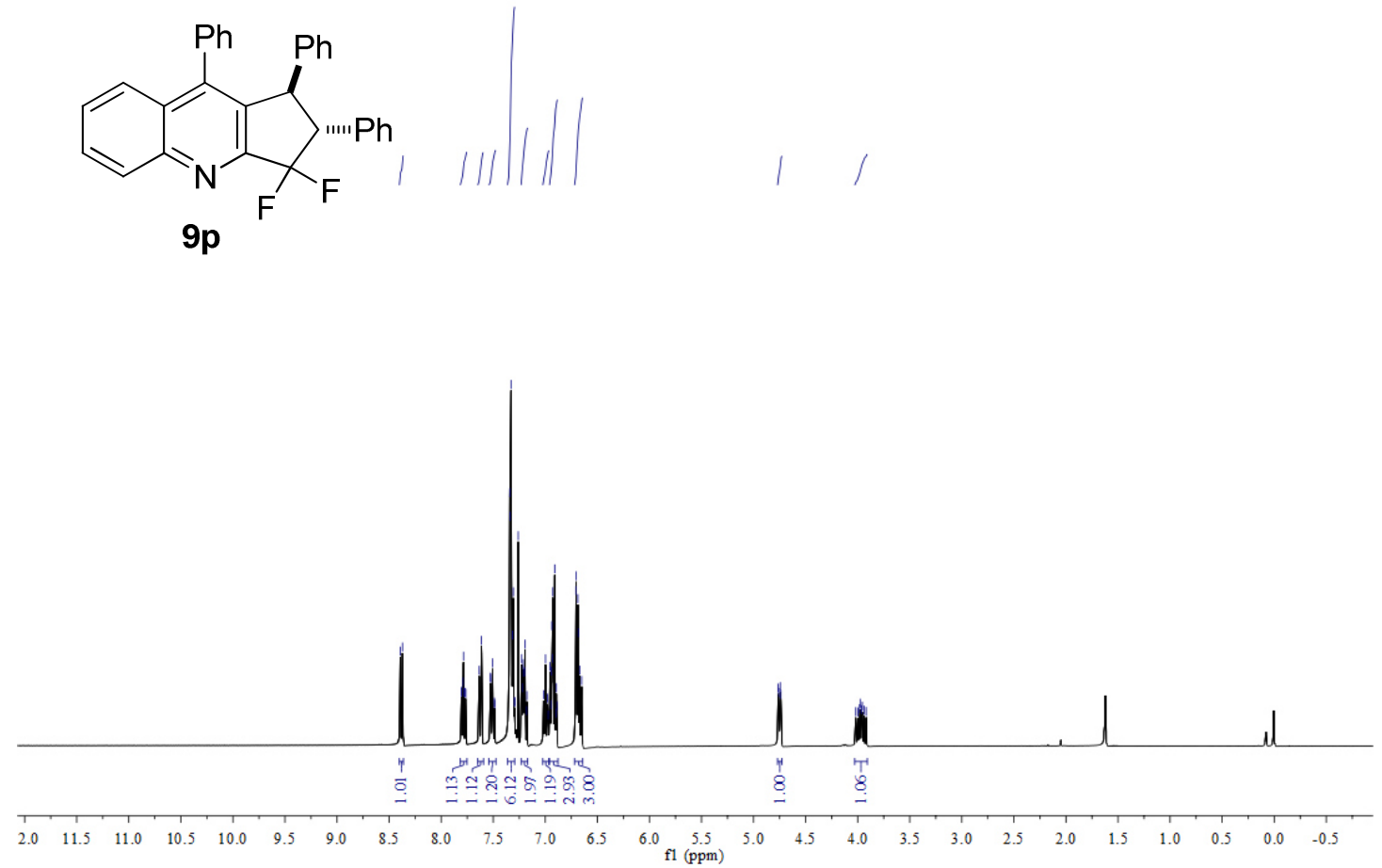

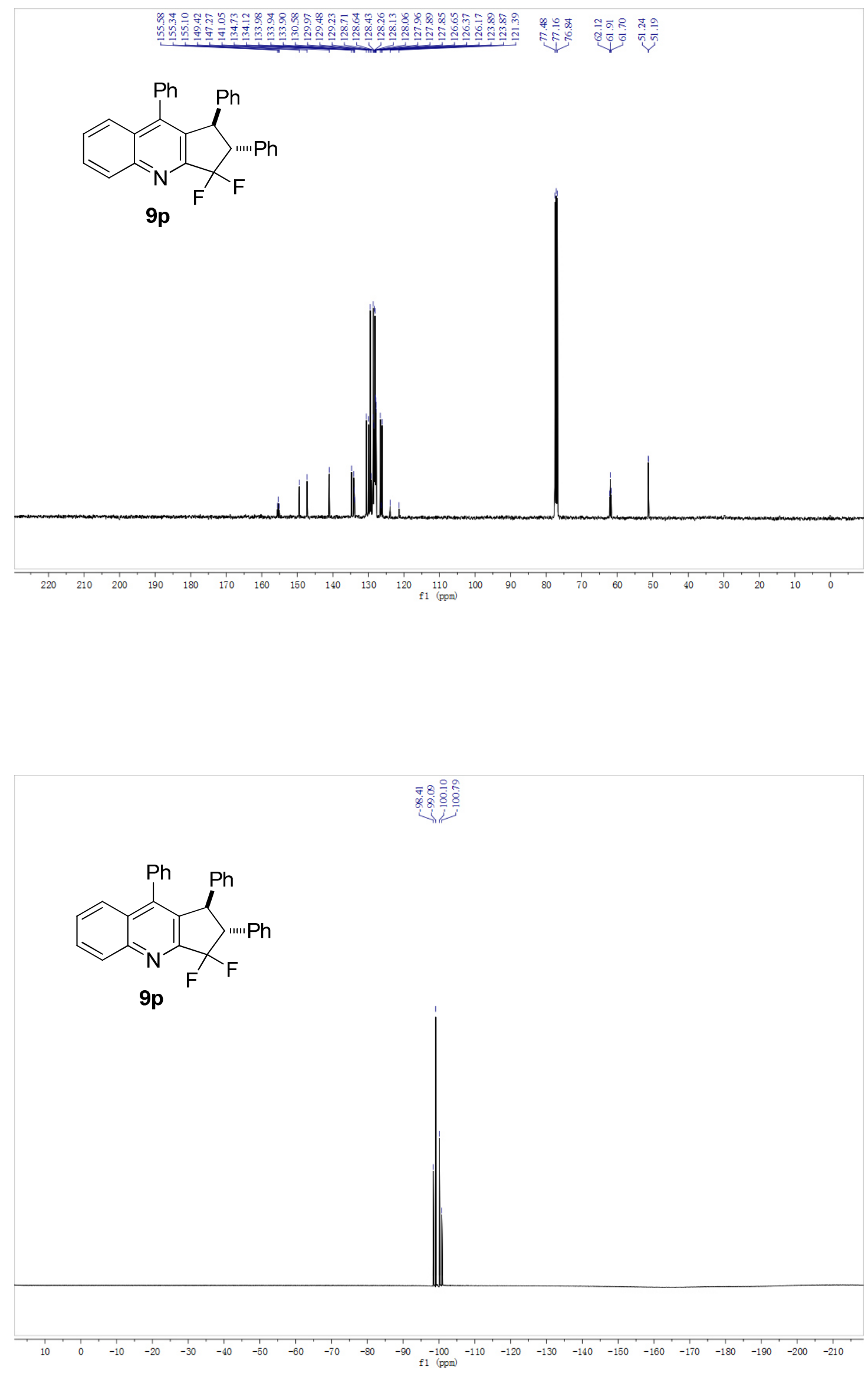


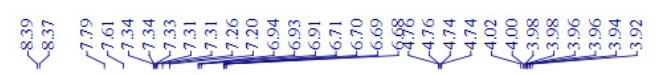
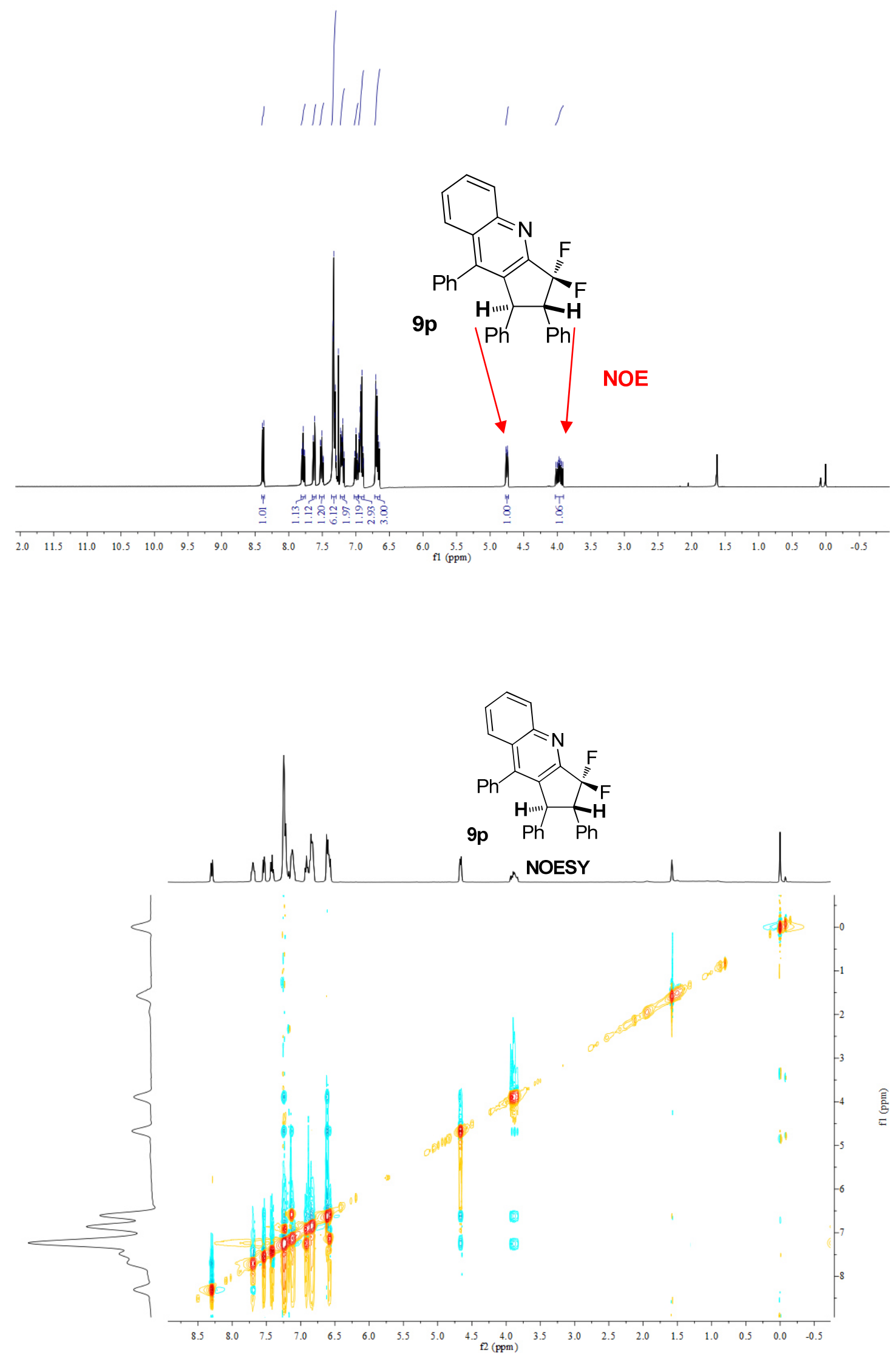

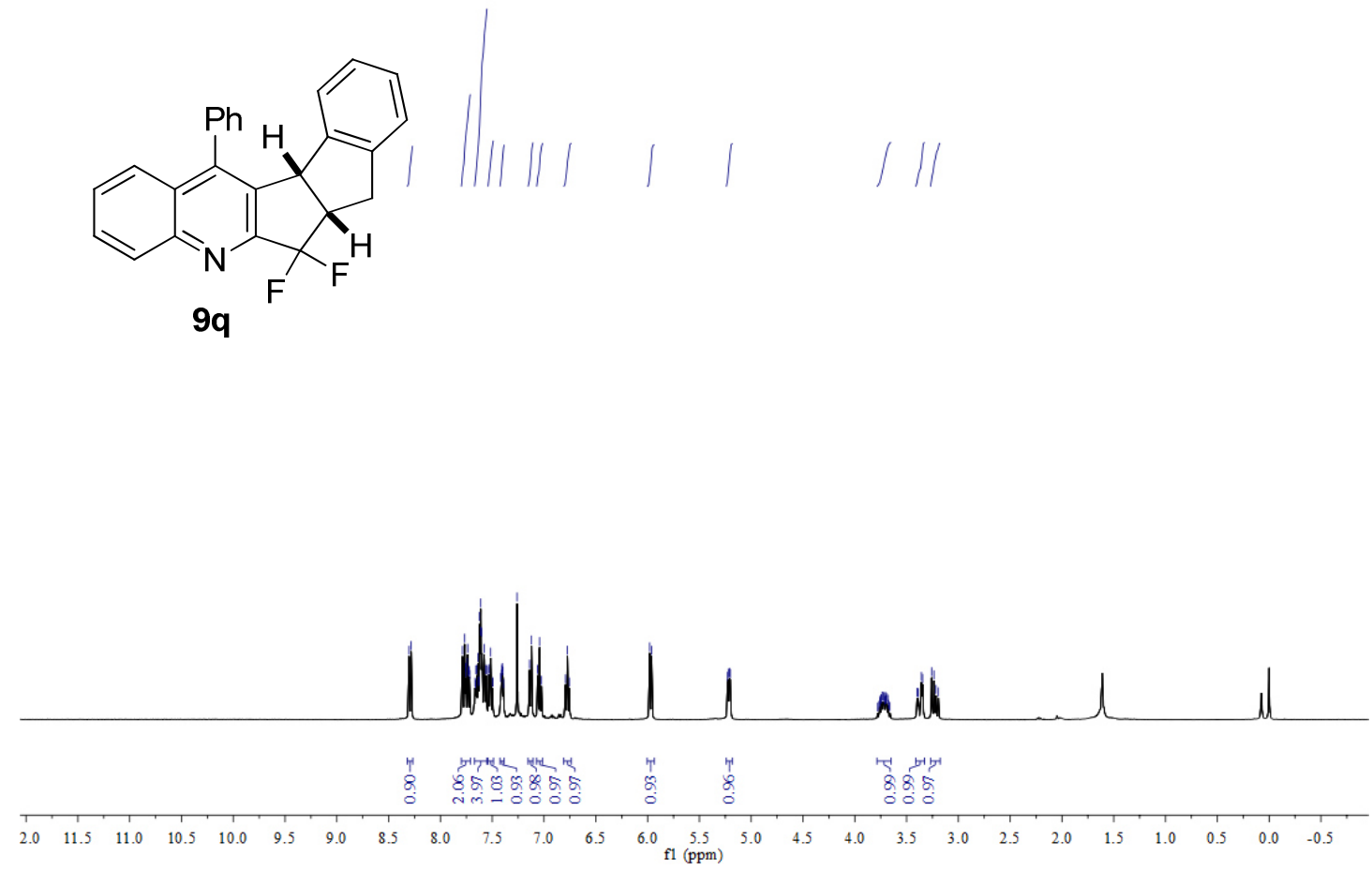

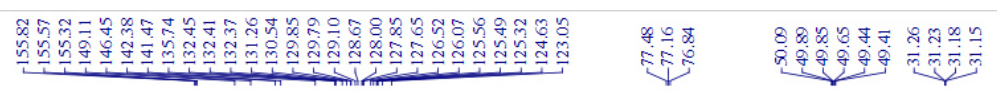<smiles>FC(F)(F)c1nc2ccccc2c(-c2ccccc2)c1C1CCc2ccccc21</smiles>

9q

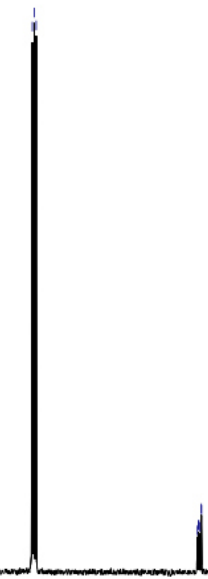

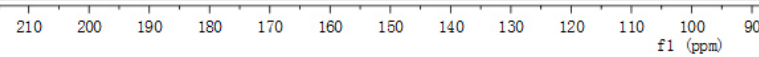


<smiles>FC1(F)c2nc3ccccc3c(-c3ccccc3)c2[C@H]2Cc3ccccc3CC21</smiles>
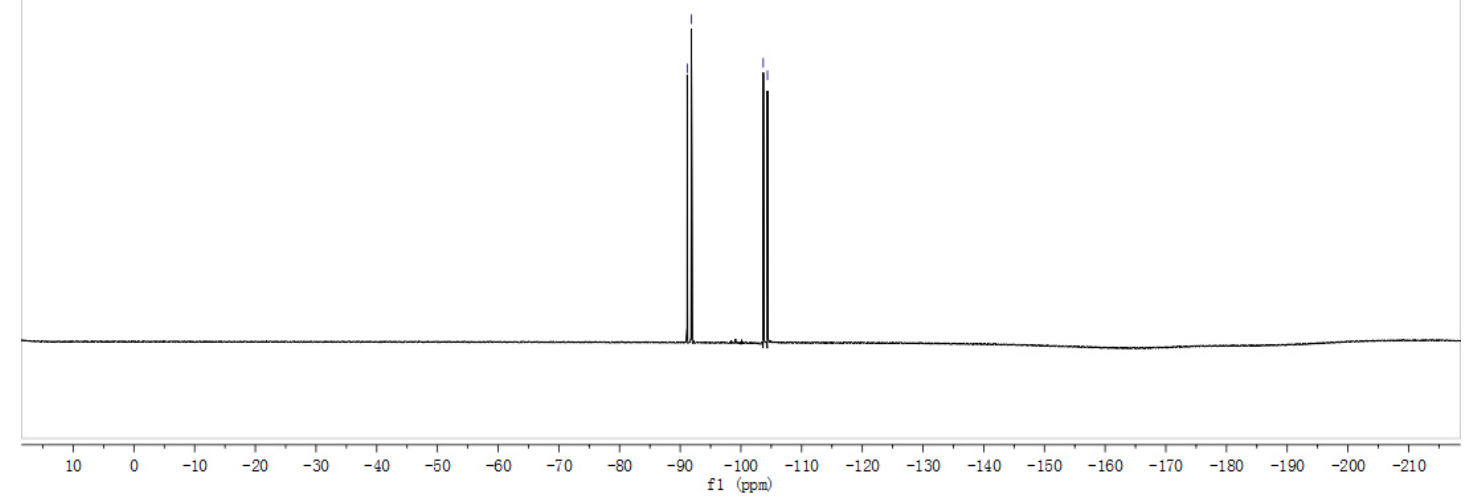

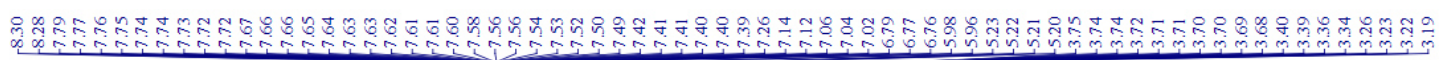

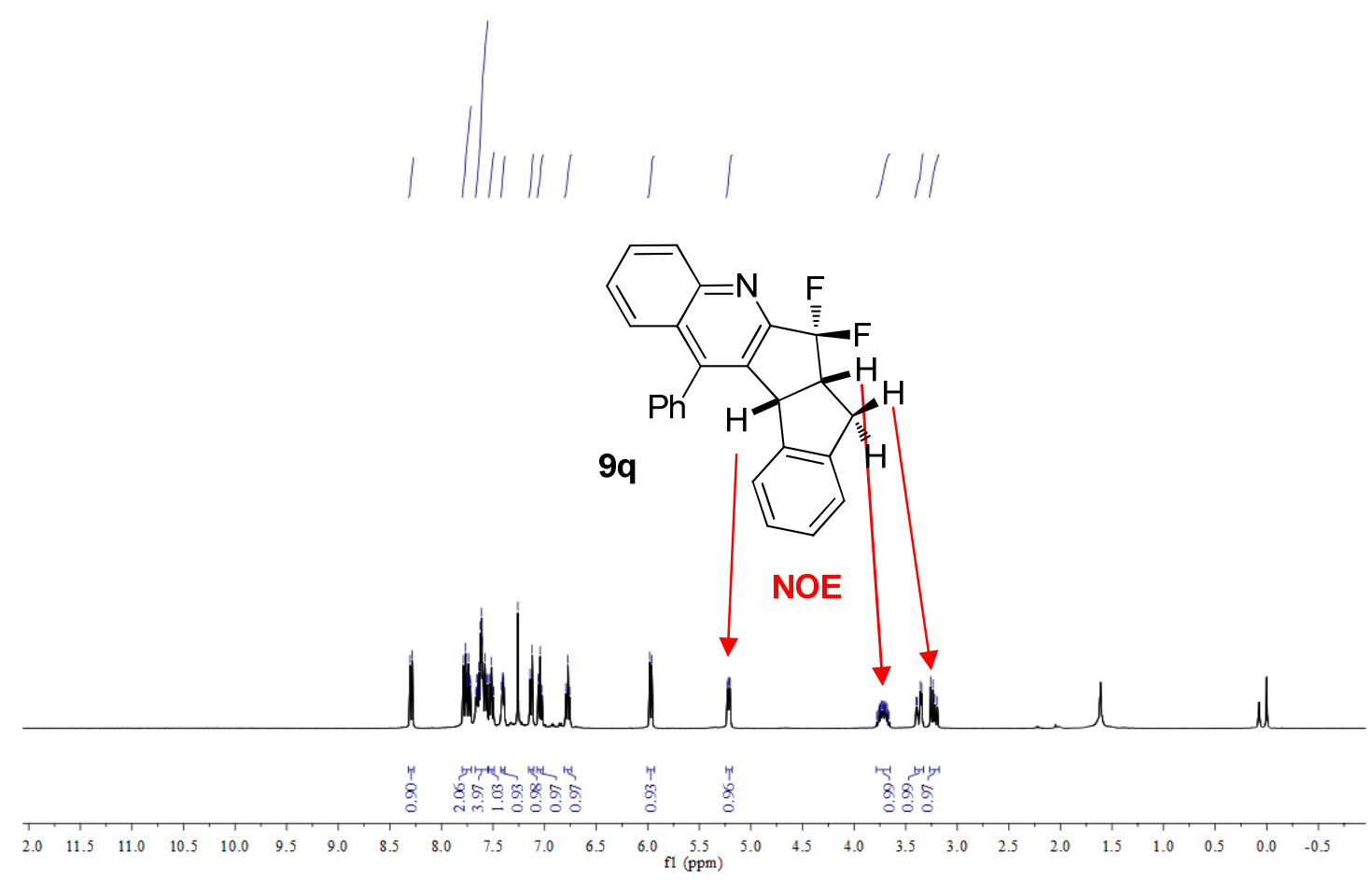




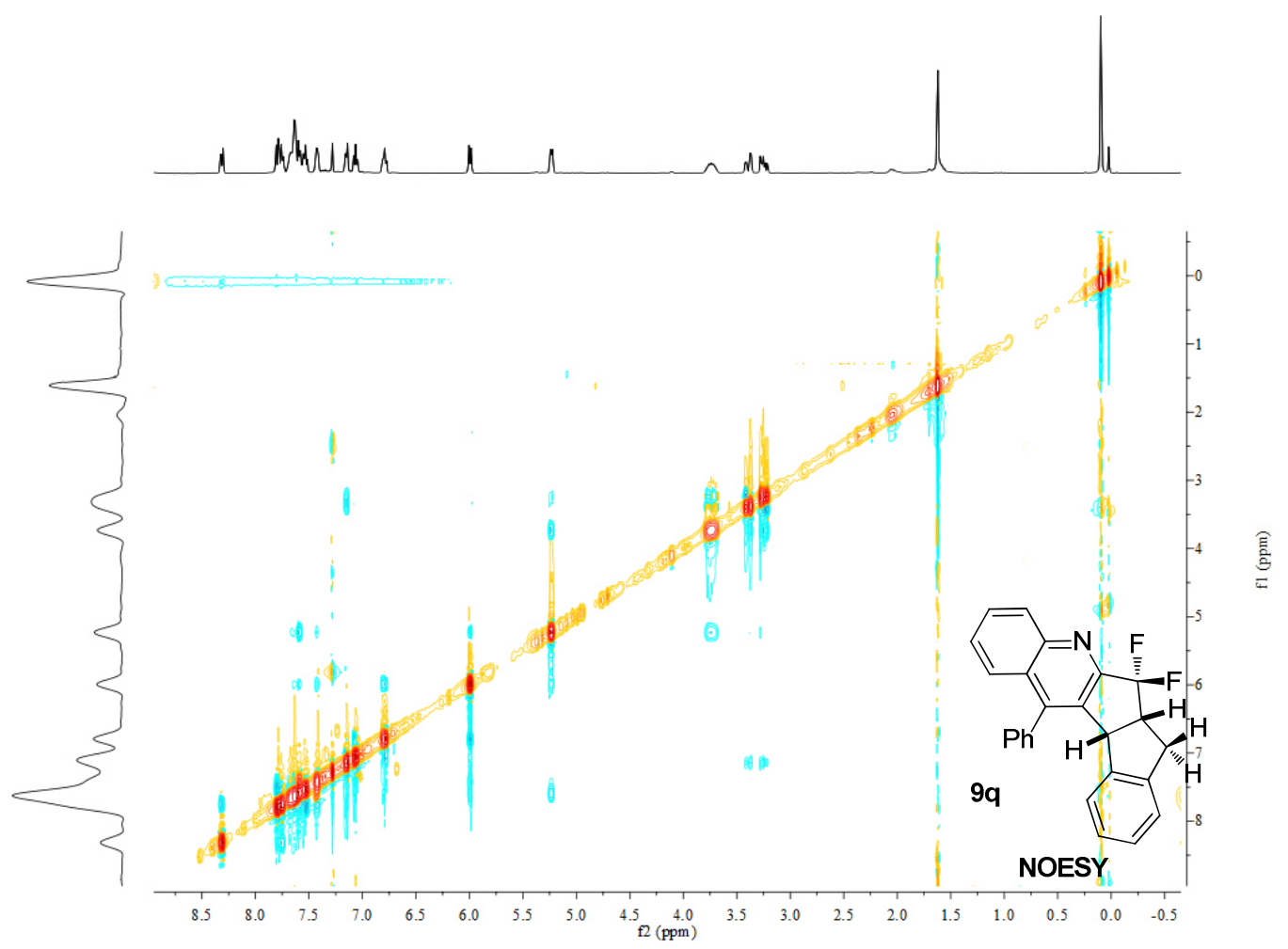

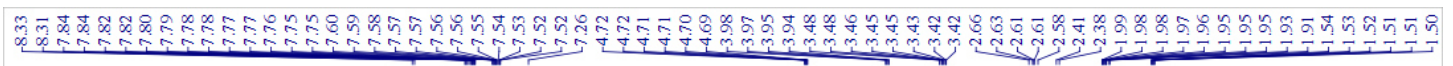

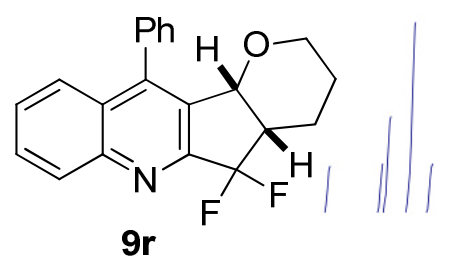

$$
111111
$$

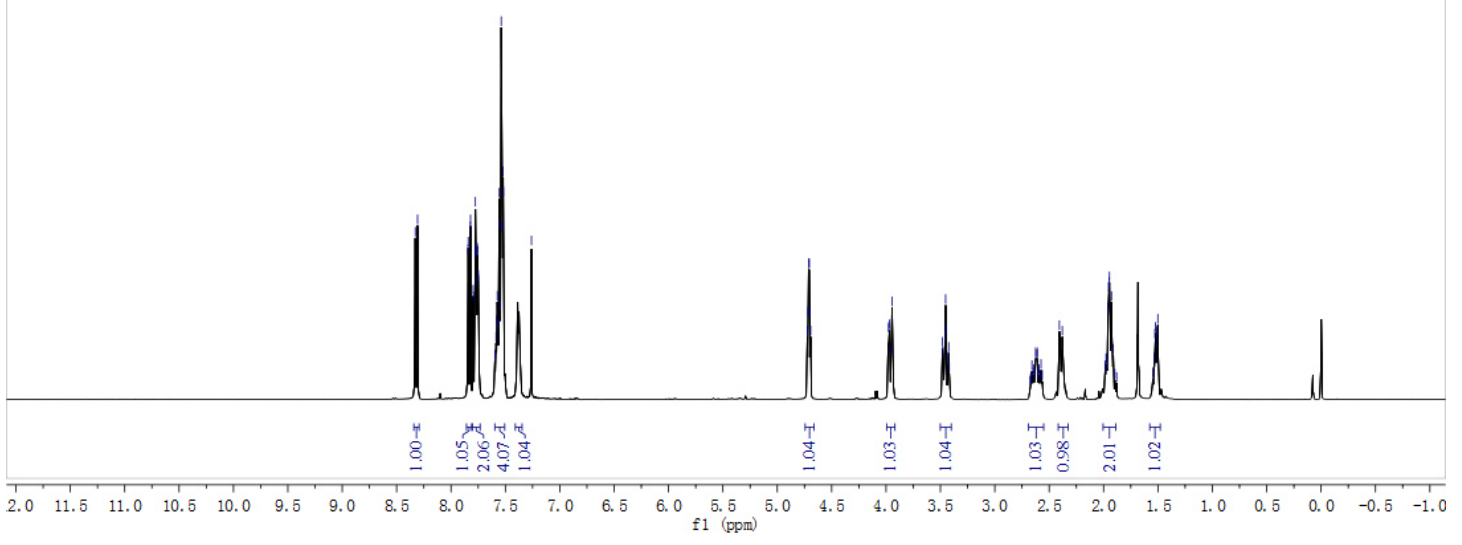

S86 

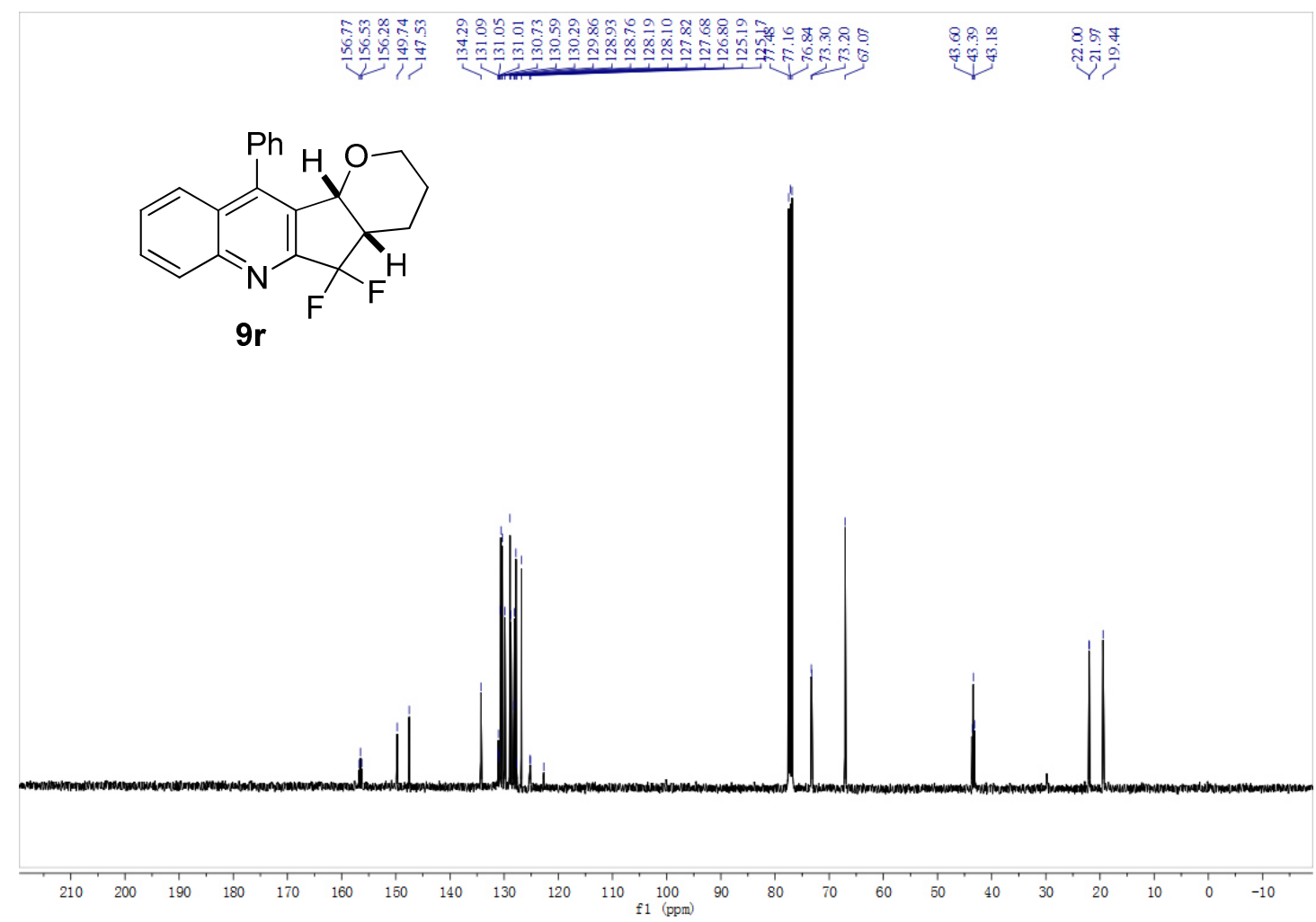

ํํำ<smiles>FC1(F)c2nc3ccccc3c(-c3ccccc3)c2C2(CCCCO2)C1(F)F</smiles>

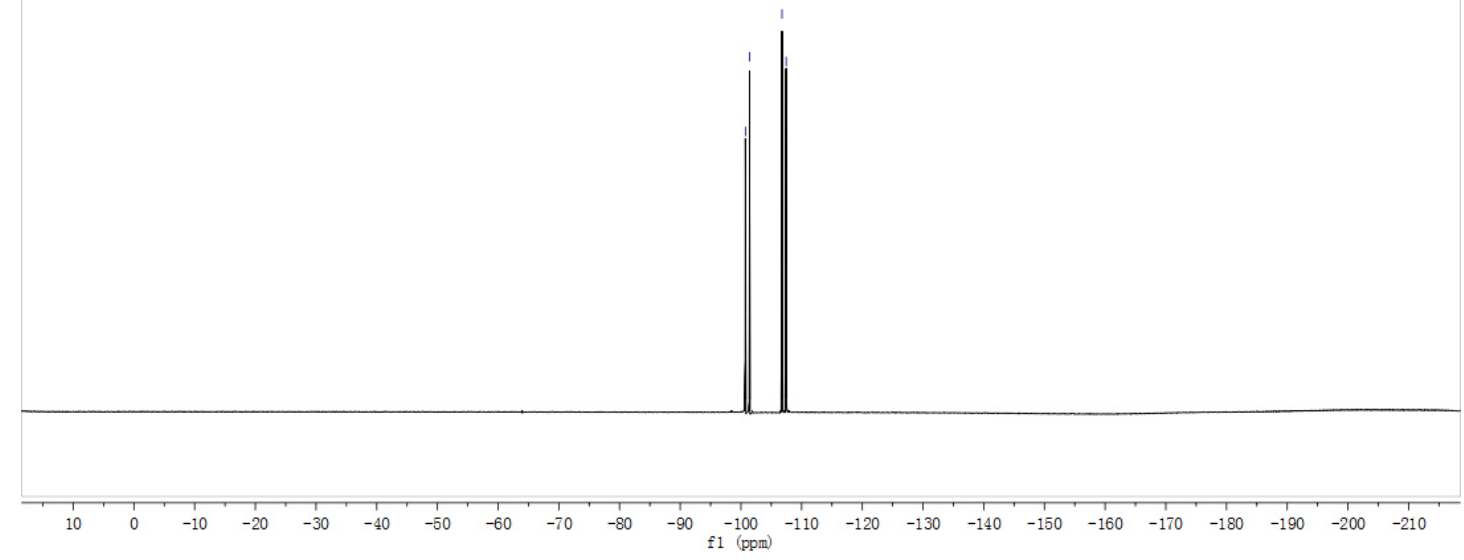



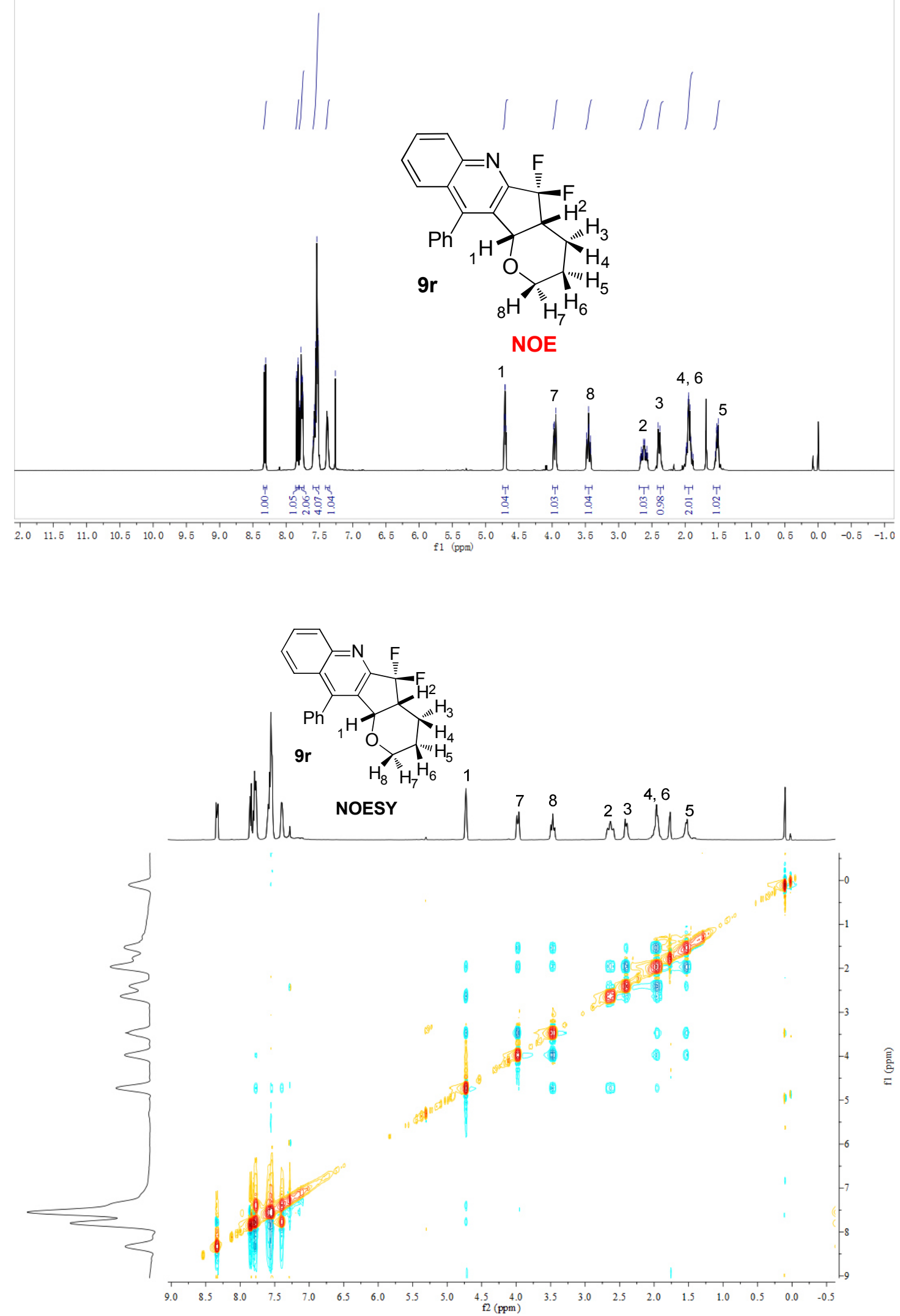
<smiles>C#CC1(F)c2nc3ccccc3c(-c3ccccc3)c2C2CCC1C2</smiles>

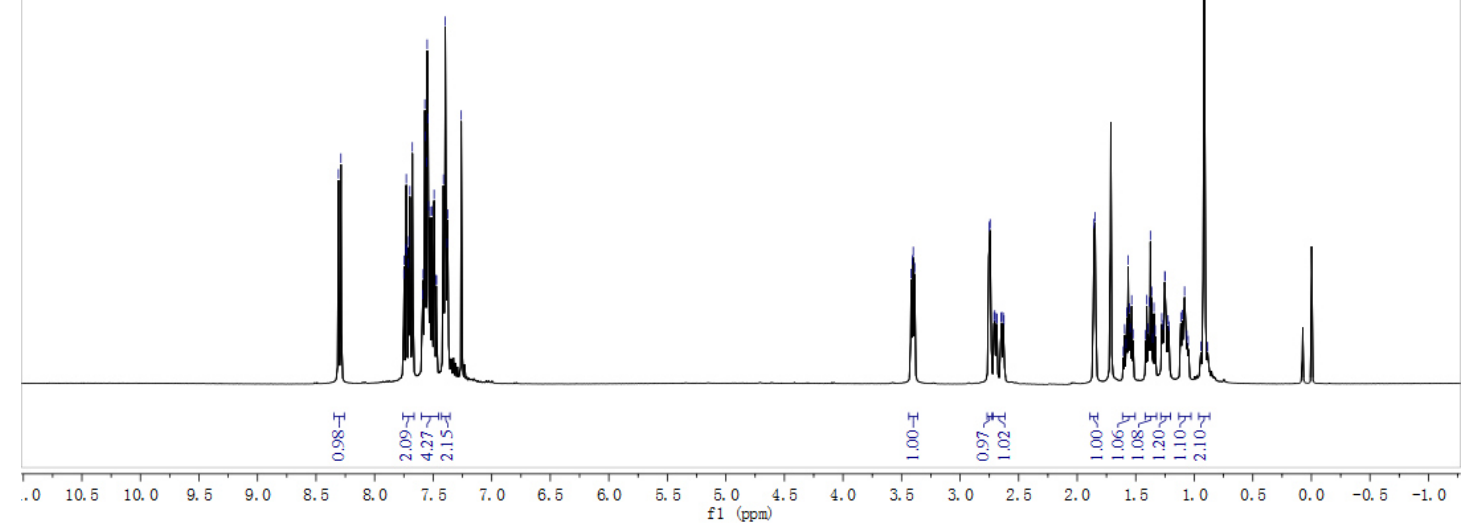

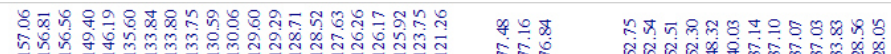

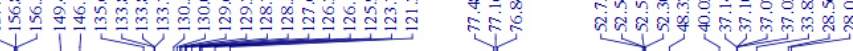<smiles></smiles>

9s

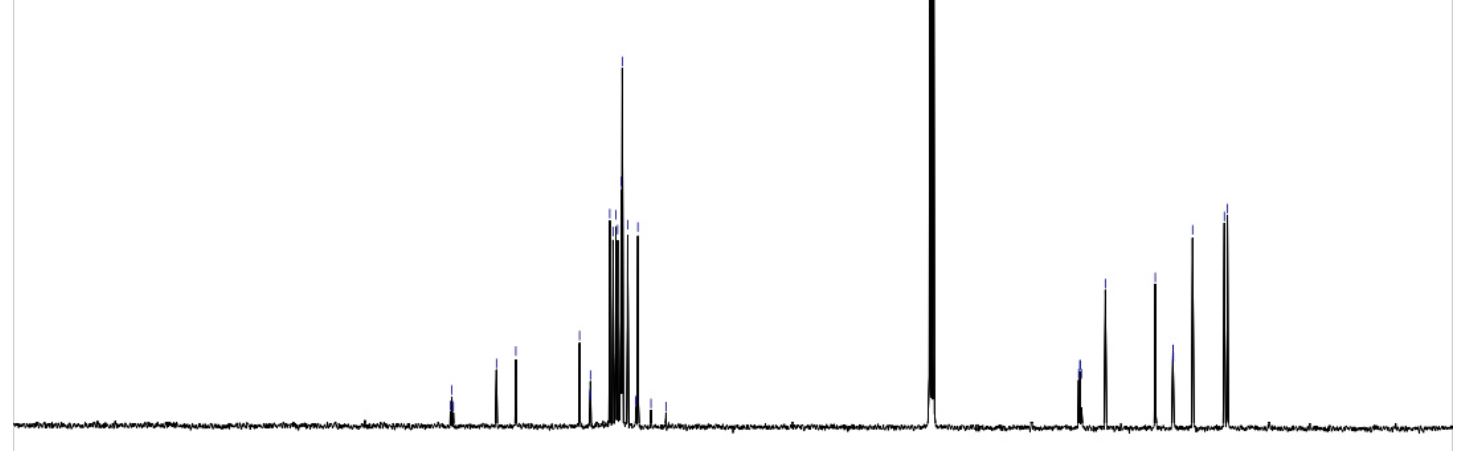

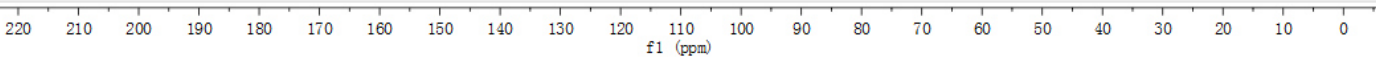


<smiles>FC1(F)CC2(CC3CCC2C3)c2c1nc1ccccc1c2-c1ccccc1</smiles>

9s
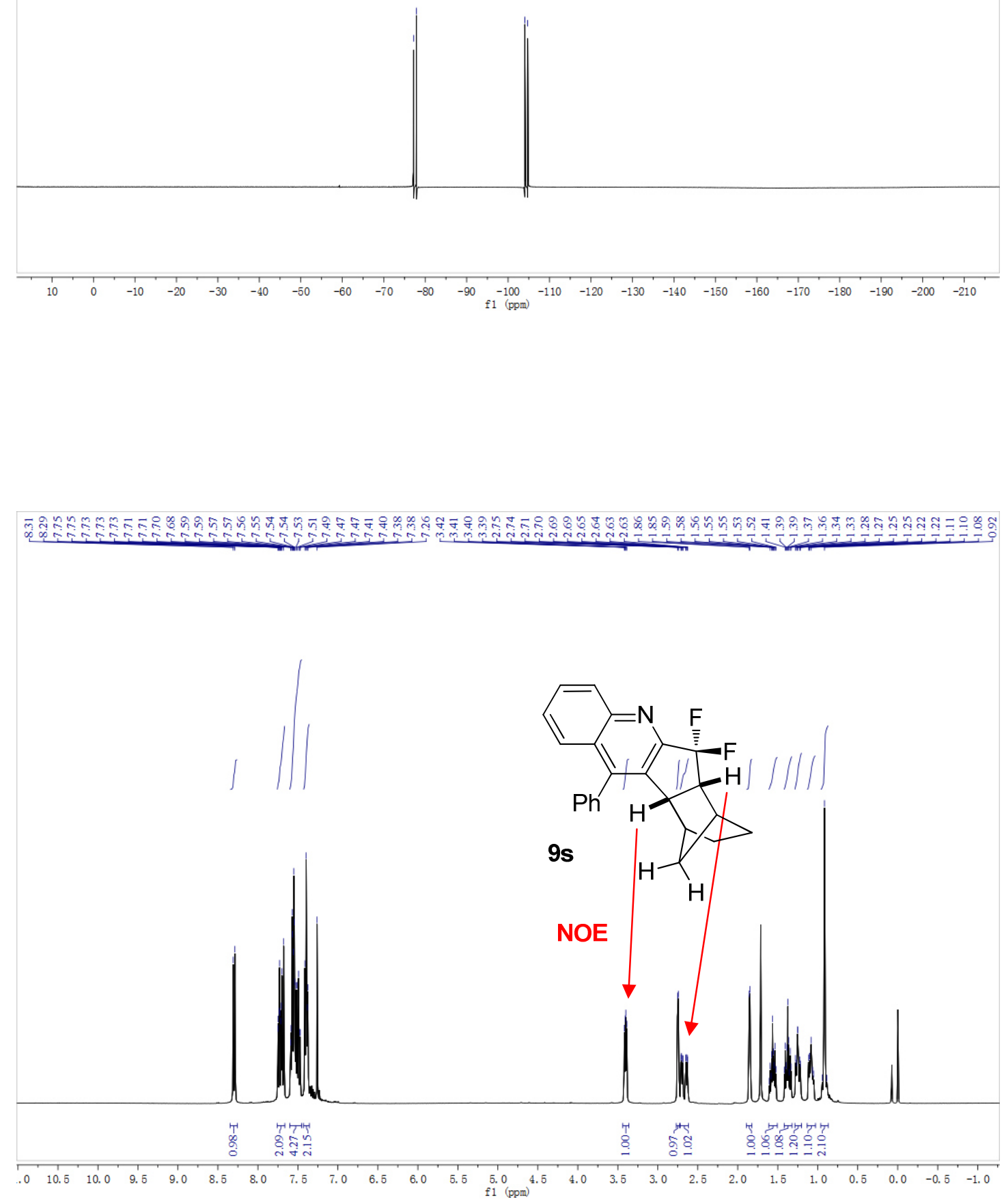


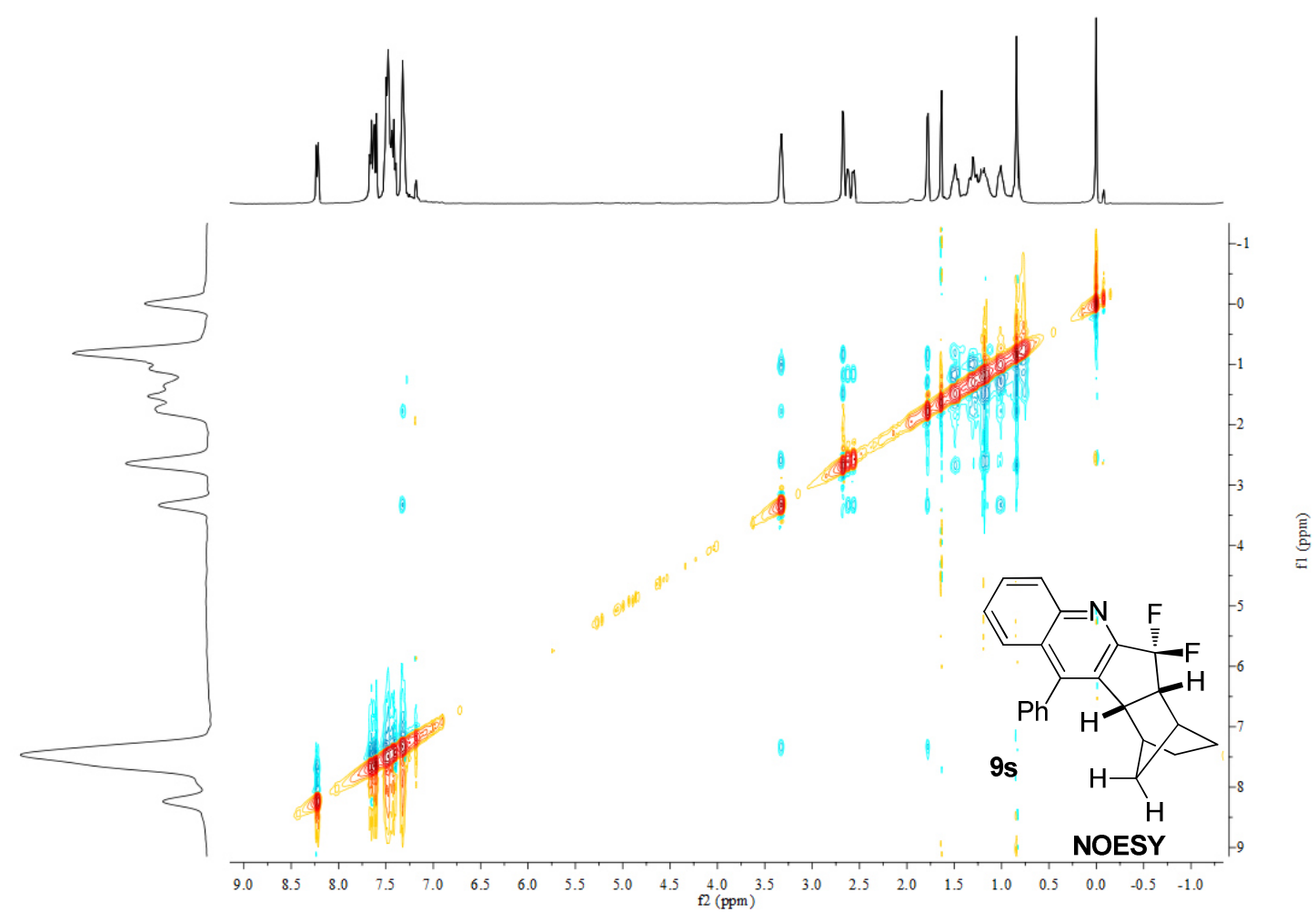

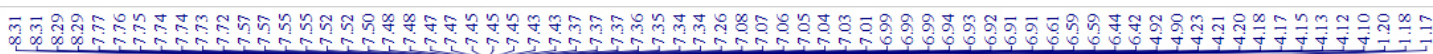

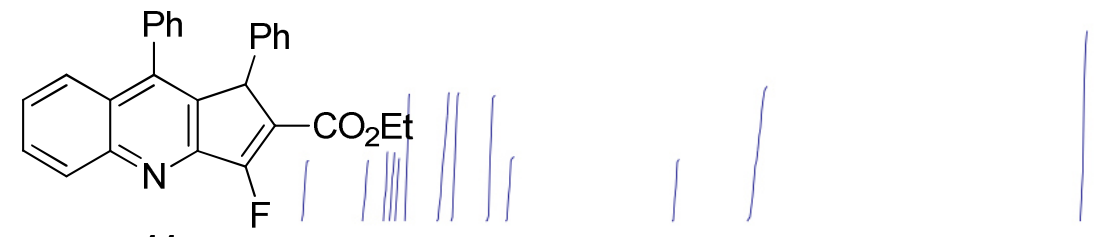

11

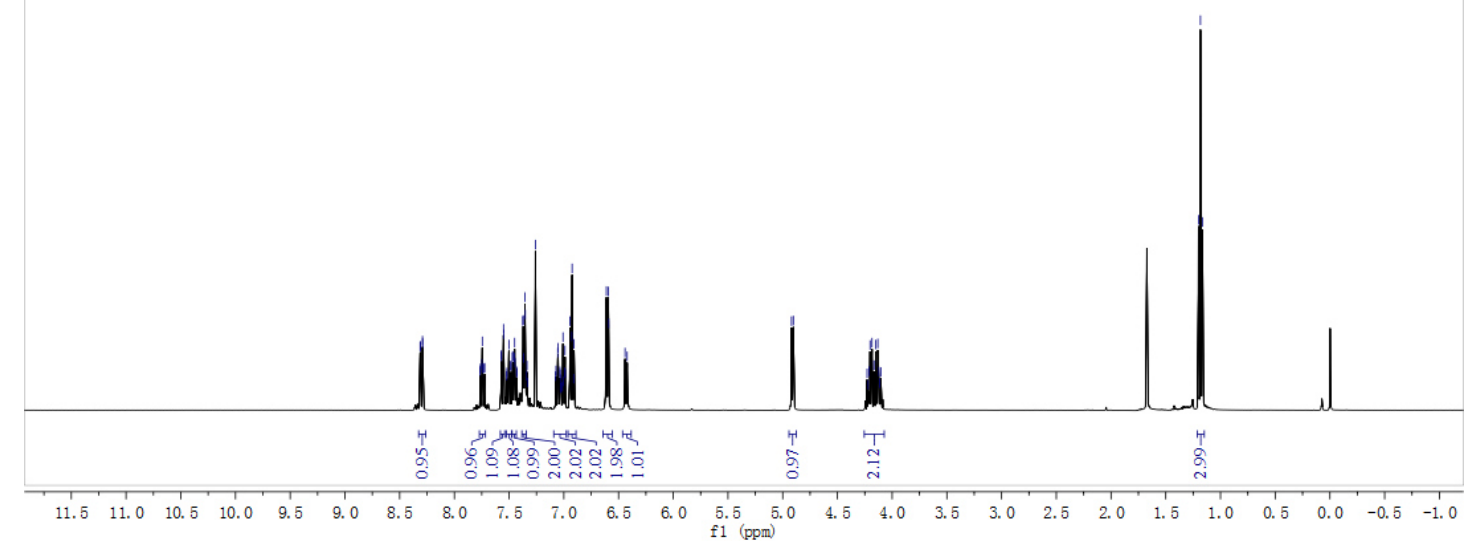




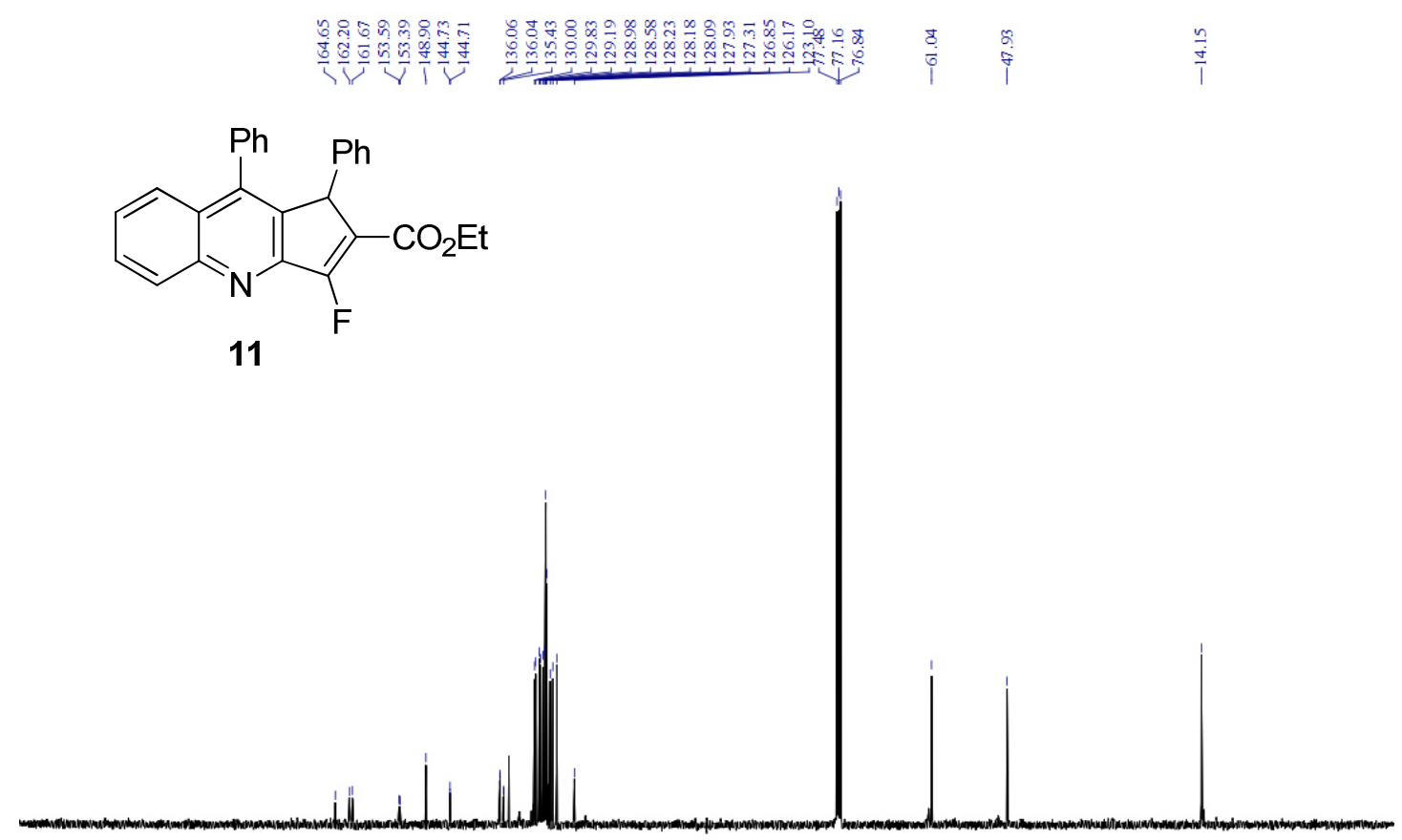

$\begin{array}{llllllllllllllllllllllllll}210 & 200 & 190 & 180 & 170 & 160 & 150 & 140 & 130 & 120 & 110 & 100 & 90 & 80 & 70 & 60 & 50 & 40 & 30 & 20 & 10 & 0 & -10\end{array}$

$\stackrel{8}{\stackrel{8}{7}}$<smiles>CCOC(=O)C1=C(F)c2nc3ccccc3c(-c3ccccc3)c2C1c1ccccc1</smiles>

11

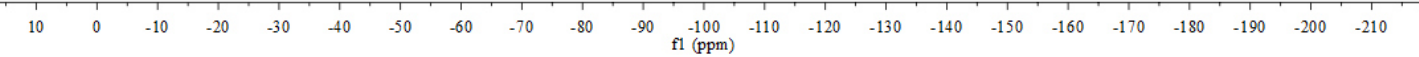


<smiles>CC1CC(C#N)CC(F)(F)c2c1nc1ccccc1c2P</smiles>

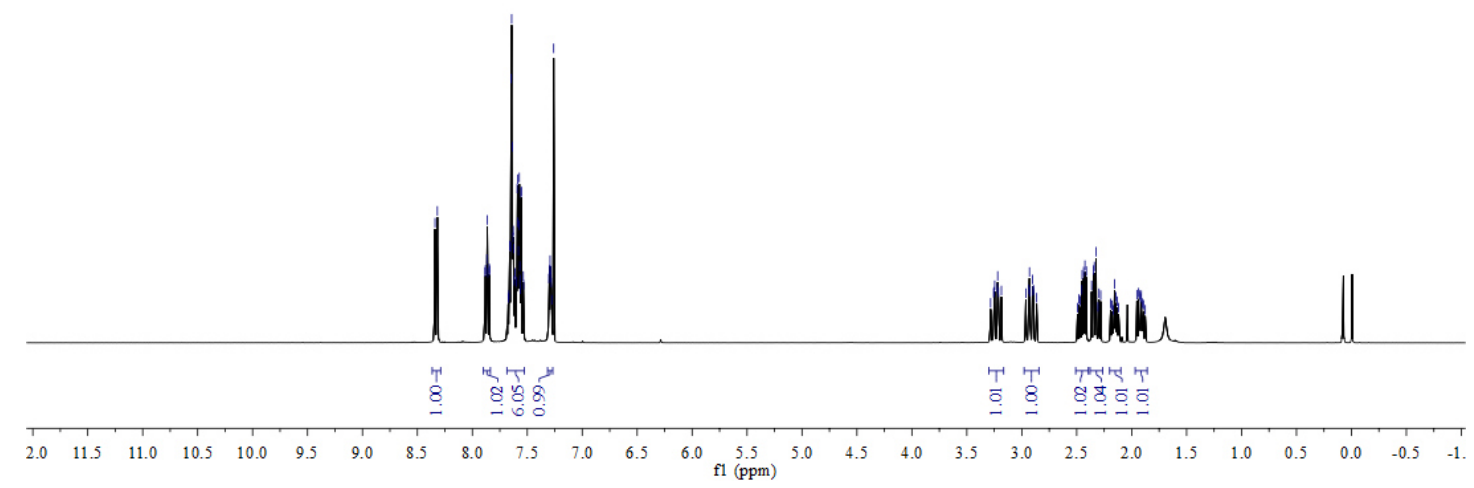

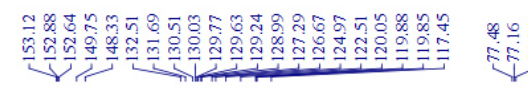

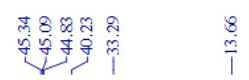

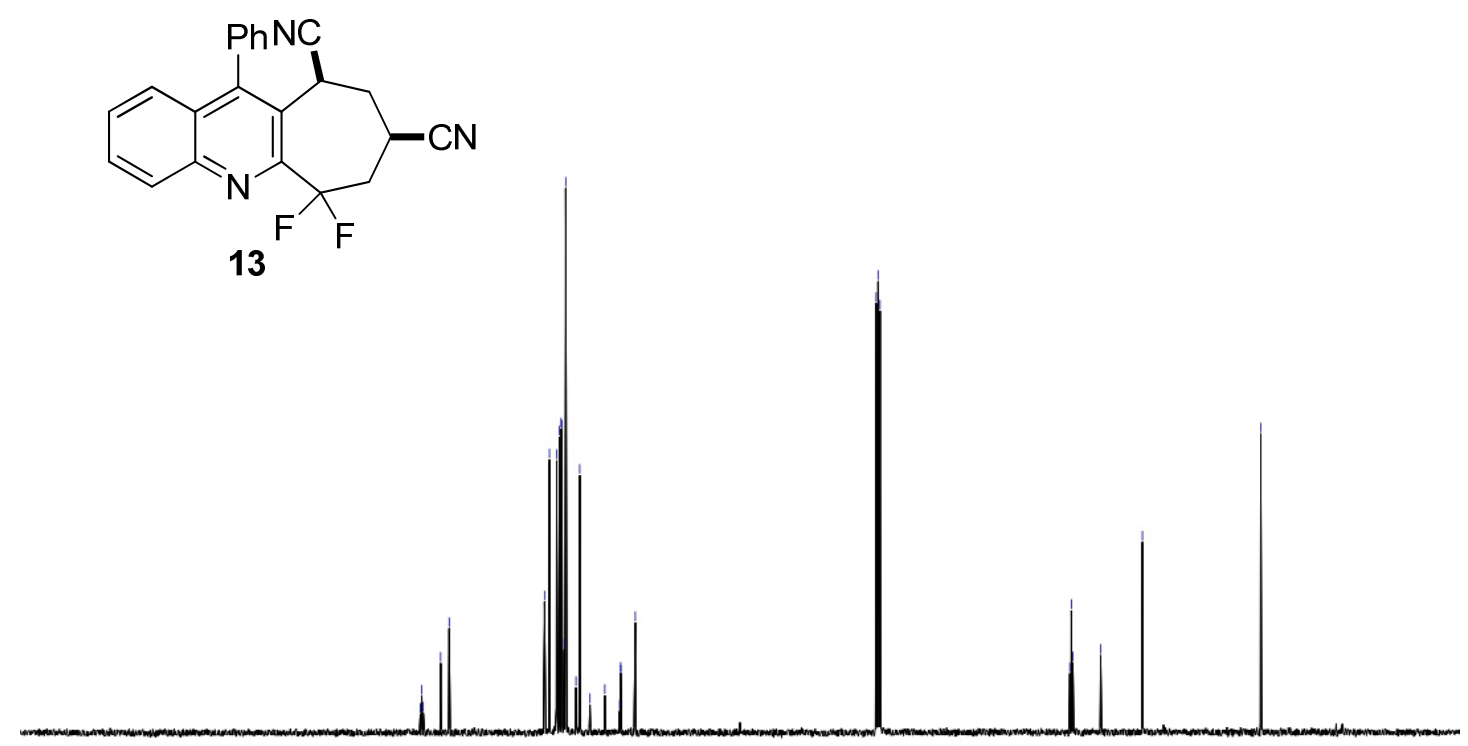

$\begin{array}{lllllllllllllllllllllllllll}1 & 10 & 210 & 190 & 180 & 170 & 160 & 150 & 140 & 130 & 120 & 110 & 100 & 90 & 80 & 70 & 60 & 50 & 40 & 30 & 20 & 10 & 0 & -10\end{array}$ 


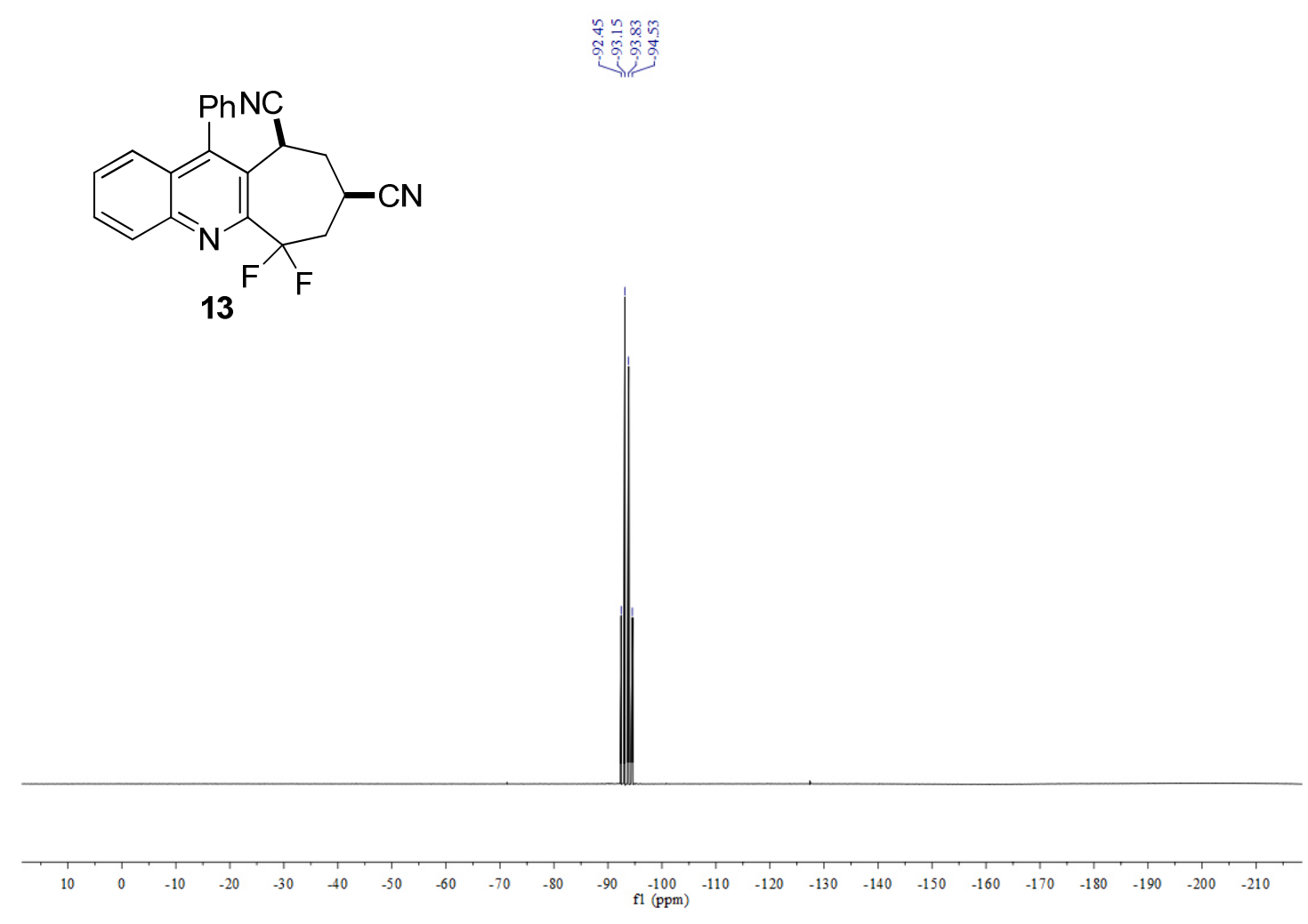

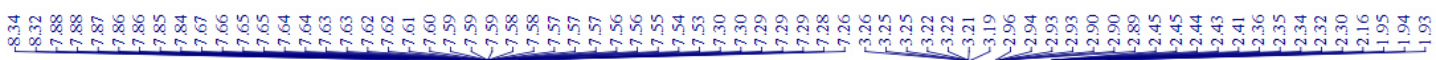
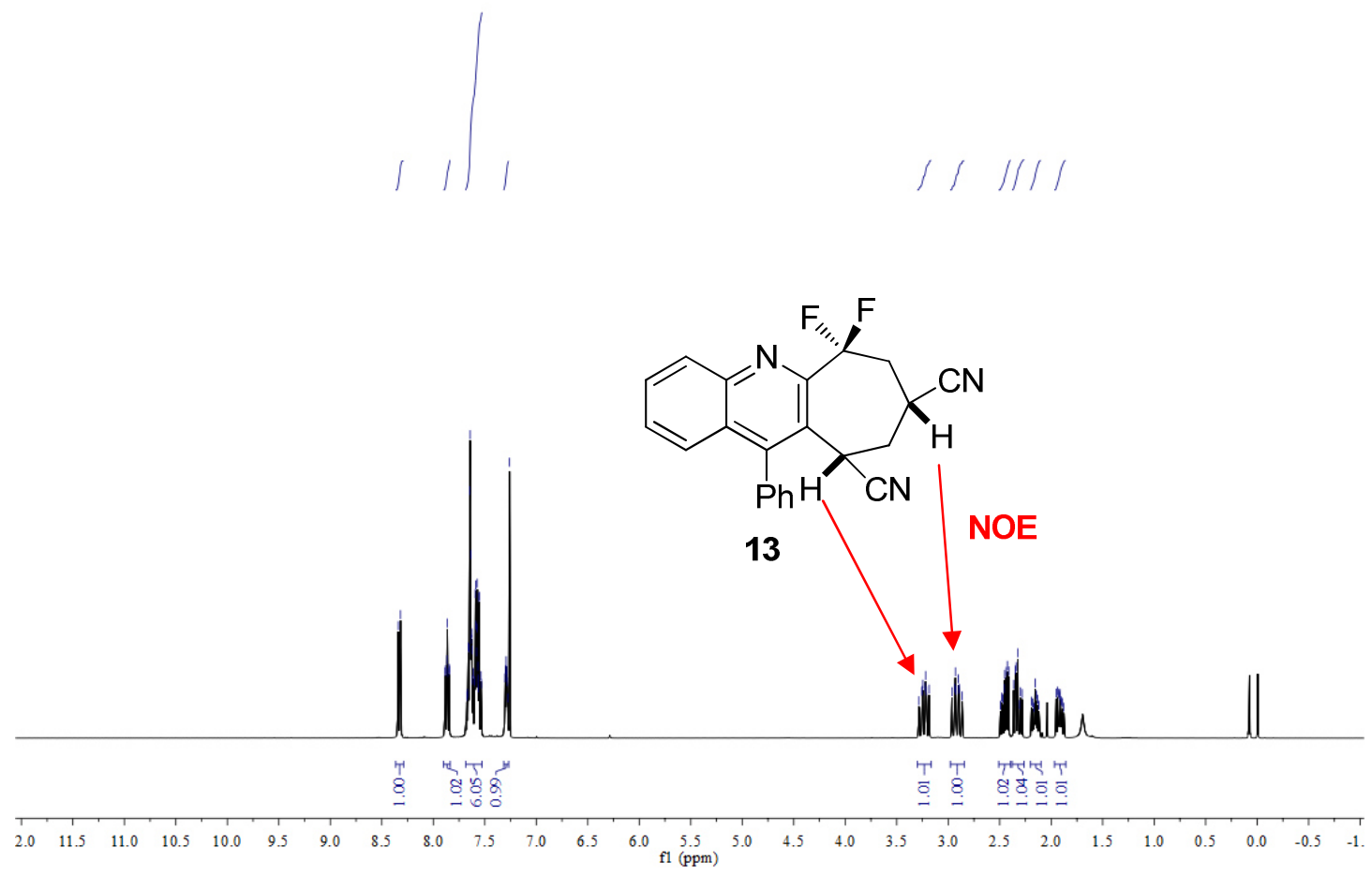


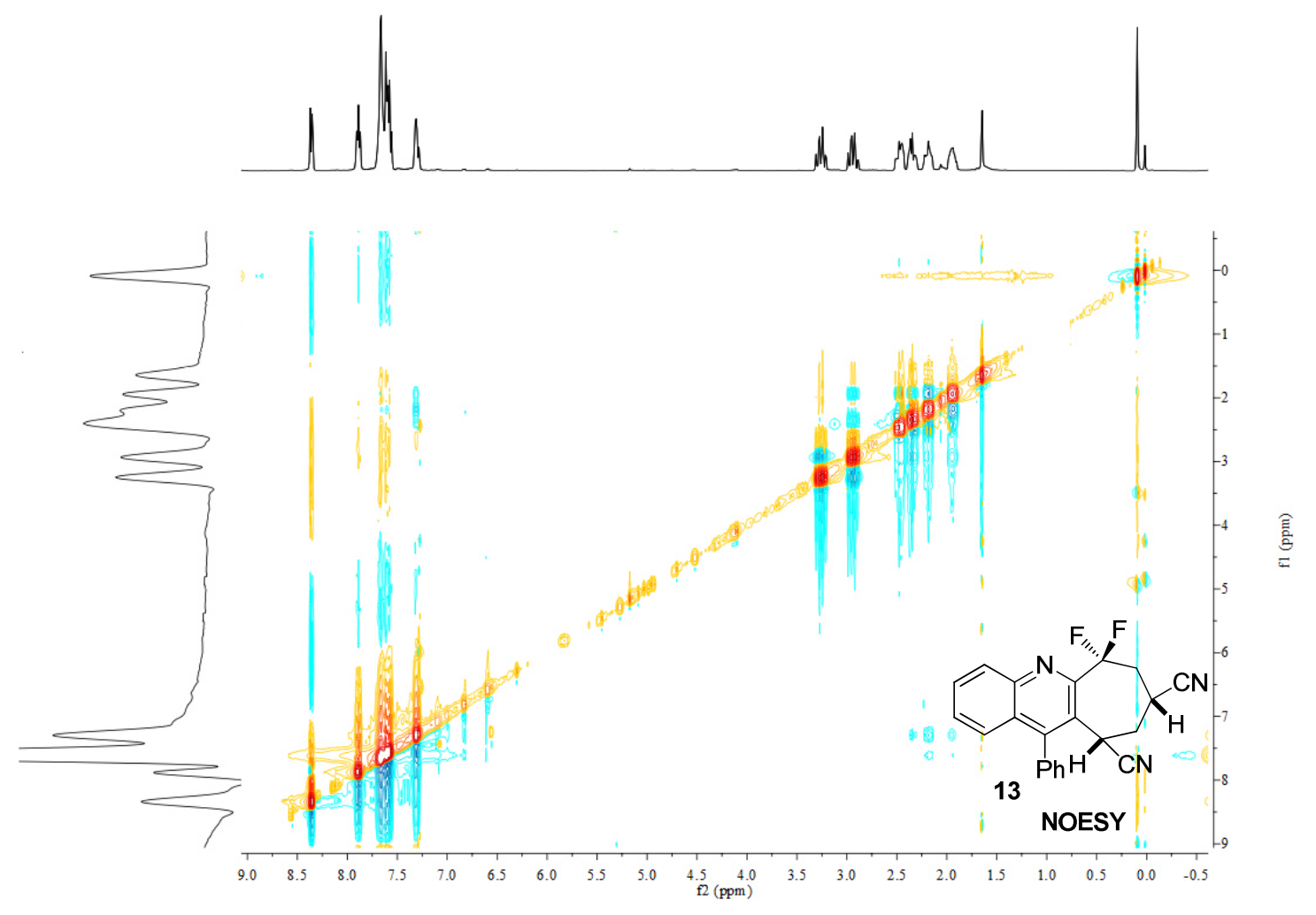

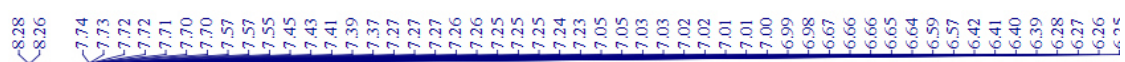

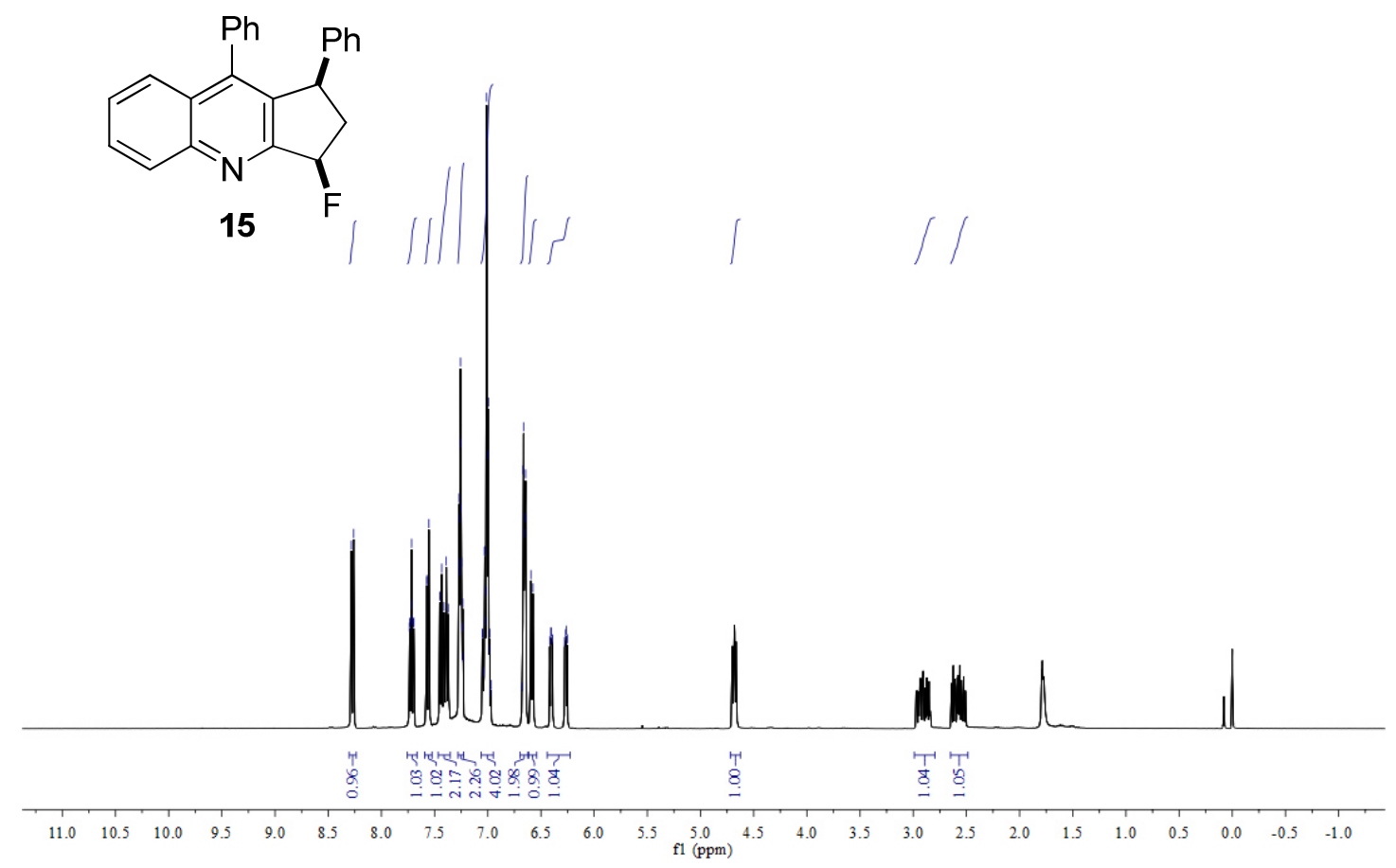


<smiles>FC1CC(c2ccccc2)c2c1nc1ccccc1c2-c1ccccc1</smiles>

15

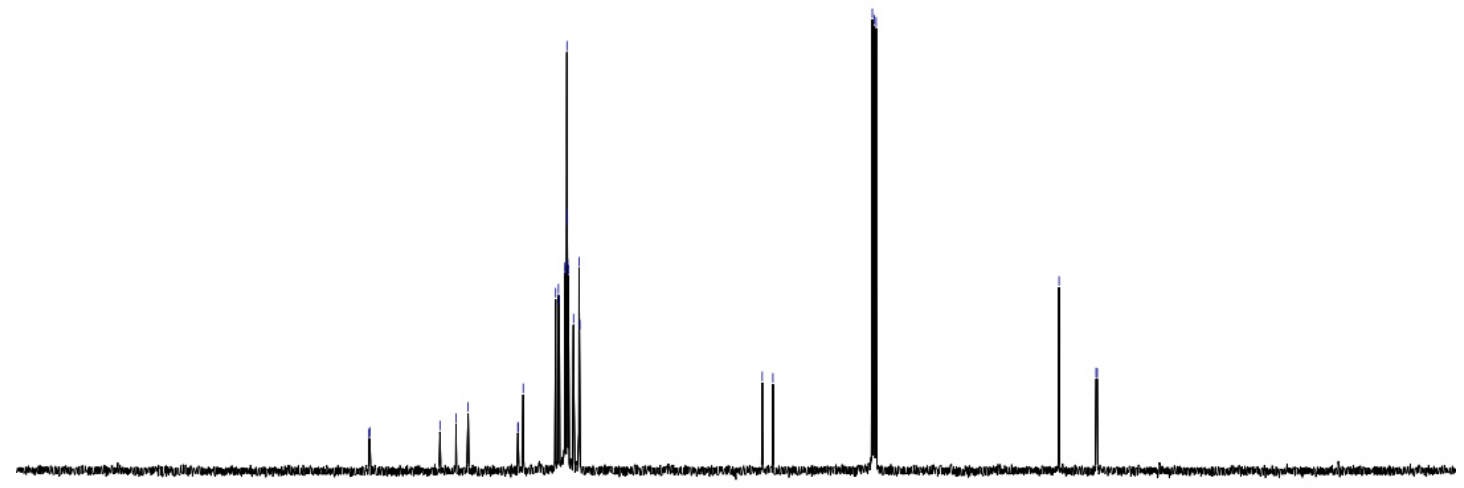

$\begin{array}{lllllllllllllllllllllllllll}1 & 110 & 200 & 190 & 180 & 170 & 160 & 150 & 140 & 130 & 120 & 110 & 100 & 10 & 80 & 70 & 60 & 50 & 40 & 30 & 20 & 10 & 0 & -10\end{array}$<smiles>FC1CC(c2ccccc2)c2c1nc1ccccc1c2-c1ccccc1</smiles> 

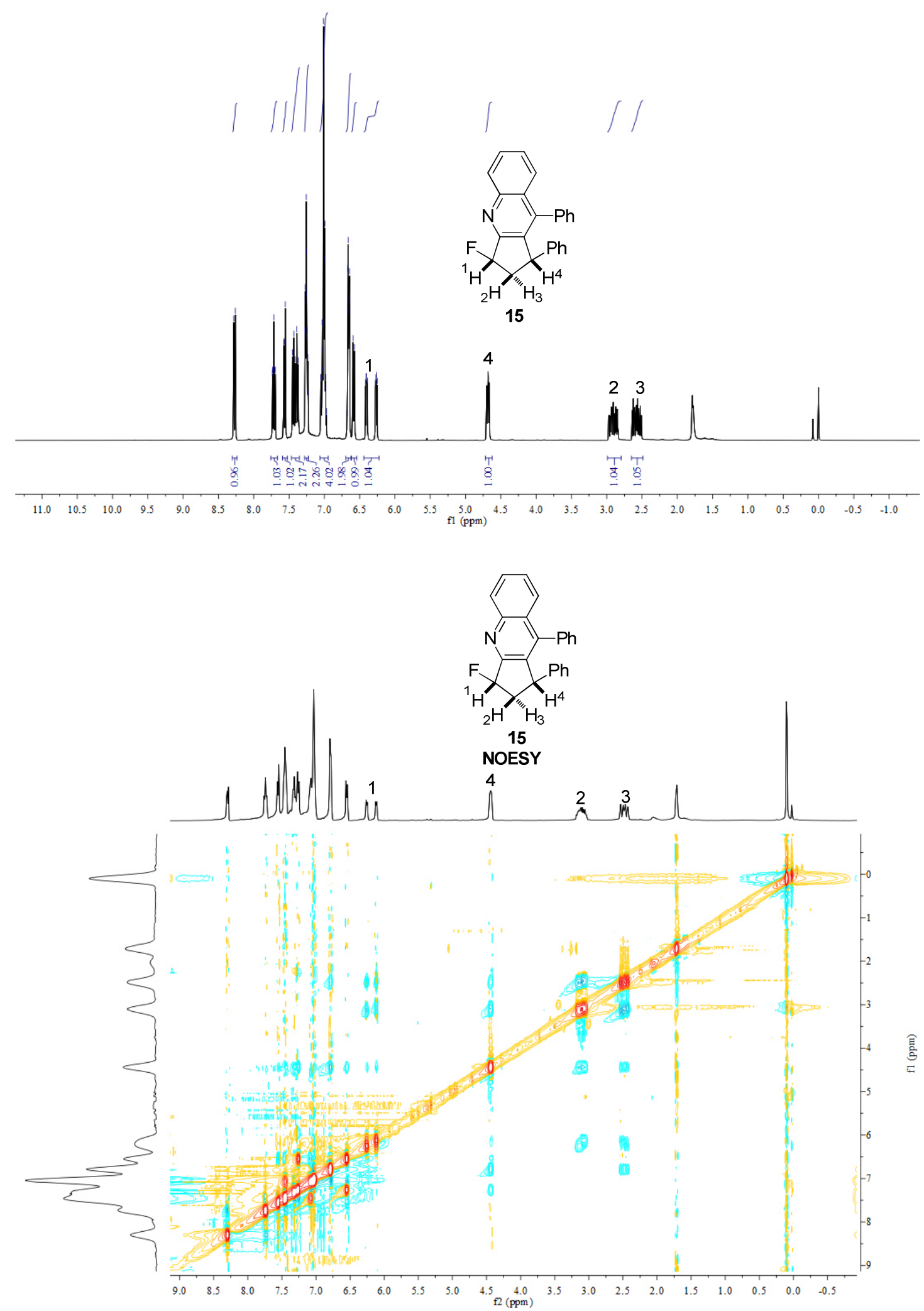

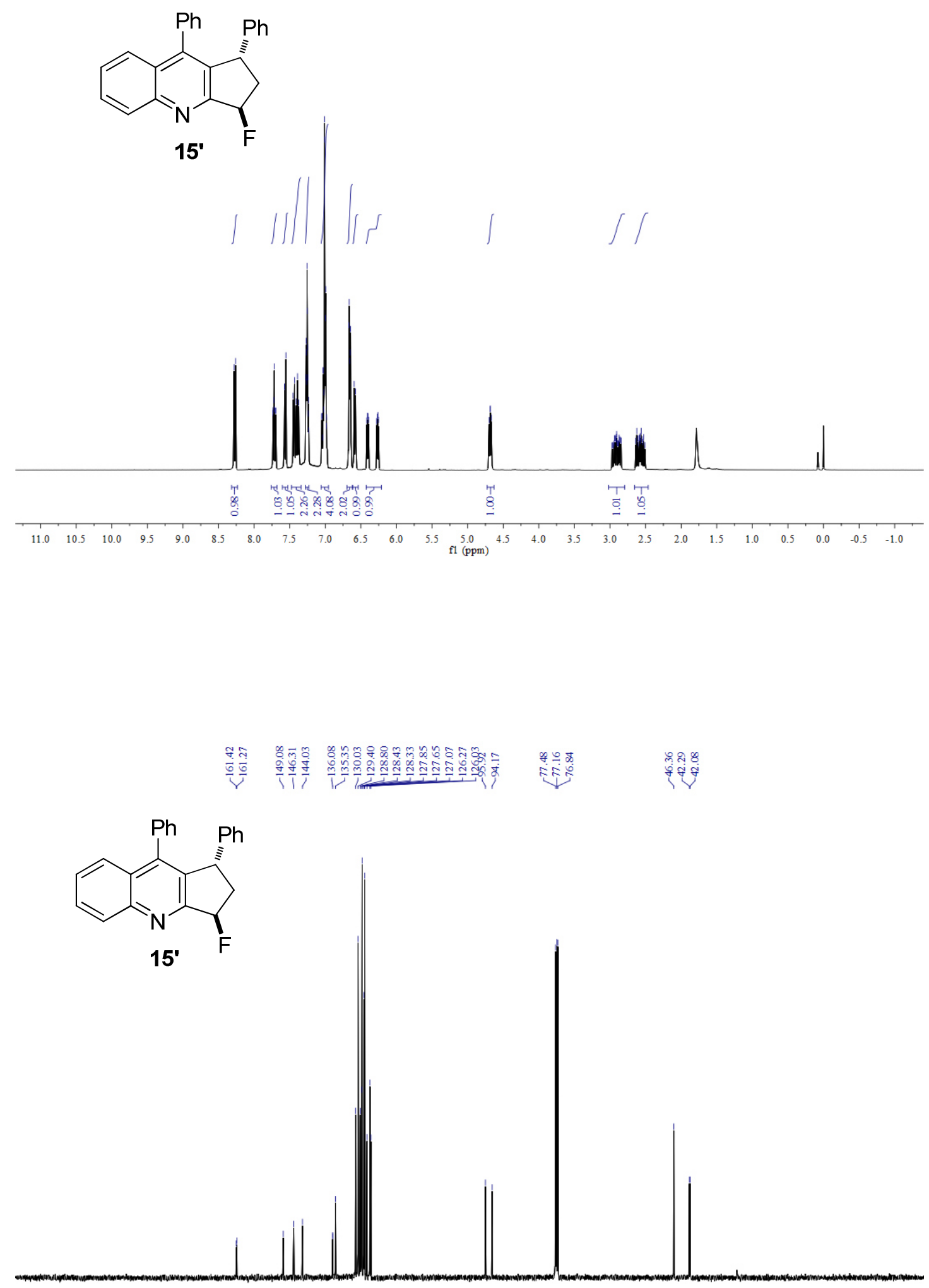
<smiles>FC1C[C@@H](c2ccccc2)c2c1nc1ccccc1c2-c1ccccc1</smiles>

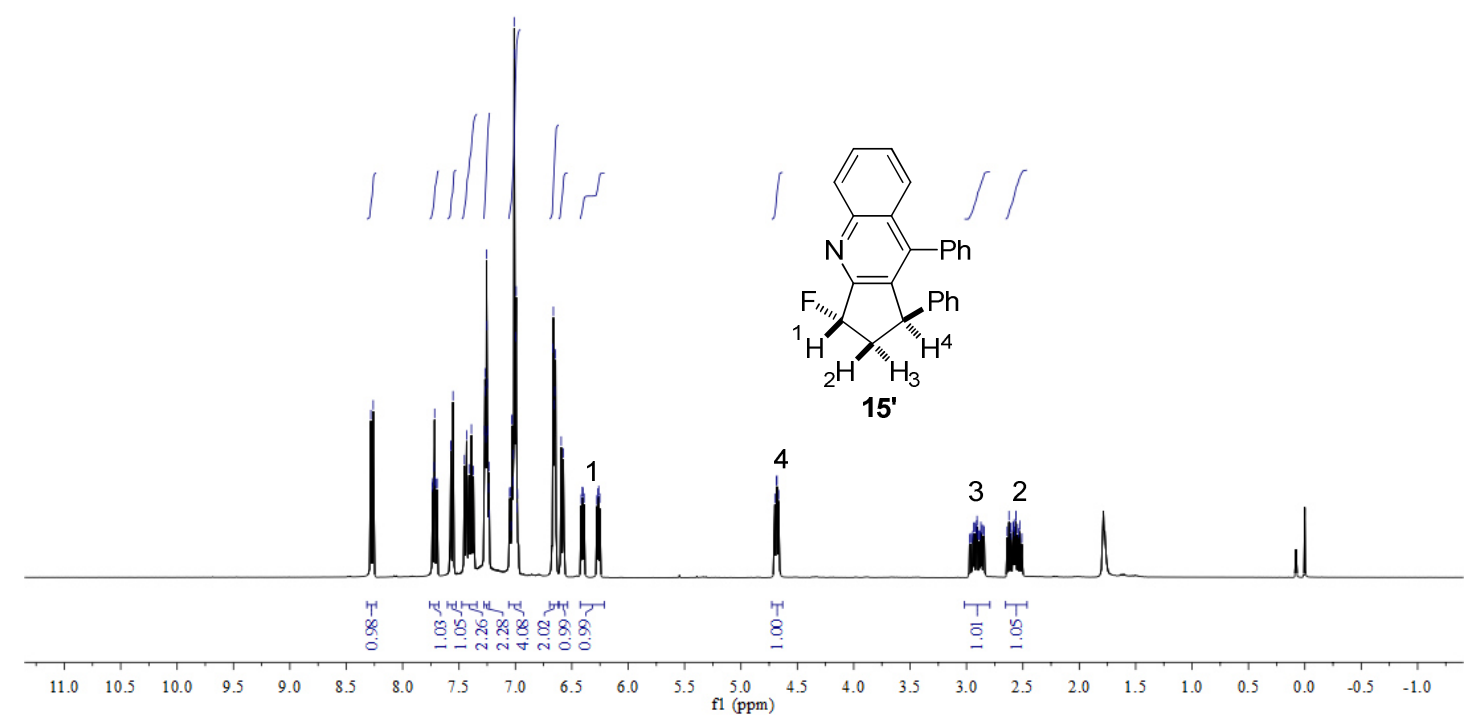




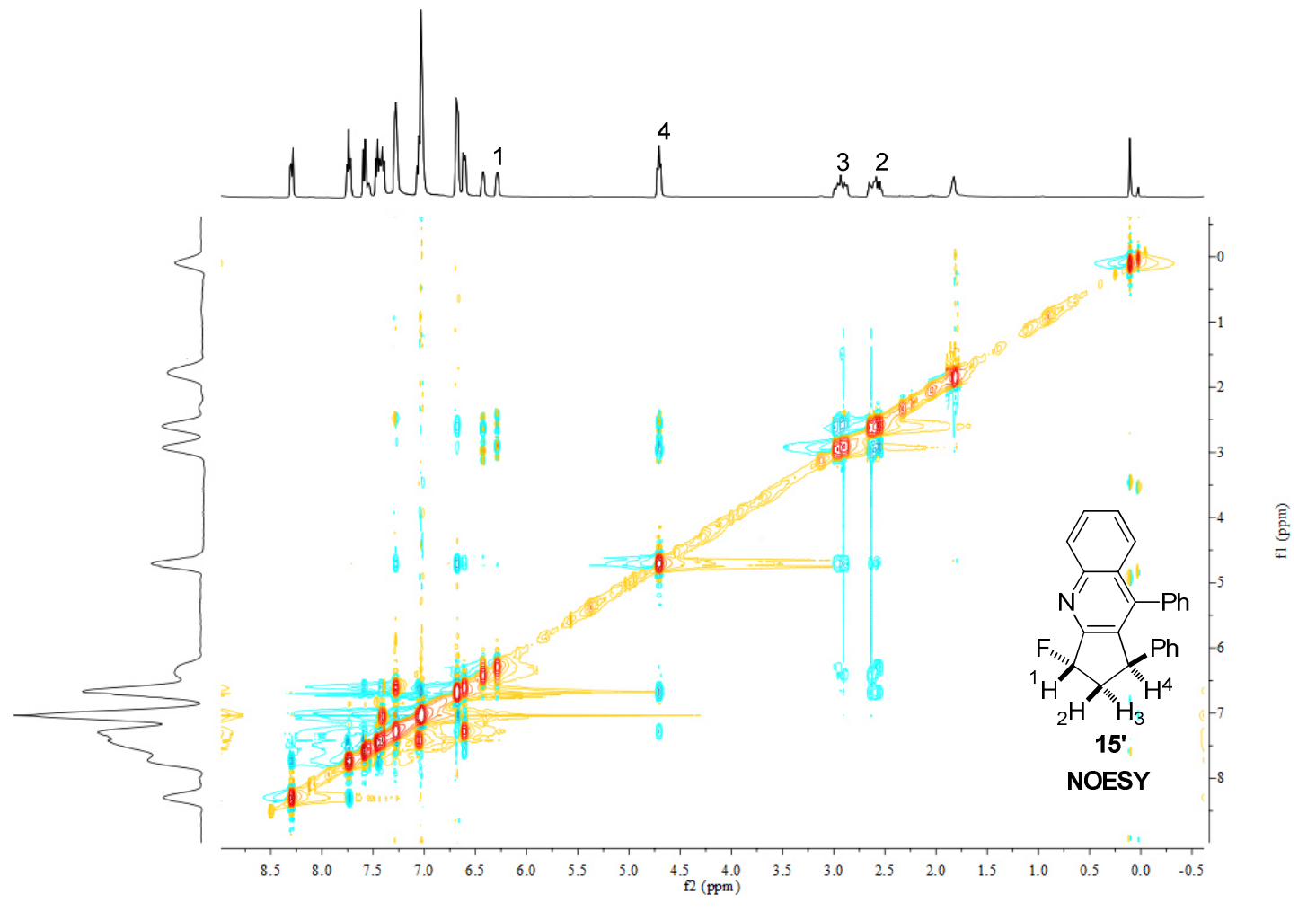

